\title{
Ligand-Enabled Ni-Al Bimetallic Catalysis for Nonchelated Dual C-H Annulation of Arylformamides and Alkynes
}

Yin-Xia Wang, Feng-Ping Zhang, Yu-Xin Luan, and Mengchun Ye*

State Key Laboratory and Institute of Elemento-Organic Chemistry, College of Chemistry, Nankai University, Tianjin 300071, China.

*Email: mcye@nankai.edu.cn

\section{Table of Contents}

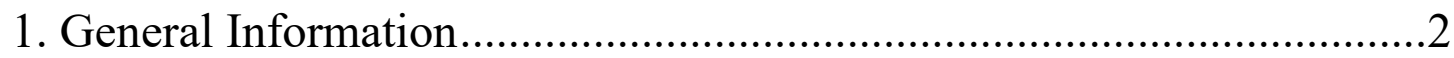

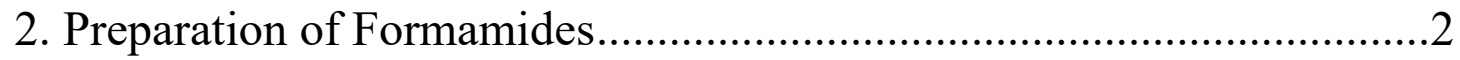

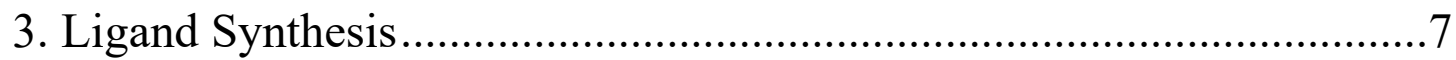

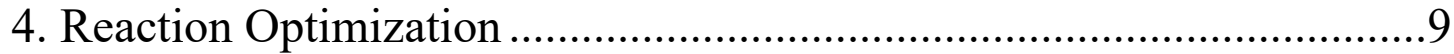

5. Typical Procedure for Ni-Catalyzed C-H Annulation..........................12

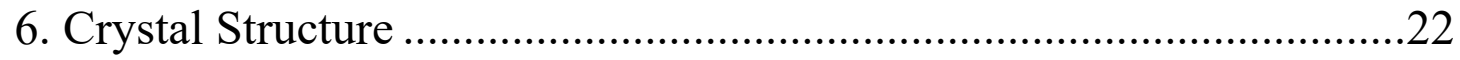

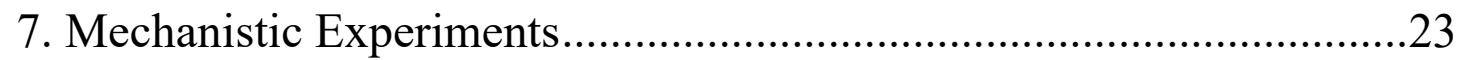

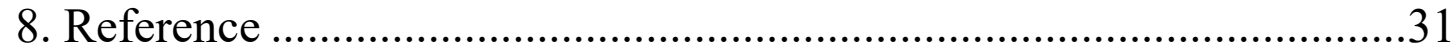

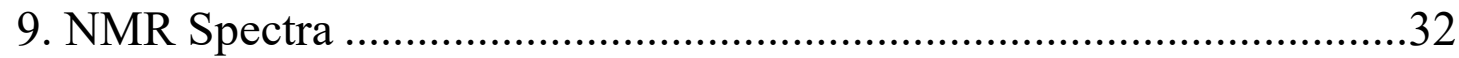




\section{General Information}

Unless stated otherwise, all glassware was flame-dried before use and all reactions were performed under an atmosphere of argon. All solvents were distilled from appropriate drying agents prior to use. All reagents were used as received from commercial suppliers unless otherwise stated. Non-commercially available substrates were synthesized following reported protocols. Melting points were measured on X-4B microscope melting point apparatus and uncorrected. Thin-layer chromatography (TLC) was performed by UV absorbance (254 nm). 200-300 mesh silica gel was used for column chromatography separation. NMR spectra were recorded on Bruker AV 400 spectrometer at $400 \mathrm{MHz}\left({ }^{1} \mathrm{H} \mathrm{NMR}\right), 100 \mathrm{MHz}\left({ }^{13} \mathrm{C} \mathrm{NMR}\right), 376 \mathrm{MHz}\left({ }^{19} \mathrm{~F} \mathrm{NMR}\right)$ and $162 \mathrm{MHz}\left({ }^{31} \mathrm{P} \mathrm{NMR}\right)$. Proton and carbon chemical shifts are reported relative to the solvent used as an internal reference $\left(\mathrm{CDCl}_{3}: \delta_{\mathrm{H}}=7.26 \mathrm{ppm} ; \delta_{\mathrm{C}}=77.16 \mathrm{ppm}\right)$. All coupling constants ( $J$ values) were reported in Hertz $(\mathrm{Hz})$. Multiplicities are reported as follows: singlet (s), doublet (d), doublet of doublets (dd), triplet (t), triplet of doublets $(\mathrm{td})$, quartet $(\mathrm{q})$, and multiplet $(\mathrm{m})$. High resolution mass spectra (HRMS) were recorded on an Agilent 6520 Q-TOF LC/MS with Electron Spray Ionization (ESI) resource. Single crystal X-ray diffraction data were collected on Rigaku Saturn70 diffractometer.

\section{Preparation of Arylformamides}

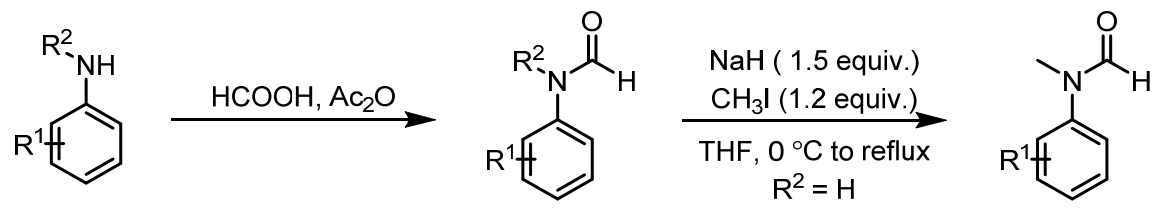

\section{$\mathbf{R}^{2} \neq \mathbf{H}:$}

A mixture of formic acid $(7.83 \mathrm{~g}, 0.17 \mathrm{~mol})$ and $\mathrm{Ac}_{2} \mathrm{O}(10.21 \mathrm{~g}, 0.1 \mathrm{~mol})$ was stirred at $70{ }^{\circ} \mathrm{C}$ for $1 \mathrm{~h}$ and then cooled to room temperature. Aniline $(0.02 \mathrm{~mol})$ was added slowly and the resulting mixture was stirred at $50{ }^{\circ} \mathrm{C}$. After the reaction was complete, the mixture was concentrated in vacuo, neutralized with saturated $\mathrm{NaHCO}_{3}$ and extracted with $\mathrm{CH}_{2} \mathrm{Cl}_{2}(3 \times 25 \mathrm{~mL})$. The combined organic layers were dried over anhydrous $\mathrm{Na}_{2} \mathrm{SO}_{4}$, filtered and concentrated in vacuo. The residue was purified by column chromatography on silica gel eluting with a gradient of EtOAc/ $n$-hexane.

$$
\mathbf{R}^{2}=\mathbf{H}:
$$

The crude formamide was dissolved in dry THF $(30 \mathrm{~mL})$ and cooled to $0{ }^{\circ} \mathrm{C}$. To this solution was added $\mathrm{NaH}$ (1.2 g, 1.5 equiv) portionwise and the mixture was allowed to warm to room temperature and stirred for 15 minutes before adding $\mathrm{CH}_{3} \mathrm{I}$ (3.41 g, 1.2 equiv) dropwise. The resulting mixture was heated to reflux in an oil bath overnight and then cooled to room temperature. The reaction was quenched with saturated $\mathrm{NH}_{4} \mathrm{Cl}$ $(10 \mathrm{~mL})$ and extracted with EtOAc $(3 \times 30 \mathrm{~mL})$. The combined organic layers were dried over anhydrous $\mathrm{Na}_{2} \mathrm{SO}_{4}$, filtered and concentrated in vacuo. The residue was purified by column chromatography on silica gel eluting with a gradient of EtOAc/n-hexane. 


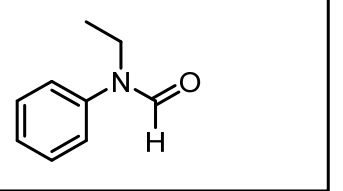

$N$-Ethyl- $N$-phenylformamide (1b) ${ }^{1}$

Colorless oil (2.8 g, 93\%). ${ }^{1} \mathbf{H}$ NMR $\left(400 \mathrm{MHz}, \mathrm{CDCl}_{3}\right) \delta 8.35(\mathrm{~s}, 1 \mathrm{H}), 7.44-7.38(\mathrm{~m}$, $2 \mathrm{H}), 7.33-7.27(\mathrm{~m}, 1 \mathrm{H}), 7.19-7.14(\mathrm{~m}, 2 \mathrm{H}), 3.86(\mathrm{q}, J=7.2 \mathrm{~Hz}, 2 \mathrm{H}), 1.16(\mathrm{t}, J=7.2$ $\mathrm{Hz}, 3 \mathrm{H}) .{ }^{13} \mathrm{C}$ NMR $\left(100 \mathrm{MHz}, \mathrm{CDCl}_{3}\right) \delta 162.0,140.8,129.7,129.3,126.9,126.1$, $124.3,40.1,13.1$.<smiles>O=CN(Cc1ccccc1)c1ccccc1</smiles>

$N$-Benzyl- $N$-phenylformamide (1c) ${ }^{1}$

Colorless oil (4.2 g, 99\%). ${ }^{1}$ H NMR (400 MHz, $\left.\mathrm{CDCl}_{3}\right) \delta 8.56(\mathrm{~s}, 1 \mathrm{H}), 7.36-7.32(\mathrm{~m}$, 2H), $7.29-7.20(\mathrm{~m}, 6 \mathrm{H}), 7.12-7.08(\mathrm{~m}, 2 \mathrm{H}), 5.00(\mathrm{~s}, 2 \mathrm{H}) .{ }^{13} \mathrm{C}$ NMR (100 MHz, $\left.\mathrm{CDCl}_{3}\right) \delta 162.5,141.1,136.7,129.6,128.7,127.9,127.5,127.0,124.1,48.9$.

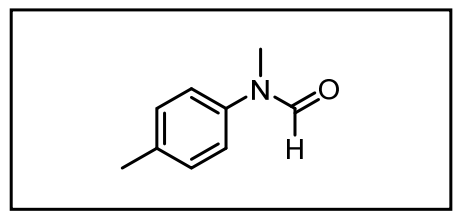

$\mathrm{N}$-Methyl- $\mathrm{N}$-(p-tolyl)formamide (1e) ${ }^{1}$

Colorless oil (2.8 g, 95\%). ${ }^{\mathbf{1}} \mathbf{H}$ NMR (400 MHz, $\left.\mathrm{CDCl}_{3}\right) \delta 8.42(\mathrm{~s}, 1 \mathrm{H}), 7.21$ (d, $J=7.6$ $\mathrm{Hz}, 2 \mathrm{H}), 7.06(\mathrm{~d}, J=7.6 \mathrm{~Hz}, 2 \mathrm{H}), 3.29(\mathrm{~s}, 3 \mathrm{H}), 2.36(\mathrm{~s}, 3 \mathrm{H}) .{ }^{13} \mathbf{C}$ NMR (100 MHz, $\left.\mathrm{CDCl}_{3}\right) \delta 162.4,139.7,136.4,130.2,122.6,32.2,20.9$.<smiles>CN(C=O)c1ccc(Br)cc1</smiles>

$\mathrm{N}$-(4-Isopropylphenyl)- $\mathrm{N}$-methylformamide (1f)

Colorless oil (2.7 g, 75\%). ${ }^{1} \mathbf{H}$ NMR (400 MHz, $\left.\mathrm{CDCl}_{3}\right) \delta 8.44(\mathrm{~s}, 1 \mathrm{H}), 7.27$ (d, $J=8.4$ $\mathrm{Hz}, 2 \mathrm{H}), 7.10(\mathrm{~d}, J=8.4 \mathrm{~Hz}, 2 \mathrm{H}), 3.31(\mathrm{~s}, 3 \mathrm{H}), 3.00-2.87(\mathrm{~m}, 1 \mathrm{H}), 1.26(\mathrm{~d}, J=6.8 \mathrm{~Hz}$, $6 \mathrm{H}) .{ }^{13} \mathrm{C}$ NMR $\left(100 \mathrm{MHz}, \mathrm{CDCl}_{3}\right) \delta 162.5,147.4,140.0,127.6,122.7,33.7,32.3,24.0$. HRMS (ESI) m/z: [M+H] $]^{+}$Calcd. for $\mathrm{C}_{11} \mathrm{H}_{16} \mathrm{NO}$ 178.1226; Found 178.1230.<smiles>CN(C=O)c1ccc(Br)cc1</smiles>

$\mathrm{N}$-(4-(tert-Butyl)phenyl)- $\mathrm{N}$-methylformamide (1g) ${ }^{2}$

White solid (3.5 g, 92\%), m.p. 48-50 ${ }^{\circ} \mathrm{C} .{ }^{1} \mathbf{H}$ NMR (400 MHz, $\left.\mathrm{CDCl}_{3}\right) \delta 8.45(\mathrm{~s}, 1 \mathrm{H})$, $7.42(\mathrm{~d}, J=8.4 \mathrm{~Hz}, 2 \mathrm{H}), 7.10(\mathrm{~d}, J=8.4 \mathrm{~Hz}, 2 \mathrm{H}), 3.30(\mathrm{~s}, 3 \mathrm{H}), 1.33(\mathrm{~s}, 9 \mathrm{H}) .{ }^{13} \mathbf{C}$ NMR $\left(100 \mathrm{MHz}, \mathrm{CDCl}_{3}\right) \delta 162.5,150.8,142.3,129.6,124.6,120.7,120.0,34.2,32.2,24.0$. 
<smiles>CN(C=O)c1ccc(Br)cc1</smiles>

$N$-(4-(Benzyloxy)phenyl)- $N$-methylformamide (1h)

White solid (4.4 g, 92\%), m.p. 92-94 ${ }^{\circ} \mathrm{C} .{ }^{1} \mathbf{H}$ NMR (400 MHz, $\left.\mathrm{CDCl}_{3}\right) \delta 8.35(\mathrm{~s}, 1 \mathrm{H})$, $7.46-7.33(\mathrm{~m}, 5 \mathrm{H}), 7.09(\mathrm{~d}, J=8.8 \mathrm{~Hz}, 2 \mathrm{H}), 7.00(\mathrm{~d}, J=8.8 \mathrm{~Hz}, 2 \mathrm{H}), 5.08(\mathrm{~s}, 2 \mathrm{H}), 3.27$ (s, 3H). ${ }^{13}$ C NMR (100 MHz, $\left.\mathrm{CDCl}_{3}\right) \delta 162.4,157.4,136.6,135.5,128.7,127.5,124.6$, 115.7, 70.3, 32.6. HRMS (ESI) m/z: $[\mathrm{M}+\mathrm{H}]^{+}$Calcd. for $\mathrm{C}_{15} \mathrm{H}_{16} \mathrm{NO}_{2} 242.1176$; Found 242.1176 .<smiles>CN(C=O)c1ccc(OC(F)(F)F)cc1</smiles>

$\mathbf{N}$-Methyl- $\boldsymbol{N}$-(4-(trifluoromethoxy)phenyl)formamide (1i) ${ }^{2}$

Colorless oil (3.9 g, 90\%). ${ }^{1} \mathbf{H}$ NMR $\left(400 \mathrm{MHz}, \mathrm{CDCl}_{3}\right) \delta 8.47(\mathrm{~s}, 1 \mathrm{H}), 7.28(\mathrm{~d}, J=9.2$ $\mathrm{Hz}, 2 \mathrm{H}), 7.21(\mathrm{~d}, J=9.2,2 \mathrm{H}), 3.32(\mathrm{~s}, 3 \mathrm{H}) .{ }^{13} \mathbf{C}$ NMR $\left(100 \mathrm{MHz}, \mathrm{CDCl}_{3}\right) \delta 162.0$, $147.1(\mathrm{q}, J=2.0 \mathrm{~Hz}), 140.8,123.5,122.3,120.3(\mathrm{q}, J=256.0 \mathrm{~Hz}), 32.0 .{ }^{19} \mathbf{F}$ NMR $(376$ $\left.\mathrm{MHz}, \mathrm{CDCl}_{3}\right) \delta-58.0$.<smiles>CN(C)c1ccc(N(C)C=O)cc1</smiles>

$\mathrm{N}$-Methyl- $\mathrm{N}$-(4-(methylamino)phenyl)formamide (1j)

Brown solid (2.6 g, 80\%), m.p. 80-82 ${ }^{\circ} \mathrm{C}^{1}{ }^{1} \mathbf{H}$ NMR $\left(400 \mathrm{MHz}, \mathrm{CDCl}_{3}\right) \delta 8.29(\mathrm{~s}, 1 \mathrm{H})$, $6.99(\mathrm{~d}, J=8.8 \mathrm{~Hz}, 2 \mathrm{H}), 6.60(\mathrm{~d}, J=8.8 \mathrm{~Hz}, 2 \mathrm{H}), 3.88(\mathrm{~s}, 1 \mathrm{H}), 3.24(\mathrm{~s}, 3 \mathrm{H}), 2.84(\mathrm{~s}, 3 \mathrm{H})$ ${ }^{13} \mathbf{C}$ NMR $\left(100 \mathrm{MHz}, \mathrm{CDCl}_{3}\right) \delta 162.6,148.4,131.9,125.0,112.7,32.9,30.7$. HRMS (ESI) m/z: [M+H] $]^{+}$Calcd. for $\mathrm{C}_{9} \mathrm{H}_{13} \mathrm{~N}_{2} \mathrm{O}$ 165.1022; Found 165.1023.<smiles>CN(C=O)c1ccc([N+](=O)[O-])cc1</smiles>

$\boldsymbol{N}$-(4-(Dimethylamino)phenyl)- $\boldsymbol{N}$-methylformamide $(\mathbf{1 k})^{3}$

Brown solid (2.8 g, 80\%), m.p. 99-101 ${ }^{\circ} \mathrm{C} .{ }^{1} \mathbf{H}$ NMR (400 MHz, $\left.\mathrm{CDCl}_{3}\right) \delta 8.31(\mathrm{~s}, 1 \mathrm{H})$, $7.05(\mathrm{~d}, J=8.8 \mathrm{~Hz}, 2 \mathrm{H}), 6.72(\mathrm{~d}, J=8.8 \mathrm{~Hz}, 2 \mathrm{H}), 3.25(\mathrm{~s}, 3 \mathrm{H}), 2.97(\mathrm{~s}, 6 \mathrm{H}) .{ }^{13} \mathbf{C}$ NMR $\left(100 \mathrm{MHz}, \mathrm{CDCl}_{3}\right) \delta 162.7,149.5,131.5,124.8,112.9,40.7,32.9$.<smiles>CN(C=O)c1ccc(-c2ccccc2)cc1</smiles>

$N$-([1,1'-Biphenyl]-4-yl)- $N$-methylformamide (1I) 
White solid (3.8 g, 90\%), m.p. 95-97 ${ }^{\circ} \mathrm{C} .{ }^{1} \mathbf{H}$ NMR (400 MHz, $\left.\mathrm{CDCl}_{3}\right) \delta 8.55(\mathrm{~s}, 1 \mathrm{H})$, $7.63(\mathrm{~d}, J=8.8 \mathrm{~Hz}, 2 \mathrm{H}), 7.58(\mathrm{~d}, J=7.2 \mathrm{~Hz}, 2 \mathrm{H}), 7.46(\mathrm{t}, J=7.6 \mathrm{~Hz}, 2 \mathrm{H}), 7.37(\mathrm{t}, J=7.4$ $\mathrm{Hz}, 1 \mathrm{H}), 7.25(\mathrm{~d}, J=7.6 \mathrm{~Hz}, 2 \mathrm{H}), 3.36(\mathrm{~s}, 3 \mathrm{H}) .{ }^{13} \mathbf{C} \mathbf{N M R}\left(100 \mathrm{MHz}, \mathrm{CDCl}_{3}\right) \delta 162.2$, 141.4, 139.9, 139.3, 129.0, 128.3, 127.6, 127.0, 122.5, 32.0. HRMS (ESI) m/z: $[\mathrm{M}+\mathrm{H}]^{+}$Calcd. for $\mathrm{C}_{14} \mathrm{H}_{14} \mathrm{NO} 212.1070$; Found 212.1072.<smiles>CN(C=O)c1ccc(F)cc1</smiles>

$N$-(4-Fluorophenyl)- $N$-methylformamide (1m) ${ }^{1}$

White solid (2.7 g, 88\%), m.p. 49-51 ${ }^{\circ} \mathrm{C} .{ }^{1} \mathbf{H}$ NMR (400 MHz, $\left.\mathrm{CDCl}_{3}\right) \delta 8.38(\mathrm{~s}, 1 \mathrm{H})$, $7.19-7.05(\mathrm{~m}, 4 \mathrm{H}), 3.29(\mathrm{~s}, 3 \mathrm{H}) .{ }^{13} \mathbf{C} \mathbf{N M R}\left(100 \mathrm{MHz}, \mathrm{CDCl}_{3}\right) \delta 161.01(\mathrm{~d}, J=244.8$ $\mathrm{Hz}), 162.1,138.3(\mathrm{~d}, J=2.8 \mathrm{~Hz}), 124.5(\mathrm{~d}, J=8.2 \mathrm{~Hz}), 116.4(\mathrm{~d}, J=22.6 \mathrm{~Hz}), 32.4 .{ }^{19} \mathbf{F}$ NMR $\left(376 \mathrm{MHz}, \mathrm{CDCl}_{3}\right) \delta-115.2$.

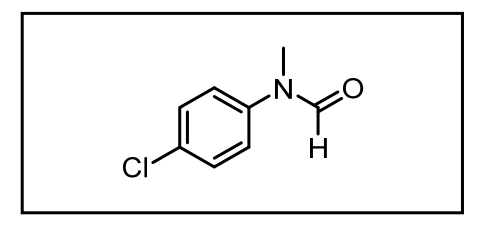

\section{$\mathrm{N}$-(4-Chlorophenyl)- $\mathrm{N}$-methylformamide (1n) ${ }^{1}$}

White solid (3.1 g, 92\%), m.p. 48-50 ${ }^{\circ} \mathrm{C} .{ }^{1} \mathbf{H}$ NMR (400 MHz, $\left.\mathrm{CDCl}_{3}\right) \delta 8.44(\mathrm{~s}, 1 \mathrm{H})$, $7.37(\mathrm{~d}, J=8.8 \mathrm{~Hz}, 2 \mathrm{H}), 7.11(\mathrm{~d}, J=8.8 \mathrm{~Hz}, 2 \mathrm{H}), 3.29$ (s, 3H). ${ }^{13} \mathbf{C}$ NMR (100 MHz, $\left.\mathrm{CDCl}_{3}\right) \delta 162.0,140.8,132.0,129.8,123.5,32.1$.

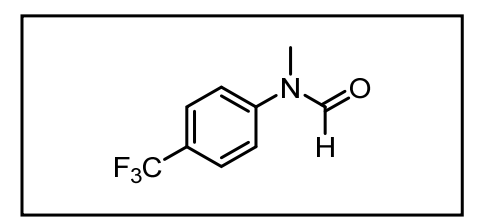

$\mathrm{N}$-Methyl- $\mathrm{N}$-(4-(trifluoromethyl)phenyl)formamide (10) ${ }^{1}$

White solid (1.8 g, 45\%), m.p. 36-38 ${ }^{\circ} \mathrm{C} .{ }^{1} \mathbf{H}$ NMR (400 MHz, $\left.\mathrm{CDCl}_{3}\right) \delta 8.60(\mathrm{~s}, 1 \mathrm{H})$, $7.68(\mathrm{~d}, J=8.4 \mathrm{~Hz}, 2 \mathrm{H}), 7.29(\mathrm{~d}, J=8.4 \mathrm{~Hz}, 2 \mathrm{H}), 3.36(\mathrm{~s}, 3 \mathrm{H}) .{ }^{13} \mathbf{C}$ NMR $(100 \mathrm{MHz}$, $\left.\mathrm{CDCl}_{3}\right) \delta 161.9,145.2,128.1(\mathrm{q}, J=32.8 \mathrm{~Hz}), 126.9(\mathrm{q}, J=3.8 \mathrm{~Hz}), 122.7(\mathrm{q}, J=270.2$ $\mathrm{Hz}), 121.5,31.6 .{ }^{19}$ F NMR $\left(376 \mathrm{MHz}, \mathrm{CDCl}_{3}\right) \delta-62.3$.

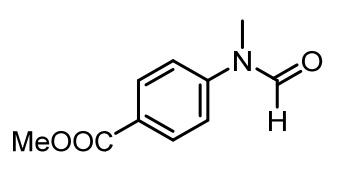

Methyl 4-( $N$-methylformamido)benzoate (1p)

White solid (1.5 g, 40\%), m.p. 87-89 ${ }^{\circ} \mathrm{C} .{ }^{1} \mathbf{H}$ NMR (400 MHz, $\left.\mathrm{CDCl}_{3}\right) \delta 8.64(\mathrm{~s}, 1 \mathrm{H})$, $8.09(\mathrm{~d}, J=8.0 \mathrm{~Hz}, 2 \mathrm{H}), 7.23(\mathrm{~d}, J=8.0 \mathrm{~Hz}, 2 \mathrm{H}), 3.93(\mathrm{~s}, 3 \mathrm{H}), 3.36(\mathrm{~s}, 3 \mathrm{H}) .{ }^{13} \mathbf{C ~ N M R}$ $\left(100 \mathrm{MHz}, \mathrm{CDCl}_{3}\right) \delta 166.3,162.0,146.1,131.3,127.6,120.7,52.4,31.6$. HRMS (ESI) $\mathrm{m} / \mathrm{z}:[\mathrm{M}+\mathrm{H}]^{+}$Calcd. for $\mathrm{C}_{10} \mathrm{H}_{12} \mathrm{NO}_{3}$ 194.0812; Found 194.0814. 


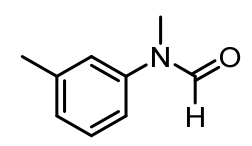

$\mathbf{N}$-Methyl- $\mathbf{N}$-(m-tolyl)formamide (1q) ${ }^{1}$

Colorless oil (2.7 g, 90\%). ${ }^{1} \mathbf{H}$ NMR $\left(400 \mathrm{MHz}, \mathrm{CDCl}_{3}\right) \delta 8.46(\mathrm{~s}, 1 \mathrm{H}), 7.32-7.26(\mathrm{~m}$, 1H), 7.09 (d, $J=7.2 \mathrm{~Hz}, 1 \mathrm{H}), 7.01-6.94(\mathrm{~m}, 2 \mathrm{H}), 3.31(\mathrm{~s}, 3 \mathrm{H}), 2.39(\mathrm{~s}, 3 \mathrm{H}) .{ }^{13} \mathbf{C}$ NMR $\left(100 \mathrm{MHz}, \mathrm{CDCl}_{3}\right) \delta 162.4,142.2,139.7,129.4,127.2,123.1,119.5,32.1,21.4$.<smiles>CC(C)c1cccc(N(C)C=O)c1</smiles>

\section{$\mathrm{N}$-(3-Isopropylphenyl)- $\mathrm{N}$-methylformamide (1r)}

Colorless oil (2.5 g, 70\%). ${ }^{1} \mathbf{H}$ NMR $\left(400 \mathrm{MHz}, \mathrm{CDCl}_{3}\right) \delta 8.47(\mathrm{~s}, 1 \mathrm{H}), 7.33$ (t, $J=7.8$ $\mathrm{Hz}, 1 \mathrm{H}), 7.15(\mathrm{~d}, J=8.0 \mathrm{~Hz}, 1 \mathrm{H}), 7.02-6.97(\mathrm{~m}, 2 \mathrm{H}), 3.32(\mathrm{~s}, 3 \mathrm{H}), 2.99-2.87(\mathrm{~m}, 1 \mathrm{H})$, $1.27(\mathrm{~d}, J=6.8 \mathrm{~Hz}, 6 \mathrm{H}) .{ }^{13} \mathbf{C}$ NMR $\left(100 \mathrm{MHz}, \mathrm{CDCl}_{3}\right) \delta 162.4,149.6,139.6,126.5$, 122.2, 34.6, 32.1, 31.4. HRMS (ESI) m/z: $[\mathrm{M}+\mathrm{H}]^{+}$Calcd. for $\mathrm{C}_{11} \mathrm{H}_{16} \mathrm{NO} 178.1226$; Found 178.1230.<smiles>CN(C=O)c1cccc(F)c1</smiles>

\section{$N$-(3-Fluorophenyl)- $N$-methylformamide (1s)}

Colorless oil (2.3 g, 75\%). ${ }^{1} \mathbf{H}$ NMR $\left(400 \mathrm{MHz}, \mathrm{CDCl}_{3}\right) \delta 8.53(\mathrm{~s}, 1 \mathrm{H}), 7.43-7.32(\mathrm{~m}$, $1 \mathrm{H}), 7.01-6.95(\mathrm{~m}, 2 \mathrm{H}), 6.92-6.87(\mathrm{~m}, 1 \mathrm{H}), 3.31(\mathrm{~s}, 3 \mathrm{H}) .{ }^{13} \mathbf{C}$ NMR $(100 \mathrm{MHz}$, $\left.\mathrm{CDCl}_{3}\right) \delta 164.5,162.0,143.8(\mathrm{~d}, J=9.6 \mathrm{~Hz}), 130.9(\mathrm{~d}, J=9.4 \mathrm{~Hz}), 117.4(\mathrm{~d}, J=3.0 \mathrm{~Hz})$, $113.1(\mathrm{~d}, J=21.0 \mathrm{~Hz}), 109.3(\mathrm{~d}, J=24.0 \mathrm{~Hz}), 31.9 .{ }^{19} \mathbf{F} \mathbf{N M R}\left(376 \mathrm{MHz}, \mathrm{CDCl}_{3}\right) \delta$ -110.4. HRMS (ESI) m/z: [M+H] $]^{+}$Calcd. for $\mathrm{C}_{8} \mathrm{H}_{9} \mathrm{FNO}$ 154.0663; Found 154.0666.

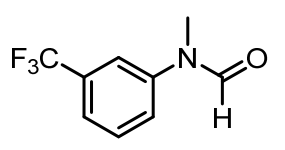

\section{$\mathrm{N}$-Methyl- $\mathrm{N}$-(3-(trifluoromethyl)phenyl)formamide (1t)}

Colorless oil (2.0 g, 50\%). ${ }^{1} \mathbf{H}$ NMR $\left(400 \mathrm{MHz}, \mathrm{CDCl}_{3}\right) \delta 8.53(\mathrm{~s}, 1 \mathrm{H}), 7.59-7.50(\mathrm{~m}$, 2H), $7.42(\mathrm{~s}, 1 \mathrm{H}), 7.39-7.35(\mathrm{~m}, 1 \mathrm{H}), 3.36(\mathrm{~s}, 3 \mathrm{H}) .{ }^{13} \mathbf{C} \mathbf{~ N M R}\left(100 \mathrm{MHz}, \mathrm{CDCl}_{3}\right) \delta$ $162.0,142.8,132.3(\mathrm{q}, J=32.6 \mathrm{~Hz}), 130.4,125.3,123.6(\mathrm{q}, J=271.8 \mathrm{~Hz}), 123.0(\mathrm{q}, J=$ $3.8 \mathrm{~Hz}), 118.8(\mathrm{q}, J=3.8 \mathrm{~Hz}), 32.0 .{ }^{19} \mathbf{F}$ NMR $\left(376 \mathrm{MHz}, \mathrm{CDCl}_{3}\right) \delta-62.8$. HRMS (ESI) $\mathrm{m} / \mathrm{z}$ : $[\mathrm{M}+\mathrm{H}]^{+}$Calcd. for $\mathrm{C}_{9} \mathrm{H}_{9} \mathrm{~F}_{3} \mathrm{NO} 204.0631$; Found 204.0628.

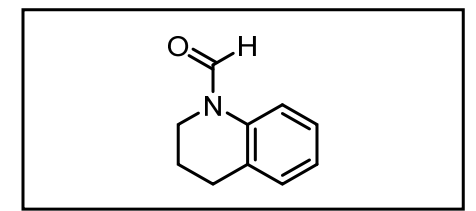




\section{3,4-Dihydroquinoline-1(2H)-carbaldehyde (1u) ${ }^{1}$}

Colorless oil (3.2 g, 99\%). ${ }^{1} \mathbf{H}$ NMR $\left(400 \mathrm{MHz}, \mathrm{CDCl}_{3}\right) \delta 8.79(\mathrm{~s}, 0.93 \mathrm{H}), 8.30(\mathrm{~s}$, $0.07 \mathrm{H}), 7.24-7.04(\mathrm{~m}, 4 \mathrm{H}), 3.81(\mathrm{t}, J=6.0 \mathrm{~Hz}, 1.97 \mathrm{H}), 3.67(\mathrm{t}, J=5.6 \mathrm{~Hz}, 0.18 \mathrm{H})$, $2.91(\mathrm{t}, \mathrm{J}=6.6 \mathrm{~Hz}, 0.18 \mathrm{H}), 2.82(\mathrm{t}, J=6.4 \mathrm{~Hz}, 1.98 \mathrm{H}), 2.06-1.99(\mathrm{~m}, 0.18 \mathrm{H}), 1.99-$ $1.93(\mathrm{~m}, 1.98 \mathrm{H}) ;{ }^{13} \mathbf{C}$ NMR $\left(100 \mathrm{MHz}, \mathrm{CDCl}_{3}\right)$ major isomer: $\delta 161.0,137.1,129.5$, $128.8,127.0,124.4,116.9,40.2,27.0,22.2$; minor isomer: $\delta$ 161.4, 134.1, 130.2, $129.2,127.5,126.1,122.2,46.1,26.9,23.0$.

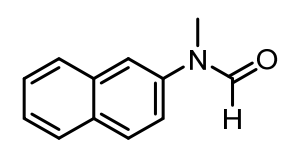

$\mathrm{N}$-Methyl- $\boldsymbol{N}$-(naphthalen-2-yl)formamide (1v) ${ }^{2}$

Greyish solid (2.8 g, 75\%), m.p. 88-90 ${ }^{\circ} \mathrm{C} .{ }^{1} \mathbf{H}$ NMR (400 MHz, $\left.\mathrm{CDCl}_{3}\right) \delta 88.61(\mathrm{~s}, 1 \mathrm{H})$, $7.90(\mathrm{~d}, J=8.8 \mathrm{~Hz}, 1 \mathrm{H}), 7.84(\mathrm{t}, J=9.0 \mathrm{~Hz}, 2 \mathrm{H}), 7.59-7.47(\mathrm{~m}, 3 \mathrm{H}), 7.37-7.31(\mathrm{~m}$, $1 \mathrm{H}), 3.42$ (s, 3H). ${ }^{13} \mathrm{C}$ NMR $\left(100 \mathrm{MHz}, \mathrm{CDCl}_{3}\right) \delta 162.6,139.6,133.6,131.7,129.8$, $127.8,127.7,127.2,126.2,121.2,120.0,32.2$.

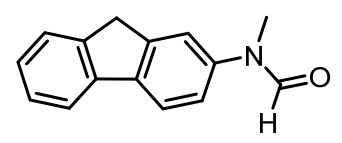

$\mathrm{N}$-(9H-Fluoren-2-yl)- $\mathrm{N}$-methylformamide (1w)

Greyish solid (2.7 g, 60\%), m.p. $108-109{ }^{\circ} \mathrm{C} .{ }^{1} \mathbf{H}$ NMR $\left(400 \mathrm{MHz}, \mathrm{CDCl}_{3}\right) \delta 8.44(\mathrm{~s}$, $1 \mathrm{H}), 7.68(\mathrm{~d}, J=8.0 \mathrm{~Hz}, 2 \mathrm{H}), 7.46(\mathrm{~d}, J=7.6 \mathrm{~Hz}, 1 \mathrm{H}), 7.31(\mathrm{t}, J=7.4 \mathrm{~Hz}, 1 \mathrm{H}), 7.24(\mathrm{t}$, $J=7.0 \mathrm{~Hz}, 2 \mathrm{H}), 7.08(\mathrm{~d}, J=8.0 \mathrm{~Hz}, 1 \mathrm{H}), 3.81(\mathrm{~s}, 2 \mathrm{H}), 3.28(\mathrm{~s}, 3 \mathrm{H}) .{ }^{13} \mathbf{C}$ NMR $(100$ $\left.\mathrm{MHz}, \mathrm{CDCl}_{3}\right) \delta 162.4,144.7,143.2,140.9,140.7,140.2,127.0,127.0,125.1,121.4$, 120.6, 120.0, 119.5, 37.0, 32.5. HRMS (ESI) m/z: $[\mathrm{M}+\mathrm{H}]^{+}$Calcd. for $\mathrm{C}_{15} \mathrm{H}_{14} \mathrm{NO}$ 224.1070; Found 224.1073.<smiles>CN(C=O)c1ccsc1</smiles>

$\mathrm{N}$-Methyl- $\mathrm{N}$-(thiophen-3-yl)formamide (1x) ${ }^{4}$

Yellow oil (2.5 g, 90\%). ${ }^{1} \mathbf{H}$ NMR (400 MHz, $\left.\mathrm{CDCl}_{3}\right) \delta 8.55(\mathrm{~s}, 0.8 \mathrm{H}), 8.26(\mathrm{~s}, 0.2 \mathrm{H})$, $7.46(\mathrm{dd}, J=1.6,5.2 \mathrm{~Hz}, 0.2 \mathrm{H}), 7.37(\mathrm{dd}, J=1.6,3.6 \mathrm{~Hz}, 0.2 \mathrm{H}), 7.34(\mathrm{dd}, J=3.2,5.2$ $\mathrm{Hz}, 0.8 \mathrm{H}), 7.27(\mathrm{dd}, J=3.2,5.2 \mathrm{~Hz}, 0.2 \mathrm{H}), 7.01(\mathrm{dd}, J=1.2,5.2 \mathrm{~Hz}, 0.8 \mathrm{H}), 6.86(\mathrm{dd}, J$ $=1.6,3.2 \mathrm{~Hz}, 0.8 \mathrm{H}), 3.33(\mathrm{~s}, 2.4 \mathrm{H}), 3.25(\mathrm{~s}, 0.6 \mathrm{H}) \cdot{ }^{13} \mathbf{C ~ N M R}\left(100 \mathrm{MHz}, \mathrm{CDCl}_{3}\right) \delta$ $161.7,161.2,141.2,138.7,126.8,124.6,121.5,120.9,111.7,111.6,36.5,31.9$.

\section{Ligand Synthesis}

Secondary phosphines oxides (SPO's) ligand was prepared according to the reported procedure in literature. $\mathbf{L}_{1}, \mathbf{L}_{2}, \mathbf{L}_{13}, \mathbf{L}_{14}$ and $\mathbf{L}_{16}$ were prepared according to Busacca's method. ${ }^{5} \mathbf{L}_{3}, \mathbf{L}_{\mathbf{4}}$ and $\mathbf{L}_{15}$ were prepared according to Munoz's method. ${ }^{6} \mathbf{L}_{5}, \mathbf{L}_{6}, \mathbf{L}_{7}$ and 
$\mathbf{L}_{\mathbf{8}}$ were prepared according to our previous method. ${ }^{7} \mathbf{L}_{\mathbf{9}}, \mathbf{L}_{\mathbf{1 0}}$ and $\mathbf{L}_{\mathbf{1 1}}$ were prepared according to Ackermann's method. ${ }^{8}$

Procedure for SPO $\mathrm{L}_{12}$

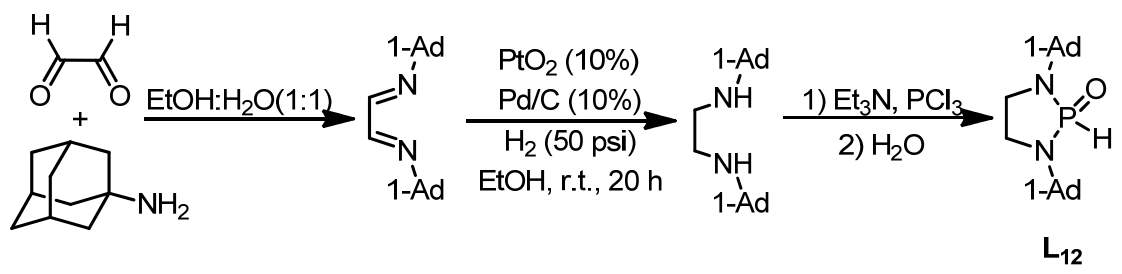

To the solution of 1-adamantlyamine $(1.5 \mathrm{~g}, 10.0 \mathrm{mmol})$ in $100 \mathrm{~mL} \mathrm{H}_{2} \mathrm{O}$ and $\mathrm{EtOH}$ (1:1) was added glyoxal $(0.72 \mathrm{~mL}$, wt. $40 \%$ in water, $5.0 \mathrm{mmol})$ dropwise. The mixture was stirred at r.t. for 20 minutes, then heated to $70{ }^{\circ} \mathrm{C}$ in an oil bath and stirred for $1 \mathrm{~h}$. The colorless solid product was collected by gravity filtration and washed with $10 \mathrm{~mL} \mathrm{H} \mathrm{H}_{2} \mathrm{O}$ :EtOH (1:1). ${ }^{\mathbf{1}} \mathbf{H}$ NMR (400 MHz, $\left.\mathbf{C D C l}_{3}\right) \delta 7.90$ (s, 2H), $2.12(\mathrm{~s}, 6 \mathrm{H}), 1.72-1.62(\mathrm{~m}, 24 \mathrm{H}) .^{9}$

$N, N^{\prime}$-(Ethane-1,2-diylidene)-bis(adamantan-1-amine) (1.0 g, $\left.3.1 \mathrm{mmol}\right)$ was suspended in $5.0 \mathrm{~mL}$ of absolute EtOH in a steel vessel. To this heterogeneous solution were added $\mathrm{Pd} / \mathrm{C}(10 \%, 0.1 \mathrm{~g})$ and platinum oxide $(0.1 \mathrm{~g})$ were added. The vessel was purged with $\mathrm{H}_{2}$ gas and stirred under a hydrogen atmosphere (50 psi) for $20 \mathrm{~h}$. Filtration through a short pad of Celite afforded a clear homogeneous solution. The solvent was concentrated in vacuo and the crude diamine was used directly in the next step without further purification.

To a solution of the diamine and $\mathrm{Et}_{3} \mathrm{~N}(5.6 \mathrm{~mL}, 8.0$ equiv. $)$ in $\mathrm{DCM}(40 \mathrm{~mL})$ at $0{ }^{\circ} \mathrm{C}$ was added dropwise $\mathrm{PCl}_{3}(5 \mathrm{~mL}, 2 \mathrm{M}$ in DCM, 2.0 equiv). The reaction mixture was warmed to $23{ }^{\circ} \mathrm{C}$ and stirred for $1 \mathrm{~h}$. Cooled to $0{ }^{\circ} \mathrm{C}$ again, the mixture was subjected to $\mathrm{H}_{2} \mathrm{O}\left(90.0 \mu \mathrm{L}, 1.0\right.$ equiv.). The resulting mixture was warmed up to $23{ }^{\circ} \mathrm{C}$ and stirred at this temperature for $1 \mathrm{~h}$. After removing all volatiles in vacuo, the residue was suspended in $\mathrm{Et}_{2} \mathrm{O}(5 \mathrm{~mL})$ and filtered through a pad of Celite eluting with $\mathrm{Et}_{2} \mathrm{O}$. The filtrate was evaporated in vacuo to afford the crude diaminophosphine oxides $\mathbf{L}_{\mathbf{1 1}}$, which were further purified by column chromatography on silica gel (50-100\% EtOAc/n-hexane).

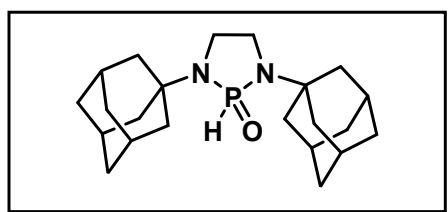

1,3-di((1s,3S)-adamantan-1-yl)-1,3,2-diazaphospholidine 2-oxide $\left(\mathrm{L}_{12}\right)$

White solid (2.9 g, 80\% of 3 steps), m.p. $261-263^{\circ} \mathrm{C} .{ }^{1} \mathbf{H}$ NMR $\left(400 \mathrm{MHz}, \mathrm{CDCl}_{3}\right): \delta$ $7.63\left(\mathrm{~d}, J_{\mathrm{P}-\mathrm{H}}=602 \mathrm{~Hz}, 1 \mathrm{H}\right), 3.35-3.23(\mathrm{~m}, 2 \mathrm{H}), 3.14-3.00(\mathrm{~m}, 2 \mathrm{H}), 2.08(\mathrm{~s}, 3 \mathrm{H})$, $1.94(\mathrm{dd}, \mathrm{J}=37.6,11.6 \mathrm{~Hz}, 12 \mathrm{H}), 1.64(\mathrm{~s}, 12 \mathrm{H}) .{ }^{13} \mathbf{C ~ N M R}\left(100 \mathrm{MHz}, \mathrm{CDCl}_{3}\right) \delta 53.2$, 53.2, 41.9, 41.8, 39.3, 39.2, 36.5, 29.7. ${ }^{31} \mathbf{P}$ NMR (162 MHz, $\left.\mathrm{CDCl}_{3}\right): \delta$ 7.23. HRMS (ESI) $\mathrm{m} / \mathrm{z}:[\mathrm{M}+\mathrm{H}]^{+}$Calcd. for $\mathrm{C}_{22} \mathrm{H}_{36} \mathrm{~N}_{2} \mathrm{OP}$ 375.2560; Found 375.2568. 


\section{Reaction Optimization}

Table S1. Ligand Effect

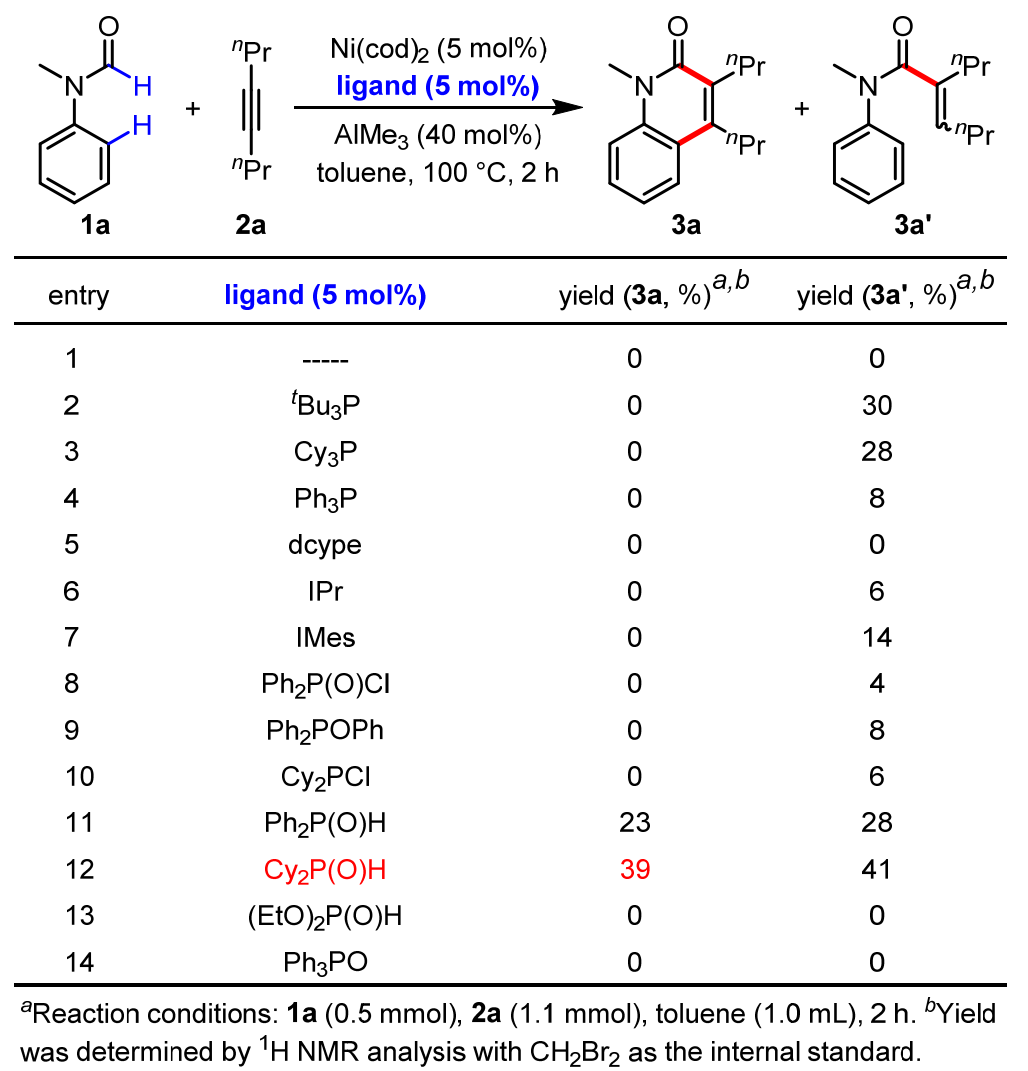

Table S2. Lewis Acid Effect

\begin{tabular}{|c|c|c|c|}
\hline $1 a$ & 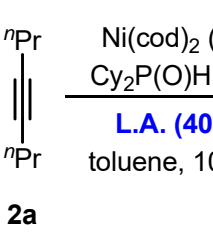 & 11 & (1) \\
\hline entry & L.A. (40 mol\%) & yield $(\mathbf{3 a}, \%)^{a, b}$ & yield $\left(3 a^{\prime}, \%\right)^{a, b}$ \\
\hline 1 & $\mathrm{AlMe}_{3}$ & 39 & 41 \\
\hline 2 & $\mathrm{AlEt}_{3}$ & 32 & 26 \\
\hline 3 & $\mathrm{Al}^{i} \mathrm{Bu}_{3}$ & 35 & 33 \\
\hline 4 & $\mathrm{AlMe}_{2} \mathrm{Cl}$ & 9 & 13 \\
\hline 5 & $\mathrm{BF}_{3} \cdot \mathrm{Et}_{2} \mathrm{O}$ & 0 & 0 \\
\hline 6 & $\mathrm{BEt}_{3}$ & 0 & 16 \\
\hline 7 & $\mathrm{BPh}_{3}$ & 15 & 14 \\
\hline 8 & $\mathrm{ZnMe}_{2}$ & trace & 37 \\
\hline 9 & $\mathrm{ZnEt}_{2}$ & 2 & 0 \\
\hline 10 & $\mathrm{ZnOTf}_{2}$ & 0 & 0 \\
\hline 11 & $\mathrm{TiCl}_{4}$ & 0 & 0 \\
\hline 12 & $\mathrm{Ti}(\mathrm{OiPr})_{4}$ & 0 & 0 \\
\hline 13 & MAD & 13 & 13 \\
\hline
\end{tabular}

${ }^{a}$ Reaction conditions: $1 \mathrm{a}(0.5 \mathrm{mmol}), \mathbf{2 a}(1.1 \mathrm{mmol})$, toluene $(1.0 \mathrm{~mL}), 2 \mathrm{~h} .{ }^{b}$ Yield was determined by ${ }^{1} \mathrm{H}$ NMR analysis with $\mathrm{CH}_{2} \mathrm{Br}_{2}$ as the internal standard. 
Table S3. Solvent Effect

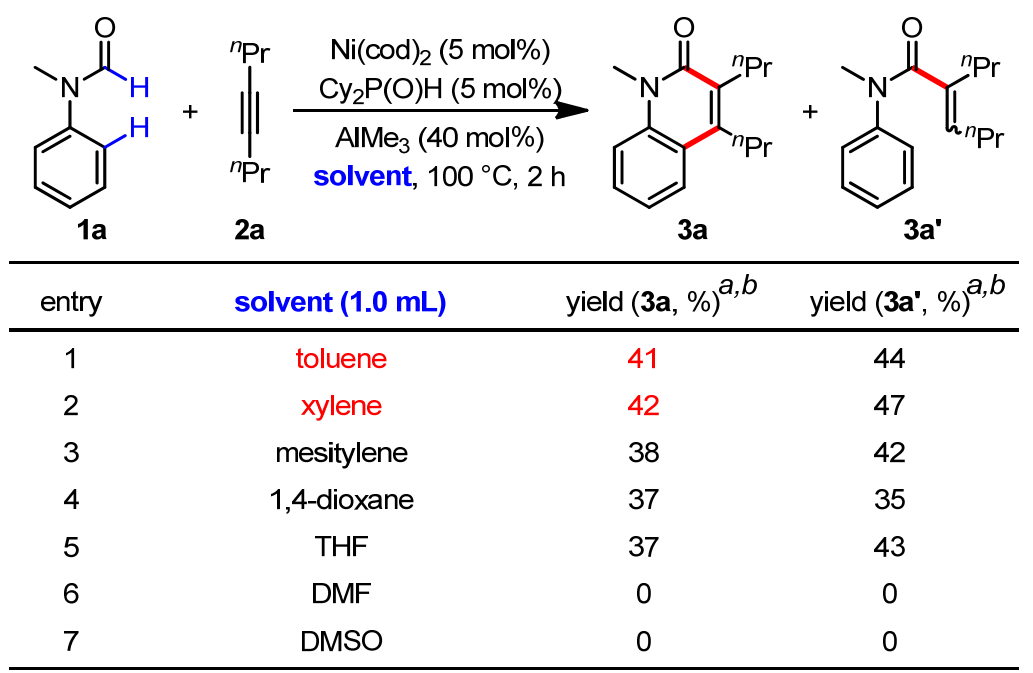

${ }^{a}$ Reaction conditions: $1 \mathrm{a}(0.5 \mathrm{mmol}), \mathbf{2 a}(1.1 \mathrm{mmol}), 2 \mathrm{~h} .{ }^{b}$ Yield was determined by ${ }^{1} \mathrm{H}$ NMR analysis with $\mathrm{CH}_{2} \mathrm{Br}_{2}$ as the internal standard.

\section{Table S4. SPO Ligand Effect}

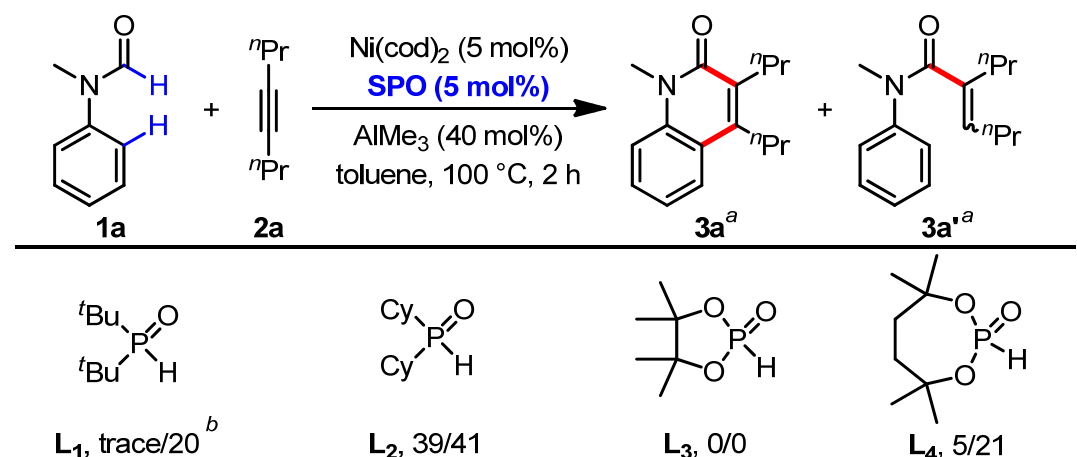

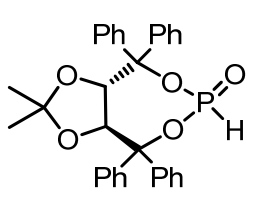<smiles>[2H]P1(=O)Oc2ccc3ccccc3c2-c2c(ccc3ccccc23)O1</smiles>

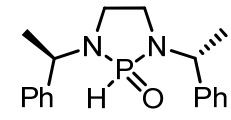<smiles>[2H]N([NH3+])P1(=O)N(C(C)C)CCN1C(C)C</smiles>

$\mathrm{L}_{5}, 10 / 0$

$\mathrm{L}_{6}, \mathrm{O} / 0$

$\mathrm{L}_{7}, 30 / 42$

$\mathrm{L}_{8}, 45 / 39$<smiles></smiles><smiles>O=P1(Br)N(Br)CCN1Br</smiles><smiles>[2H]P1(=O)N([AlH2])CCN1[TeH]</smiles>

$L_{9}, 2 / 98$

$\mathrm{L}_{10}, 20 / 31$

$\mathrm{L}_{11}, 81 / 16$

$\mathrm{L}_{12}, 70 / 22$
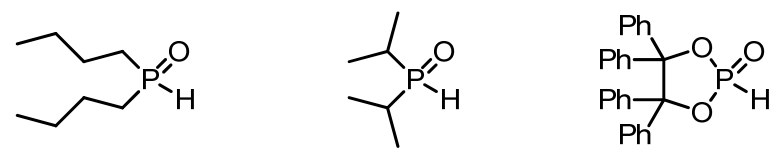<smiles></smiles>

$\mathrm{L}_{13}, 36 / 42$

$$
\mathrm{L}_{14}, 41 / 49
$$

$\mathrm{L}_{15}, 0 / 0$

$\mathrm{L}_{16}, 22 / 45$

${ }^{a}$ Reaction conditions: $1 \mathbf{a}(0.5 \mathrm{mmol}), \mathbf{2 a}(1.1 \mathrm{mmol})$, toluene $(1.0 \mathrm{~mL}), 2 \mathrm{~h} .{ }^{b}$ Yield $(\%)$ of 3a/3a' was determined by ${ }^{1} \mathrm{H}$ NMR analysis with $\mathrm{CH}_{2} \mathrm{Br}_{2}$ as the internal standard. 
Table S5. Loading Effect of Ni

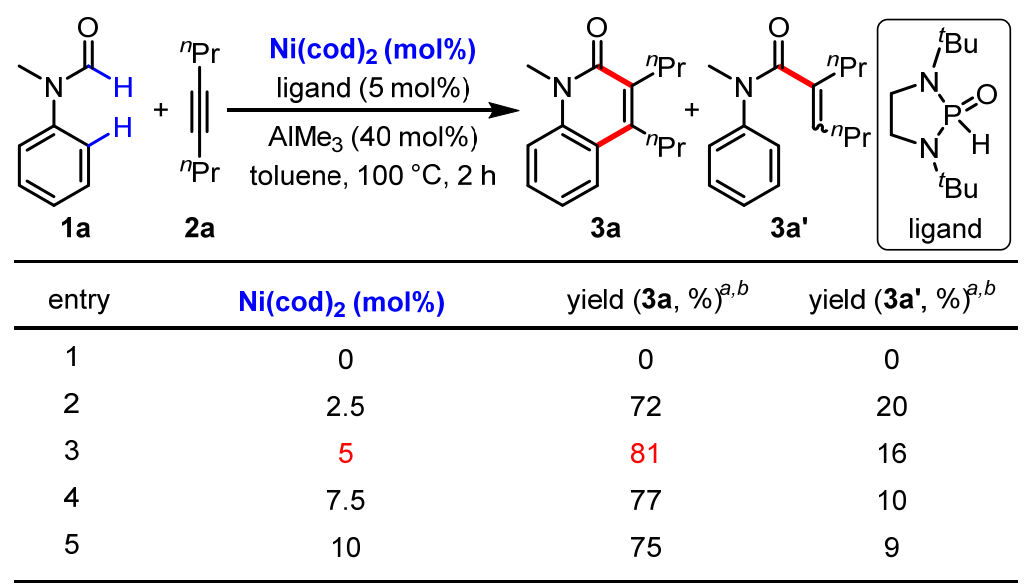

${ }^{a}$ Reaction conditions: $1 \mathrm{a}(0.5 \mathrm{mmol}), \mathbf{2 a}(1.1 \mathrm{mmol})$, toluene $(1.0 \mathrm{~mL}), 2 \mathrm{~h} .{ }^{b}$ Yield was determined by ${ }^{1} \mathrm{H}$ NMR analysis with $\mathrm{CH}_{2} \mathrm{Br}_{2}$ as the internal standard.

\section{Table S6. Loading Effect of Ligand}

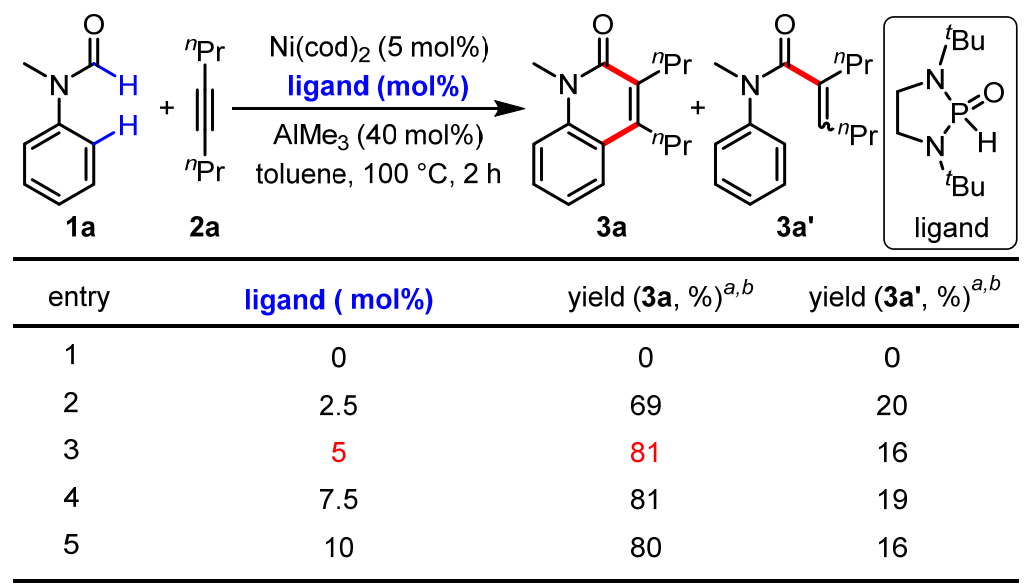

a Reaction conditions: $1 \mathrm{a}(0.5 \mathrm{mmol}), \mathbf{2 a}(1.1 \mathrm{mmol})$, toluene $(1.0 \mathrm{~mL}), 2 \mathrm{~h} .{ }^{b}$ Yield was determined by ${ }^{1} \mathrm{H}$ NMR analysis with $\mathrm{CH}_{2} \mathrm{Br}_{2}$ as the internal standard.

Table S7. Loading Effect of Alkyne

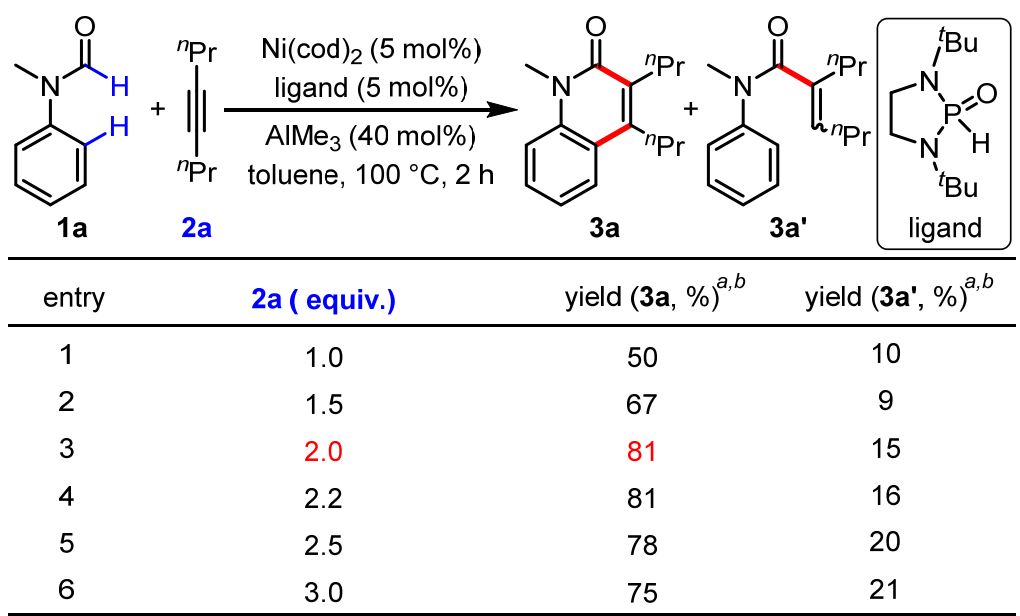

${ }^{a}$ Reaction conditions: $1 \mathrm{a}(0.5 \mathrm{mmol})$, toluene $(1.0 \mathrm{~mL}), 2 \mathrm{~h} .{ }^{b}$ Yield was determined by ${ }^{1} \mathrm{H}$ NMR analysis with $\mathrm{CH}_{2} \mathrm{Br}_{2}$ as the internal standard. 
Table S8. Temperature Effect

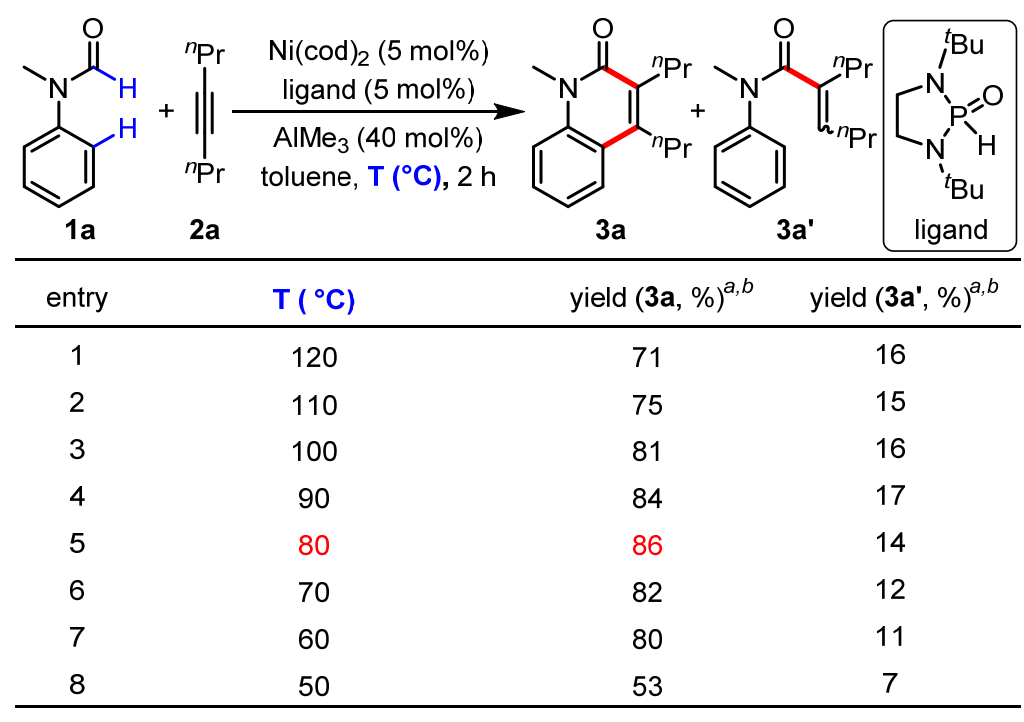

${ }^{a}$ Reaction conditions: $1 \mathbf{a}(0.5 \mathrm{mmol}), \mathbf{2 a}(1.0 \mathrm{mmol})$, toluene $(1.0 \mathrm{~mL}), 2 \mathrm{~h} .{ }^{b}$ Yield was determined by ${ }^{1} \mathrm{H}$ NMR analysis with $\mathrm{CH}_{2} \mathrm{Br}_{2}$ as the internal standard.

\section{Table S9. Concentration Effect}

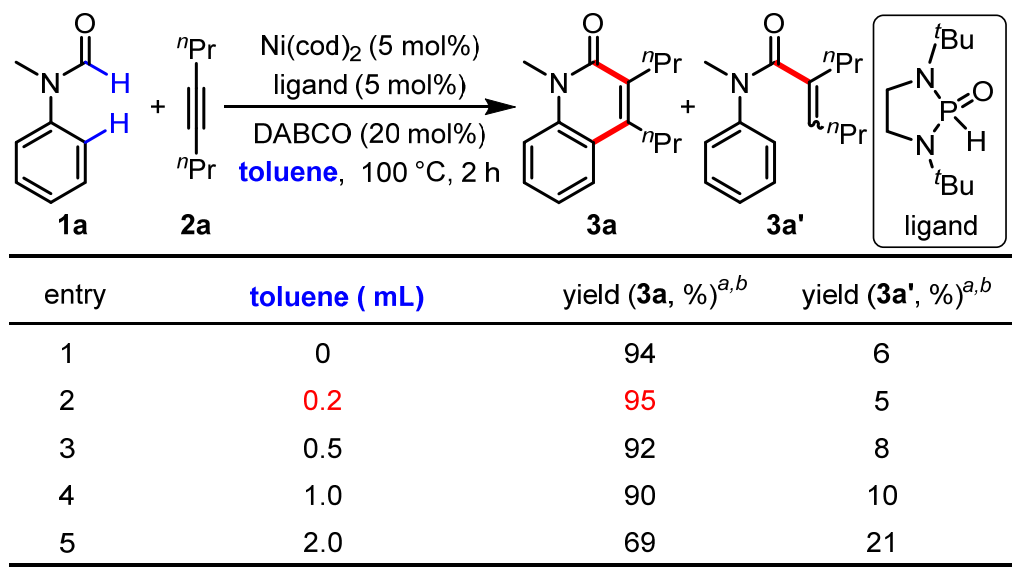

${ }^{a}$ Reaction conditions: $1 \mathrm{a}(0.5 \mathrm{mmol}), \mathbf{2 a}(1.0 \mathrm{mmol}), 2 \mathrm{~h} .{ }^{b}$ Yield was determined by ${ }^{1} \mathrm{H}$ NMR analysis with $\mathrm{CH}_{2} \mathrm{Br}_{2}$ as the internal standard.

\section{Typical Procedure for Ni-Catalyzed C-H Annulation}

To a $15 \mathrm{~mL}$ oven-dried tube were added SPO ligand $\mathbf{L}_{11}(5.5 \mathrm{mg}, 5 \mathrm{~mol} \%)$, $\mathrm{Ni}(\operatorname{cod})_{2}(6.9 \mathrm{mg}, 5 \mathrm{~mol} \%)$, dry degassed toluene $(0.2 \mathrm{~mL})$, arylformamide $(0.5 \mathrm{mmol})$, $\mathrm{AlMe}_{3}(200 \mu \mathrm{L}, 40 \mathrm{~mol} \%)$ and alkyne $(1.0 \mathrm{mmol})$ sequentially in an $\mathrm{N}_{2}$-filled glove-box. The tube was sealed and removed out of the glove-box. After heated at $80{ }^{\circ} \mathrm{C}$ in a dry block heater for $2 \mathrm{~h}$, the mixture was cooled to r.t., quenched with 0.1 $\mathrm{mL}$ of 5\% EDTA disodium salt solution, filtered through a short plug of silica gel (EtOAc as the eluent) and concentrated in vacuo to afford the crude product. Further purification by flash column chromatography on silica gel (eluting with EtOAc/ $n$-hexane) gave the pure product. 


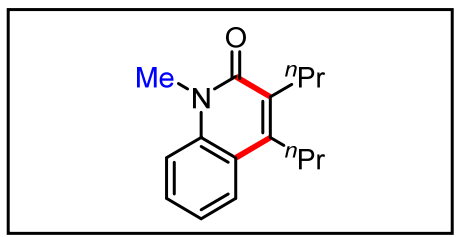

1-Methyl-3,4-dipropylquinolin-2(1H)-one (3a) ${ }^{10}$

White solid (109.4 mg, 90\%), m.p. 71-73 ${ }^{\circ} \mathrm{C} .{ }^{1} \mathbf{H}$ NMR (400 MHz, $\left.\mathrm{CDCl}_{3}\right) \delta 7.74(\mathrm{~d}, J$ $=8.0 \mathrm{~Hz}, 1 \mathrm{H}), 7.50(\mathrm{t}, J=7.8 \mathrm{~Hz}, 1 \mathrm{H}), 7.35(\mathrm{~d}, J=8.4 \mathrm{~Hz}, 1 \mathrm{H}), 7.23(\mathrm{t}, J=7.8 \mathrm{~Hz}, 1 \mathrm{H})$, $3.74(\mathrm{~s}, 3 \mathrm{H}), 2.93-2.82(\mathrm{~m}, 2 \mathrm{H}), 2.78-2.65(\mathrm{~m}, 2 \mathrm{H}), 1.75-1.49(\mathrm{~m}, 4 \mathrm{H}), 1.10(\mathrm{t}, J=$ $7.2 \mathrm{~Hz}, 3 \mathrm{H}), 1.04(\mathrm{t}, \mathrm{J}=7.2 \mathrm{~Hz}, 3 \mathrm{H}) .{ }^{13} \mathbf{C}$ NMR $\left(100 \mathrm{MHz}, \mathrm{CDCl}_{3}\right) \delta 162.3,145.1$, 138.9, 131.4, 129.2, 125.1, 121.8, 120.7, 114.4, 30.9, 30.2, 29.9, 23.4, 22.7, 14.7, 14.7.

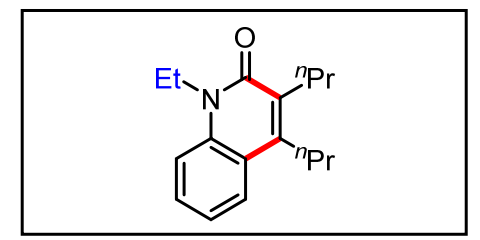

\section{1-Ethyl-3,4-dipropylquinolin-2(1H)-one $(3 \mathrm{~b})^{10}$}

Colorless oil (115.7 mg, 90\%). ${ }^{1} \mathbf{H}$ NMR $\left(400 \mathrm{MHz}, \mathrm{CDCl}_{3}\right) \delta 7.74(\mathrm{~d}, J=8.0 \mathrm{~Hz}, 1 \mathrm{H})$, $7.49(\mathrm{t}, J=7.8 \mathrm{~Hz}, 1 \mathrm{H}), 7.37(\mathrm{~d}, J=8.4 \mathrm{~Hz}, 1 \mathrm{H}), 7.22(\mathrm{t}, J=7.6 \mathrm{~Hz}, 1 \mathrm{H}), 4.38$ (q, $J=7.0$ $\mathrm{Hz}, 2 \mathrm{H}), 2.93-2.81(\mathrm{~m}, 2 \mathrm{H}), 2.75-2.65(\mathrm{~m}, 2 \mathrm{H}), 1.72-1.50(\mathrm{~m}, 4 \mathrm{H}), 1.36(\mathrm{t}, J=7.0$ $\mathrm{Hz}, 3 \mathrm{H}), 1.10(\mathrm{t}, J=7.4 \mathrm{~Hz}, 3 \mathrm{H}), 1.04$ (t, $J=7.4 \mathrm{~Hz}, 3 \mathrm{H}) .{ }^{13} \mathbf{C} \mathbf{N M R}\left(100 \mathrm{MHz}, \mathrm{CDCl}_{3}\right)$ $\delta 161.7,145.0,137.9,131.5,129.1,125.3,121.6,121.0,114.2,37.7,31.0,30.1,23.4$, $22.7,14.7,14.7,12.9$.

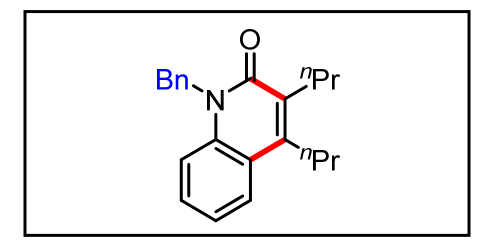

1-Benzyl-3,4-dipropylquinolin-2(1H)-one $(3 \mathrm{c})^{11}$

Colorless oil (138.8 mg, 87\%). ${ }^{1} \mathbf{H}$ NMR $\left(400 \mathrm{MHz}, \mathrm{CDCl}_{3}\right) \delta 7.74(\mathrm{~d}, J=8.0 \mathrm{~Hz}, 1 \mathrm{H})$, $7.38-7.32(\mathrm{~m}, 1 \mathrm{H}), 7.31-7.27(\mathrm{~m}, 2 \mathrm{H}), 7.25-7.15(\mathrm{~m}, 4 \mathrm{H}), 5.58(\mathrm{~s}, 2 \mathrm{H}), 2.93-2.85$ $(\mathrm{m}, 2 \mathrm{H}), 2.81-2.72(\mathrm{~m}, 2 \mathrm{H}), 1.74-1.65(\mathrm{~m}, 2 \mathrm{H}), 1.64-1.55(\mathrm{~m}, 2 \mathrm{H}), 1.13(\mathrm{t}, J=7.4$ $\mathrm{Hz}, 3 \mathrm{H}), 1.06(\mathrm{t}, J=7.2 \mathrm{~Hz}, 3 \mathrm{H}) .{ }^{13} \mathbf{C} \mathbf{~ N M R}\left(100 \mathrm{MHz}, \mathrm{CDCl}_{3}\right) \delta 162.4,145.6,138.3$, $136.9,131.4,129.1,128.8,127.1,126.7,125.1,121.9,121.0,115.2,46.4,31.1,30.2$, $23.4,22.8,14.7,14.7$.

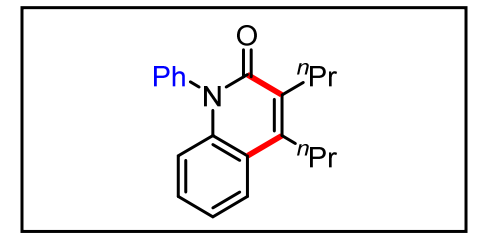

\section{1-Phenyl-3,4-dipropylquinolin-2(1H)-one (3d) ${ }^{10}$}

White solid (109.9 mg, 72\%), m.p. $71-73{ }^{\circ} \mathrm{C} .{ }^{\mathbf{1}} \mathbf{H}$ NMR $\left(400 \mathrm{MHz}, \mathrm{CDCl}_{3}\right) \delta 7.76(\mathrm{~d}, J$ $=8.0,1 \mathrm{H}), 7.61-7.54(\mathrm{~m}, 2 \mathrm{H}), 7.52-7.46(\mathrm{~m}, 1 \mathrm{H}), 7.31-7.16(\mathrm{~m}, 4 \mathrm{H}), 6.66(\mathrm{~d}, J=$ $8.4 \mathrm{~Hz}, 1 \mathrm{H}), 2.98-2.89(\mathrm{~m}, 2 \mathrm{H}), 2.77-2.69(\mathrm{~m}, 2 \mathrm{H}), 1.81-1.69(\mathrm{~m}, 2 \mathrm{H}), 1.67-1.56$ 
$(\mathrm{m}, 2 \mathrm{H}), 1.16(\mathrm{t}, J=7.4 \mathrm{~Hz}, 3 \mathrm{H}), 1.03(\mathrm{t}, J=7.4 \mathrm{~Hz}, 3 \mathrm{H}) .{ }^{13} \mathbf{C}$ NMR $\left(100 \mathrm{MHz}, \mathrm{CDCl}_{3}\right)$ $\delta 162.4,146.0,140.0,138.5,131.9,130.1,129.0,128.7,128.6,124.8,122.1,120.5$, $116.3,31.1,30.0,23.4,22.7,14.8,14.7$.

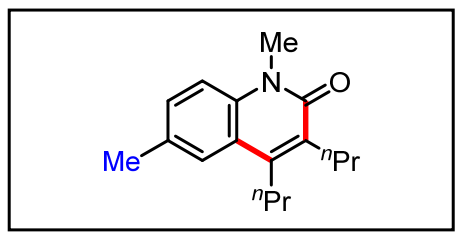

\section{1,6-Dimethyl-3,4-dipropylquinolin-2(1H)-one (3e) ${ }^{10}$}

White solid (92.6 mg, 72\%), m.p. 76-78 ${ }^{\circ} \mathrm{C} .{ }^{1} \mathbf{H}$ NMR (400 MHz, $\left.\mathrm{CDCl}_{3}\right) \delta 7.50(\mathrm{~s}, 1 \mathrm{H})$, $7.31(\mathrm{~d}, J=8.6 \mathrm{~Hz}, 1 \mathrm{H}), 7.24(\mathrm{~d}, J=8.6 \mathrm{~Hz}, 1 \mathrm{H}), 3.72(\mathrm{~s}, 3 \mathrm{H}), 2.89-2.79(\mathrm{~m}, 2 \mathrm{H}), 2.75$ $-2.65(\mathrm{~m}, 2 \mathrm{H}), 2.45(\mathrm{~s}, 3 \mathrm{H}), 1.73-1.49(\mathrm{~m}, 4 \mathrm{H}), 1.10(\mathrm{t}, J=7.4 \mathrm{~Hz}, 3 \mathrm{H}), 1.03(\mathrm{t}, J=$ $7.4 \mathrm{~Hz}, 3 \mathrm{H}) .{ }^{13} \mathbf{C}$ NMR $\left(100 \mathrm{MHz}, \mathrm{CDCl}_{3}\right) \delta 162.2,144.9,136.9,131.4,131.1,130.3$, $125.0,120.7,114.3,30.9,30.2,29.9,23.4,22.7,21.2,14.7,14.7$.

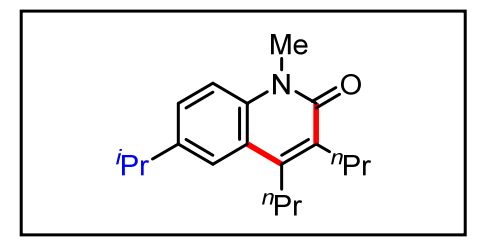

6-Isopropyl-1-methyl-3,4-dipropylquinolin-2(1H)-one (3f)

White solid (128.3 mg, 90\%), m.p. 46-48 ${ }^{\circ} \mathrm{C} .{ }^{1} \mathbf{H}$ NMR (400 MHz, $\left.\mathrm{CDCl}_{3}\right) \delta 7.55$ (d, $J$ $=1.6 \mathrm{~Hz}, 1 \mathrm{H}), 7.39(\mathrm{dd}, J=8.8,2.0 \mathrm{~Hz}, 1 \mathrm{H}), 7.29(\mathrm{~d}, J=8.4 \mathrm{~Hz}, 1 \mathrm{H}), 3.73(\mathrm{~s}, 3 \mathrm{H}), 3.06$ $-2.95(\mathrm{~m}, 1 \mathrm{H}), 2.90-2.83(\mathrm{~m}, 2 \mathrm{H}), 2.74-2.67(\mathrm{~m}, 2 \mathrm{H}), 1.71-1.62(\mathrm{~m}, 2 \mathrm{H}), 1.61-$ $1.52(\mathrm{~m}, 2 \mathrm{H}), 1.31(\mathrm{~d}, J=6.8 \mathrm{~Hz}, 6 \mathrm{H}), 1.10(\mathrm{t}, J=7.4 \mathrm{~Hz}, 3 \mathrm{H}), 1.03(\mathrm{t}, J=7.4 \mathrm{~Hz}, 3 \mathrm{H})$. ${ }^{13}$ C NMR (100 MHz, $\left.\mathrm{CDCl}_{3}\right) \delta 162.2,145.0,142.2,137.2,131.4,127.7,122.5,120.6$, 114.4, 33.8, 30.9, 30.2, 29.9, 24.3, 23.4, 22.7, 14.7, 14.7. HRMS (ESI) m/z: $[\mathrm{M}+\mathrm{H}]^{+}$ Calcd. for $\mathrm{C}_{19} \mathrm{H}_{28} \mathrm{NO} 286.2165$; Found 286.2170.

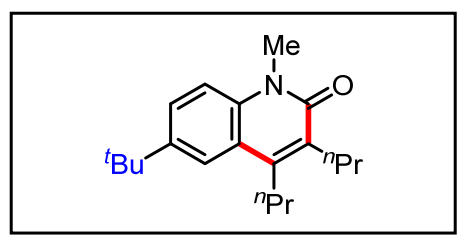

\section{6-(tert-Butyl)-1-methyl-3,4-dipropylquinolin-2(1H)-one (3g)}

White solid (136.1 mg, 91\%), m.p. 66-68 ${ }^{\circ} \mathrm{C} .{ }^{1} \mathbf{H}$ NMR (400 MHz, $\left.\mathrm{CDCl}_{3}\right) \delta 7.72$ (d, $J$ $=2.0 \mathrm{~Hz}, 1 \mathrm{H}), 7.55(\mathrm{dd}, J=8.8,2.4 \mathrm{~Hz}, 1 \mathrm{H}), 7.30(\mathrm{~d}, J=8.8 \mathrm{~Hz}, 1 \mathrm{H}), 3.73(\mathrm{~s}, 3 \mathrm{H}), 2.93$ $-2.85(\mathrm{~m}, 2 \mathrm{H}), 2.75-2.67(\mathrm{~m}, 2 \mathrm{H}), 1.72-1.63(\mathrm{~m}, 2 \mathrm{H}), 1.61-1.51(\mathrm{~m}, 2 \mathrm{H}), 1.38(\mathrm{~s}$, 9H), $1.11(\mathrm{t}, J=7.4 \mathrm{~Hz}, 3 \mathrm{H}), 1.03(\mathrm{t}, J=7.4 \mathrm{~Hz}, 3 \mathrm{H}) .{ }^{13} \mathbf{C ~ N M R}\left(100 \mathrm{MHz}, \mathrm{CDCl}_{3}\right) \delta$ $162.3,145.2,144.4,136.8,131.4,126.8,121.3,120.2,114.1,34.5,31.5,30.9,30.2$, 29.8, 23.4, 22.8, 14.7, 14.7. HRMS (ESI) m/z: $[\mathrm{M}+\mathrm{H}]^{+}$Calcd. for $\mathrm{C}_{20} \mathrm{H}_{30} \mathrm{NO} 300.2322$; Found 300.2324. 


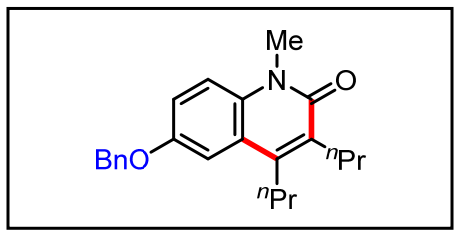

6-(Benzyloxy)-1-methyl-3,4-dipropylquinolin-2(1H)-one (3h)

White solid (157.1 mg, 90\%), m.p. 95-97 ${ }^{\circ} \mathrm{C} .{ }^{1} \mathbf{H}$ NMR (400 MHz, $\left.\mathrm{CDCl}_{3}\right) \delta 7.46(\mathrm{~d}, J$ $=7.6 \mathrm{~Hz}, 2 \mathrm{H}), 7.40(\mathrm{t}, J=7.2 \mathrm{~Hz}, 2 \mathrm{H}), 7.36-7.31(\mathrm{~m}, 1 \mathrm{H}), 7.27(\mathrm{~d}, J=8.8 \mathrm{~Hz}, 1 \mathrm{H})$, $7.20(\mathrm{~m}, 2 \mathrm{H}), 5.15(\mathrm{~s}, 2 \mathrm{H}), 3.71(\mathrm{~s}, 3 \mathrm{H}), 2.79-2.72(\mathrm{~m}, 2 \mathrm{H}), 2.71-2.65(\mathrm{~m}, 2 \mathrm{H}), 1.62$ - $1.48(\mathrm{~m}, 4 \mathrm{H}), 1.03(\mathrm{~m}, 6 \mathrm{H}) . .{ }^{13} \mathbf{C}$ NMR $\left(100 \mathrm{MHz}, \mathrm{CDCl}_{3}\right) \delta 161.8,153.6,144.4$, 137.0, 133.6, 132.1, 128.8, 128.2, 127.5, 121.6, 117.6, 115.4, 109.9, 70.7, 31.1, 30.3, 30.0, 23.1, 22.7, 14.8, 14.7. HRMS (ESI) m/z: $[\mathrm{M}+\mathrm{H}]^{+}$Calcd. for $\mathrm{C}_{23} \mathrm{H}_{28} \mathrm{NO}_{2}$ 350.2115; Found 350.2116.

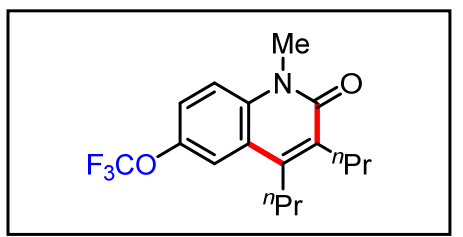

1-Methyl-3,4-dipropyl-6-(trifluoromethoxy)quinolin-2(1H)-one (3i)

White solid (140.7 mg, 86\%), m.p. 57-59 ${ }^{\circ} \mathrm{C} .{ }^{1} \mathbf{H}$ NMR $\left(400 \mathrm{MHz}, \mathrm{CDCl}_{3}\right) \delta 7.53$ (s, $1 \mathrm{H}), 7.38-7.31(\mathrm{~m}, 2 \mathrm{H}), 3.72(\mathrm{~s}, 3 \mathrm{H}), 2.85-2.77(\mathrm{~m}, 2 \mathrm{H}), 2.73-2.66(\mathrm{~m}, 2 \mathrm{H}), 1.67-$ $1.60(\mathrm{~m}, 2 \mathrm{H}), 1.58-1.50(\mathrm{~m}, 2 \mathrm{H}), 1.09(\mathrm{t}, J=7.4 \mathrm{~Hz}, 3 \mathrm{H}), 1.03(\mathrm{t}, J=7.4 \mathrm{~Hz}, 3 \mathrm{H}) .{ }^{13} \mathbf{C}$ NMR $\left(100 \mathrm{MHz}, \mathrm{CDCl}_{3}\right) \delta 162.0,144.3,143.7$ (q, $\left.J=18.6 \mathrm{~Hz}\right), 137.5,133.1,124.5$, $122.2,121.6,120.7$ (q, $J=256 \mathrm{~Hz}), 117.6,115.6,30.9,30.3,30.2,23.3,22.6,14.7,14.6$. ${ }^{19} \mathbf{F}$ NMR (376 MHz, $\mathrm{CDCl}_{3}$ ) $\delta$-58.1. HRMS (ESI) $\mathrm{m} / \mathrm{z}$ : $[\mathrm{M}+\mathrm{H}]^{+}$Calcd. for $\mathrm{C}_{17} \mathrm{H}_{21} \mathrm{~F}_{3} \mathrm{NO}_{2}$ 328.1519; Found 328.1521.

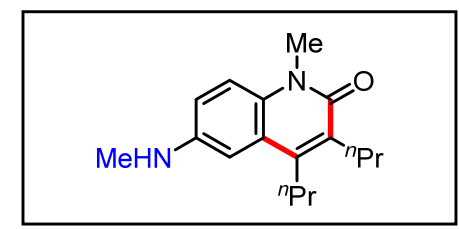

\section{1-Methyl-6-(methylamino)-3,4-dipropylquinolin-2(1H)-one (3j)}

Yellow solid (83.0 mg, 61\%), m.p. $103-105^{\circ} \mathrm{C} .{ }^{1} \mathbf{H}$ NMR $\left(400 \mathrm{MHz}, \mathrm{CDCl}_{3}\right) \delta 7.21(\mathrm{~d}$, $J=9.6 \mathrm{~Hz}, 1 \mathrm{H}), 6.87(\mathrm{~d}, J=6.0 \mathrm{~Hz}, 2 \mathrm{H}), 3.70(\mathrm{~s}, 3 \mathrm{H}), 2.90(\mathrm{~s}, 3 \mathrm{H}), 2.85-2.78(\mathrm{~m}, 2 \mathrm{H})$, $2.74-2.63(\mathrm{~m}, 2 \mathrm{H}), 1.70-1.61(\mathrm{~m}, 2 \mathrm{H}), 1.62-1.50(\mathrm{~m}, 2 \mathrm{H}), 1.09(\mathrm{t}, J=7.2 \mathrm{~Hz}, 3 \mathrm{H})$, $1.03(\mathrm{t}, J=7.2 \mathrm{~Hz}, 3 \mathrm{H}) .{ }^{13} \mathrm{C}$ NMR $\left(100 \mathrm{MHz}, \mathrm{CDCl}_{3}\right) \delta 161.6,144.4,144.4,131.6$, 131.6, 121.7, 116.2, 115.3, 106.1, 31.3, 31.0, 30.3, 29.8, 23.1, 22.8, 14.8, 14.7. HRMS (ESI) m/z: $[\mathrm{M}+\mathrm{H}]^{+}$Calcd. for $\mathrm{C}_{17} \mathrm{H}_{25} \mathrm{~N}_{2} \mathrm{O} 273.1961$; Found 273.1961.

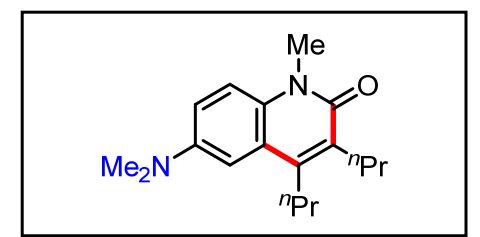

6-(Dimethylamino)-1-methyl-3,4-dipropylquinolin-2(1H)-one (3k) 
Yellow solid (107.3 mg, 75\%), m.p. $126-128^{\circ} \mathrm{C} .{ }^{1} \mathbf{H}$ NMR $\left(400 \mathrm{MHz}, \mathrm{CDCl}_{3}\right) \delta 7.28(\mathrm{~s}$, $1 \mathrm{H}), 7.14-6.90(\mathrm{~m}, 2 \mathrm{H}), 3.71(\mathrm{~s}, 3 \mathrm{H}), 2.99(\mathrm{~s}, 6 \mathrm{H}), 2.89-2.80(\mathrm{~m}, 2 \mathrm{H}), 2.74-2.66(\mathrm{~m}$, $2 \mathrm{H}), 1.73-1.62(\mathrm{~m}, 2 \mathrm{H}), 1.61-1.52(\mathrm{~m}, 2 \mathrm{H}), 1.10(\mathrm{t}, J=7.4 \mathrm{~Hz}, 3 \mathrm{H}), 1.03(\mathrm{t}, J=7.4$ $\mathrm{Hz}, 3 \mathrm{H}) .{ }^{13} \mathbf{C}$ NMR $\left(100 \mathrm{MHz}, \mathrm{CDCl}_{3}\right) \delta 161.7,144.6,131.8,121.5,116.7,115.2,41.7$, 31.0, 30.3, 29.8, 23.1, 22.8, 14.8, 14.7. HRMS (ESI) $\mathrm{m} / \mathrm{z}:[\mathrm{M}+\mathrm{H}]^{+}$Calcd. for $\mathrm{C}_{18} \mathrm{H}_{27} \mathrm{~N}_{2} \mathrm{O} 287.2118$; Found 287.2115.

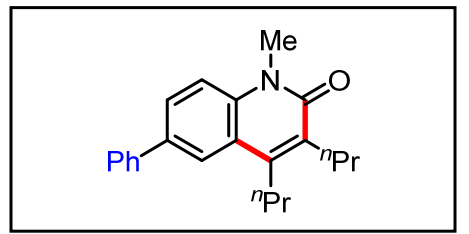

\section{1-Methyl-6-phenyl-3,4-dipropylquinolin-2(1H)-one (3I)}

White solid (143.6 mg, 90\%), m.p. 100-102 ${ }^{\circ} \mathrm{C} .{ }^{1} \mathbf{H}$ NMR (400 MHz, $\left.\mathrm{CDCl}_{3}\right) \delta 7.92(\mathrm{~d}$, $J=1.2 \mathrm{~Hz}, 1 \mathrm{H}), 7.73(\mathrm{dd}, J=8.4,1.6 \mathrm{~Hz}, 1 \mathrm{H}), 7.62(\mathrm{~d}, J=7.6 \mathrm{~Hz}, 2 \mathrm{H}), 7.49$ (t, $J=7.6$ $\mathrm{Hz}, 2 \mathrm{H}), 7.40(\mathrm{t}, J=8.8 \mathrm{~Hz}, 2 \mathrm{H}), 3.77(\mathrm{~s}, 3 \mathrm{H}), 2.97-2.88(\mathrm{~m}, 2 \mathrm{H}), 2.80-2.71(\mathrm{~m}, 2 \mathrm{H})$, $1.78-1.68(\mathrm{~m}, 2 \mathrm{H}), 1.67-1.56(\mathrm{~m}, 2 \mathrm{H}), 1.12(\mathrm{t}, J=7.4 \mathrm{~Hz}, 3 \mathrm{H}), 1.06(\mathrm{t}, J=7.4 \mathrm{~Hz}$, $3 \mathrm{H}) .{ }^{13} \mathrm{C}$ NMR $\left(100 \mathrm{MHz}, \mathrm{CDCl}_{3}\right) \delta 162.3,145.1,140.8,138.3,134.9,132.0,129.1$, 128.2, 127.4, 127.2, 123.5, 121.1, 114.9, 30.9, 30.3, 30.0, 23.5, 22.7, 14.8, 14.7. HRMS (ESI) m/z: [M+H] $]^{+}$Calcd. for $\mathrm{C}_{22} \mathrm{H}_{26} \mathrm{NO} 320.2009$; Found 320.2009.

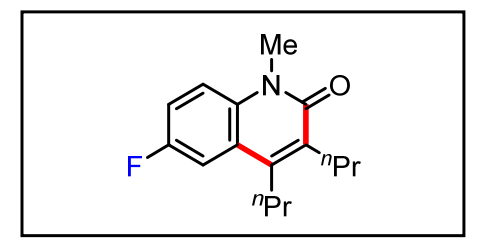

\section{6-Fluoro-1-methyl-3,4-dipropylquinolin-2(1H)-one (3m)}

White solid (87.5 mg, 67\%), m.p. $77-79^{\circ} \mathrm{C} .{ }^{1} \mathbf{H}$ NMR $\left(400 \mathrm{MHz}, \mathrm{CDCl}_{3}\right) \delta 7.39(\mathrm{dd}, J$ $=10.2,2.6 \mathrm{~Hz}, 1 \mathrm{H}), 7.30(\mathrm{dd}, J=9.2,4.8 \mathrm{~Hz}, 1 \mathrm{H}), 7.26-7.20(\mathrm{~m}, 1 \mathrm{H}), 3.73(\mathrm{~s}, 3 \mathrm{H})$, $2.83-2.75(\mathrm{~m}, 2 \mathrm{H}), 2.74-2.66(\mathrm{~m}, 2 \mathrm{H}), 1.70-1.52(\mathrm{~m}, 4 \mathrm{H}), 1.10(\mathrm{t}, J=7.4 \mathrm{~Hz}, 3 \mathrm{H})$, $1.04(\mathrm{t}, J=7.4 \mathrm{~Hz}, 3 \mathrm{H}) .{ }^{13} \mathbf{C} \mathbf{N M R}\left(100 \mathrm{MHz}, \mathrm{CDCl}_{3}\right) \delta 161.9,158.0(\mathrm{~d}, J=238 \mathrm{~Hz})$, $144.1(\mathrm{~d}, J=3.2 \mathrm{~Hz}), 135.5,132.9,121.9(\mathrm{~d}, J=7.6 \mathrm{~Hz}), 116.7(\mathrm{~d}, J=23.6 \mathrm{~Hz})$, $115.8(\mathrm{~d}, J=8.2 \mathrm{~Hz}), 110.5(\mathrm{~d}, J=23.4 \mathrm{~Hz}), 31.1,30.3,30.2,23.2,22.7,14.7 .{ }^{19} \mathbf{F}$ NMR $\left(376 \mathrm{MHz}, \mathrm{CDCl}_{3}\right) \delta$-121.4. HRMS (ESI) m/z: $[\mathrm{M}+\mathrm{H}]^{+}$Calcd. for $\mathrm{C}_{16} \mathrm{H}_{21} \mathrm{FNO}$ 262.1602; Found 262.1607.

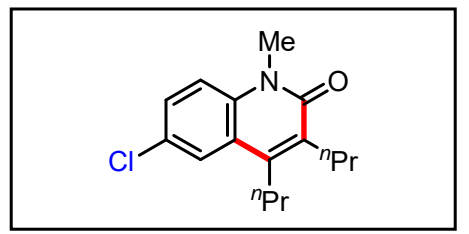

\section{6-Chloro-1-methyl-3,4-dipropylquinolin-2(1H)-one (3n) ${ }^{10}$}

White solid (94.2 mg, 68\%), m.p. 75-76 ${ }^{\circ} \mathrm{C} .{ }^{1} \mathbf{H}$ NMR (400 MHz, $\left.\mathrm{CDCl}_{3}\right) \delta 7.64(\mathrm{~d}, J=$ $2.0 \mathrm{~Hz}, 1 \mathrm{H}), 7.42(\mathrm{dd}, J=8.8,2.0 \mathrm{~Hz}, 1 \mathrm{H}), 7.26(\mathrm{~d}, J=8.8 \mathrm{~Hz}, 1 \mathrm{H}), 3.70(\mathrm{~s}, 3 \mathrm{H}), 2.83$ $-2.75(\mathrm{~m}, 2 \mathrm{H}), 2.71-2.65(\mathrm{~m}, 2 \mathrm{H}), 1.68-1.59(\mathrm{~m}, 2 \mathrm{H}), 1.58-1.49(\mathrm{~m}, 2 \mathrm{H}), 1.09(\mathrm{t}, J$ $=7.4 \mathrm{~Hz}, 3 \mathrm{H}), 1.02(\mathrm{t}, J=7.3 \mathrm{~Hz}, 3 \mathrm{H}) .{ }^{13} \mathbf{C} \mathbf{N M R}\left(100 \mathrm{MHz}, \mathrm{CDCl}_{3}\right) \delta 162.0,144.1$, 137.5, 132.8, 129.1, 127.4, 124.5, 122.0, 115.8, 30.9, 30.3, 30.1, 23.3, 22.6, 14.7, 14.7. 


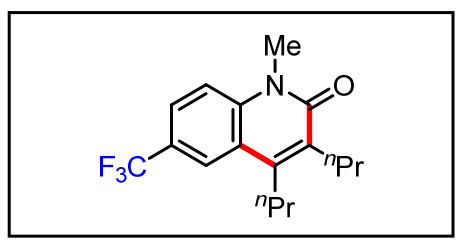

1-Methyl-3,4-dipropyl-6-(trifluoromethyl)quinolin-2(1H)-one (3o)

White solid (124.5 mg, 80\%), m.p. 89-91 ${ }^{\circ} \mathrm{C} .{ }^{1} \mathbf{H}$ NMR $\left(400 \mathrm{MHz}, \mathrm{CDCl}_{3}\right) \delta 7.94(\mathrm{~s}$, 1H), $7.69(\mathrm{dd}, J=8.4,1.2 \mathrm{~Hz}, 1 \mathrm{H}), 7.43(\mathrm{~d}, J=8.8 \mathrm{~Hz}, 1 \mathrm{H}), 3.74(\mathrm{~s}, 3 \mathrm{H}), 2.90-2.82(\mathrm{~m}$, $2 \mathrm{H}), 2.72-2.64(\mathrm{~m}, 2 \mathrm{H}), 1.68-1.61(\mathrm{~m}, 2 \mathrm{H}), 1.59-1.51(\mathrm{~m}, 2 \mathrm{H}), 1.10(\mathrm{t}, J=7.4 \mathrm{~Hz}$, $3 \mathrm{H}), 1.03(\mathrm{t}, J=7.4 \mathrm{~Hz}, 3 \mathrm{H}) .{ }^{13} \mathbf{C} \mathbf{N M R}\left(100 \mathrm{MHz}, \mathrm{CDCl}_{3}\right) \delta 162.2,144.8,141.0,133.0$, $125.5(\mathrm{q}, J=3.4 \mathrm{~Hz}), 124.4(\mathrm{q}, J=270 \mathrm{~Hz}), 123.9(\mathrm{q}, J=32.6 \mathrm{~Hz}), 122.4(\mathrm{q}, J=4.0 \mathrm{~Hz})$, $120.5,114.8,30.8,30.3,30.2,23.4,22.6,14.6,14.6 .{ }^{19} \mathbf{F ~ N M R}\left(376 \mathrm{MHz}, \mathrm{CDCl}_{3}\right) \delta$ -61.6. HRMS (ESI) m/z: $[\mathrm{M}+\mathrm{H}]^{+}$Calcd. for $\mathrm{C}_{17} \mathrm{H}_{21} \mathrm{FNO} 312.1570$; Found 312.1572.

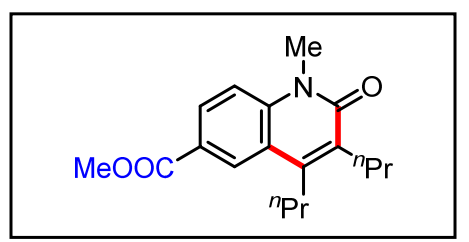

Methyl 1-methyl-2-oxo-3,4-dipropyl-1,2-dihydroquinoline-6-carboxylate (3p) White solid (106.9 mg, 71\%), m.p. 90-92 ${ }^{\circ} \mathrm{C} .{ }^{1} \mathbf{H}$ NMR $\left(400 \mathrm{MHz}, \mathrm{CDCl}_{3}\right) \delta 8.41(\mathrm{~s}$, $1 \mathrm{H}), 8.10(\mathrm{~d}, J=8.8 \mathrm{~Hz}, 1 \mathrm{H}), 7.34(\mathrm{~d}, J=8.8 \mathrm{~Hz}, 1 \mathrm{H}), 3.94(\mathrm{~s}, 3 \mathrm{H}), 3.73(\mathrm{~s}, 3 \mathrm{H}), 2.93-$ $2.84(\mathrm{~m}, 2 \mathrm{H}), 2.73-2.65(\mathrm{~m}, 2 \mathrm{H}), 1.71-1.61(\mathrm{~m}, 2 \mathrm{H}), 1.60-1.50(\mathrm{~m}, 2 \mathrm{H}), 1.10(\mathrm{t}, J=$ $7.4 \mathrm{~Hz}, 3 \mathrm{H}), 1.02(\mathrm{t}, J=7.4 \mathrm{~Hz}, 3 \mathrm{H}) .{ }^{13} \mathbf{C}$ NMR $\left(100 \mathrm{MHz}, \mathrm{CDCl}_{3}\right) \delta 166.8,162.4$, $145.4,142.1,132.3,130.0,127.4,123.5,120.3,114.3,52.3,30.8,30.2,30.2,23.5$, 22.6, 14.6. HRMS (ESI) m/z: $[\mathrm{M}+\mathrm{H}]^{+}$Calcd. for $\mathrm{C}_{18} \mathrm{H}_{24} \mathrm{NO}_{3} 302.1751$; Found 302.1752 .

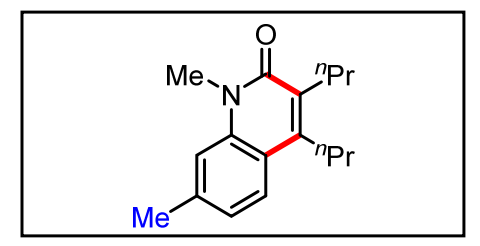

\section{1,7-Dimethyl-3,4-dipropylquinolin-2(1H)-one (3q)}

White solid (77.2 mg, 60\%), m.p. $105-107^{\circ} \mathrm{C} .{ }^{1} \mathbf{H}$ NMR $\left(400 \mathrm{MHz}, \mathrm{CDCl}_{3}\right) \delta 7.61(\mathrm{~d}, J$ $=8.4 \mathrm{~Hz}, 1 \mathrm{H}), 7.15(\mathrm{~s}, 1 \mathrm{H}), 7.05(\mathrm{~d}, J=8.2 \mathrm{~Hz}, 1 \mathrm{H}), 3.72(\mathrm{~s}, 3 \mathrm{H}), 2.87-2.79(\mathrm{~m}, 2 \mathrm{H})$, $2.73-2.64(\mathrm{~m}, 2 \mathrm{H}), 2.49(\mathrm{~s}, 3 \mathrm{H}), 1.69-1.51(\mathrm{~m}, 4 \mathrm{H}), 1.09(\mathrm{t}, J=7.4 \mathrm{~Hz}, 3 \mathrm{H}), 1.03(\mathrm{t}$, $J=7.4 \mathrm{~Hz}, 3 \mathrm{H}) .{ }^{13} \mathrm{C}$ NMR $\left(100 \mathrm{MHz}, \mathrm{CDCl}_{3}\right) \delta 162.5,145.1,139.5,139.0,130.3$, 125.0, 123.2, 118.5, 114.7, 31.0, 30.1, 29.8, 23.5, 22.8, 22.0, 14.7, 14.7. HRMS (ESI) $\mathrm{m} / \mathrm{z}:[\mathrm{M}+\mathrm{H}]^{+}$Calcd. for $\mathrm{C}_{17} \mathrm{H}_{24} \mathrm{NO} 258.1852$; Found 258.1856.

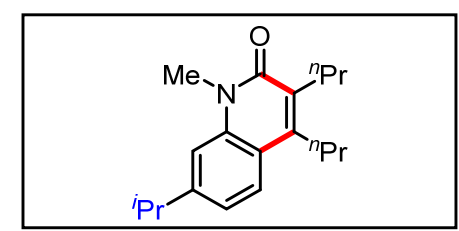

7-Isopropyl-1-methyl-3,4-dipropylquinolin-2(1H)-one (3r) 
Colorless oil (121.2 mg, 85\%). ${ }^{1} \mathbf{H}$ NMR $\left(400 \mathrm{MHz}, \mathrm{CDCl}_{3}\right) \delta 7.66(\mathrm{~d}, J=8.4 \mathrm{~Hz}, 1 \mathrm{H})$, $7.17(\mathrm{~s}, 1 \mathrm{H}), 7.12(\mathrm{~d}, J=8.0 \mathrm{~Hz}, 1 \mathrm{H}), 3.74(\mathrm{~s}, 3 \mathrm{H}), 3.08-2.97(\mathrm{~m}, 1 \mathrm{H}), 2.88-2.79(\mathrm{~m}$, $2 \mathrm{H}), 2.73-2.64(\mathrm{~m}, 2 \mathrm{H}), 1.69-1.61(\mathrm{~m}, 2 \mathrm{H}), 1.60-1.51(\mathrm{~m}, 2 \mathrm{H}), 1.31(\mathrm{~d}, J=6.8 \mathrm{~Hz}$, 6H), 1.09 (t, $J=7.4 \mathrm{~Hz}, 3 \mathrm{H}), 1.02(\mathrm{t}, J=7.4 \mathrm{~Hz}, 3 \mathrm{H}) .{ }^{13} \mathbf{C ~ N M R}\left(100 \mathrm{MHz}, \mathrm{CDCl}_{3}\right) \delta$ $162.5,150.5,145.1,139.0,130.4,125.2,120.4,118.9,112.1,34.6,31.0,30.1,29.8$, 24.1, 23.5, 22.7, 14.7, 14.7. HRMS (ESI) m/z: $[\mathrm{M}+\mathrm{H}]^{+}$Calcd. for $\mathrm{C}_{19} \mathrm{H}_{28} \mathrm{NO} 286.2165$; Found 286.2169.

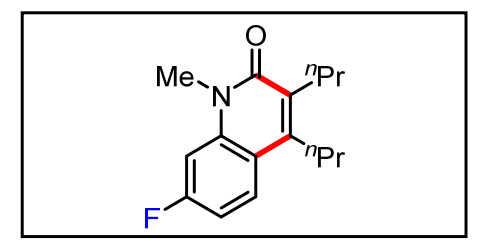

\section{7-Fluoro-1-methyl-3,4-dipropylquinolin-2(1H)-one (3s)}

White solid (107.1 mg, 82\%), m.p. 103-105 ${ }^{\circ} \mathrm{C} .{ }^{\mathbf{1}} \mathbf{H}$ NMR $\left(400 \mathrm{MHz}, \mathrm{CDCl}_{3}\right) \delta 7.69$ (dd, $J=9.2,6.4 \mathrm{~Hz}, 1 \mathrm{H}), 7.03(\mathrm{dd}, J=11.2,2.8 \mathrm{~Hz}, 1 \mathrm{H}), 6.98-6.92(\mathrm{~m}, 1 \mathrm{H}), 3.68(\mathrm{~s}, 3 \mathrm{H})$, $2.85-2.79(\mathrm{~m}, 2 \mathrm{H}), 2.71-2.65(\mathrm{~m}, 2 \mathrm{H}), 1.69-1.61(\mathrm{~m}, 2 \mathrm{H}), 1.60-1.51(\mathrm{~m}, 2 \mathrm{H}), 1.09$ $(\mathrm{t}, J=7.4 \mathrm{~Hz}, 3 \mathrm{H}), 1.03(\mathrm{t}, J=7.4 \mathrm{~Hz}, 3 \mathrm{H}) .{ }^{13} \mathbf{C ~ N M R}\left(100 \mathrm{MHz}, \mathrm{CDCl}_{3}\right) \delta 163.0(\mathrm{~d}, J$ $=246 \mathrm{~Hz}), 162.4,144.7,140.4(\mathrm{~d}, J=10.8 \mathrm{~Hz}), 130.3(\mathrm{~d}, J=2.6 \mathrm{~Hz}), 127.0(\mathrm{~d}, \mathrm{~J}=10.0$ $\mathrm{Hz}), 117.3(\mathrm{~d}, J=2.0 \mathrm{~Hz}), 109.5(\mathrm{~d}, J=22.2 \mathrm{~Hz}), 101.1$ (d, $J=26.2 \mathrm{~Hz}), 31.0,30.0$, 23.4, 22.6, 14.6, 14.6. ${ }^{19}$ F NMR $\left(376 \mathrm{MHz}, \mathrm{CDCl}_{3}\right) \delta-110.0$. HRMS (ESI) m/z: $[\mathrm{M}+\mathrm{H}]^{+}$Calcd. for $\mathrm{C}_{16} \mathrm{H}_{21} \mathrm{FNO} 262.1602$; Found 262.1601.

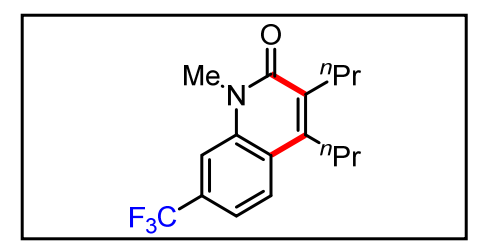

\section{1-Methyl-3,4-dipropyl-7-(trifluoromethyl)quinolin-2(1H)-one (3t)}

White solid (101.1 mg, 65\%), m.p. $101-103^{\circ} \mathrm{C} .{ }^{1} \mathbf{H}$ NMR (400 MHz, $\left.\mathrm{CDCl}_{3}\right) \delta 7.82(\mathrm{~d}$, $J=8.4 \mathrm{~Hz}, 1 \mathrm{H}), 7.56(\mathrm{~s}, 1 \mathrm{H}), 7.45(\mathrm{~d}, J=8.4 \mathrm{~Hz}, 1 \mathrm{H}), 3.76(\mathrm{~s}, 3 \mathrm{H}), 2.91-2.82(\mathrm{~m}, 2 \mathrm{H})$, $2.76-2.68(\mathrm{~m}, 2 \mathrm{H}), 1.70-1.61(\mathrm{~m}, 2 \mathrm{H}), 1.60-1.52(\mathrm{~m}, 2 \mathrm{H}), 1.10(\mathrm{t}, J=7.4 \mathrm{~Hz}, 3 \mathrm{H})$, $1.04(\mathrm{t}, J=7.4 \mathrm{~Hz}, 3 \mathrm{H}) .{ }^{13} \mathrm{C}$ NMR $\left(100 \mathrm{MHz}, \mathrm{CDCl}_{3}\right) \delta 162.0,144.3,138.8,133.9$, 130.7 (q, $J=32.4 \mathrm{~Hz}), 125.8,124.0(\mathrm{q}, J=270 \mathrm{~Hz}), 123.1,118.0$ (q, $J=3.0 \mathrm{~Hz}), 111.3$ $(\mathrm{q}, J=3.8 \mathrm{~Hz}), 30.9,30.3,30.0,23.3,22.6,14.5 .{ }^{19} \mathbf{F ~ N M R}\left(376 \mathrm{MHz}, \mathrm{CDCl}_{3}\right) \delta-62.3$. HRMS (ESI) m/z: [M+H] $]^{+}$Calcd. for $\mathrm{C}_{17} \mathrm{H}_{21} \mathrm{~F}_{3} \mathrm{NO} 312.1570$; Found 312.1572 .

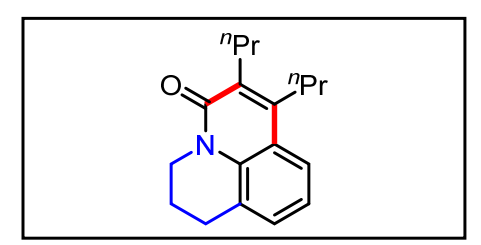

6,7-Dipropyl-2,3-dihydro-1 H,5H-pyrido[3,2,1-ij]quinolin-5-one (3u) ${ }^{10}$

White solid (113.1 mg, 84\%), m.p. 55-56 ${ }^{\circ} \mathrm{C} .{ }^{1} \mathbf{H}$ NMR $\left(400 \mathrm{MHz}, \mathrm{CDCl}_{3}\right) \delta 7.56(\mathrm{~d}, J$ $=8.0 \mathrm{~Hz}, 1 \mathrm{H}), 7.22(\mathrm{~d}, J=7.2 \mathrm{~Hz}, 1 \mathrm{H}), 7.11(\mathrm{t}, J=7.6 \mathrm{~Hz}, 1 \mathrm{H}), 4.21(\mathrm{t}, J=5.8 \mathrm{~Hz}, 2 \mathrm{H})$, $2.96(\mathrm{t}, J=6.2 \mathrm{~Hz}, 2 \mathrm{H}), 2.87-2.80(\mathrm{~m}, 2 \mathrm{H}), 2.73-2.67(\mathrm{~m}, 2 \mathrm{H}), 2.13-2.04(\mathrm{~m}, 2 \mathrm{H})$, $1.69-1.52(\mathrm{~m}, 4 \mathrm{H}), 1.09$ (t, $J=7.4 \mathrm{~Hz}, 3 \mathrm{H}), 1.03$ (t, $J=7.4 \mathrm{~Hz}, 3 \mathrm{H}) .{ }^{13} \mathbf{C}$ NMR (100 
$\left.\mathrm{MHz}, \mathrm{CDCl}_{3}\right) \delta 161.9,145.1,135.6,131.1,128.6,124.9,123.1,121.3,120.6,42.7$, $31.1,30.0,28.2,23.4,22.7,20.9,14.7,14.7$.

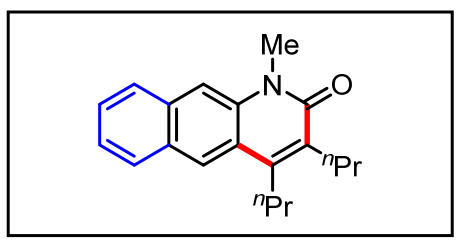

\section{1-Methyl-3,4-dipropylbenzo[g]quinolin-2(1H)-one (3v)}

White solid (142.2 mg, 97\%), m.p. 98-100 ${ }^{\circ} \mathrm{C} .{ }^{1} \mathbf{H}$ NMR (400 MHz, $\left.\mathrm{CDCl}_{3}\right) \delta 8.20$ (s, $1 \mathrm{H}), 7.93(\mathrm{~d}, J=8.2 \mathrm{~Hz}, 1 \mathrm{H}), 7.88(\mathrm{~d}, J=8.2 \mathrm{~Hz}, 1 \mathrm{H}), 7.66(\mathrm{~s}, 1 \mathrm{H}), 7.52(\mathrm{t}, J=7.6 \mathrm{~Hz}$, $1 \mathrm{H}), 7.43(\mathrm{t}, J=7.6 \mathrm{~Hz}, 1 \mathrm{H}), 3.82(\mathrm{~s}, 3 \mathrm{H}), 3.02-2.94(\mathrm{~m}, 2 \mathrm{H}), 2.78-2.69(\mathrm{~m}, 2 \mathrm{H})$, $1.80-1.69(\mathrm{~m}, 2 \mathrm{H}), 1.66-1.56(\mathrm{~m}, 2 \mathrm{H}), 1.16(\mathrm{t}, J=7.4 \mathrm{~Hz}, 3 \mathrm{H}), 1.06(\mathrm{t}, J=7.4 \mathrm{~Hz}$, $3 \mathrm{H}) .{ }^{13} \mathrm{C}$ NMR $\left(100 \mathrm{MHz}, \mathrm{CDCl}_{3}\right) \delta 162.5,144.6,137.0,133.5,131.9,128.8,128.3$, 127.2, 127.1, 124.7, 124.6, 121.4, 110.2, 31.1, 30.4, 30.0, 23.5, 22.9, 14.8, 14.7. HRMS (ESI) $\mathrm{m} / \mathrm{z}$ : $[\mathrm{M}+\mathrm{H}]^{+}$Calcd. for $\mathrm{C}_{20} \mathrm{H}_{24} \mathrm{NO} 294.1852$; Found 294.1858.

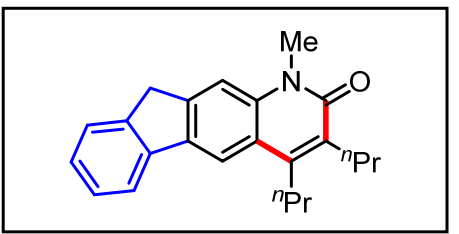

\section{1-Methyl-3,4-dipropyl-1,10-dihydro-2H-indeno[1,2-g]quinolin-2-one (3w)}

White solid (108.9 mg, 70\%), m.p. $141-143{ }^{\circ} \mathrm{C} .{ }^{1} \mathbf{H}$ NMR $\left(400 \mathrm{MHz}, \mathrm{CDCl}_{3}\right) \delta 8.05$ (s, $1 \mathrm{H}), 7.84(\mathrm{~d}, J=7.2 \mathrm{~Hz}, 1 \mathrm{H}), 7.55(\mathrm{~d}, J=7.2 \mathrm{~Hz}, 1 \mathrm{H}), 7.52(\mathrm{~s}, 1 \mathrm{H}), 7.41(\mathrm{t}, J=7.4 \mathrm{~Hz}$, 1H), $7.32(\mathrm{t}, J=7.4 \mathrm{~Hz}, 1 \mathrm{H}), 4.01(\mathrm{~s}, 2 \mathrm{H}), 3.80(\mathrm{~s}, 3 \mathrm{H}), 3.00-2.92(\mathrm{~m}, 2 \mathrm{H}), 2.78-2.70$ $(\mathrm{m}, 2 \mathrm{H}), 1.79-1.68(\mathrm{~m}, 2 \mathrm{H}), 1.66-1.55(\mathrm{~m}, 2 \mathrm{H}), 1.16(\mathrm{t}, J=7.4 \mathrm{~Hz}, 3 \mathrm{H}), 1.06(\mathrm{t}, J=$ $7.4 \mathrm{~Hz}, 3 \mathrm{H}) .{ }^{13} \mathbf{C}$ NMR $\left(100 \mathrm{MHz}, \mathrm{CDCl}_{3}\right) \delta 162.3,145.3,145.0,142.9,141.1,138.5$, 136.1, 130.7, 127.1, 126.8, 125.2, 119.9, 119.7, 115.4, 110.9, 37.1, 31.1, 30.3, 30.2, 23.5, 22.8, 14.8, 14.7. HRMS (ESI) m/z: $[\mathrm{M}+\mathrm{H}]^{+}$Calcd. for $\mathrm{C}_{23} \mathrm{H}_{26} \mathrm{NO} 332.2009$; Found 332.2011.

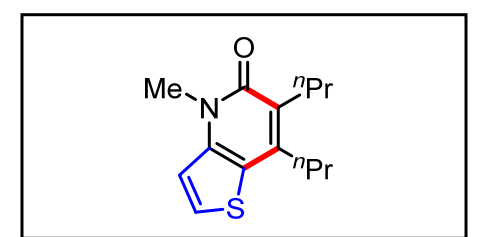

\section{4-Methyl-6,7-dipropylthieno[3,2-b]pyridin-5(4H)-one (3x)}

White solid (62.3 mg, 50\%), m.p. 96-98 ${ }^{\circ} \mathrm{C} .{ }^{1} \mathbf{H}$ NMR (400 MHz, $\left.\mathrm{CDCl}_{3}\right) \delta 7.45(\mathrm{~d}, J=$ $5.6 \mathrm{~Hz}, 1 \mathrm{H}), 7.03(\mathrm{~d}, J=5.6 \mathrm{~Hz}, 1 \mathrm{H}), 3.71(\mathrm{~s}, 3 \mathrm{H}), 2.73-2.67(\mathrm{~m}, 2 \mathrm{H}), 2.66-2.59(\mathrm{~m}$, $2 \mathrm{H}), 1.75-1.66(\mathrm{~m}, 2 \mathrm{H}), 1.61-1.52(\mathrm{~m}, 2 \mathrm{H}), 1.05(\mathrm{t}, J=7.4 \mathrm{~Hz}, 3 \mathrm{H}), 1.01(\mathrm{t}, J=7.4$ $\mathrm{Hz}, 3 \mathrm{H}) .{ }^{13} \mathbf{C}$ NMR $\left(100 \mathrm{MHz}, \mathrm{CDCl}_{3}\right) \delta 162.7,143.4,141.0,127.4,126.7,120.7$, 116.7, 34.6, 32.3, 29.9, 22.8, 22.6, 14.6, 14.6. HRMS (ESI) m/z: $[\mathrm{M}+\mathrm{H}]^{+}$Calcd. for $\mathrm{C}_{14} \mathrm{H}_{20} \mathrm{NOS} 250.1260$; Found 250.1263. 


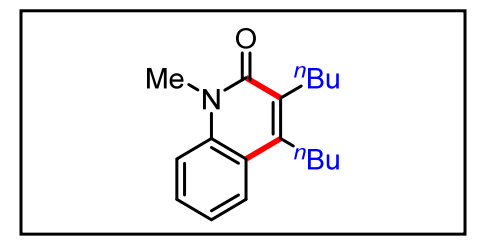

3,4-Dibutyl-1-methylquinolin-2(1H)-one (4a) ${ }^{10}$

White solid (120.7 mg, 89\%), m.p. $44-46{ }^{\circ} \mathrm{C} .{ }^{1} \mathbf{H}$ NMR (400 MHz, $\left.\mathrm{CDCl}_{3}\right) \delta 7.73$ (d, $J$ $=8.0 \mathrm{~Hz}, 1 \mathrm{H}), 7.49(\mathrm{t}, J=7.8 \mathrm{~Hz}, 1 \mathrm{H}), 7.34(\mathrm{~d}, J=8.4 \mathrm{~Hz}, 1 \mathrm{H}), 7.23(\mathrm{t}, J=7.6 \mathrm{~Hz}, 1 \mathrm{H})$, $3.74(\mathrm{~s}, 3 \mathrm{H}), 2.91-2.84(\mathrm{~m}, 2 \mathrm{H}), 2.75-2.70(\mathrm{~m}, 2 \mathrm{H}), 1.63-1.42(\mathrm{~m}, 8 \mathrm{H}), 0.99(\mathrm{t}, J=$ $6.8 \mathrm{~Hz}, 3 \mathrm{H}), 0.97$ (t, $J=6.8 \mathrm{~Hz}, 3 \mathrm{H}) .{ }^{13} \mathbf{C ~ N M R}\left(100 \mathrm{MHz}, \mathrm{CDCl}_{3}\right) \delta 162.3,145.1,138.9$, $131.5,129.1,125.0,121.8,120.8,114.3,32.2,31.6,29.8,28.6,27.9,23.4,23.3,14.1$, 14.0 .

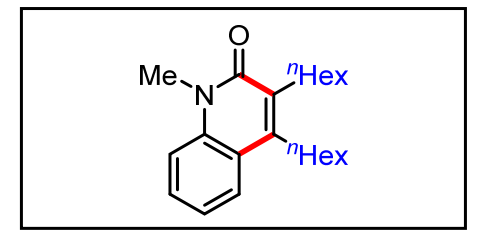

1,1'-(1-Methyl-2-oxo-1,2-dihydroquinoline-3,4-diyl)bis(hexan-1-one) (4b)

Colorless oil (144.0 mg, 88\%). ${ }^{1} \mathbf{H}$ NMR $\left(400 \mathrm{MHz}, \mathrm{CDCl}_{3}\right) \delta 7.74(\mathrm{~d}, J=8.0 \mathrm{~Hz}, 1 \mathrm{H})$, $7.50(\mathrm{t}, J=7.8 \mathrm{~Hz}, 1 \mathrm{H}), 7.35(\mathrm{~d}, J=8.4 \mathrm{~Hz}, 1 \mathrm{H}), 7.24(\mathrm{t}, J=7.8 \mathrm{~Hz}, 1 \mathrm{H}), 3.74(\mathrm{~s}, 3 \mathrm{H})$, $2.91-2.83(\mathrm{~m}, 2 \mathrm{H}), 2.78-2.66(\mathrm{~m}, 2 \mathrm{H}), 1.71-1.58(\mathrm{~m}, 3 \mathrm{H}), 1.56-1.42(\mathrm{~m}, 6 \mathrm{H}), 1.41$ $-1.28(\mathrm{~m}, 8 \mathrm{H}), 0.98-0.86(\mathrm{~m}, 6 \mathrm{H}) .{ }^{13} \mathbf{C} \mathbf{~ N M R}\left(100 \mathrm{MHz}, \mathrm{CDCl}_{3}\right) \delta 162.3,145.2$, 139.0, 131.6, 129.1, 125.0, 121.8, 120.8, 114.3, 31.8, 31.7, 30.1, 30.0, 29.9, 29.8, 29.4, 28.9, 28.2, 22.8, 22.7, 14.2, 14.2. HRMS (ESI) m/z: $[\mathrm{M}+\mathrm{H}]^{+}$Calcd. for $\mathrm{C}_{22} \mathrm{H}_{34} \mathrm{NO}$ 328.2635; Found 328.2637.

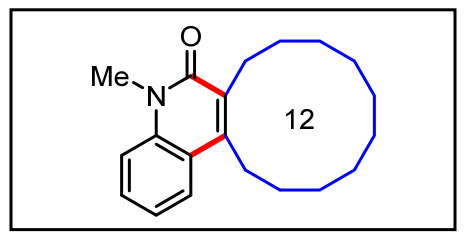

5-Methyl-7,8,9,10,11,12,13,14,15,16-decahydrocyclododeca[c]quinolin-6(5H)-one $(4 \mathrm{c})^{12}$

White solid (126.3 mg, 85\%), m.p. $171-173{ }^{\circ} \mathrm{C} .{ }^{1} \mathbf{H}$ NMR $\left(400 \mathrm{MHz}, \mathrm{CDCl}_{3}\right) \delta 7.78(\mathrm{~d}$, $J=8.0 \mathrm{~Hz}, 1 \mathrm{H}), 7.49(\mathrm{t}, J=7.8 \mathrm{~Hz}, 1 \mathrm{H}), 7.34(\mathrm{~d}, J=8.8 \mathrm{~Hz}, 1 \mathrm{H}), 7.23(\mathrm{t}, J=7.6 \mathrm{~Hz}$, $1 \mathrm{H}), 3.73(\mathrm{~s}, 3 \mathrm{H}), 3.01-2.92(\mathrm{~m}, 2 \mathrm{H}), 2.83-2.75(\mathrm{~m}, 2 \mathrm{H}), 1.84-1.71(\mathrm{~m}, 4 \mathrm{H}), 1.65-$ 1.44 (m, 12H). ${ }^{13} \mathbf{C}$ NMR $\left(100 \mathrm{MHz}, \mathrm{CDCl}_{3}\right) \delta 162.3,145.4,138.7,131.8,129.0,125.4$, $121.7,120.9,114.2,29.7,27.8,27.5,27.5,26.7,26.6,26.6,26.3,26.3,22.3,22.1$.

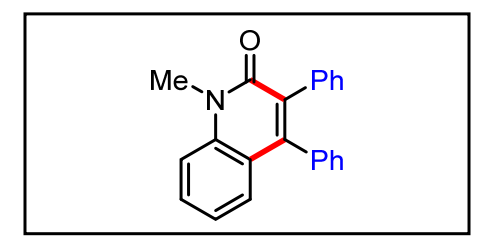

1-Methyl-3,4-diphenylquinolin-2(1H)-one (4d) ${ }^{10}$

L4 instead of $\mathbf{L 1 1}$ was used. White solid (38.9 mg, 25\%), m.p. 216-217 ${ }^{\circ} \mathrm{C}$. ${ }^{\mathbf{1}} \mathbf{H}$ NMR 
$\left(400 \mathrm{MHz}, \mathrm{CDCl}_{3}\right) \delta 7.56(\mathrm{t}, J=7.4 \mathrm{~Hz}, 1 \mathrm{H}), 7.44(\mathrm{~d}, J=8.4 \mathrm{~Hz}, 1 \mathrm{H}), 7.35-7.22(\mathrm{~m}$, 4H), $7.19-7.06(\mathrm{~m}, 8 \mathrm{H}), 3.84(\mathrm{~s}, 3 \mathrm{H}) .{ }^{13} \mathbf{C}$ NMR (100 MHz, $\left.\mathrm{CDCl}_{3}\right) \delta 161.9,147.8$, $139.7,136.5,136.1,132.2,130.7,130.4,130.0,128.6,128.1,127.6,127.6,127.0$, $122.0,121.6,114.2,30.2$.

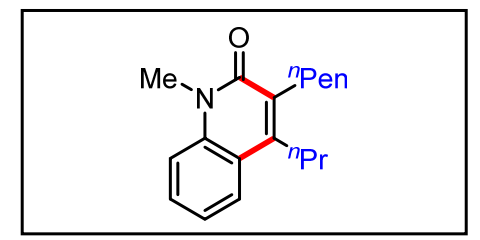

\section{1-Methyl-4-pentyl-3-propylquinolin-2(1H)-one (4e)}

White solid (40.7 mg, 30\%), m.p. 39-41 ${ }^{\circ} \mathrm{C} .{ }^{1} \mathbf{H}$ NMR (400 MHz, $\left.\mathrm{CDCl}_{3}\right) \delta 7.73(\mathrm{~d}, J=$ $8.0 \mathrm{~Hz}, 1 \mathrm{H}), 7.49$ (t, $J=7.8 \mathrm{~Hz}, 1 \mathrm{H}), 7.35(\mathrm{~d}, J=8.4 \mathrm{~Hz}, 1 \mathrm{H}), 7.24(\mathrm{t}, J=7.4 \mathrm{~Hz}, 1 \mathrm{H})$, $3.74(\mathrm{~s}, 3 \mathrm{H}), 2.92-2.82(\mathrm{~m}, 2 \mathrm{H}), 2.74-2.67(\mathrm{~m}, 2 \mathrm{H}), 1.70-1.61(\mathrm{~m}, 2 \mathrm{H}), 1.59-1.49$ (m, 2H), $1.48-1.32(\mathrm{~m}, 4 \mathrm{H}), 1.10(\mathrm{t}, J=6.2 \mathrm{~Hz}, 3 \mathrm{H}), 0.92(\mathrm{t}, J=6.6 \mathrm{~Hz}, 3 \mathrm{H}) .{ }^{13} \mathbf{C}$ NMR $\left(100 \mathrm{MHz}, \mathrm{CDCl}_{3}\right) \delta 162.4,145.0,139.0,131.8,129.2,125.1,121.9,120.8$, 114.4, 32.5, 31.0, 29.9, 29.2, 28.2, 23.5, 22.7, 14.8, 14.2. HRMS (ESI) m/z: $[\mathrm{M}+\mathrm{H}]^{+}$ Calcd. for $\mathrm{C}_{18} \mathrm{H}_{26} \mathrm{NO} 272.2009$; Found 272.2011.

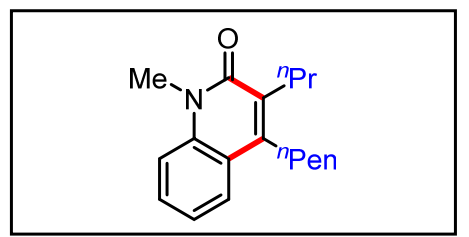

\section{1-Methyl-3-pentyl-4-propylquinolin-2(1H)-one (4e')}

Colorless oil (65.1 mg, 48\%). ${ }^{1} \mathbf{H}$ NMR $\left(400 \mathrm{MHz}, \mathrm{CDCl}_{3}\right) \delta 7.74(\mathrm{~d}, J=8.0 \mathrm{~Hz}, 1 \mathrm{H})$, 7.49 (t, $J=7.8 \mathrm{~Hz}, 1 \mathrm{H}), 7.35$ (d, $J=8.4 \mathrm{~Hz}, 1 \mathrm{H}), 7.24(\mathrm{t}, J=7.4 \mathrm{~Hz}, 1 \mathrm{H}), 3.74(\mathrm{~s}, 3 \mathrm{H})$, $2.92-2.82(\mathrm{~m}, 2 \mathrm{H}), 2.74-2.67(\mathrm{~m}, 2 \mathrm{H}), 1.66-1.55(\mathrm{~m}, 4 \mathrm{H}), 1.54-1.45(\mathrm{~m}, 2 \mathrm{H}), 1.44$ $-1.35(\mathrm{~m}, 2 \mathrm{H}), 1.04(\mathrm{t}, J=6.8 \mathrm{~Hz}, 3 \mathrm{H}), 0.94(\mathrm{t}, J=6.6 \mathrm{~Hz}, 3 \mathrm{H}) .{ }^{13} \mathbf{C}$ NMR $(100 \mathrm{MHz}$, $\left.\mathrm{CDCl}_{3}\right) \delta 162.3,145.4,138.9,131.3,129.2,125.1,121.9,120.8,114.4,32.5,30.2,29.9$, 29.8, 28.9, 22.7, 22.6, 14.7, 14.2. HRMS (ESI) m/z: $[\mathrm{M}+\mathrm{H}]^{+}$Calcd. for $\mathrm{C}_{18} \mathrm{H}_{26} \mathrm{NO}$ 272.2009; Found 272.2011.

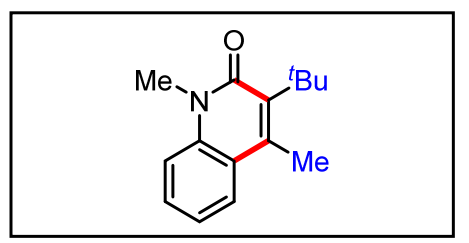

\section{3-(tert-Butyl)-1,4-dimethylquinolin-2(1H)-one (4f)}

White solid (34.4 mg, 30\%), m.p. $78-80{ }^{\circ} \mathrm{C} .{ }^{1} \mathbf{H}$ NMR (400 MHz, $\left.\mathrm{CDCl}_{3}\right) \delta 7.76(\mathrm{~d}, J=$ $8.0 \mathrm{~Hz}, 1 \mathrm{H}), 7.46(\mathrm{t}, J=7.8,1 \mathrm{H}), 7.26(\mathrm{~d}, J=8.4 \mathrm{~Hz}, 1 \mathrm{H}), 7.19$ (t, $J=7.6 \mathrm{~Hz}, 1 \mathrm{H}), 3.67$ (s, 3H), $2.61(\mathrm{~s}, 3 \mathrm{H}), 1.56(\mathrm{~s}, 9 \mathrm{H}) .{ }^{13} \mathbf{C}$ NMR $\left(100 \mathrm{MHz}, \mathrm{CDCl}_{3}\right) \delta 162.3,140.8,138.7$, 138.2, 129.0, 124.8, 123.2, 121.5, 113.6, 37.7, 31.7, 29.8, 19.1. HRMS (ESI) m/z: $[\mathrm{M}+\mathrm{H}]^{+}$Calcd. for $\mathrm{C}_{15} \mathrm{H}_{20} \mathrm{NO} 230.1539$; Found 230.1538 . 


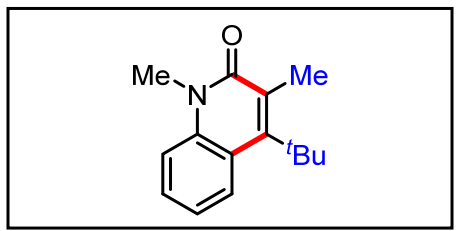

4-(tert-Butyl)-1,3-dimethylquinolin-2(1H)-one (4f')

White solid (43.5 mg, 38\%), m.p. 82-84 ${ }^{\circ} \mathrm{C} .{ }^{1} \mathbf{H}$ NMR (400 MHz, $\left.\mathrm{CDCl}_{3}\right) \delta 8.05(\mathrm{~d}, J=$ $7.4 \mathrm{~Hz}, 1 \mathrm{H}), 7.39$ (t, $J=7.8 \mathrm{~Hz}, 1 \mathrm{H}), 7.31(\mathrm{~d}, J=8.4 \mathrm{~Hz}, 1 \mathrm{H}), 7.13(\mathrm{t}, J=7.8 \mathrm{~Hz}, 1 \mathrm{H})$, $3.69(\mathrm{~s}, 3 \mathrm{H}), 2.41(\mathrm{~s}, 3 \mathrm{H}), 1.62(\mathrm{~s}, 9 \mathrm{H}) .{ }^{13} \mathrm{C} \mathbf{N M R}\left(100 \mathrm{MHz}, \mathrm{CDCl}_{3}\right) \delta 163.8,153.0$, 138.1, 128.8, 127.9, 127.6, 121.7, 120.1, 114.3, 38.6, 33.0, 30.5, 18.1. HRMS (ESI) $\mathrm{m} / \mathrm{z}:[\mathrm{M}+\mathrm{H}]^{+}$Calcd. for $\mathrm{C}_{15} \mathrm{H}_{20} \mathrm{NO} 230.1539$; Found 230.1538 .

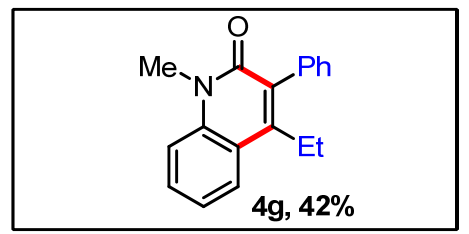

\section{4-Ethyl-1-methyl-3-phenylquinolin-2(1H)-one (4g)}

White solid (55.3 mg, 42\%), m.p. 136-138 ${ }^{\circ} \mathrm{C} .{ }^{1} \mathbf{H}$ NMR (400 MHz, $\left.\mathrm{CDCl}_{3}\right) \delta 7.74$ (d, $J$ $=8.0 \mathrm{~Hz}, 1 \mathrm{H}), 7.48(\mathrm{t}, J=7.8 \mathrm{~Hz}, 1 \mathrm{H}), 7.34(\mathrm{q}, J=7.2 \mathrm{~Hz}, 3 \mathrm{H}), 7.28(\mathrm{~d}, J=6.8 \mathrm{~Hz}, 1 \mathrm{H})$, $7.21-7.13(\mathrm{~m}, 3 \mathrm{H}), 3.66$ (s, 3H), 2.62 (q, $J=7.4 \mathrm{~Hz}, 2 \mathrm{H}), 1.08(\mathrm{t}, J=7.4 \mathrm{~Hz}, 3 \mathrm{H}) .{ }^{13} \mathbf{C}$ NMR $\left(100 \mathrm{MHz}, \mathrm{CDCl}_{3}\right) \delta 162.0,148.3,139.8,137.0,132.1,130.1,129.6,128.5$, 127.5, 125.8, 122.1, 120.2, 114.7, 29.9, 23.1, 14.7. HRMS (ESI) m/z: $[\mathrm{M}+\mathrm{H}]^{+}$Calcd. for $\mathrm{C}_{18} \mathrm{H}_{28} \mathrm{NO} 264.1383$; Found 264.1385.

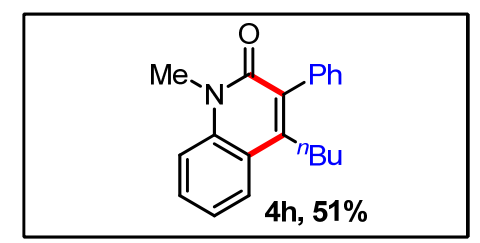

4-Butyl-1-methyl-3-phenylquinolin-2(1H)-one (4h) $)^{12}$

White solid (74.2 mg, 51\%), m.p. 84-86 ${ }^{\circ} \mathrm{C} .{ }^{1} \mathbf{H}$ NMR (400 MHz, $\left.\mathrm{CDCl}_{3}\right) \delta 7.72(\mathrm{~d}, J=$ $8.4 \mathrm{~Hz}, 1 \mathrm{H}), 7.48(\mathrm{t}, J=7.8 \mathrm{~Hz}, 1 \mathrm{H}), 7.34(\mathrm{q}, J=8.0 \mathrm{~Hz}, 3 \mathrm{H}), 7.28(\mathrm{~d}, J=7.2 \mathrm{~Hz}, 1 \mathrm{H})$, $7.22-7.13(\mathrm{~m}, 3 \mathrm{H}), 3.67(\mathrm{~s}, 3 \mathrm{H}), 2.66-2.44(\mathrm{~m}, 2 \mathrm{H}), 1.52-1.39(\mathrm{~m}, 2 \mathrm{H}), 1.26-1.12$ $(\mathrm{m}, 2 \mathrm{H}), 0.71(\mathrm{t}, J=7.4 \mathrm{~Hz}, 3 \mathrm{H}) .{ }^{13} \mathbf{C} \mathbf{~ N M R}\left(100 \mathrm{MHz}, \mathrm{CDCl}_{3}\right) \delta 161.9,147.1,139.6$, 137.0, 132.3, 130.1, 129.7, 128.3, 127.4, 125.8, 122.0, 120.5, 114.6, 32.4, 29.9, 29.6, 23.0, 13.7 .

\section{Crystal Structure}

\section{Crystal Structure Information of $\mathbf{4 h}$}

$4 \mathrm{~mL}$ of DCM was added to a $10 \mathrm{~mL}$ oven-dried glass sample bottle with $70 \mathrm{mg}$ pure $4 \mathrm{~h}$ to dissolve the sample, sealed with perforated paper, and then the solvent was slowly dried at room temperature to obtain crystals. Single crystal X-ray diffraction data were collected on Rigaku Saturn70 diffractometer. The crystal structure has been deposited at the Cambridge Crystallographic Data Centre (CCDC): 1887875 
Compound Name: 4-Butyl-1-methyl-3-phenylquinolin-2(1H)-one

Formula: $\mathrm{C}_{20} \mathrm{H}_{21} \mathrm{NO}$

Unit Cell Parameters: a 9.0557(18) b 9.4682(19) c 10.491(2) P 1 (2)

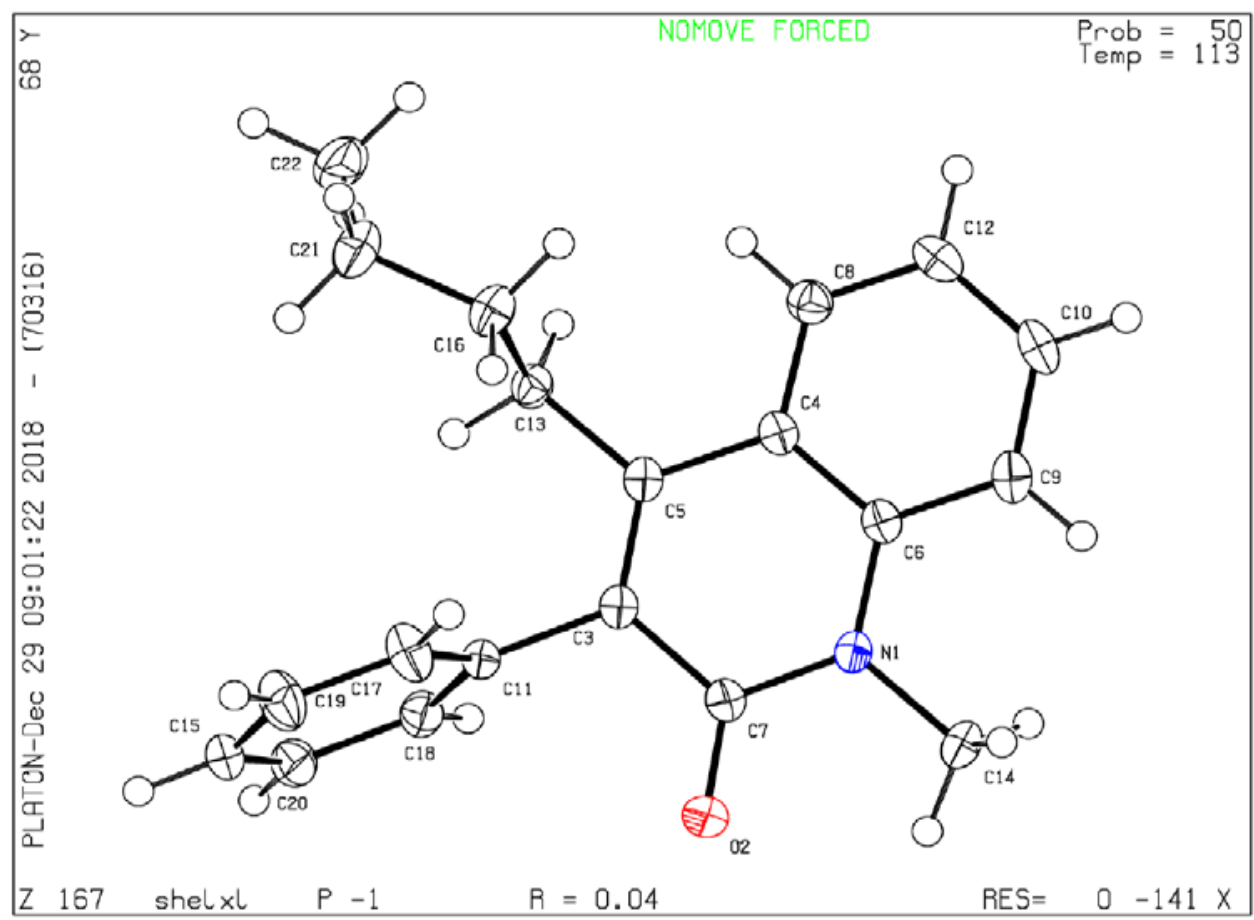

The ellipsoid contour percent probability level is $30 \%$ 。

\section{Mechanistic Experiments}

\subsection{Deuterated-substrate preparation}

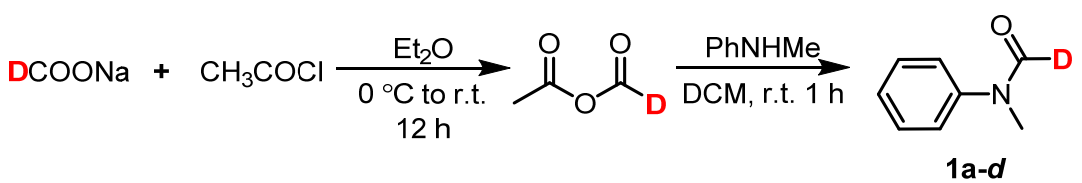

\section{$N$-Methyl- $N$-phenylformamide-d (1a-d)}

To the solution of sodium formate- $d(99 \% \mathrm{D}, 0.83 \mathrm{~g}, 12.0 \mathrm{mmol})$ in dry $\mathrm{Et}_{2} \mathrm{O}(5.0 \mathrm{~mL})$ was added acetyl chloride $(0.71 \mathrm{~mL}, 10.0 \mathrm{mmol})$ slowly $\left(<25^{\circ} \mathrm{C}\right)$ under $\mathrm{N}_{2}$ atmosphere. The mixture was stirred at room temperature for overnight. Filtered the solid and the filtrate was evaporated under reduced pressure at low temperature to obtain the acetic formic anhydride- $d(0.89 \mathrm{~g}, 100 \%)$. The obtained acetic formic anhydride- $d(0.89 \mathrm{~g}, 10$ mmol) was added to the solution of $N$-methyl aniline $(0.54 \mathrm{~g}, 5.0 \mathrm{mmol})$ in $\mathrm{CH}_{2} \mathrm{Cl}_{2}(10$ $\mathrm{mL}$ ), and the mixture was stirred at r.t. for one hour. The reaction was quenched with 5 $\mathrm{mL}$ of saturated aqueous $\mathrm{NaHCO}_{3}$, and the aqueous layer was extracted with $\mathrm{CH}_{2} \mathrm{Cl}_{2}(2$ $\times 10 \mathrm{~mL})$. The combined organic fractions were washed with brine $(10 \mathrm{~mL})$, dried over anhydrous $\mathrm{Na}_{2} \mathrm{SO}_{4}$, filtered, and concentrated in vacuo. The residue was purified by column chromatography ( $25 \%$ EtOAc/n-hexane) to afford the title compound as a colorless oil $(0.54 \mathrm{~g}, 79 \%)$. ${ }^{1} \mathbf{H}$ NMR $\left(400 \mathrm{MHz}, \mathrm{CDCl}_{3}\right) \delta 7.42(\mathrm{t}, J=7.6 \mathrm{~Hz}, 2 \mathrm{H}), 7.28$ $(\mathrm{t}, J=7.8 \mathrm{~Hz}, 1 \mathrm{H}), 7.18(\mathrm{~d}, J=8.2 \mathrm{~Hz}, 2 \mathrm{H}), 3.33(\mathrm{~s}, 3 \mathrm{H})$. 

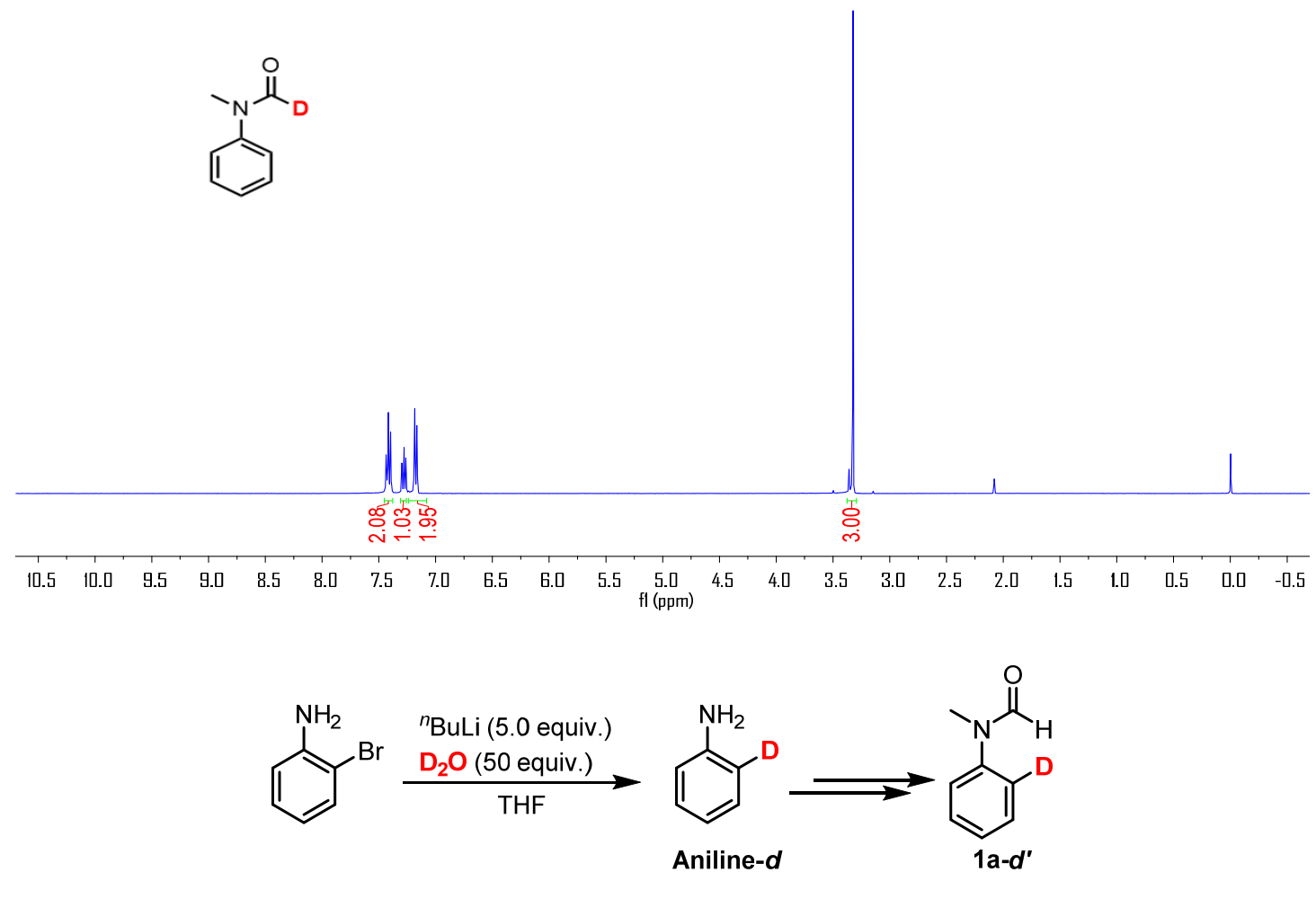

\section{$N$-Methyl- $N$-(phenyl-2-d)formamide (1a-d')}

The deuterated substrate was prepared according to the reported procedure in literature. ${ }^{13}{ }^{1} \mathbf{H}$ NMR $\left(400 \mathrm{MHz}, \mathrm{CDCl}_{3}\right) \delta 8.48(\mathrm{~s}, 1 \mathrm{H}), 7.42(\mathrm{t}, J=7.6 \mathrm{~Hz}, 2 \mathrm{H}), 7.28(\mathrm{t}$, $J=7.8 \mathrm{~Hz}, 1 \mathrm{H}), 7.18(\mathrm{~d}, J=8.2 \mathrm{~Hz}, 1 \mathrm{H}), 3.33(\mathrm{~s}, 3 \mathrm{H})$.
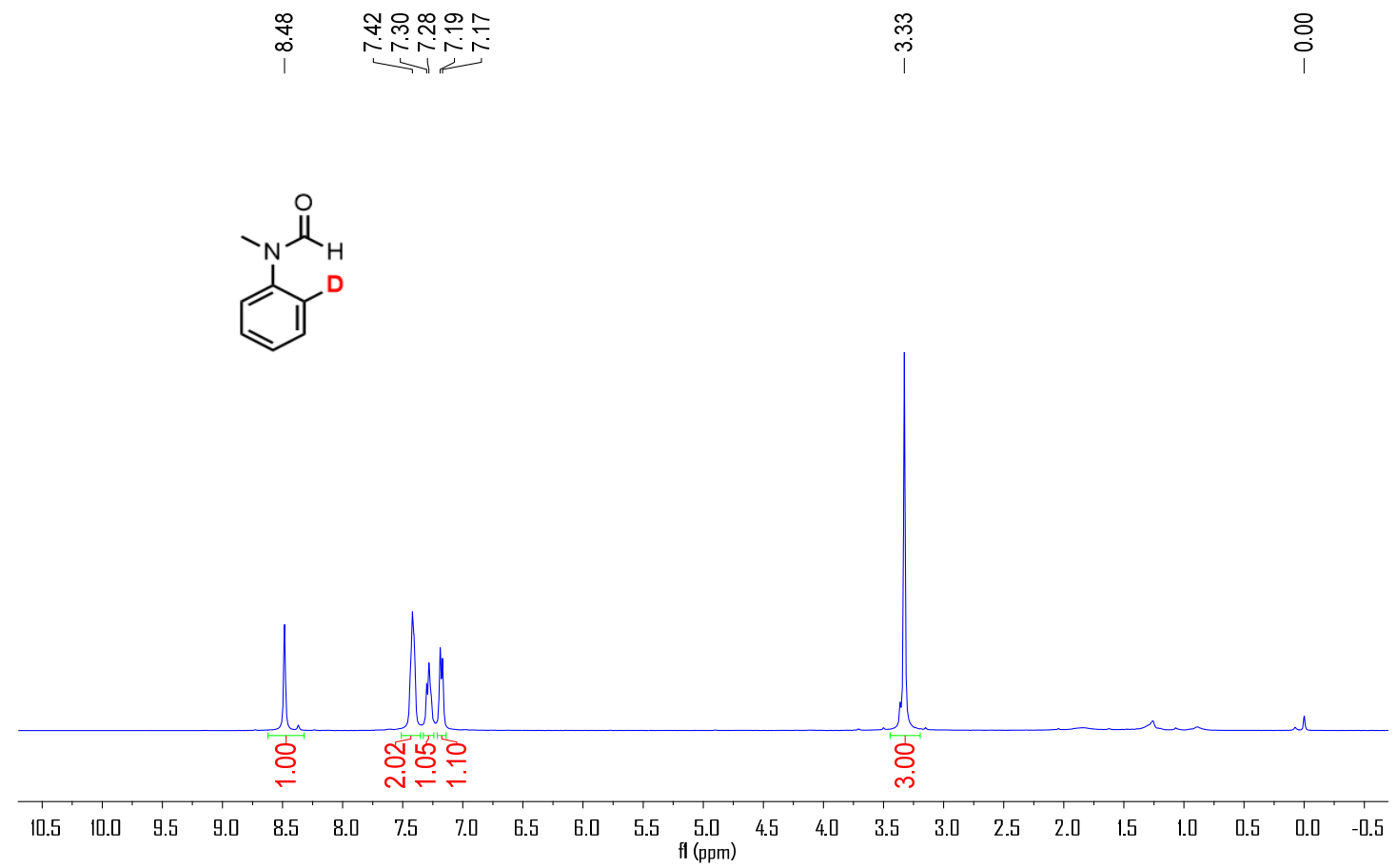


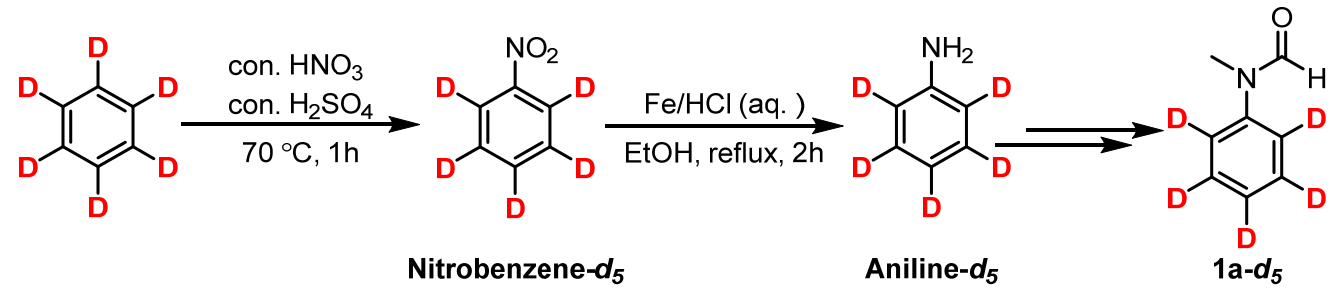

\section{$N$-Methyl- $N$-(phenyl- $\left.d_{5}\right)$ formamide (1a- $\left.d_{5}\right)$}

The deuterated substrate was prepared according to the reported procedure in literature. ${ }^{14}{ }^{1} \mathbf{H}$ NMR $\left(400 \mathrm{MHz}, \mathrm{CDCl}_{3}\right) \delta 8.48(\mathrm{~s}, 1 \mathrm{H}), 3.32(\mathrm{~s}, 3 \mathrm{H})$.<smiles>[2H]c1c([2H])c([2H])c(N(C)C=O)c([2H])c1[2H]</smiles><smiles>[Li][V]</smiles>

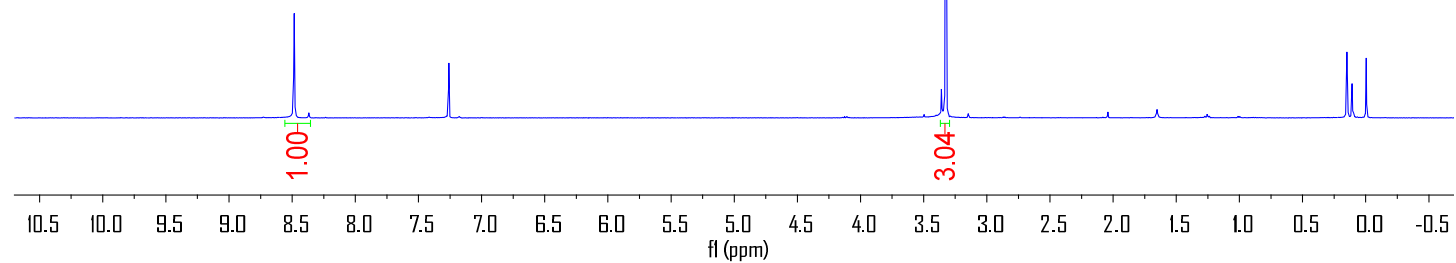

\subsection{Deuterium-Labeling Experiment}<smiles>[2H]C(=O)N(C)c1ccccc1</smiles>

1a-d

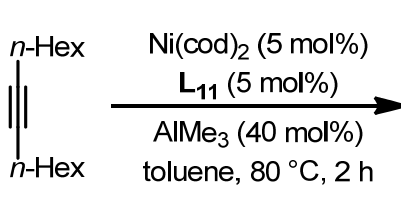

2b

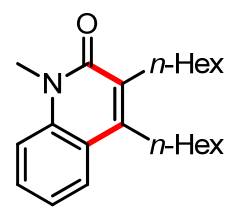

4b, $92 \%$

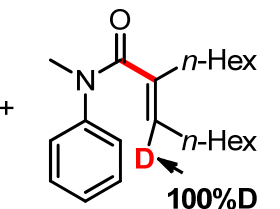

$5 b-d, 8 \%$

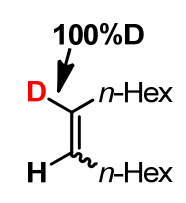

6b-d, $89 \%$<smiles>[2H]c1c([2H])c([2H])c(N(C)C=O)c([2H])c1[2H]</smiles><smiles>[Mg][Ca][Ca]</smiles>

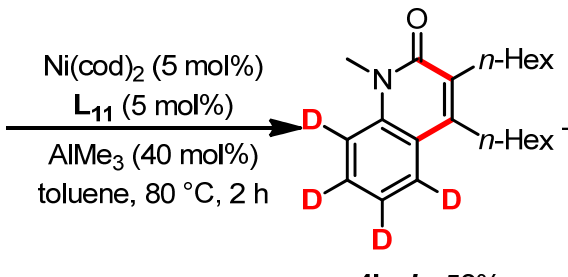

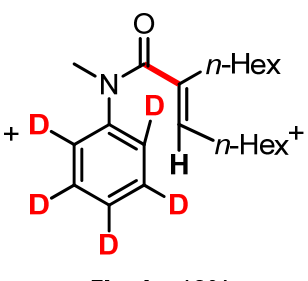

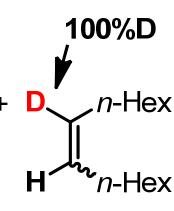

$4 \mathrm{~b}-\mathrm{d}_{4}, 50 \%$

$6 \mathrm{~b}-\mathrm{d}, 50 \%$

The experiments were carried out according to the typical procedure, and the results were detected by GC-MS. 


\subsection{Parallel Reactions for KIE Determination of Carbonylic C-H of Formamide}

Parallel reactions were set up following the general procedure at $50{ }^{\circ} \mathrm{C}$ by using $1 \mathrm{a}$ and 1a-d as substrate respectively. Aliquots were taken at 2.5 minute intervals for the first ten minutes. Product yield was determined by ${ }^{1} \mathrm{H} \mathrm{NMR}$ using $\mathrm{CH}_{2} \mathrm{Br}_{2}$ as an internal standard. Data points represent the average of two runs.<smiles>CN(C=O)c1ccccc1</smiles>

$1 \mathrm{a}, 0.2 \mathrm{mmol}$<smiles>CN(C(=O)I)c1ccccc1</smiles>

1a-d, $0.2 \mathrm{mmol}$

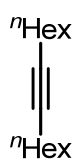

2a, $0.4 \mathrm{mmol}$

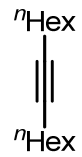

2a, $0.4 \mathrm{mmol}$

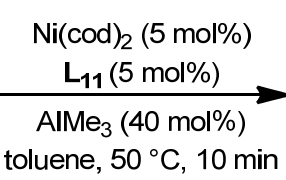

toluene, $50^{\circ} \mathrm{C}, 10 \mathrm{~min}$

$\mathrm{Ni}(\operatorname{cod})_{2}(5 \mathrm{~mol} \%)$

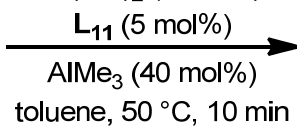

toluene, $50^{\circ} \mathrm{C}, 10 \mathrm{~min}$<smiles>[X]c1c(Cc2ccccc2)c(=O)n(C)c2ccccc12</smiles>

3a<smiles>[Y]c1cc2ccccc2n(C)c1=O</smiles>

$3 a$

$\mathrm{KIE}=k_{\mathrm{H}} / k_{\mathrm{D}}=1.86 / 1.74=1.07$

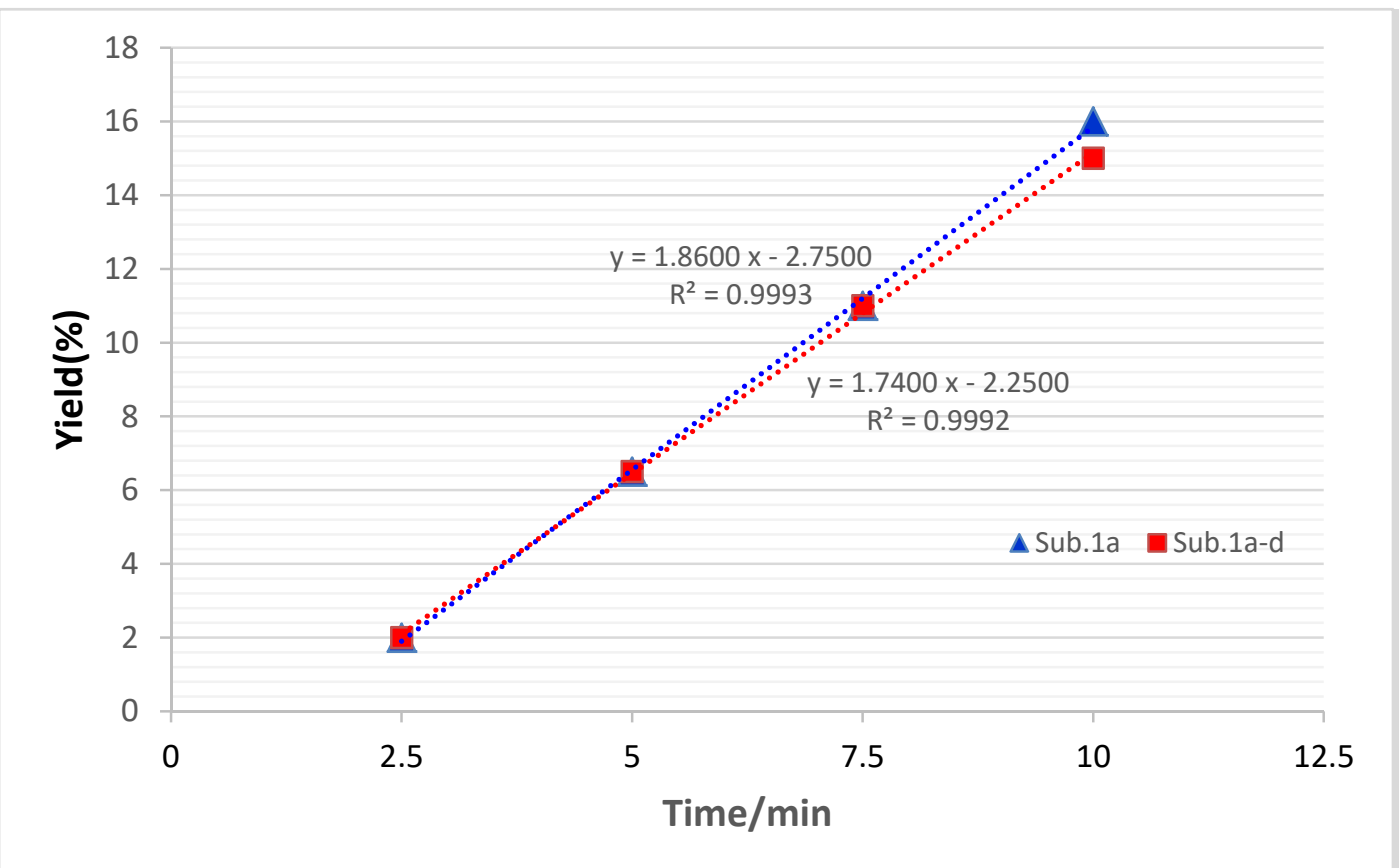

\subsection{Intramolecular Competitive Reaction}<smiles>CC(=O)N(C)c1ccccc1[OH2+]</smiles>

1a-d'

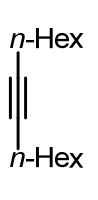

$2 b$

b

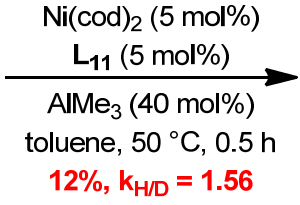<smiles>Cn1c(=O)c(C=[Te])c(O)c2ccccc21</smiles>

$4 b$

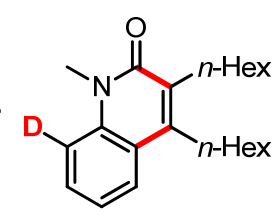

$4 b-d^{\prime}$ 
To a $15 \mathrm{~mL}$ oven dried tube were added SPO ligand $\mathbf{L}_{11}(2.2 \mathrm{mg}, 5 \mathrm{~mol} \%)$, $\mathrm{Ni}(\mathrm{cod})_{2}(2.8 \mathrm{mg}, 5 \mathrm{~mol} \%)$, dry degassed toluene $(0.1 \mathrm{~mL}), \mathbf{1 a - d}$ ( $\left.27.2 \mathrm{mg}, 0.2 \mathrm{mmol}\right)$, $\mathrm{AlMe}_{3}(80 \mu \mathrm{L}, 40 \mathrm{~mol} \%)$ and tetradec-7-yne $(77.7 \mathrm{mg}, 0.4 \mathrm{mmol})$ sequentially in an $\mathrm{N}_{2}$-filled glove-box. The tube was sealed and removed out of the glove-box. After heated at $50{ }^{\circ} \mathrm{C}$ in a dry block heater for $0.5 \mathrm{~h}$, the mixture was cooled to r.t. and quenched with $0.1 \mathrm{~mL}$ of 5\% EDTA disodium salt solution, then filtered through a short plug of silica gel (EtOAc as the eluent) and concentrated in vacuo. Further purification by flash column chromatography on silica gel (eluting with EtOAc $/ n$-hexane $=8 / 1)$ gave the pure product $\mathbf{4 b}$ and $\mathbf{4 b}-\boldsymbol{d}^{\prime}$ in $12 \%$ yield. $k_{\mathrm{H}} / k_{\mathrm{D}}=1.56$ was obtained by calculation of the ratio of two products.
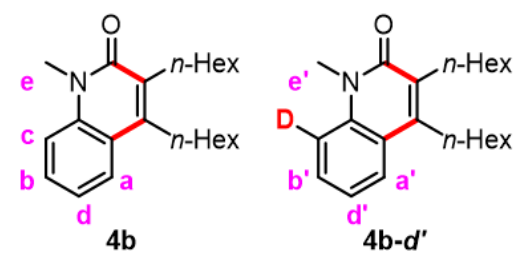

Calculation:

$\left(\mathbf{4 b}-d^{\prime}\right) /(\mathbf{4 b})=(1-0.39) / 0.39=1.56 / 1$ $\mathrm{KIE}=1.56$
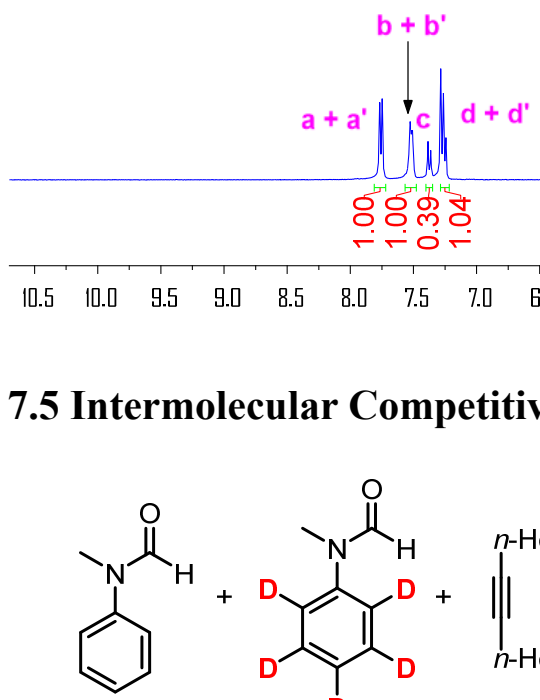

\section{o. of}

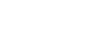

\author{
.
}

.


vacuo. Further purification by flash column chromatography on silica gel (eluting with EtOAc $/ n$-hexane $=8 / 1)$ gave the pure product $\mathbf{4 b}$ and $\mathbf{4 b}-\boldsymbol{d}_{\mathbf{4}}$ in $25 \%$ yield. $k_{\mathrm{H}} / k_{\mathrm{D}}=$ 1.50 was obtained by calculation of the ratio of two products.
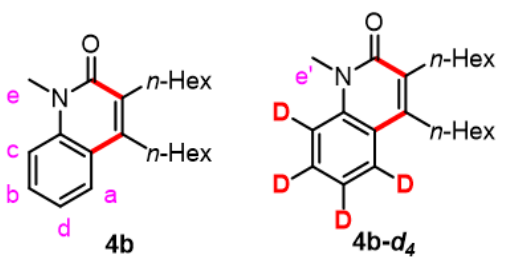

Calculation:

$(4 \mathbf{b}) /\left(\mathbf{4 b}-d_{4}\right)=0.6 /(1.0-0.6)=1.5 / 1$

$\mathrm{KIE}=1.50$

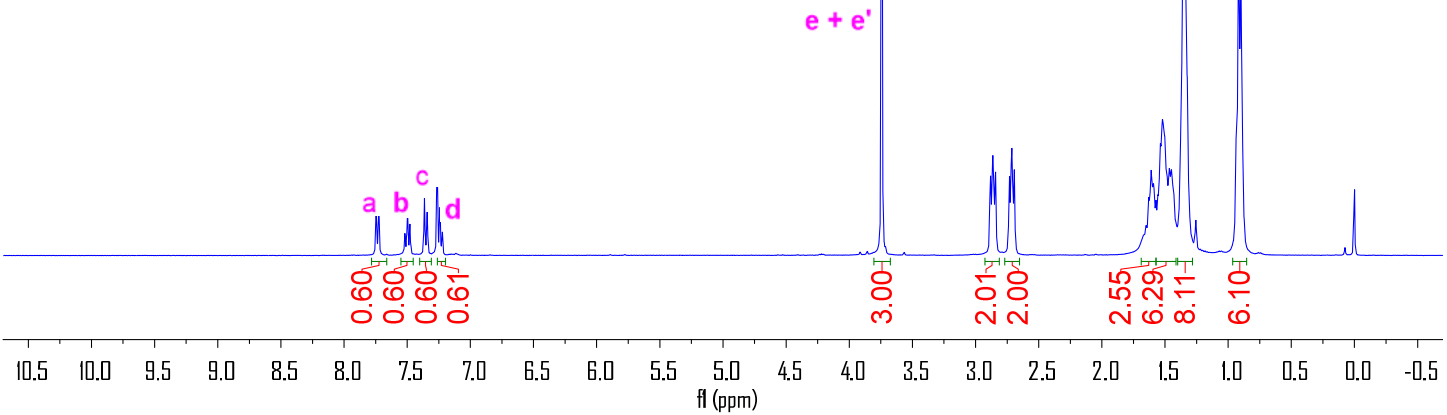

\section{6 ${ }^{31}$ P NMR Experiments}

\section{1) Detection of SPO-Al Complex}

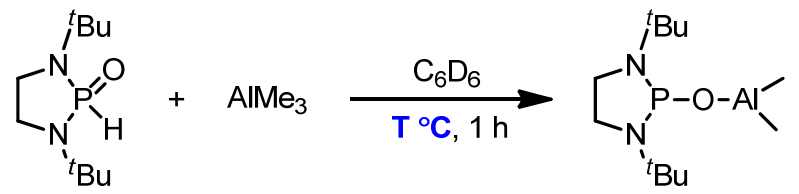

\begin{tabular}{cccc} 
L11, 0.1 $\mathbf{~ m m o l ~}$ & \multicolumn{2}{c}{ 0.1 $\mathbf{~ m o l}$} & complex A \\
\hline entry & $\mathrm{AlMe}_{3}$ & $\mathrm{~T}\left({ }^{\circ} \mathbf{C}\right)$ & ${ }^{31} \mathrm{P} \mathrm{NMR}(\delta$ ppm $)$ \\
\hline 1 & 0 & 25 & 6.86 \\
2 & 1.0 equiv & 80 & 7.05 \\
3 & 1.0 equiv & 120 & $\mathbf{8 8 . 0 4}$
\end{tabular}

To a $15 \mathrm{~mL}$ oven dried Schlenk tube were added $\mathbf{L}_{11}(21.8 \mathrm{mg}, 0.1 \mathrm{mmol}), \mathrm{C}_{6} \mathrm{D}_{6}$ $\left(0.5 \mathrm{~mL}\right.$, without TMS) and $\mathrm{AlMe}_{3}(100 \mu \mathrm{L}, 1.0 \mathrm{M}$ in hexane, $0.1 \mathrm{mmol})$ at room temperature under $\mathrm{N}_{2}$ atmosphere. After stirred for $1 \mathrm{~h}$ at specified temperature, the mixture was cooled to r.t., transferred into the NMR tube, sealed and monitored by ${ }^{31} \mathrm{P}$ NMR. ${ }^{31}$ P NMR (162 MHz, $\mathrm{C}_{6} \mathrm{D}_{6}$ without TMS) $\delta 88.04$. 


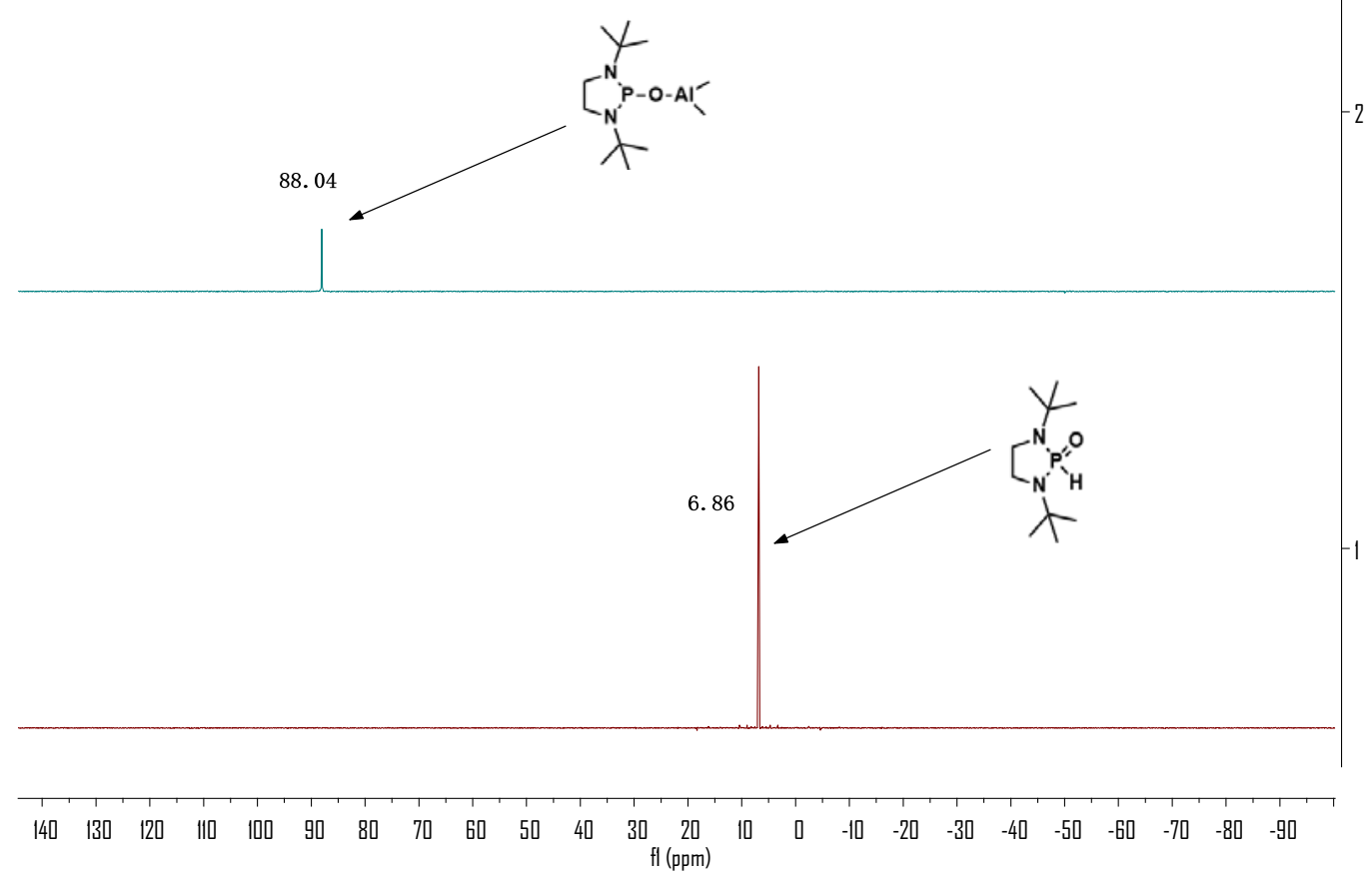

\section{2) Detection of Bimetallic Complex (SPO-Ni-AI)}

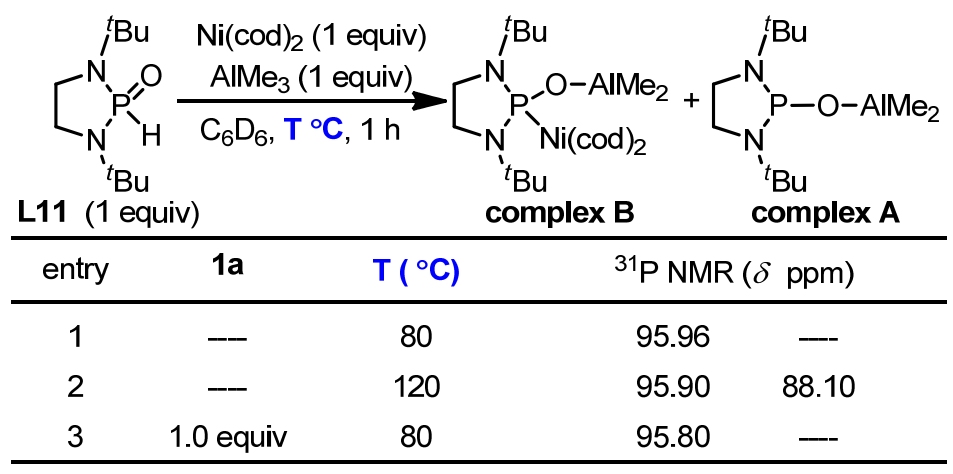

To a $15 \mathrm{~mL}$ oven dried Schlenk tube were added $\mathbf{L}_{\mathbf{1 1}}(21.8 \mathrm{mg}, 0.1 \mathrm{mmol})$, Ni(cod) (27.5 mg, $0.1 \mathrm{mmol}), \mathrm{C}_{6} \mathrm{D}_{6}(0.5 \mathrm{~mL}$, without TMS), $1 \mathrm{a}(13.5 \mathrm{mg}, 0.1 \mathrm{mmol}$, entry 3$)$, and $\mathrm{AlMe}_{3}(100 \mu \mathrm{L}, 1.0 \mathrm{M}$ in hexane, $0.1 \mathrm{mmol})$ at room temperature under $\mathrm{N}_{2}$ atmosphere. Cooled to r.t. After stirred for $1 \mathrm{~h}$ at specified temperature, the mixture was cooled to r.t., transferred into the NMR tube, sealed and monitored by ${ }^{31} \mathrm{P}$ NMR.

At $80^{\circ} \mathrm{C}$, only small amounts of complex $\mathbf{B}$ were formed. When heated to $120^{\circ} \mathrm{C}$ in a dry block heater, the amounts of complex $\mathbf{B}$ increased. Notably, the presence of substrate significantly promoted the formation of complex $\mathbf{B}$, probably owing the fact that the $\eta^{2}$-coordination of carbonyl of the formamide to $\mathrm{Ni}$ accelerated the isomeration of SPO ligand.

\section{Reactivity of Complex B}

The formed complex B-1a was subjected to alkyne at $80{ }^{\circ} \mathrm{C}$, and the desired product was obtained in $60 \%$ yield. 

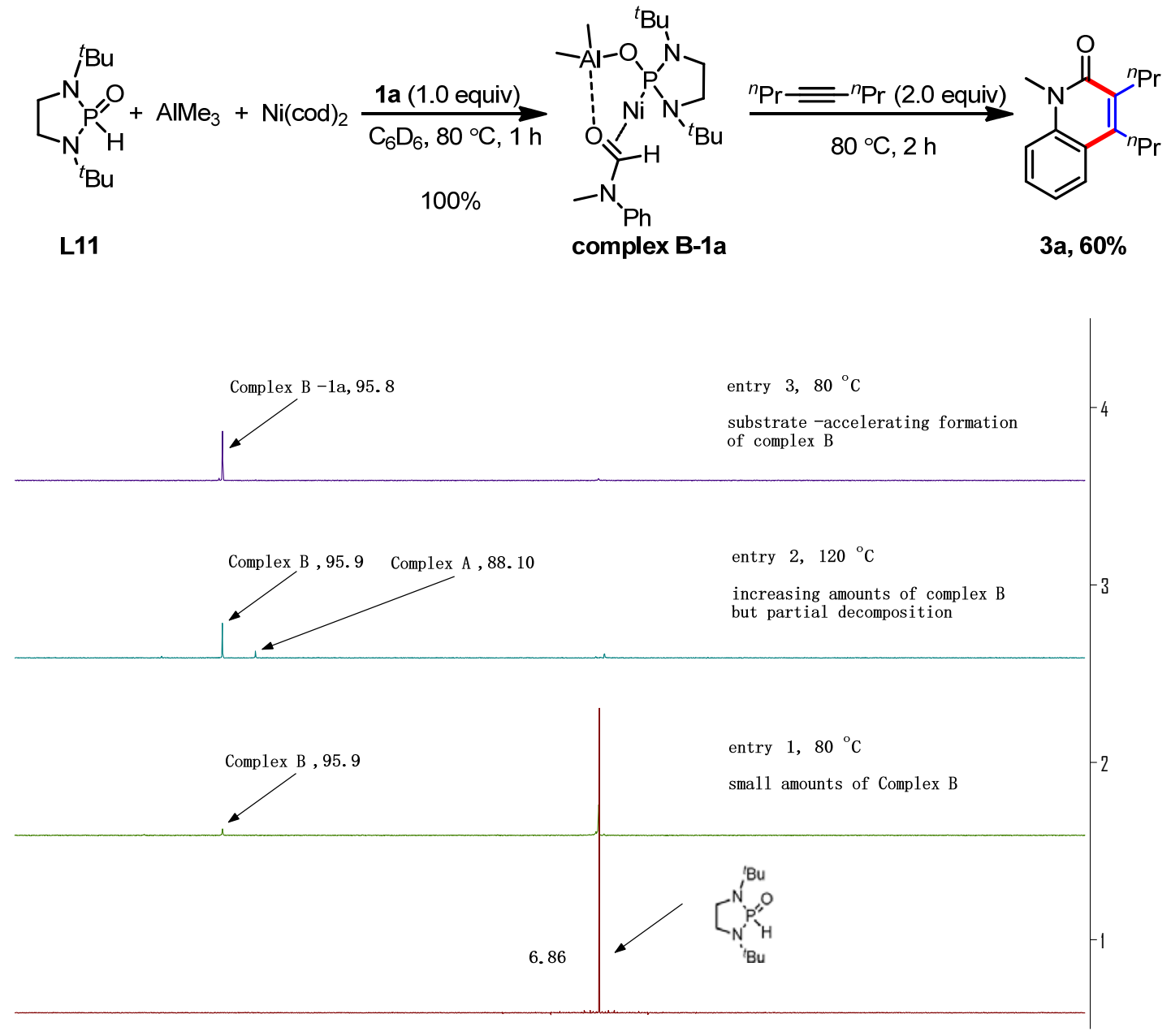

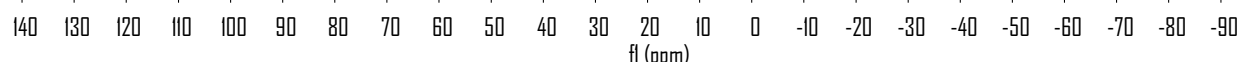




\section{Reference}

1. Li, X.-F..; Zhang, X.-G.; Chen, F.; Zhang, X.-H. J. Org. Chem. 2018, 83, 1281512821.

2. Yang, S.; Li, P.; Wang, Z.; Wang, L. Org. Lett. 2017, 19, 3386-3389.

3. Taneda, H.; Inamoto, K.; Kondo, Y. Chem. Commun. 2014, 50, 6523-6525.

4. Klapars, A.; Antilla, J. C.; Huang, X.; Buchwald, S. L. J. Am. Chem. Soc. 2001, 123, $7727-7729$.

5. Busacca, C. A.; Lorenz, J. C.; Grinberg, N.; Haddad, N.; Hrapchak, M.; Latli, B.; Lee, H.; Sabila, P.; Saha, A.; Sarvestani, M.; Shen, S.; Varsolona, R.; Wei, X.; Senanayake, C. H. Org. Lett. 2005, 7, 4277-4280.

6. Munoz, A.; Hubert, C.; Luche, J.-L. J. Org. Chem. 1996, 61, 6015- 6017.

7. Wang, Y.-X.; Qi, S.-L.; Luan, Y.-X.; Han, X.-W.; Wang, S.; Chen, H.; Ye, M. J. Am. Chem. Soc. 2018, 140, 5360-5364.

8. Ackermann, L.; Born, R. Angew. Chem., Int. Ed. 2005, 44, 2444-2447.

9. Thangavel, A.; Wieliczko, M.; Bacsa, J.; Scarborough, C. C. Inorg. Chem., 2013, $52,13282-13287$.

10. Zhu, F.; Li, Y.; Zhang, Z.; Wu, X.-F. Adv. Synth. Catal. 2016, 358, 3350-3354.

11. Nakai, K.; Kurahashi, T.; Matsubara, S. Org. Lett. 2013, 15, 856-859.

12. Li, X.; Li, X.; Jiao, N. J. Am. Chem. Soc. 2015, 137, 9246-9249.

13. Chi, Y.; Zhang, W.-X.; Xi, Z. Org. Lett. 2014, 16, 6274-6277.

14. Prakash, R.; Bora, B. R.; Boruah, R. C.; Gogoi, S. Org. Lett. 2018, 20, 2297-2300. 


\section{NMR Spectra}

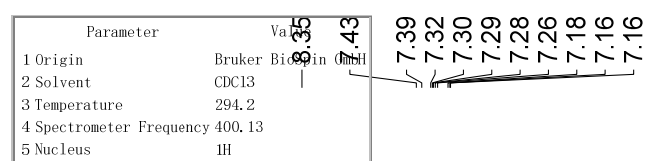

4 Spectrometer Frequency 400.

$1 \mathrm{~b}$

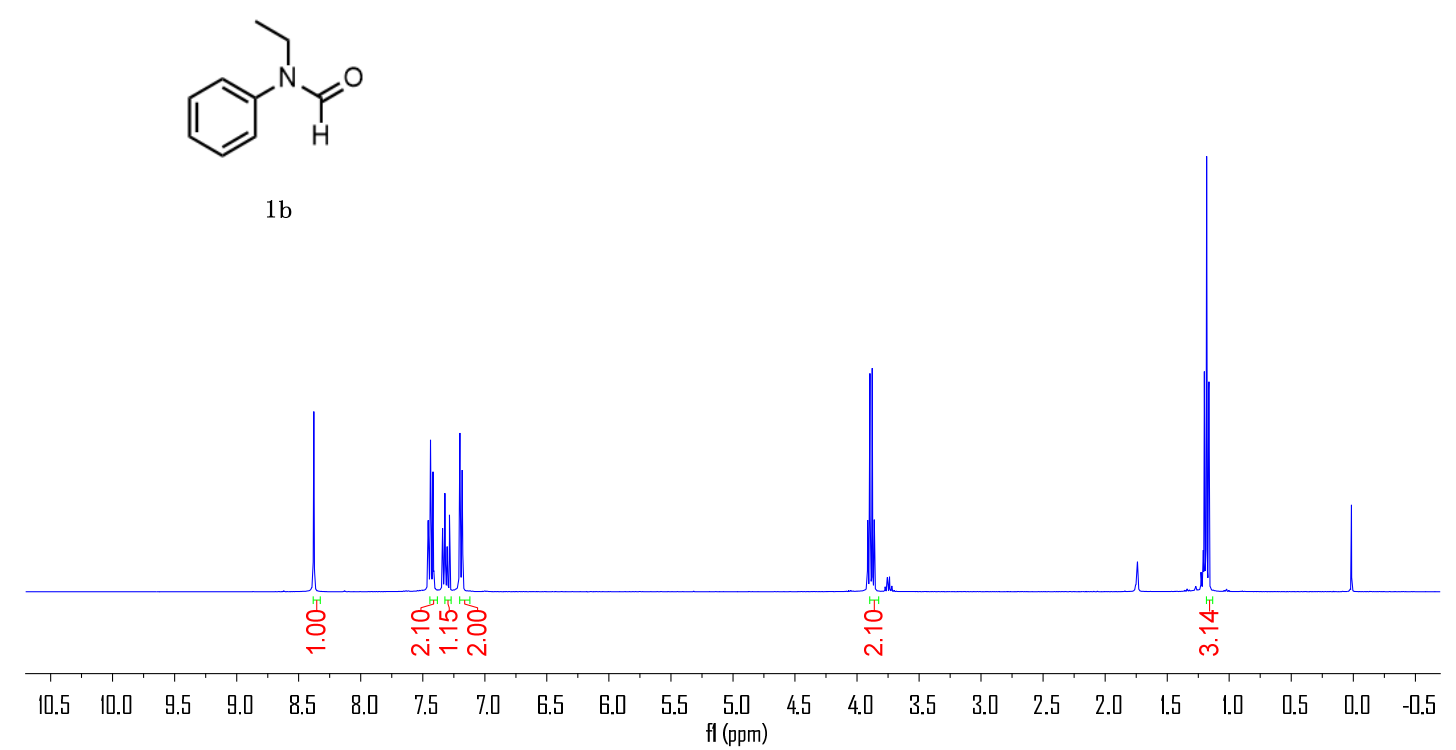

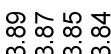

लं लंm

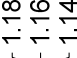

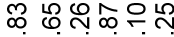

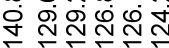

舟

NNo

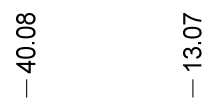

296.0

5 Nuclens<smiles>CCN(C=O)c1ccccc1</smiles>

$1 \mathrm{~b}$

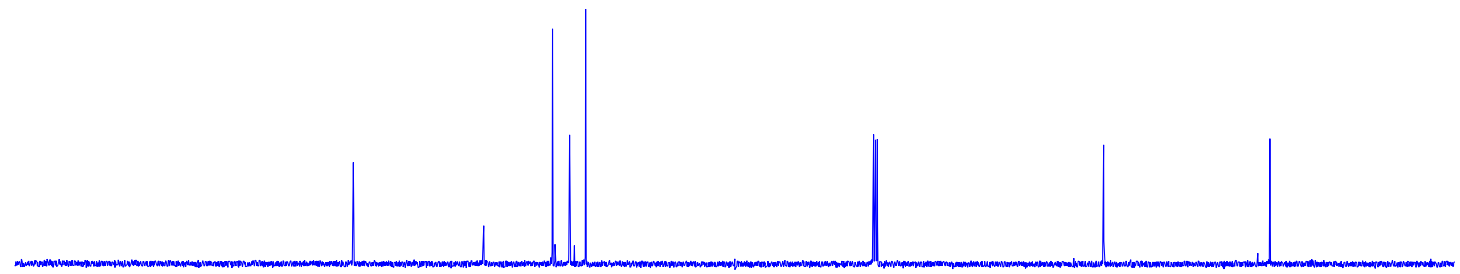

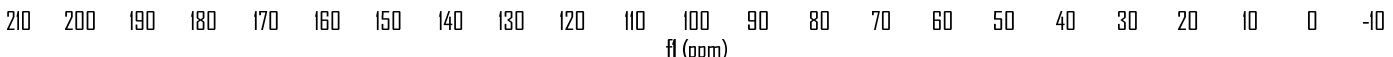




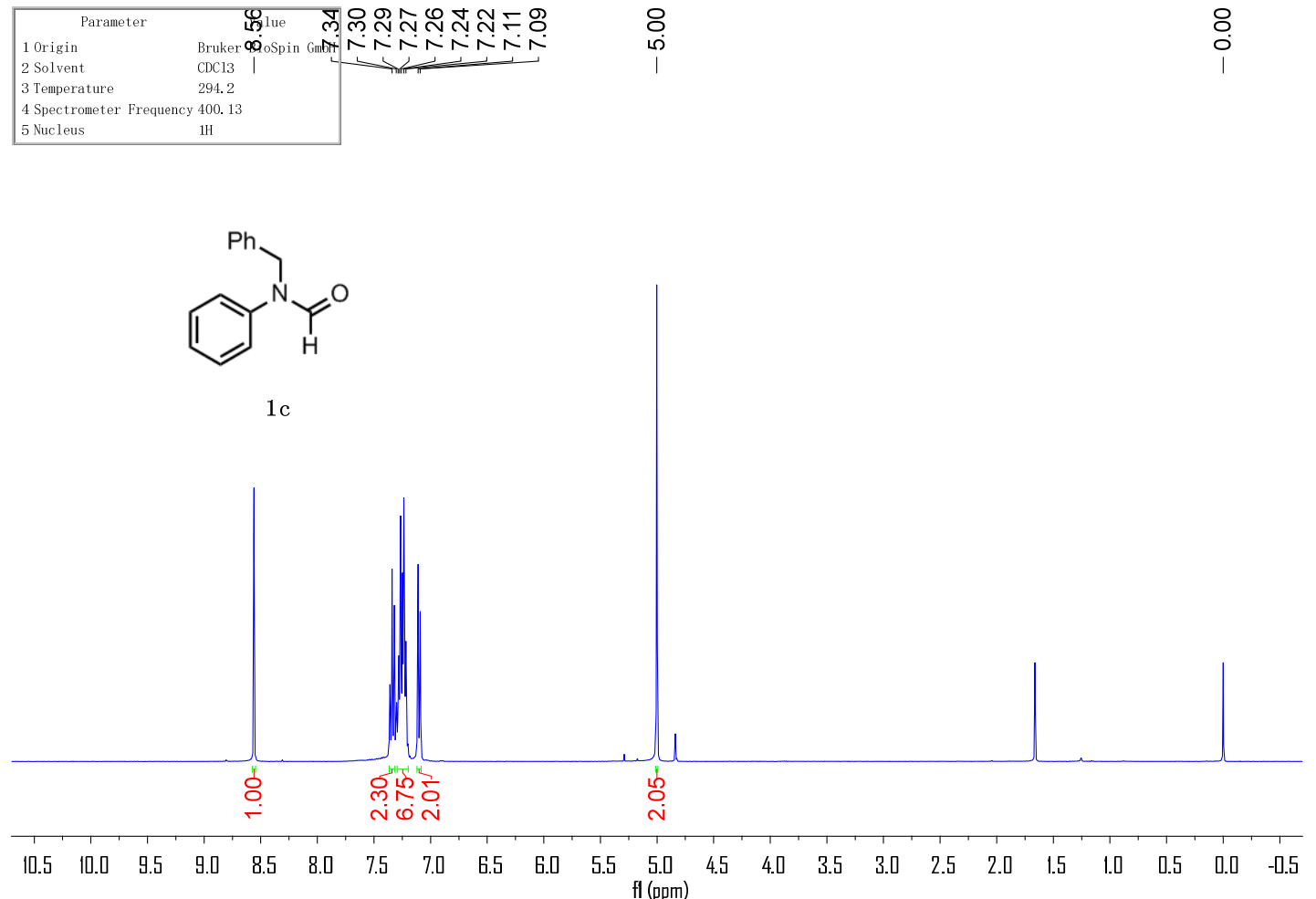

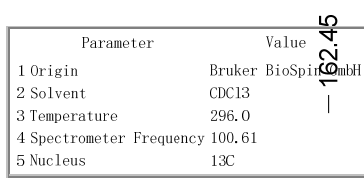

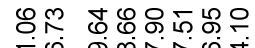

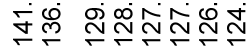

舟ํㅡㅁ

사요

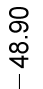<smiles>O=CC(=O)N(Cc1ccccc1)c1ccccc1</smiles>

$1 \mathrm{c}$

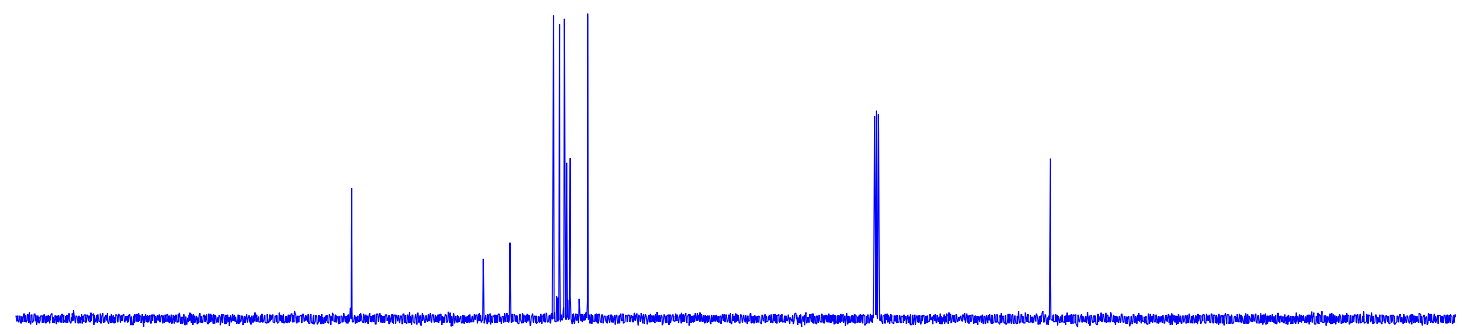

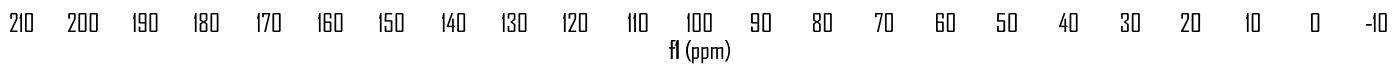




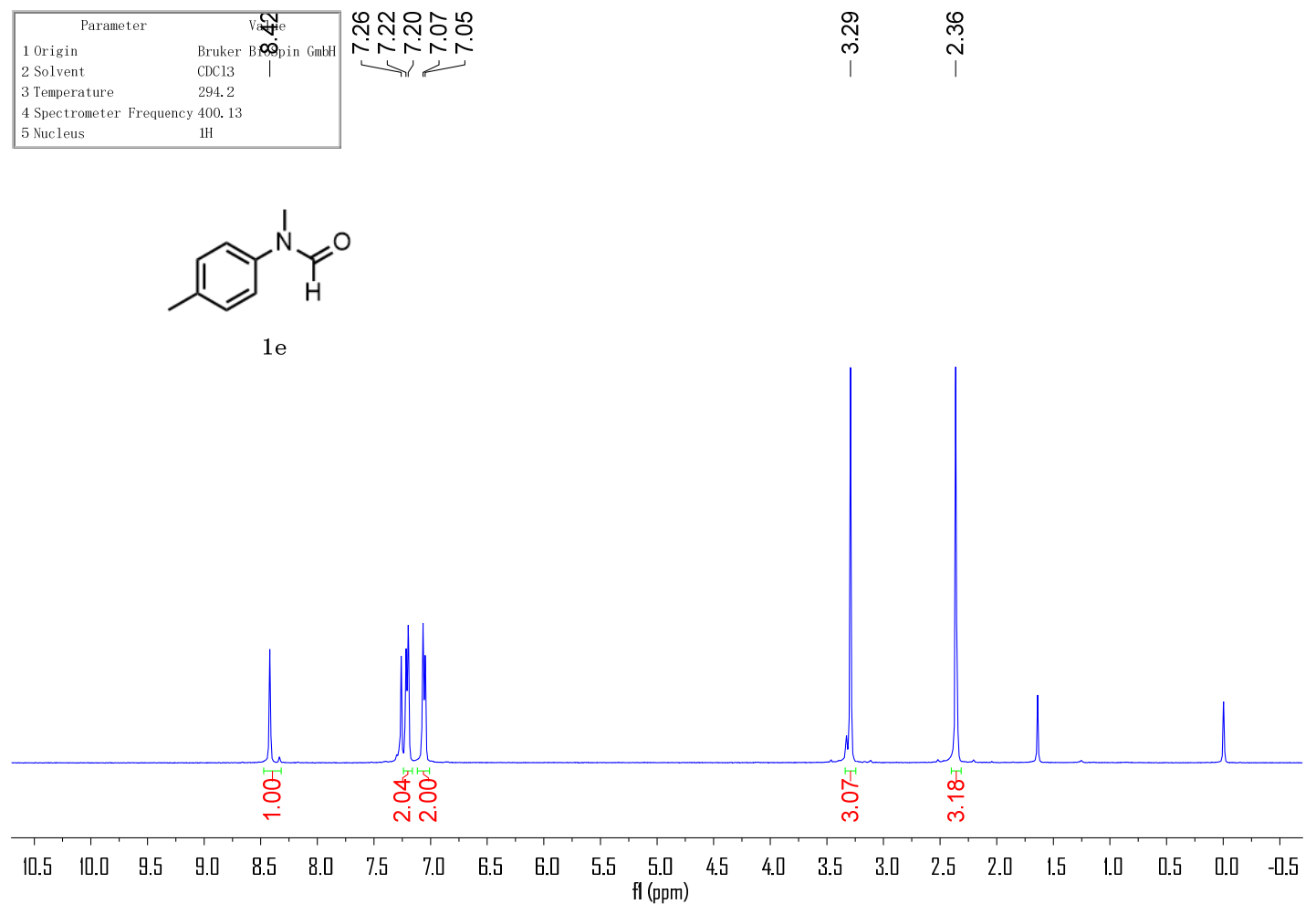

\begin{tabular}{|c|c|}
\hline Parameter & 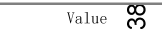 \\
\hline 1 Origin & Bruker BioSpin જึંib \\
\hline 2 Solvent & $\underline{\sigma}$ \\
\hline 3 Temperature & 296.0 \\
\hline 4 Spectrometer Frequenc & y 100.61 \\
\hline 5 Nucleus & $13 \mathrm{C}$ \\
\hline
\end{tabular}

융둥

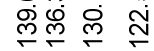

Кำ

5 Nucleus $\quad 13 \mathrm{C}$<smiles>Cc1ccc(N(C)C=O)cc1</smiles>

$1 \mathrm{e}$

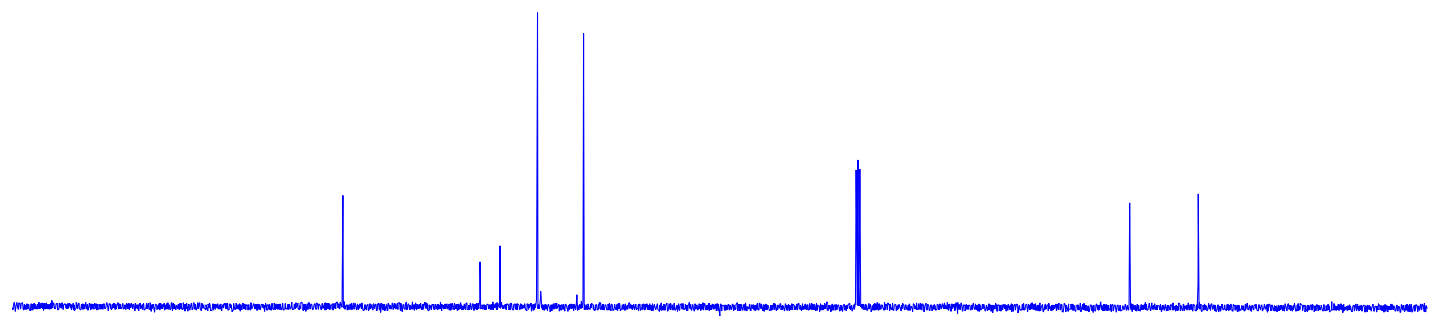

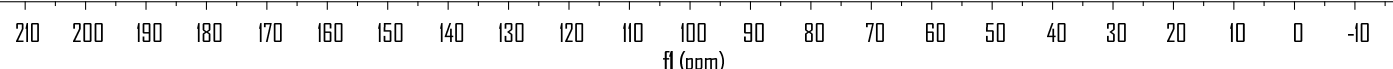




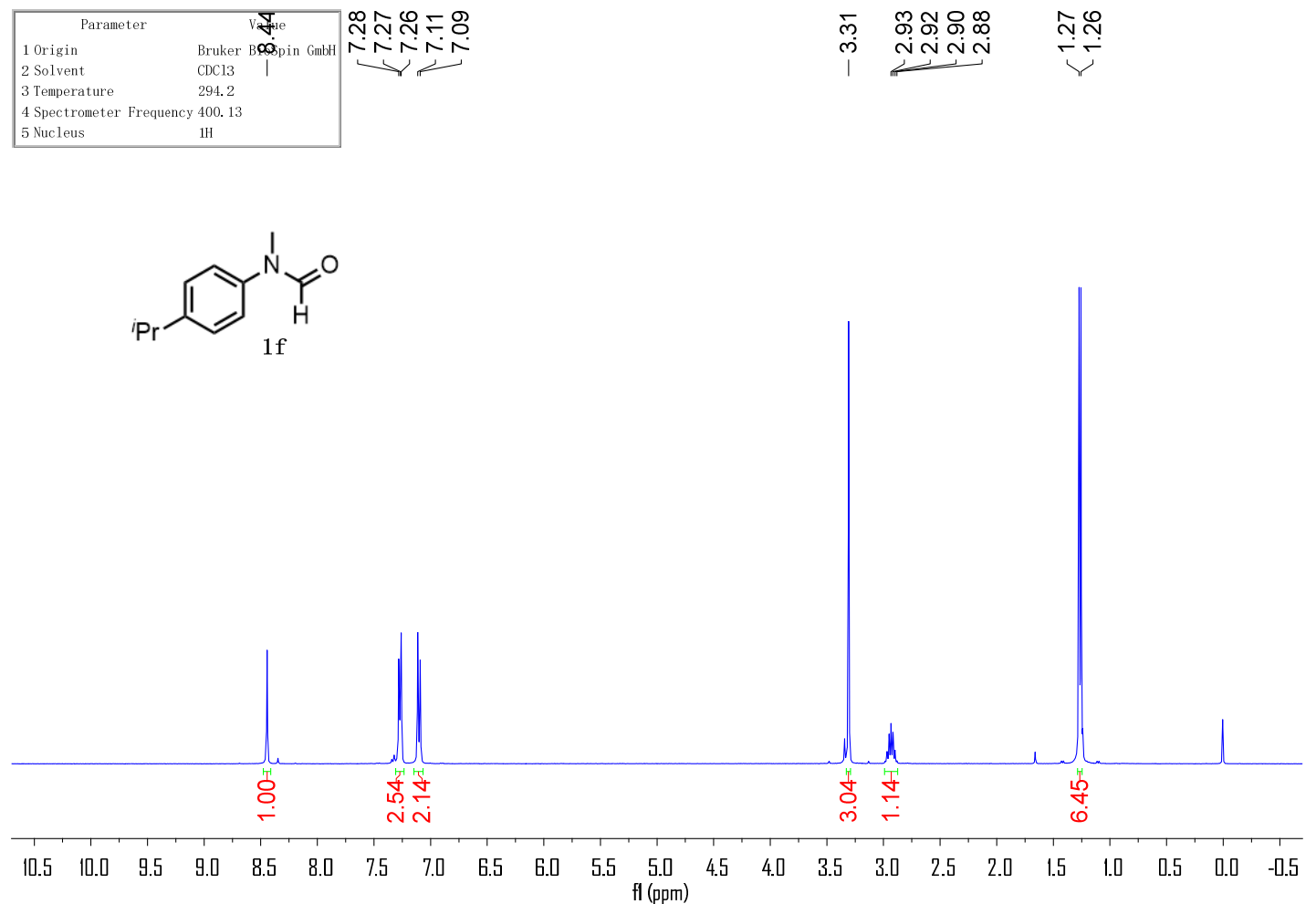

\begin{tabular}{|c|c|c|c|c|c|}
\hline Parameter & Value $\stackrel{Q}{+}$ & & 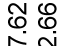 & 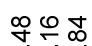 & ren \\
\hline $\begin{array}{l}1 \text { Origin } \\
2 \text { Solvent }\end{array}$ & $\begin{array}{l}\text { Bruker BioSpin NobH } \\
\text { CDC13 }\end{array}$ & 于 & $\stackrel{N}{ָ}$ & Nペ & ల్లై \\
\hline 3 Temperature & 296.0 & | & | 1 & 4 & $>1$ \\
\hline 4 Spectrometer Frequency & 100.61 & & & & \\
\hline & $13 \mathrm{C}$ & & & & \\
\hline
\end{tabular}<smiles>CCCc1ccc(N(C)C(=O)I)cc1</smiles>

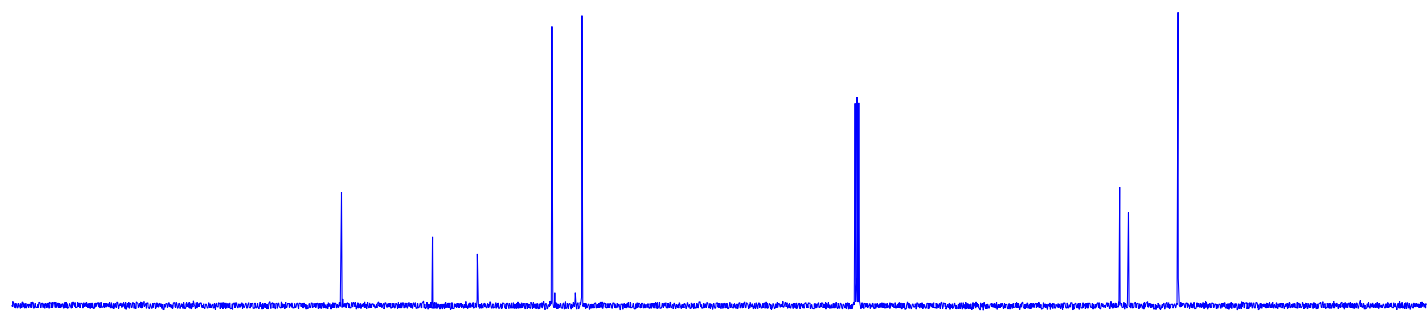

$\begin{array}{llllllllllllllllllllllll}210 & 200 & 190 & 180 & 170 & 160 & 150 & 140 & 130 & 120 & 110 & 100 & 50 & 80 & 70 & 60 & 50 & 40 & 30 & 20 & 10 & 0 & -10\end{array}$ 


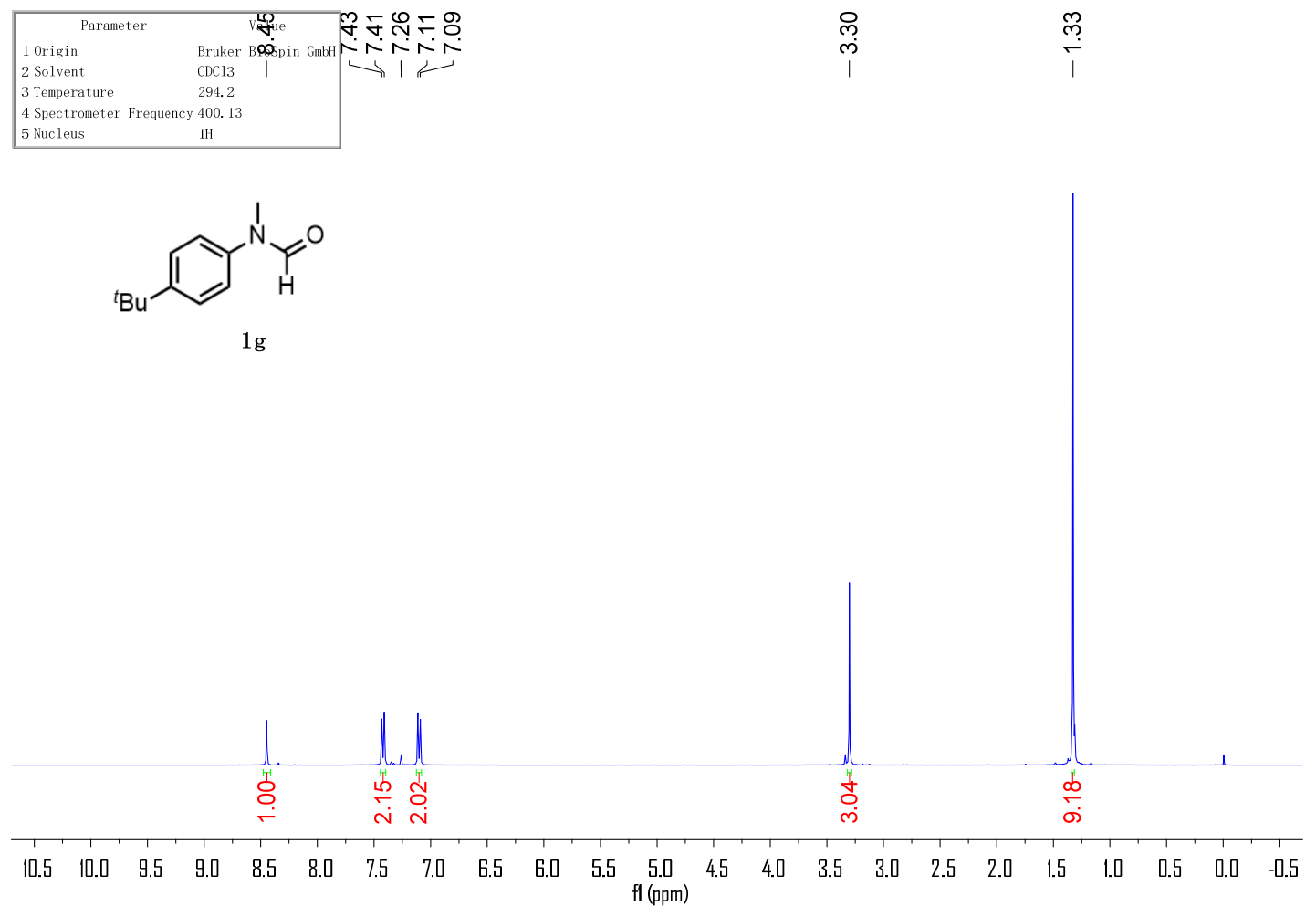

\begin{tabular}{|c|c|c|c|c|c|c|}
\hline Parameter & Value $\stackrel{0}{+}$ & م & $\stackrel{\infty}{N}$ & 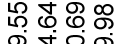 & $\stackrel{\infty}{\rightarrow} \stackrel{\infty}{\leftarrow}$ & $\ddot{\sim} \bar{v}$ \\
\hline $\begin{array}{l}1 \text { Origin } \\
2 \text { Solvent }\end{array}$ & $\begin{array}{l}\text { Bruker BioSpin @ibH } \\
\text { CDCl3 }\end{array}$ & ถి & $\stackrel{\text { Y }}{\mathfrak{r}}$ & 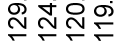 & NRQ & ले लू \\
\hline 3 Temperature & 296.0 & i & i & 11 & 4 & $<1$ \\
\hline $\begin{array}{l}4 \text { Spectrometer Frequency } \\
5 \text { Nucleus }\end{array}$ & $\begin{array}{l}100.61 \\
13 \mathrm{C}\end{array}$ & & & & & \\
\hline
\end{tabular}<smiles>CN(C=O)c1ccc(Br)cc1</smiles>

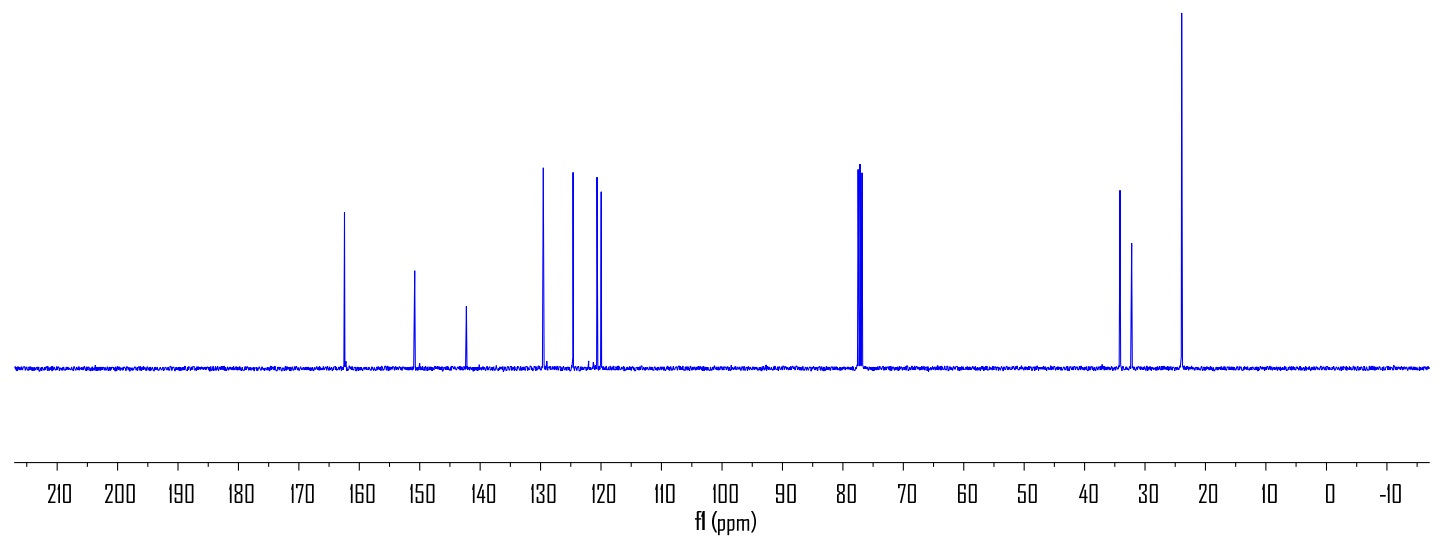




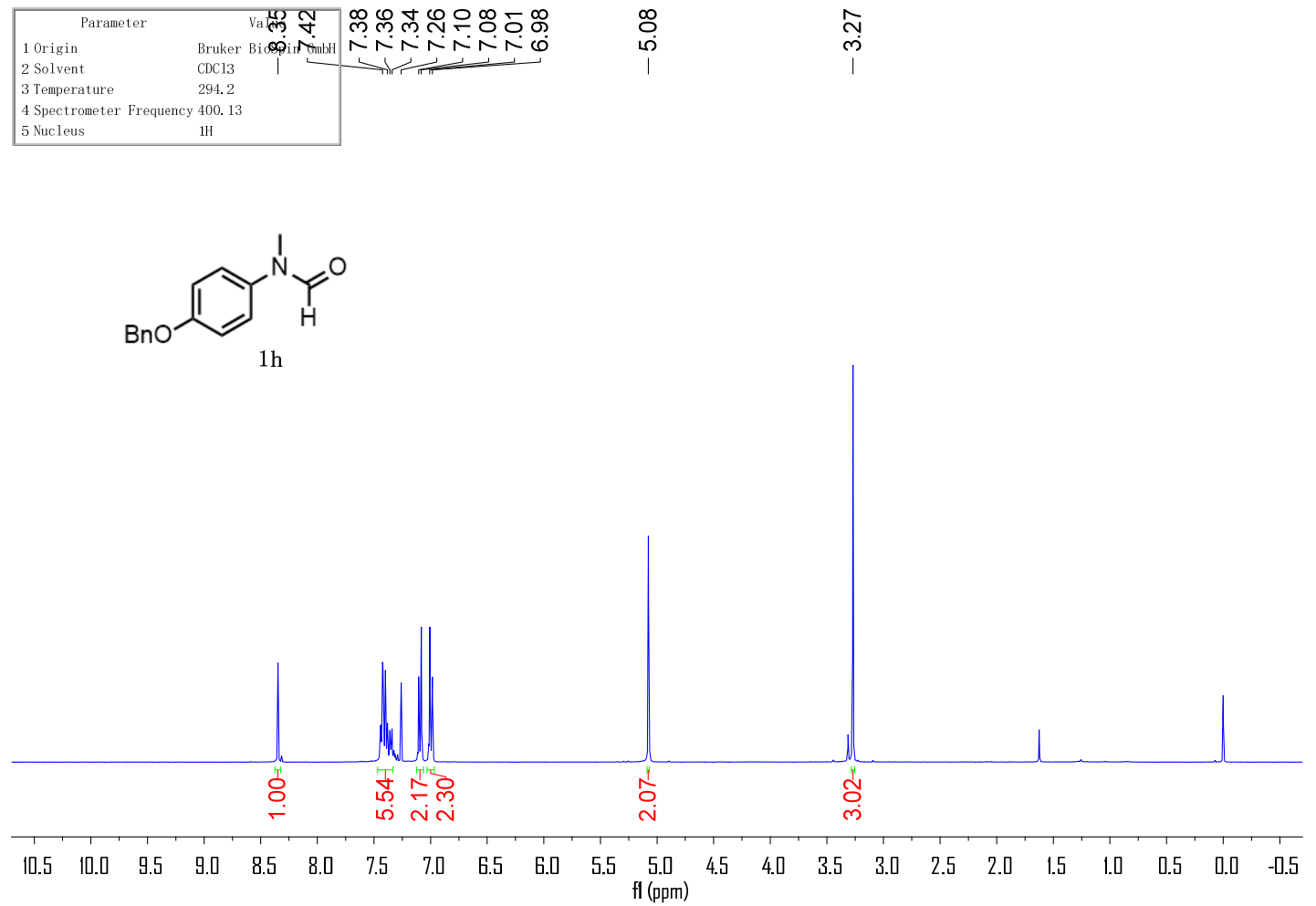<smiles>CN(C(=O)c1ccc(OCc2ccccc2)cc1)c1ccccc1</smiles>

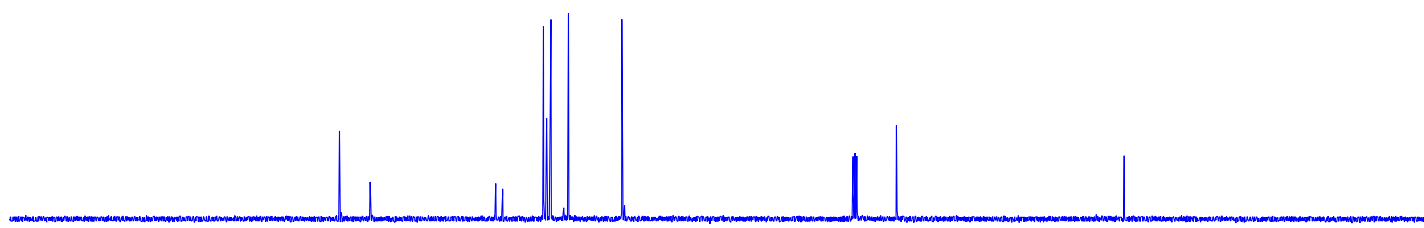

$\begin{array}{llllllllllllllllllllllllllll}210 & 200 & 190 & 180 & 170 & 160 & 150 & 140 & 130 & 120 & 110 & 100 & 50 & 80 & 70 & 60 & 50 & 40 & 30 & 20 & 10 & 0 & -10\end{array}$ 


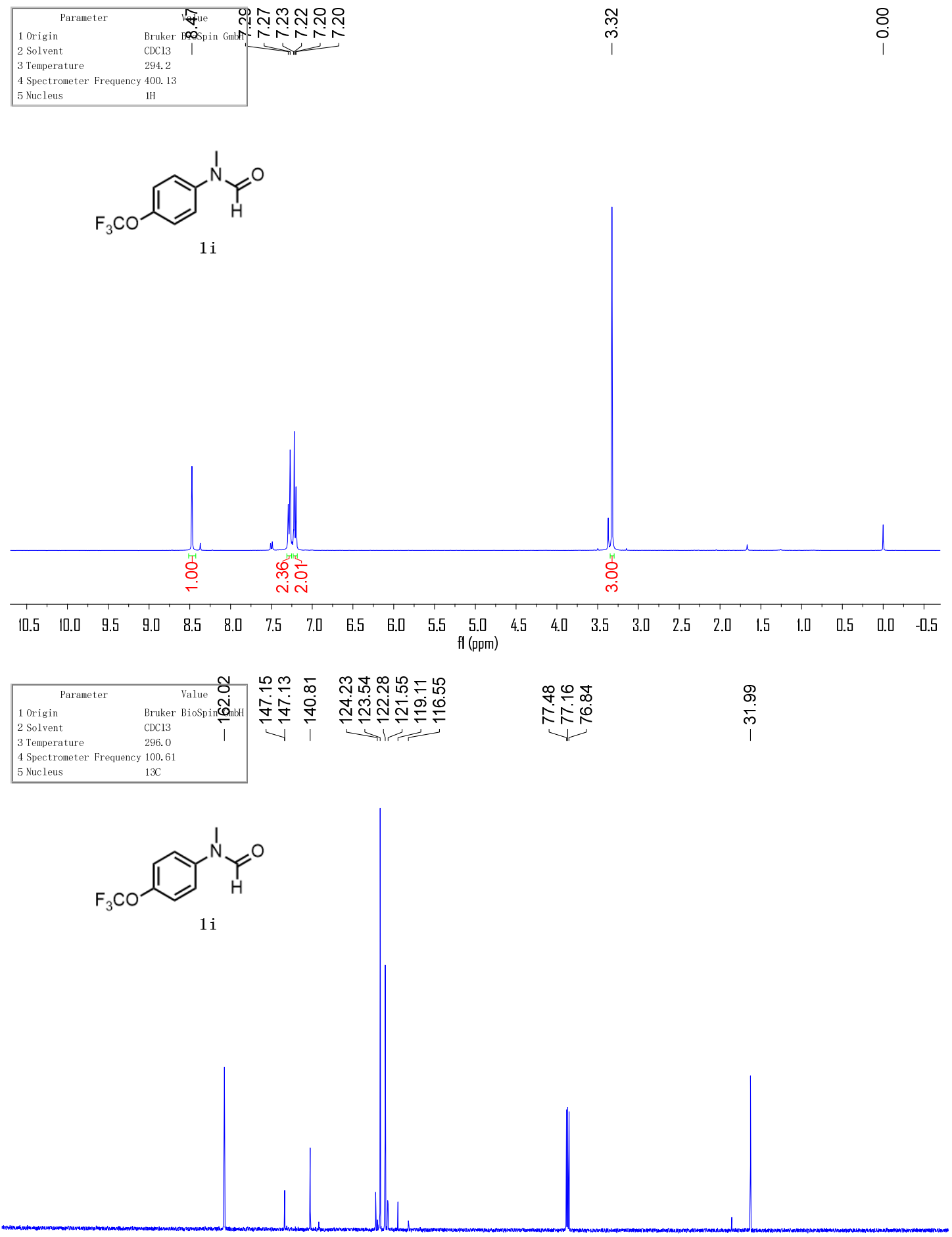

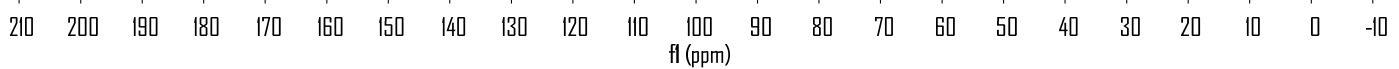




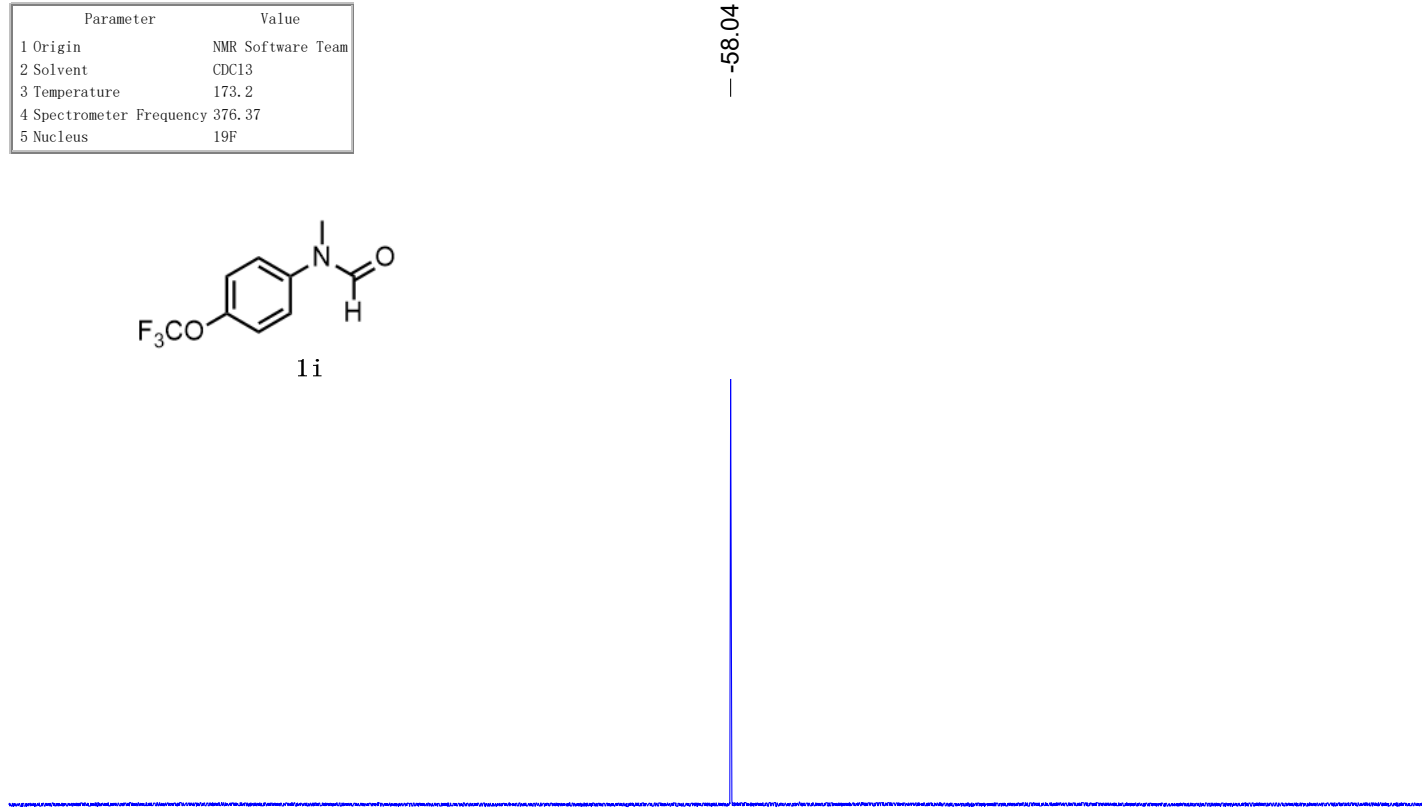

$\begin{array}{lllllllllllllllllllll}180 & 160 & 140 & 120 & 100 & 80 & 60 & 40 & 20 & 0 & -20 & -40 & -60 & -80 & -100 & -140 & -160 & -200 & -240 & -280\end{array}$

\begin{tabular}{|c|c|}
\hline Parameter & Valug \\
\hline 1 Origin & Bruker Bios9an GmbH \\
\hline 2 Solvent & $\mathrm{CDCl} 3$ \\
\hline 3 Temperature & 294.2 \\
\hline 4 Spectrometer Fre & 400.13 \\
\hline 5 Nucleus & $1 \mathrm{H}$ \\
\hline
\end{tabular}

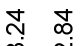

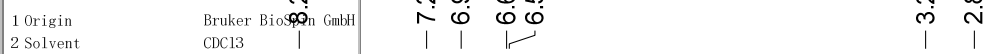

5 Nucleus<smiles>CNc1ccc(N(C)C=O)cc1</smiles>

$1 \mathrm{j}$

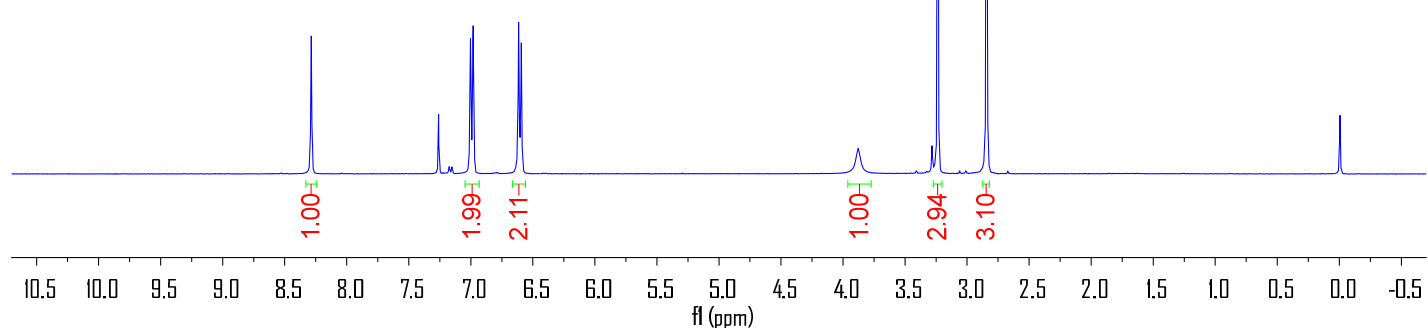


<smiles>CN(C)c1ccc(N(C)C=O)cc1</smiles>

$1 \mathrm{j}$

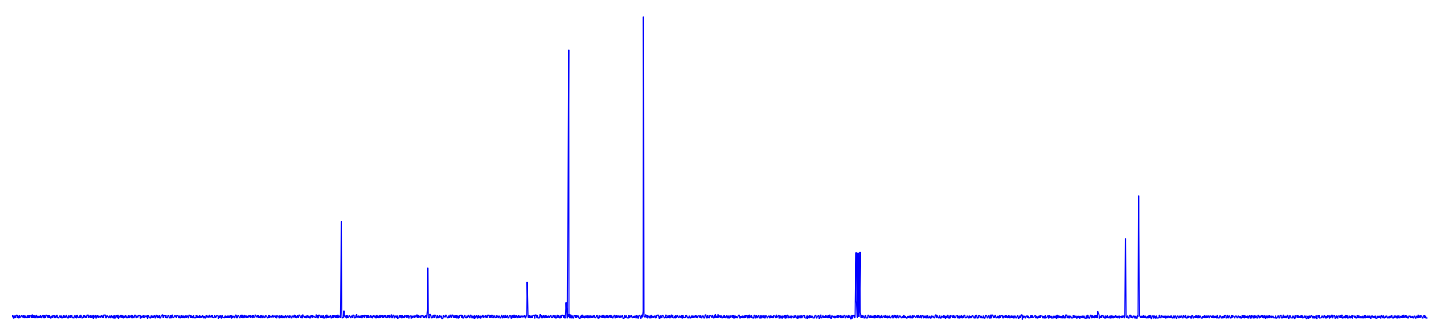

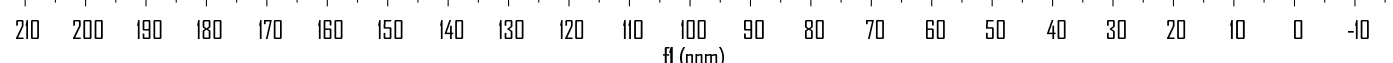

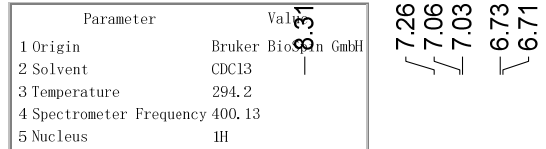

ขึำ

mi i<smiles>CN(C)c1ccc(N(C)C=O)cc1</smiles>

$1 \mathrm{k}$

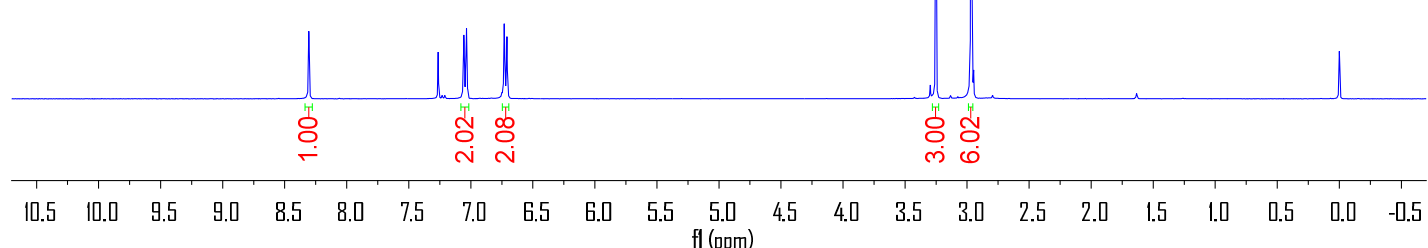




\begin{tabular}{|c|c|c|c|c|c|c|}
\hline Parameter & Value $\underset{0}{0}$ & กุ & 우 & ठా & $\infty \varphi$ & $\mp$ \\
\hline $\begin{array}{l}1 \text { Origin } \\
2 \text { Solvent }\end{array}$ & Bruker BioSpin ชึ่bH & ஒ் & $\dot{m} \stackrel{\sim}{\sim}$ & $\stackrel{\mathfrak{N}}{=}$ & 走电 & $\hat{\circ}$ \\
\hline $\begin{array}{l}2 \text { Solvent } \\
3 \text { Temperature }\end{array}$ & $\begin{array}{l}\mathrm{CDCl} 3 \\
296.0\end{array}$ & 5 & & 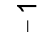 & Ni & + \\
\hline 4 Spectrometer Frequeno & y 100.61 & & & & & \\
\hline 5 Nucleus & $13 \mathrm{C}$ & & & & & \\
\hline
\end{tabular}

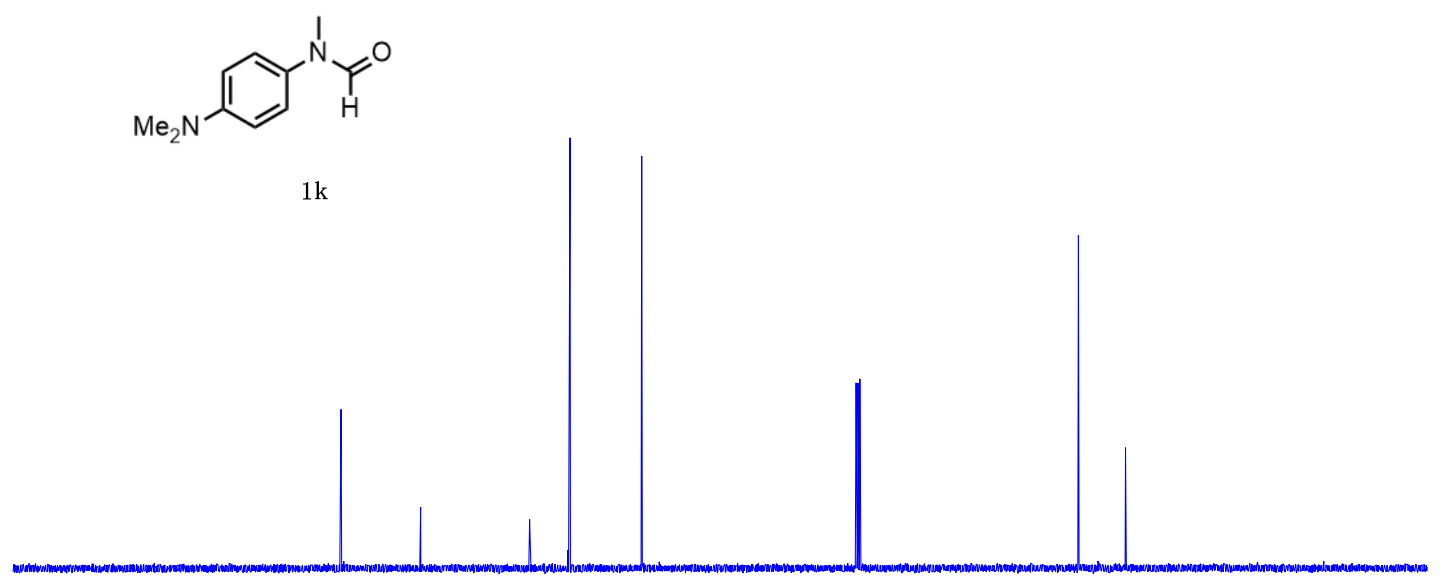

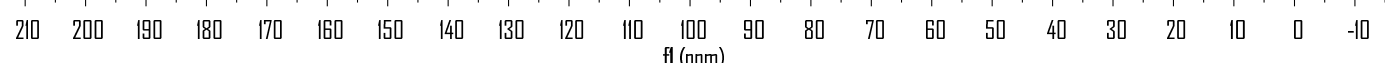

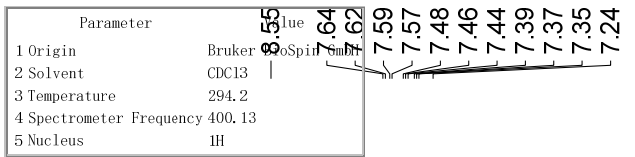<smiles>CN(C=O)c1ccc(-c2ccccc2)cc1</smiles>

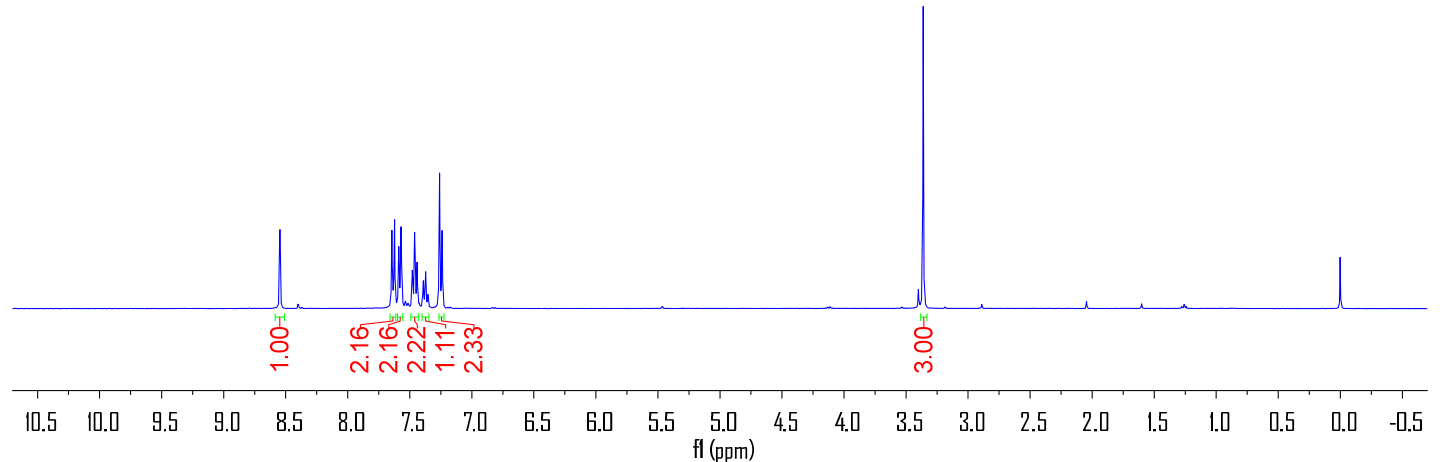



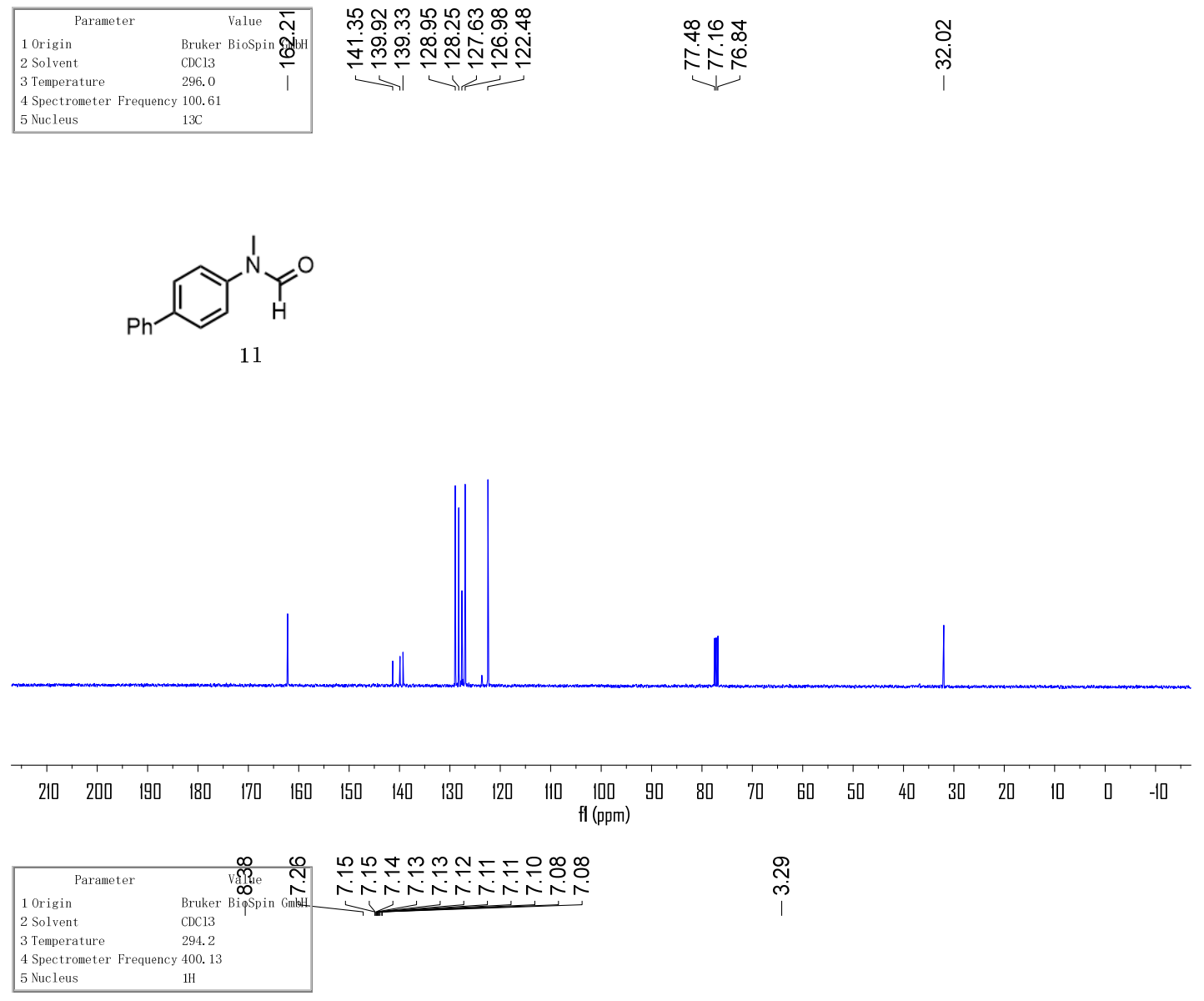<smiles>CN(C=O)c1ccc(F)cc1</smiles>

1m

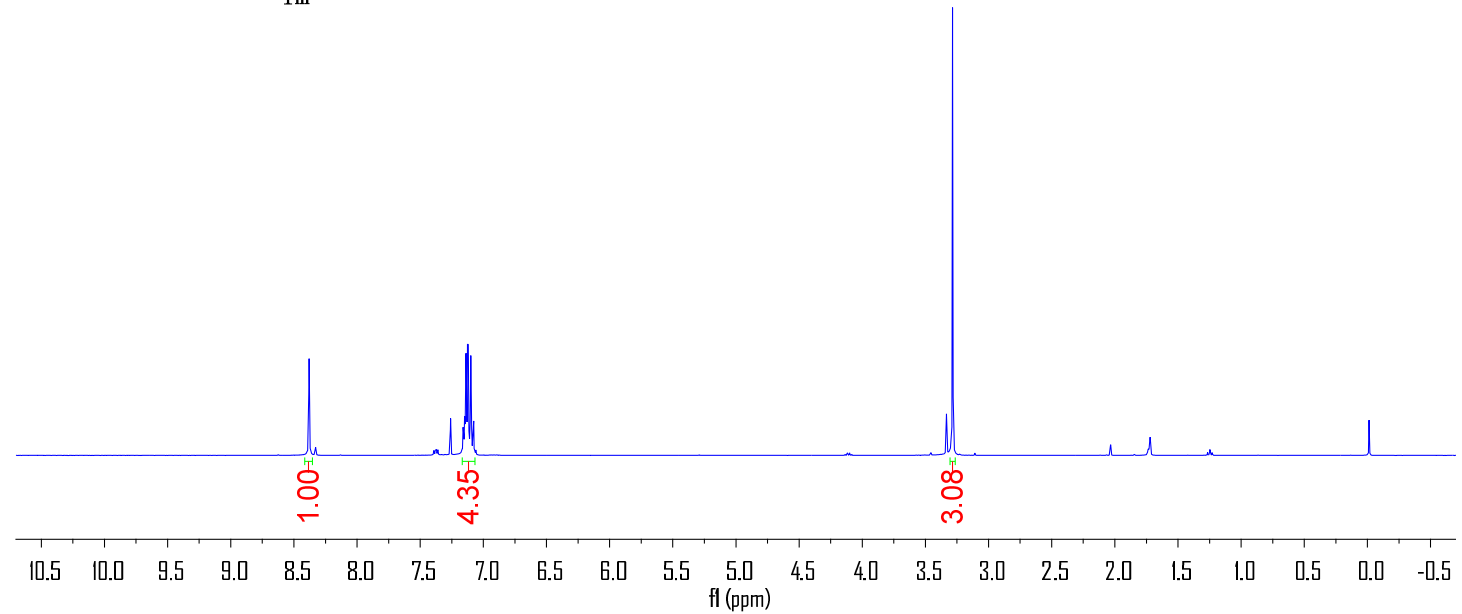



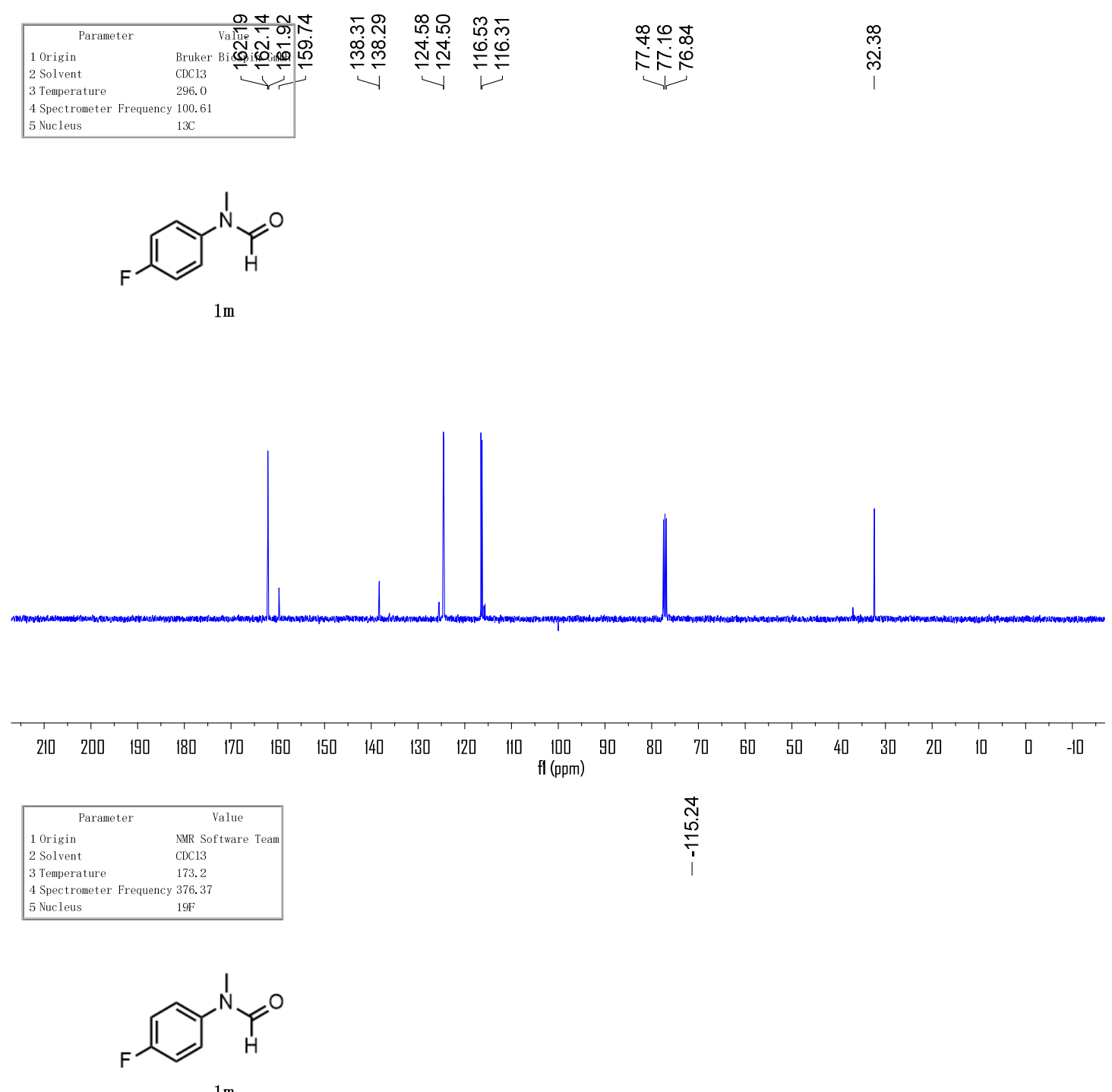

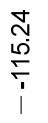

$1 \mathrm{~m}$

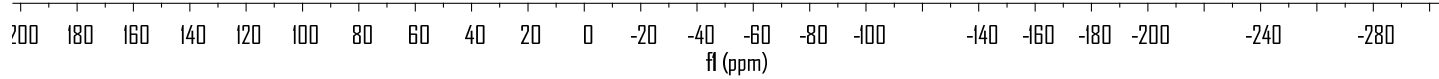




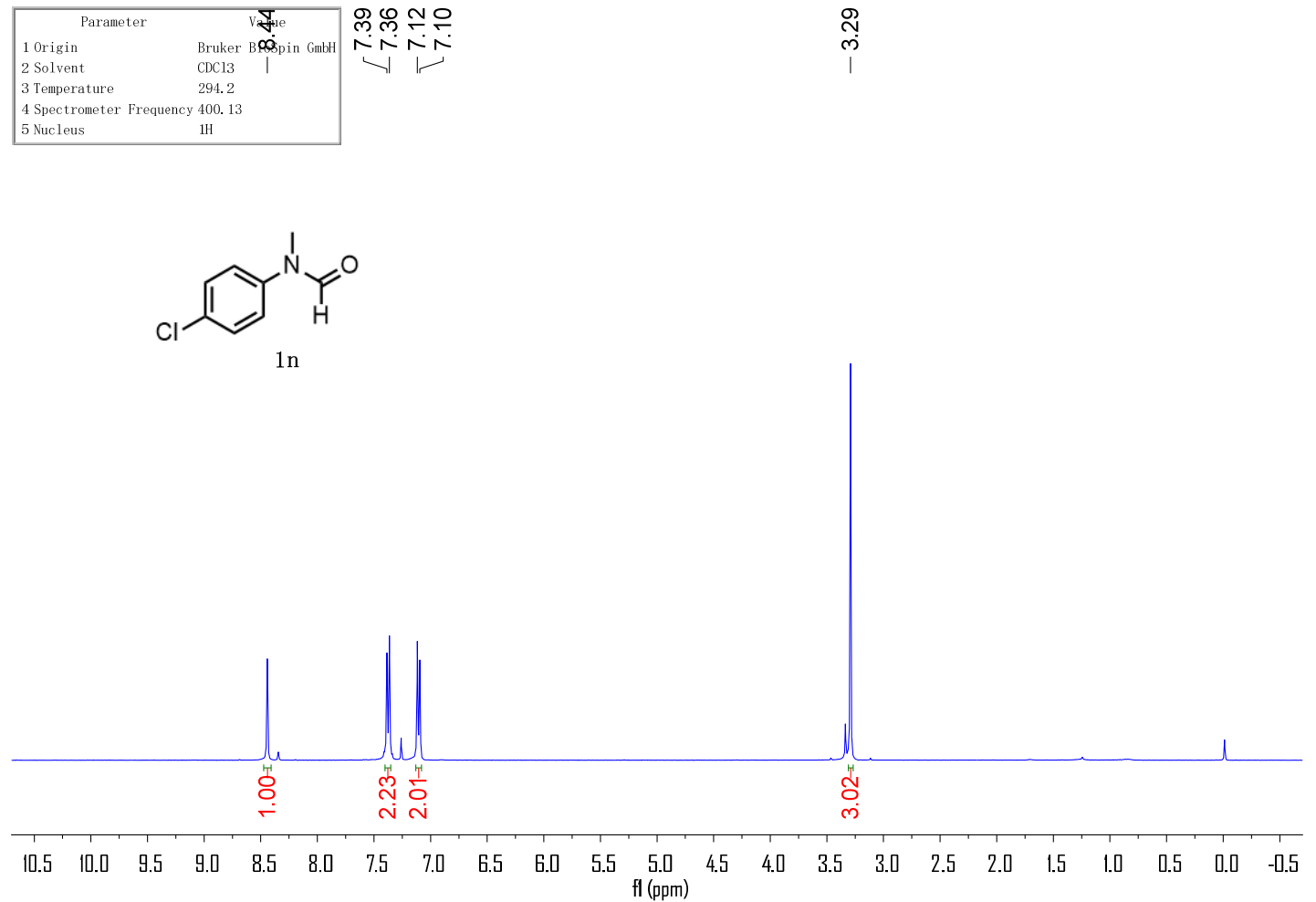

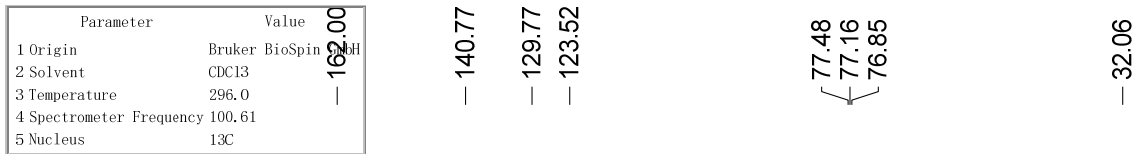<smiles>CN(C=O)c1ccc(Cl)cc1</smiles>

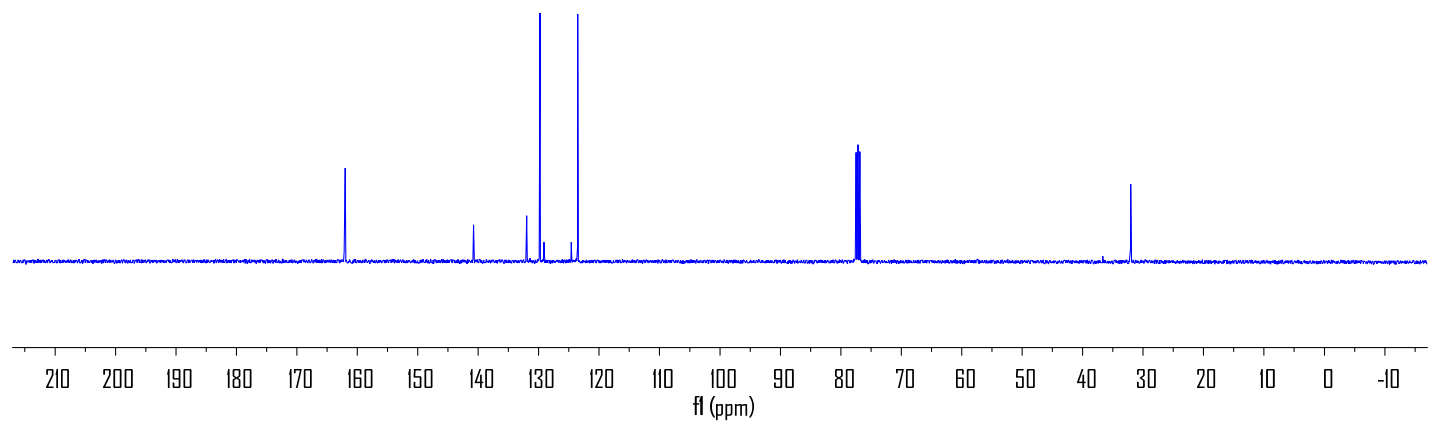


<smiles>CN(C=O)c1ccc(C(F)(F)F)cc1</smiles>

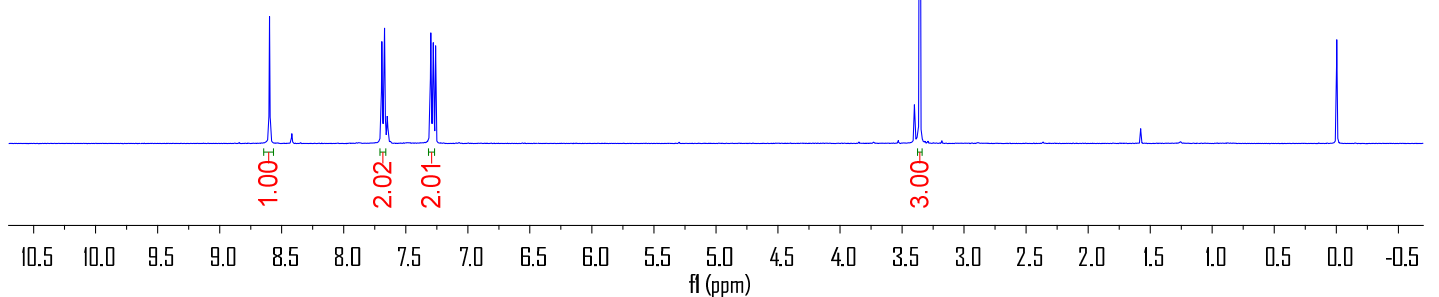

\section{స

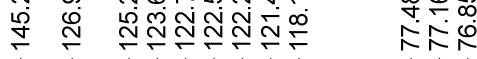

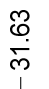

$\begin{array}{ll}2 \text { Solvent } & \text { CDCl3 } \\ 3 \text { Temperature } & 296.0\end{array}$

4 Spectrometer Frequency 100.

5 Nucleu.<smiles>CN(C=O)c1ccc(C(F)(F)F)cc1</smiles>

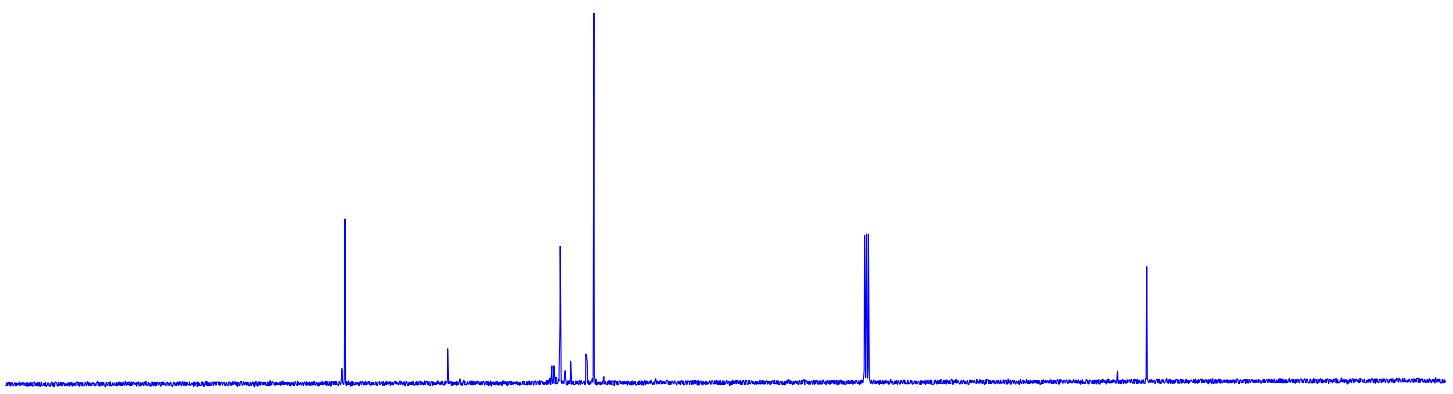

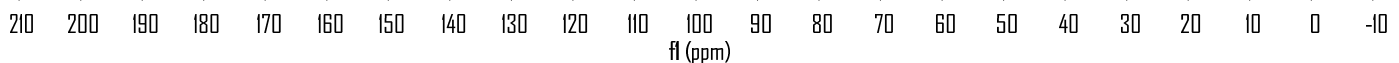




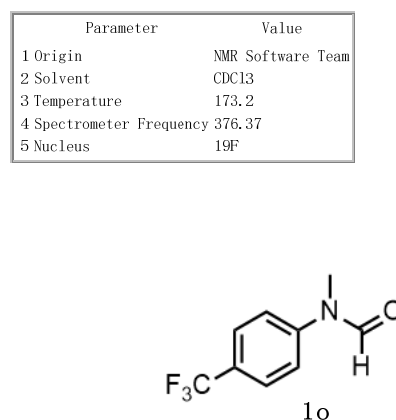

10

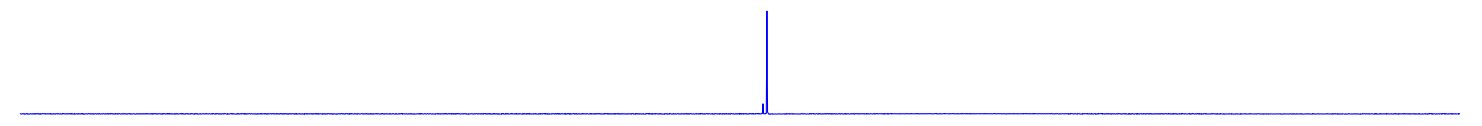

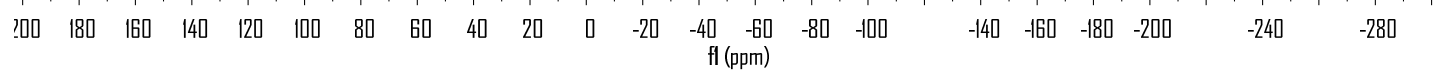

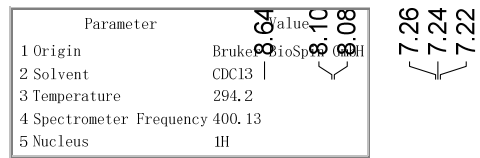<smiles></smiles>

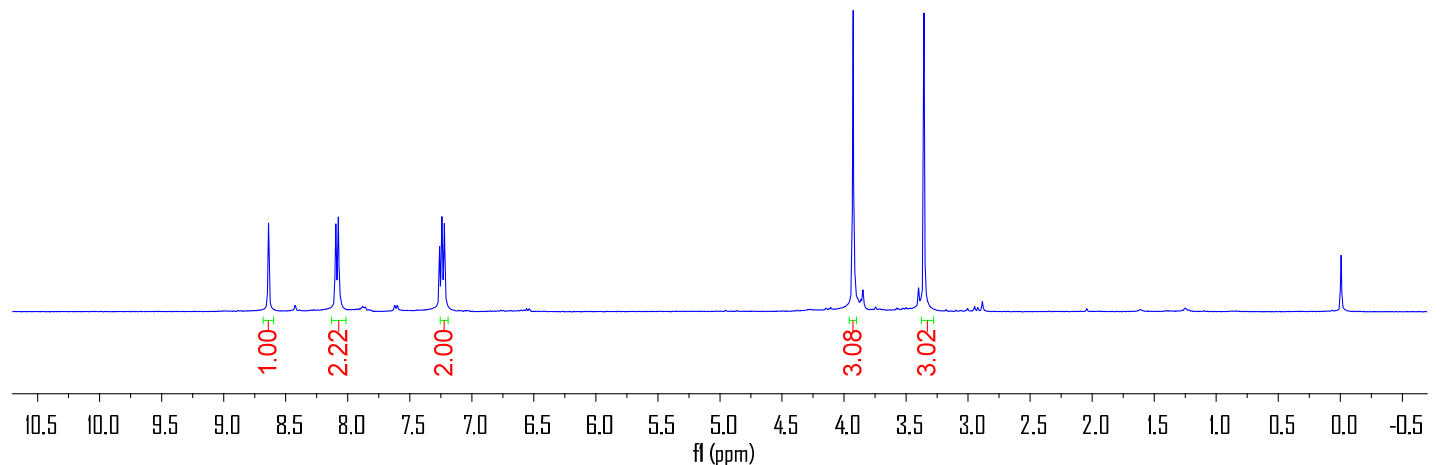




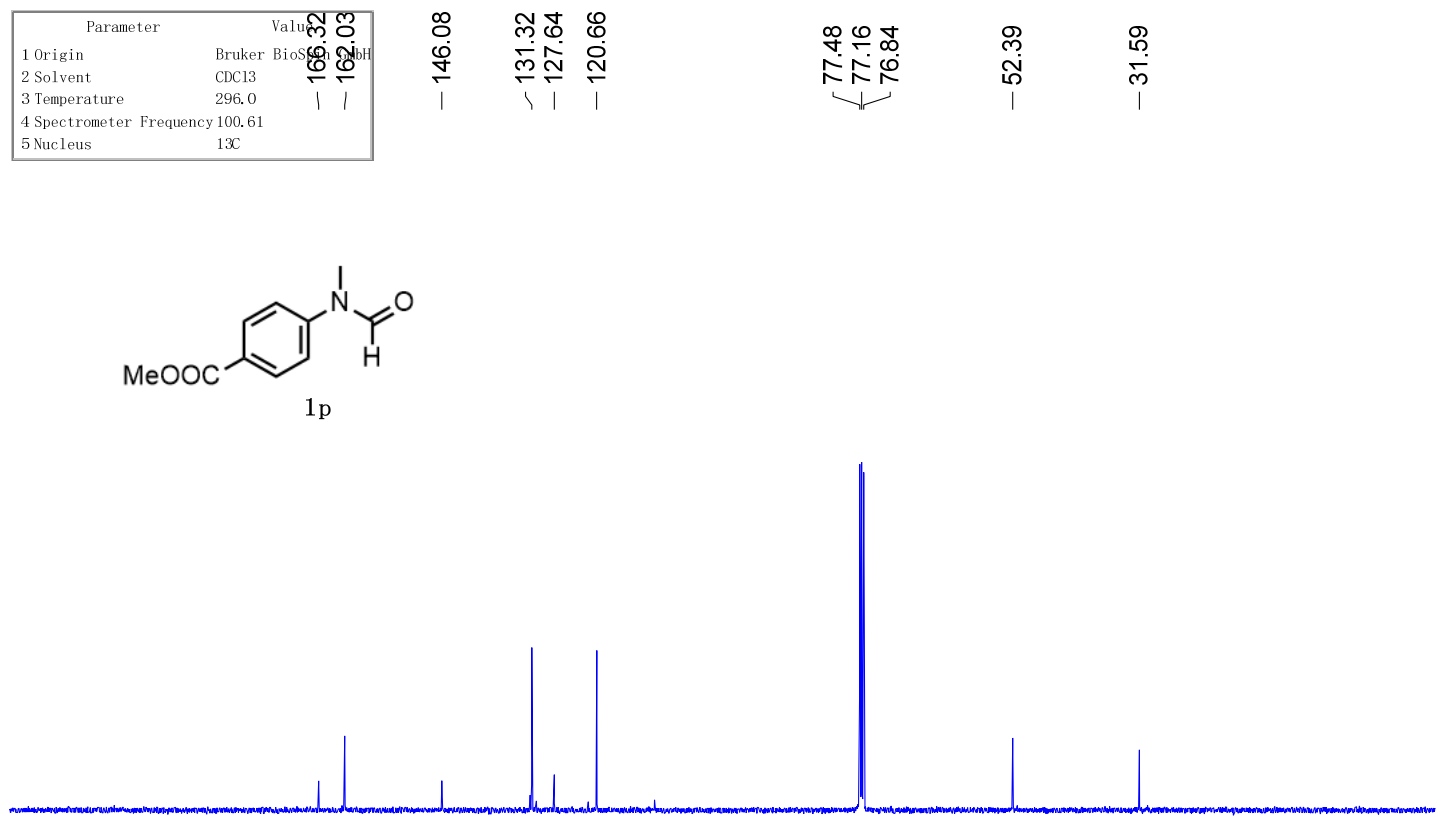

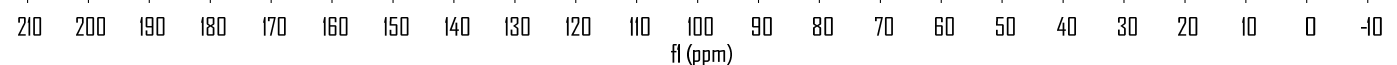

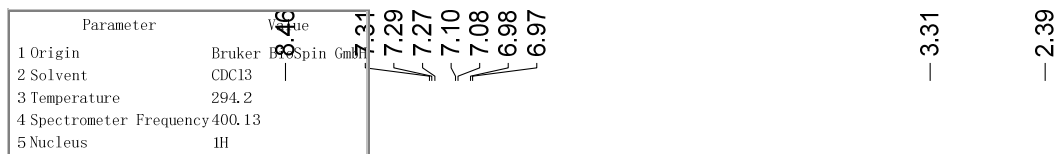<smiles>Cc1cccc(N(C)C=O)c1</smiles>

$1 \mathrm{q}$

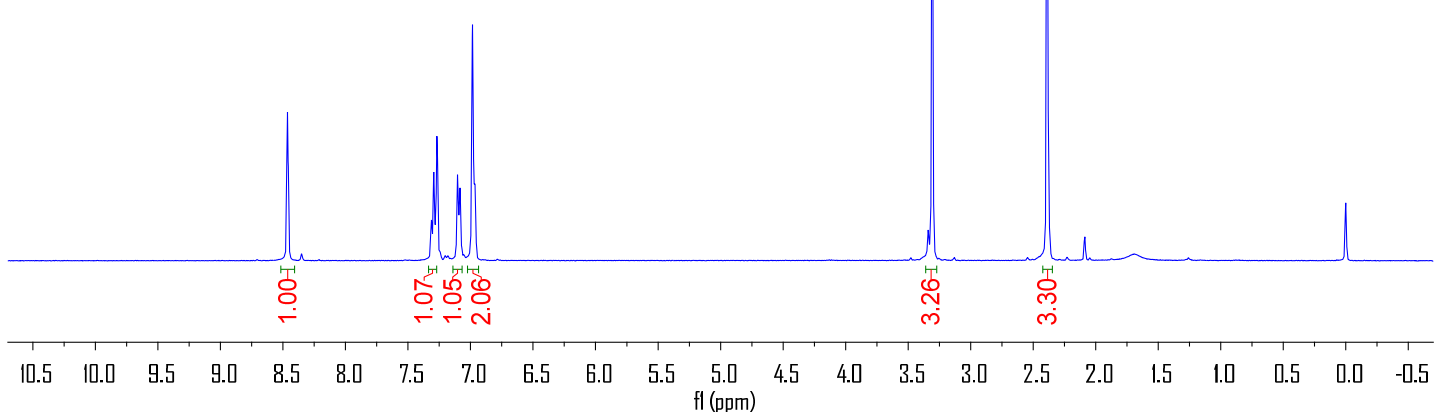



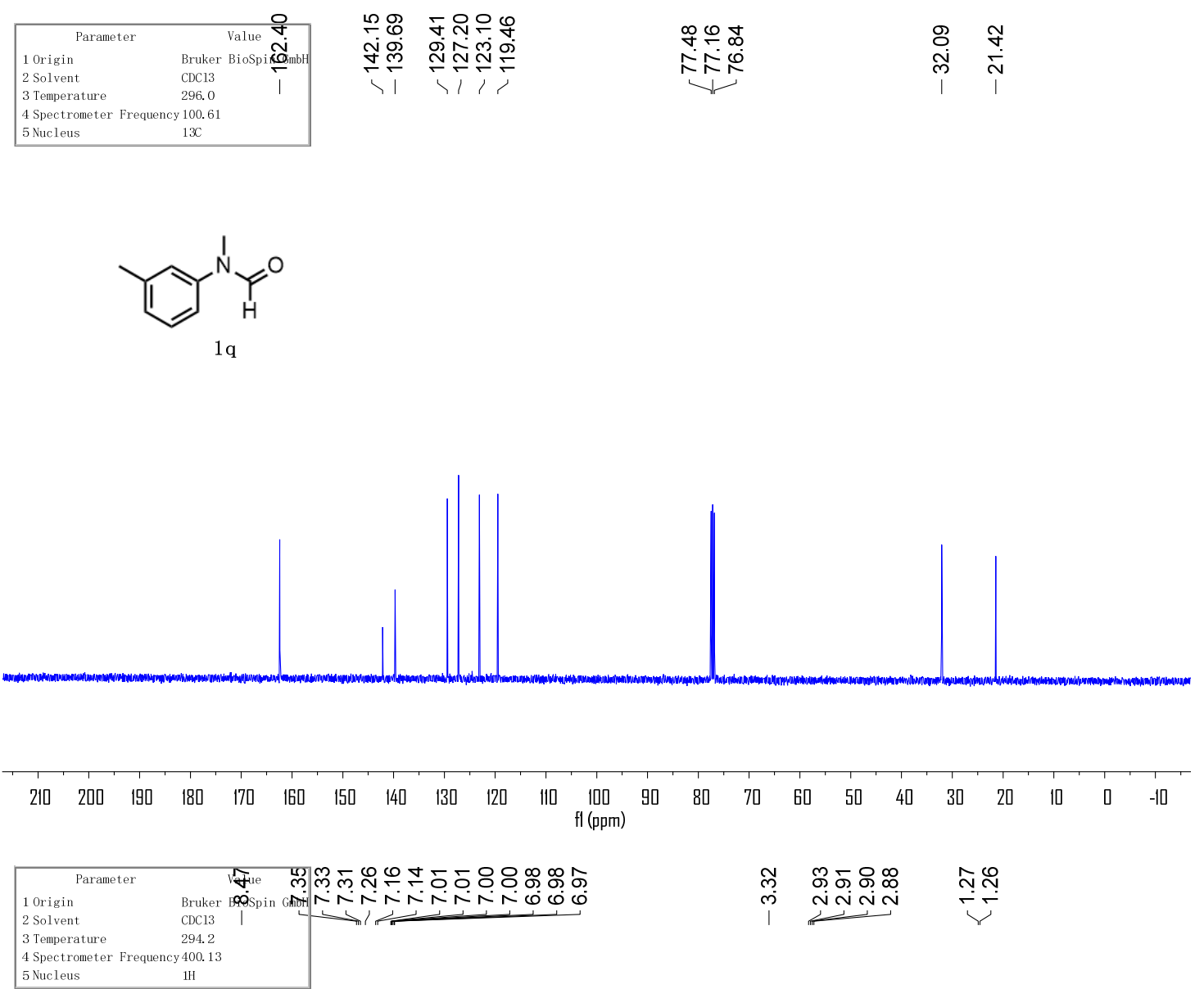

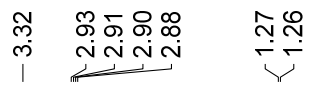

$1 \mathrm{H}$
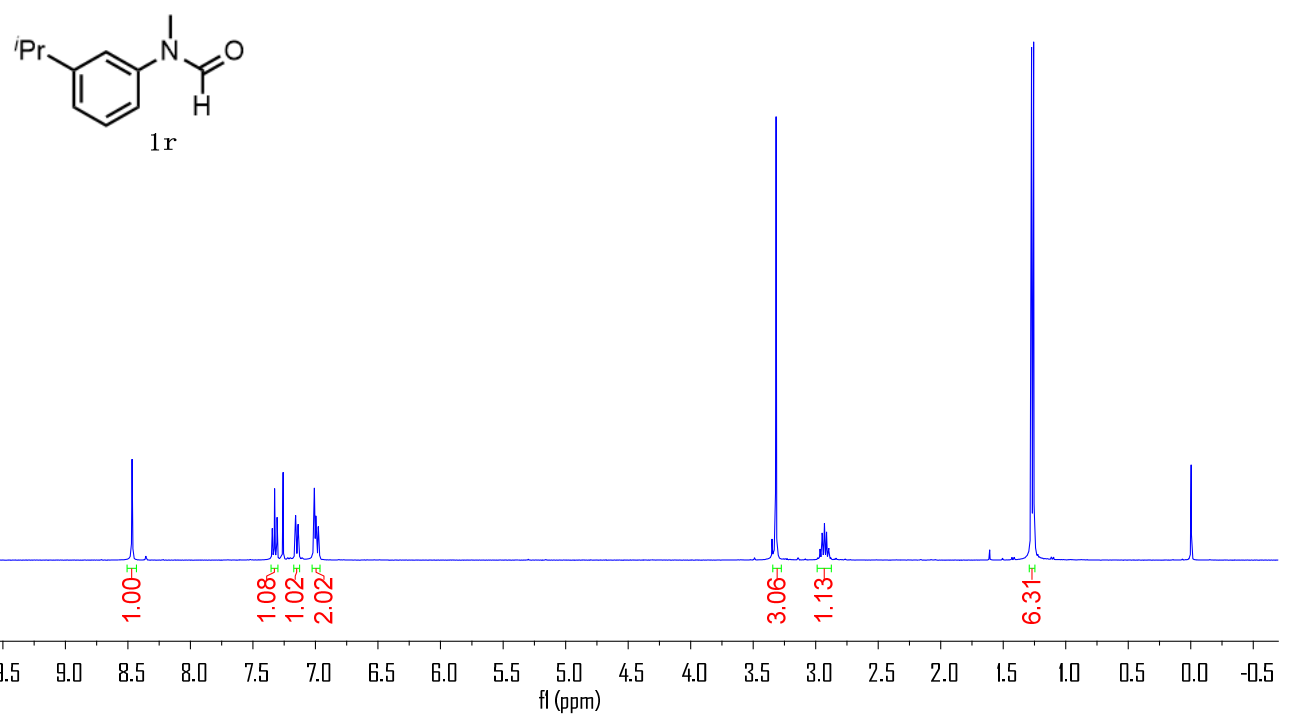


\begin{tabular}{|c|c|c|c|c|c|}
\hline Parameter & Value $\stackrel{\infty}{m}$ & م & 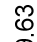 & గ్ర & เก \\
\hline $\begin{array}{l}1 \text { Origin } \\
2 \text { Solvent }\end{array}$ & $\begin{array}{l}\text { Bruker BioSpin बृं } \\
\text { CDCl3 }\end{array}$ & 守 & হై & $\stackrel{\oplus}{\stackrel{2}{2}}$ & ले \\
\hline $\begin{array}{l}3 \text { Temperature } \\
4 \text { Spectrometer Freq }\end{array}$ & $\begin{array}{c}296.0 \\
\text { y } 100.61\end{array}$ & i & I & 11 & 4 \\
\hline
\end{tabular}<smiles>CCCc1cccc(N(C)C(=O)[In])c1</smiles>

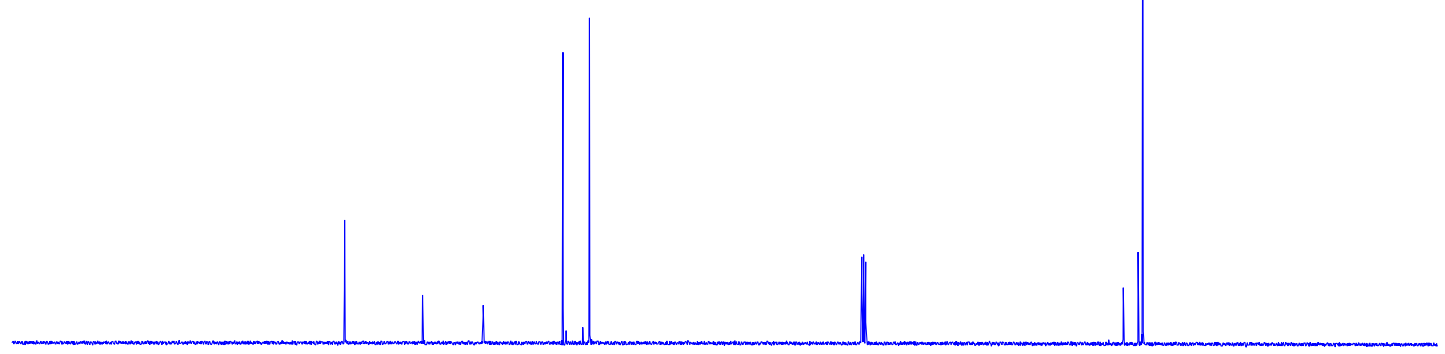

$\begin{array}{llllllllllllllllllllllllll}210 & 200 & 190 & 180 & 170 & 160 & 150 & 140 & 130 & 120 & 110 & 100 & 90 & 80 & 70 & 60 & 50 & 40 & 30 & 20 & 10 & 0 & -10\end{array}$

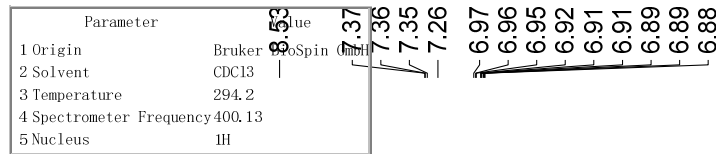

$\stackrel{\bar{m}}{\text { pं }}$<smiles>CN(C=O)c1cccc(F)c1</smiles>

$1 \mathrm{~s}$

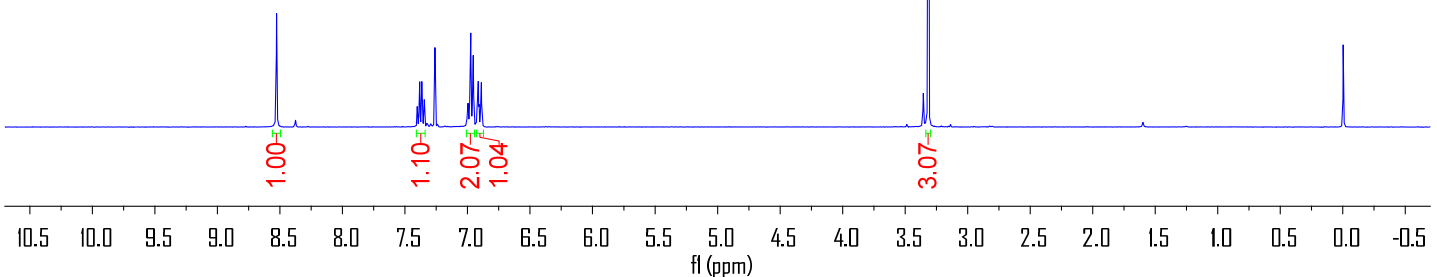




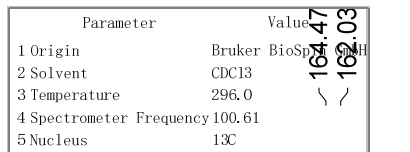<smiles>CN(C=O)c1cccc(F)c1</smiles>

$1 \mathrm{~s}$

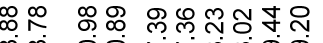

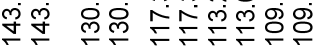

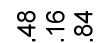

К下足

N

$\dot{m}$

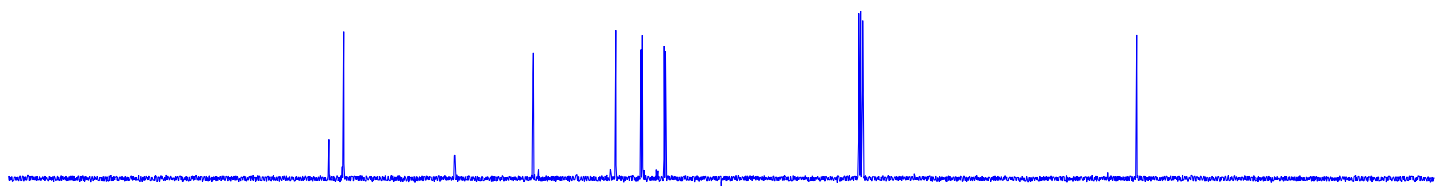

$\begin{array}{lllllllllllllllllllllll}210 & 200 & 190 & 180 & 170 & 160 & 150 & 140 & 130 & 120 & 110 & \begin{array}{c}100 \\ f 1(\mathrm{pm})\end{array} & 90 & 80 & 70 & 60 & 50 & 40 & 30 & 20 & 10 & 0 & -10\end{array}$

\begin{tabular}{|lc|}
\hline \multicolumn{1}{|c}{ Parameter } & \multicolumn{1}{c|}{ Value } \\
1 Origin & NMR Software Tean \\
2 Solvent & CDC13 \\
3 Temperature & 173.2 \\
4 Spectrometer & Frequency 376. 37 \\
5 Nucleus & $19 \mathrm{~F}$ \\
\hline
\end{tabular}

$\frac{9}{\stackrel{9}{0}}$<smiles>CN(C=O)c1cccc(F)c1</smiles>

$1 \mathrm{~s}$

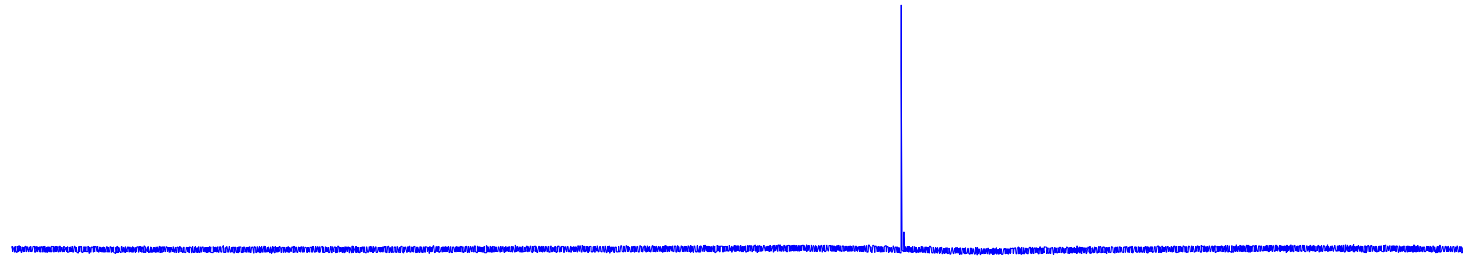

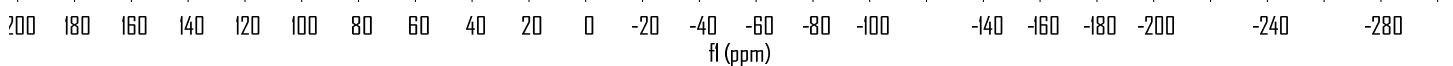




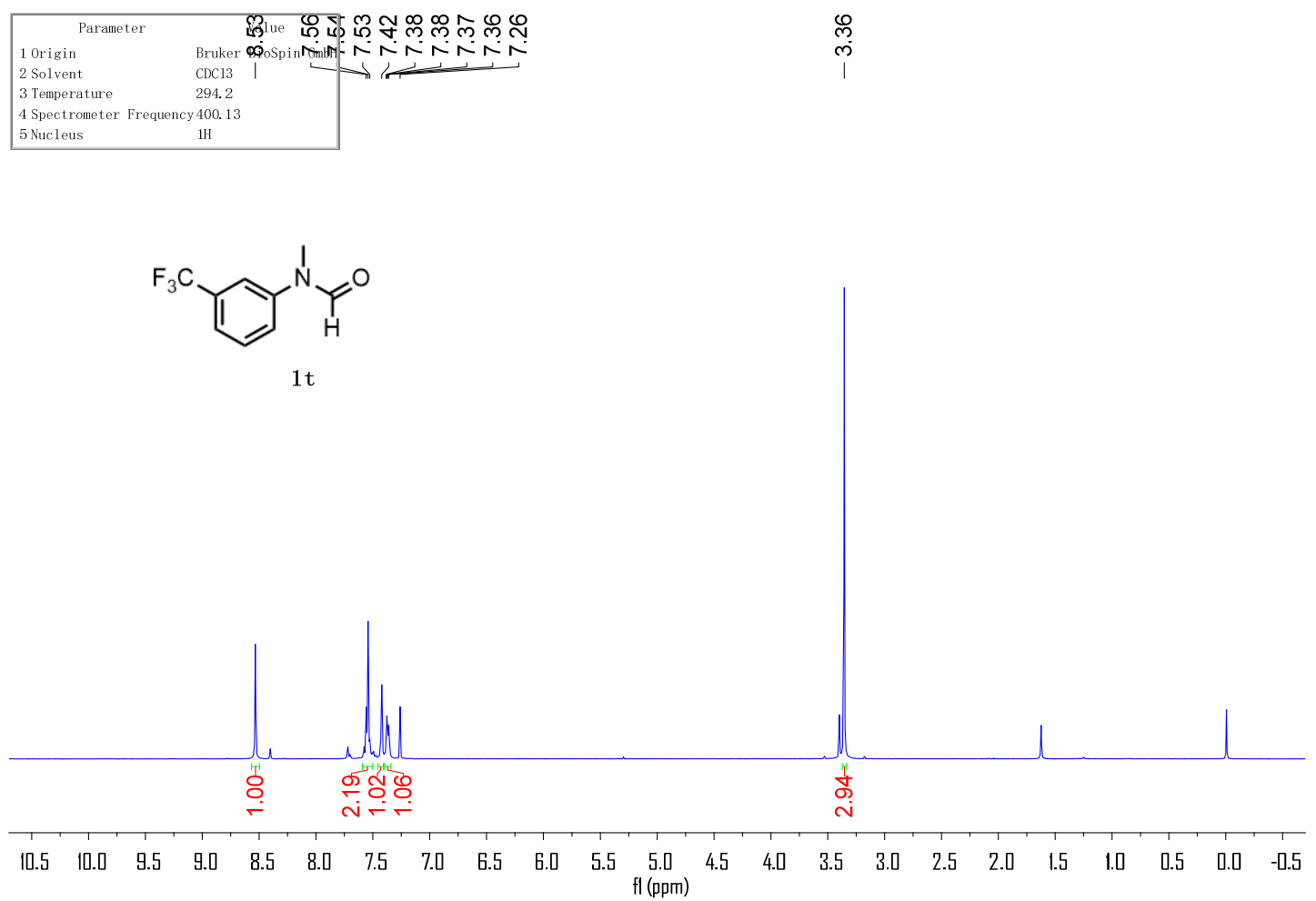<smiles>CN(C=O)c1cccc(C(F)(F)F)c1</smiles>

$1 \mathrm{t}$

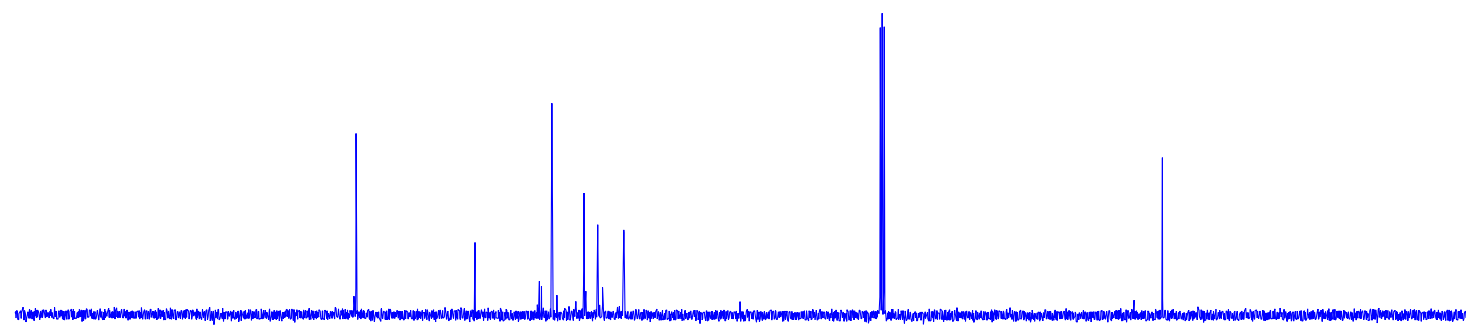

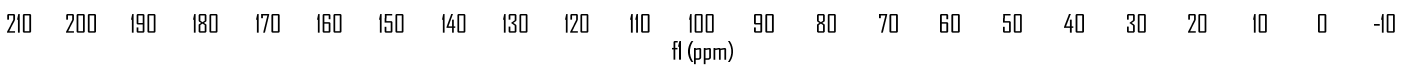


<smiles>CN(C(=O)c1cccc(C(F)(F)F)c1)c1ccccc1</smiles>

$1 \mathrm{t}$

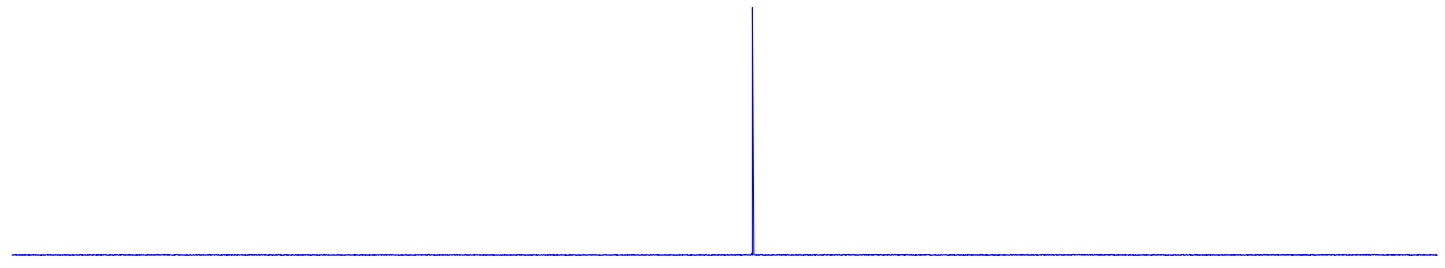

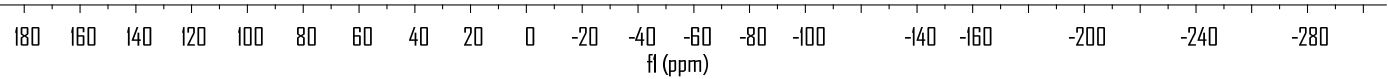

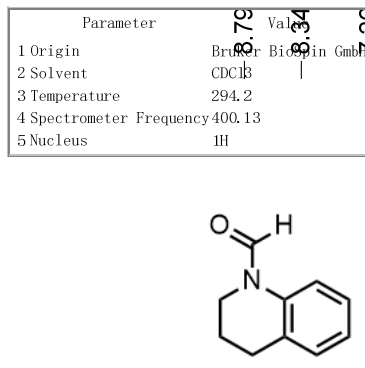

$1 \mathrm{u}$

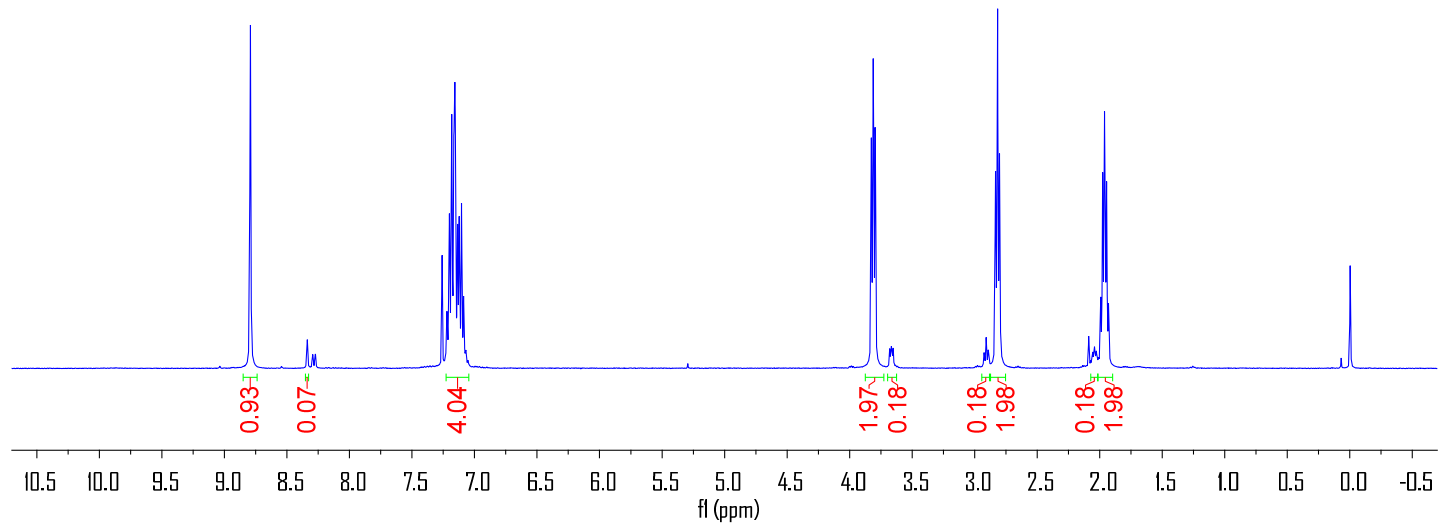




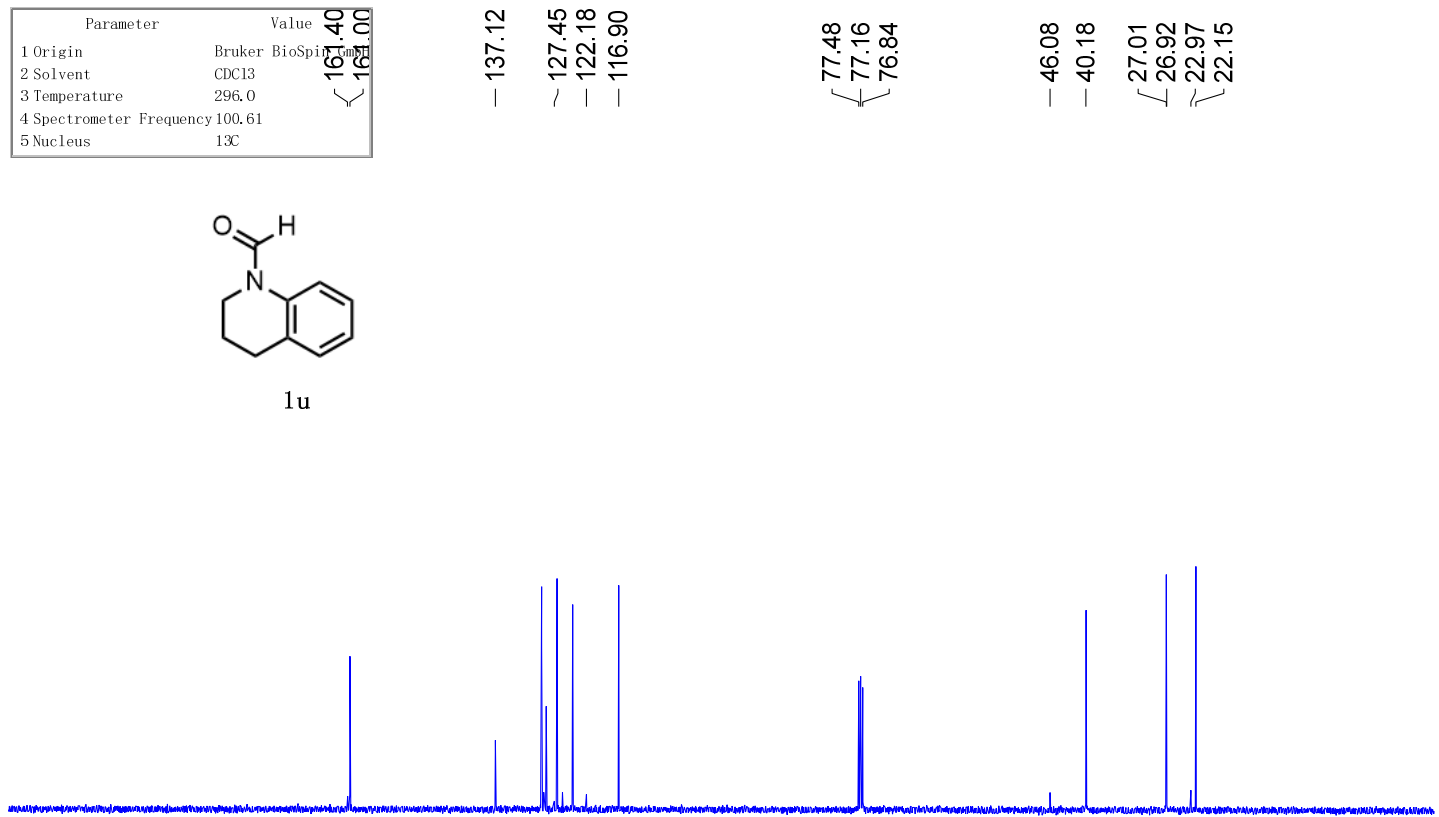

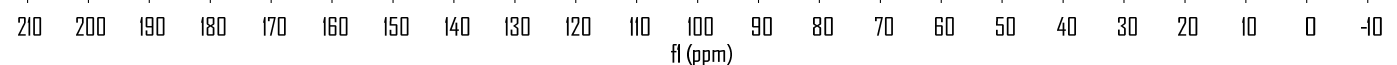

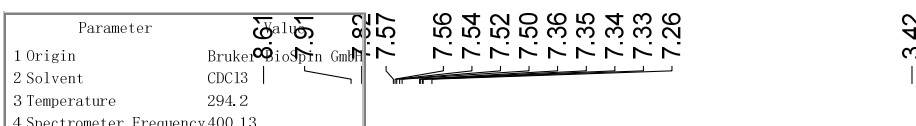

4 Spectrometer Frequency 400.13
5 Nucleus

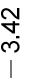<smiles>CN(C=O)c1ccc2ccccc2c1</smiles>

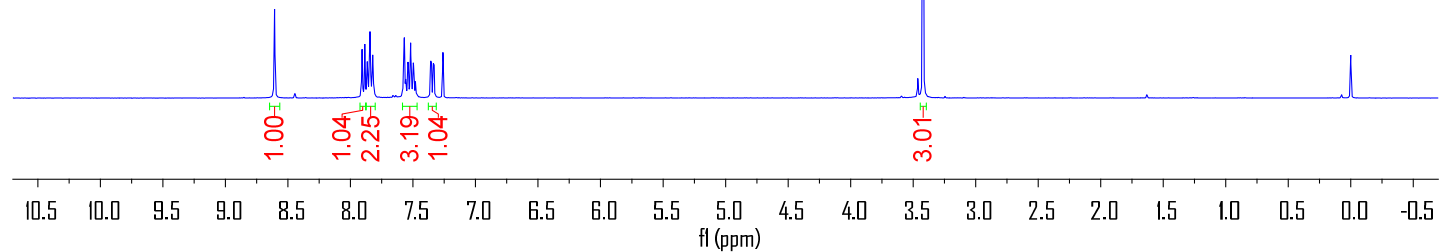




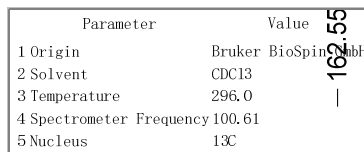

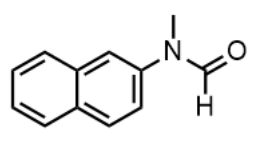

$1 \mathrm{v}$
운은

송

$\stackrel{\sim}{\sim}$

ले

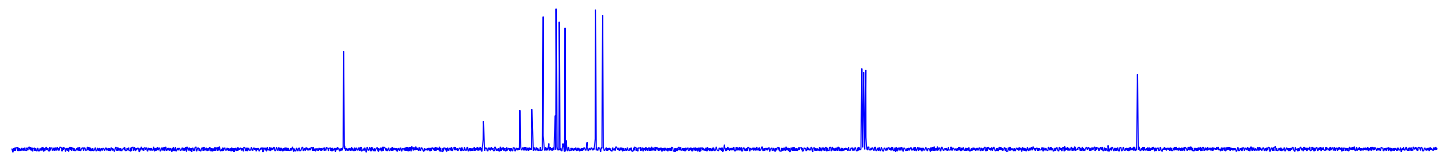

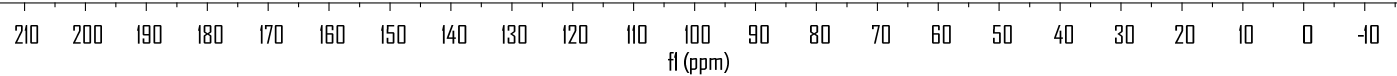

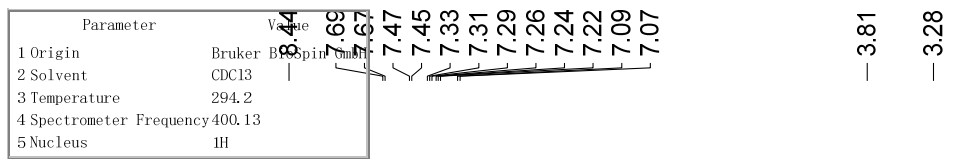<smiles>CN(C=O)NC1C=CC2=C3C=CC=C3CC2C1</smiles>

$1 \mathrm{w}$

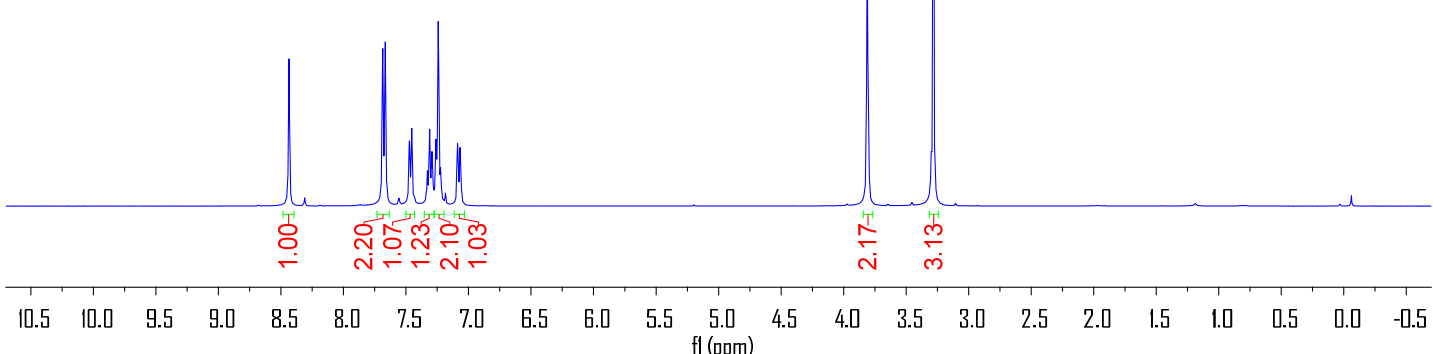




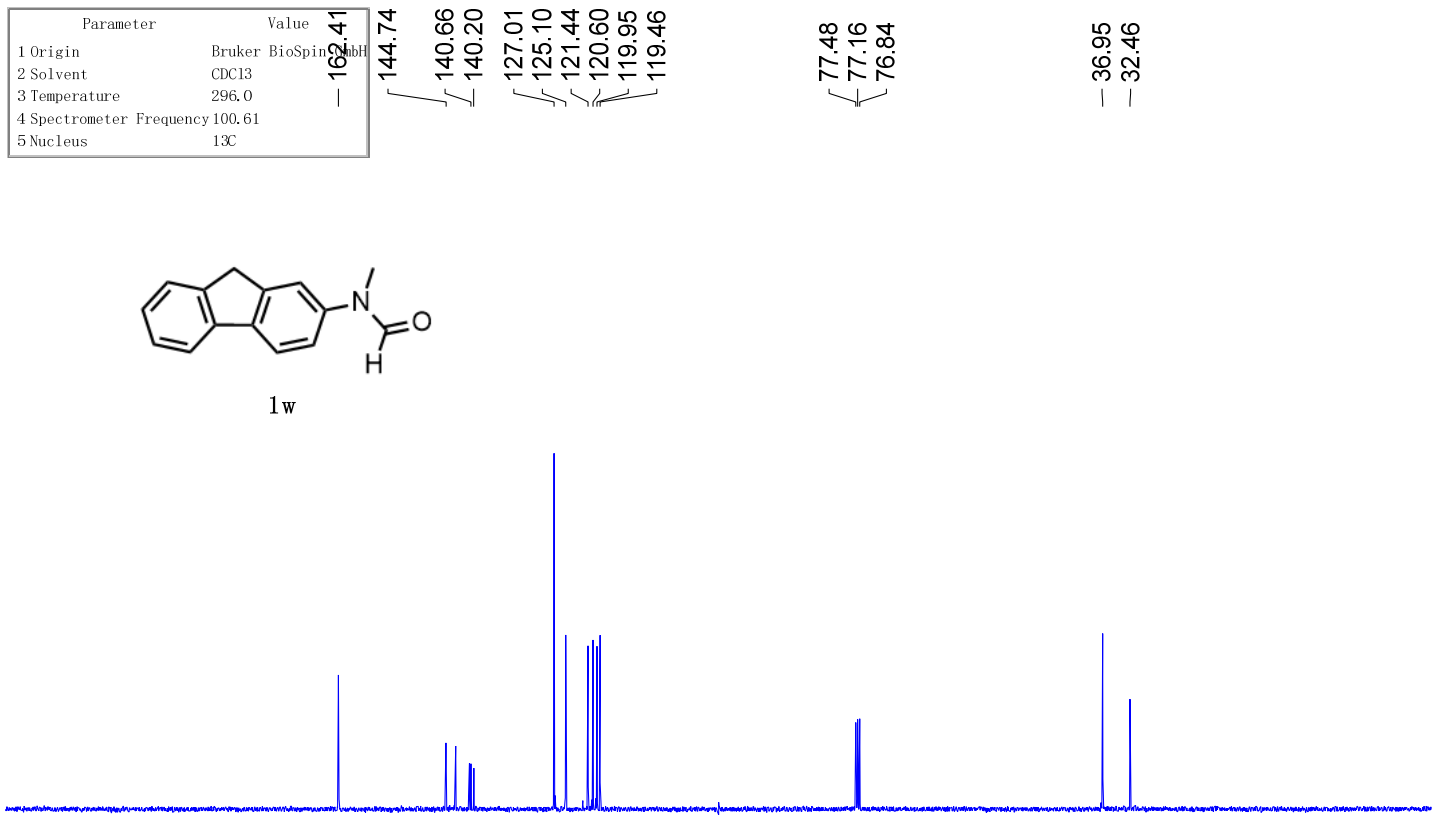

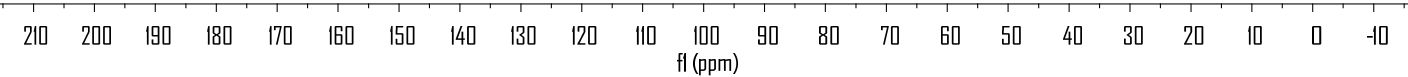

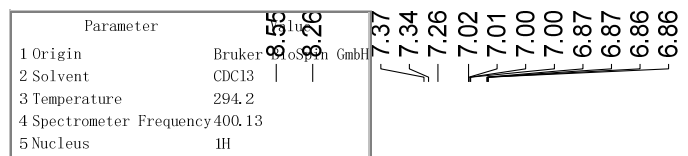

m

ले

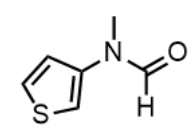

$1 \mathrm{x}$

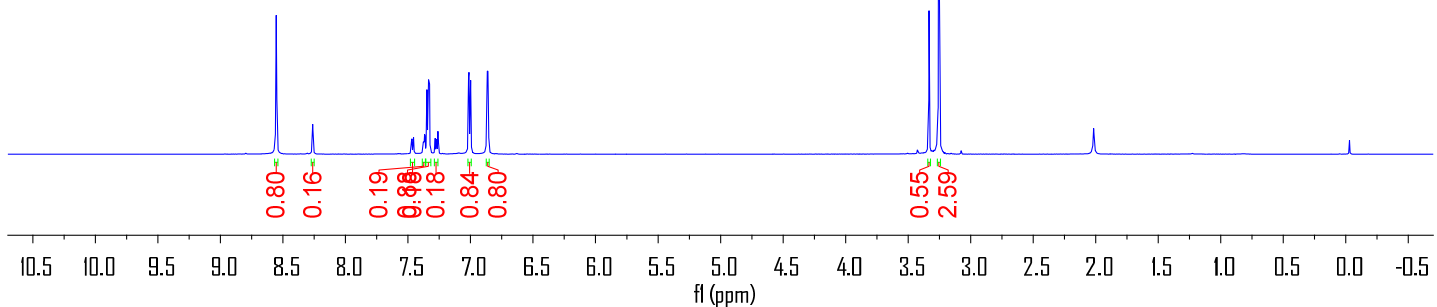



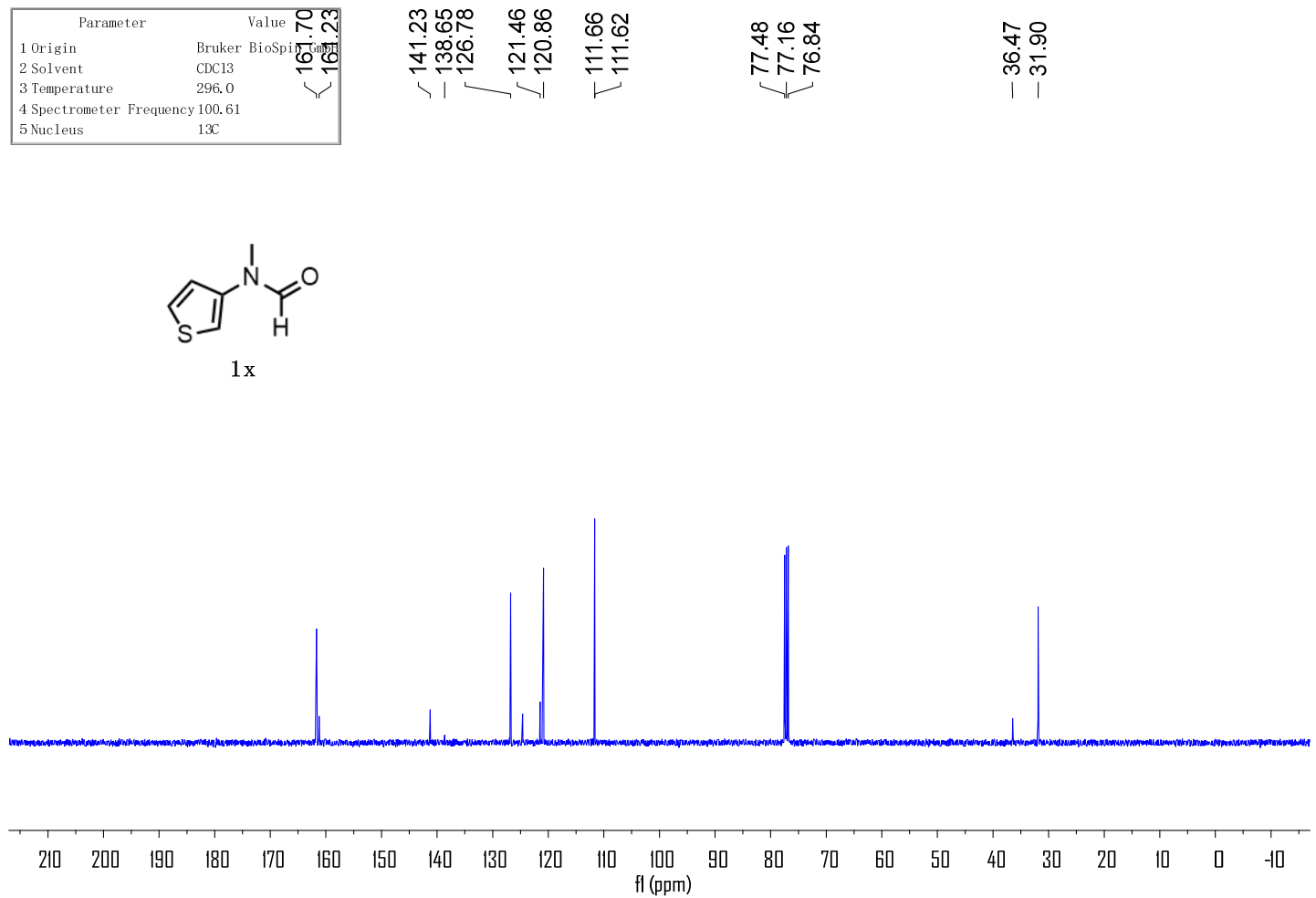

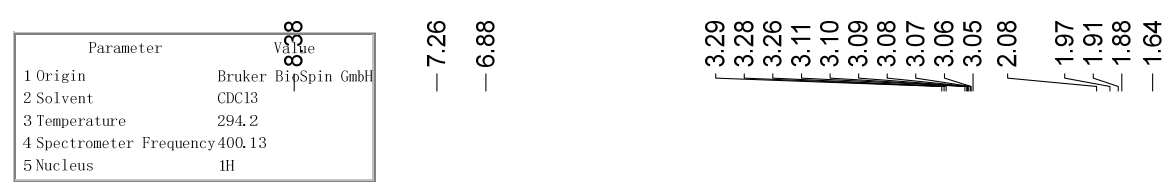

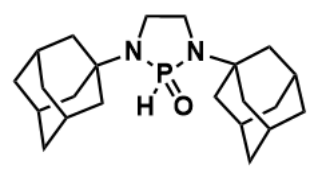

$\mathrm{L}^{12}$

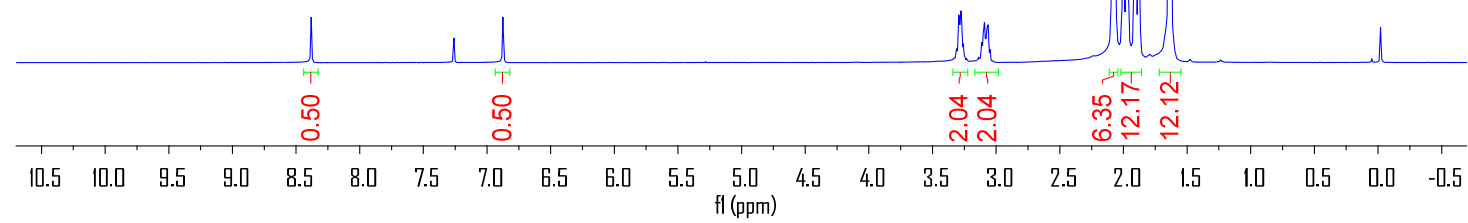



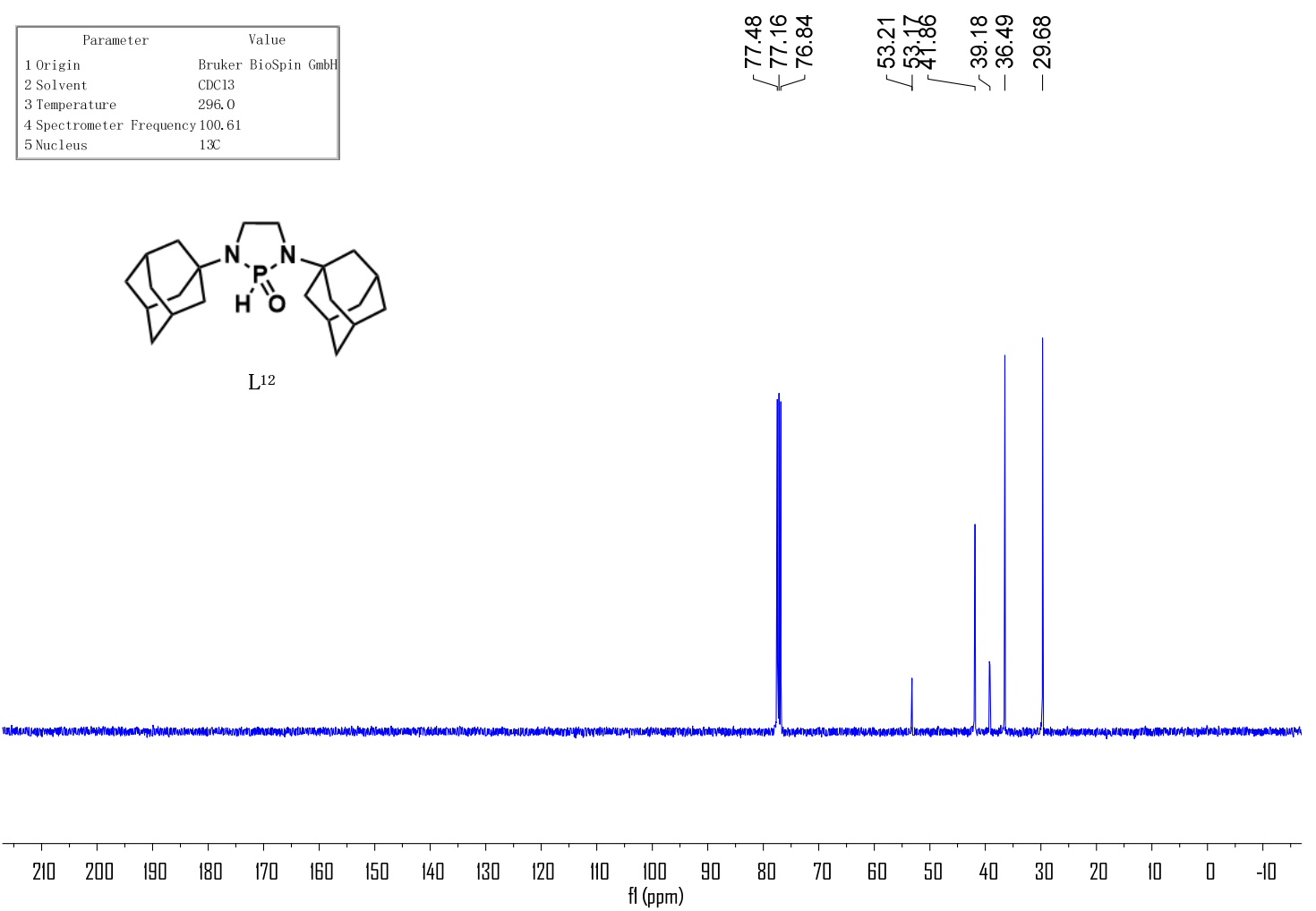

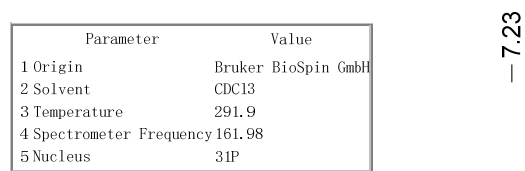

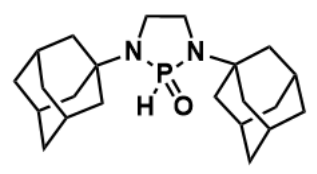

$\mathrm{L}^{12}$

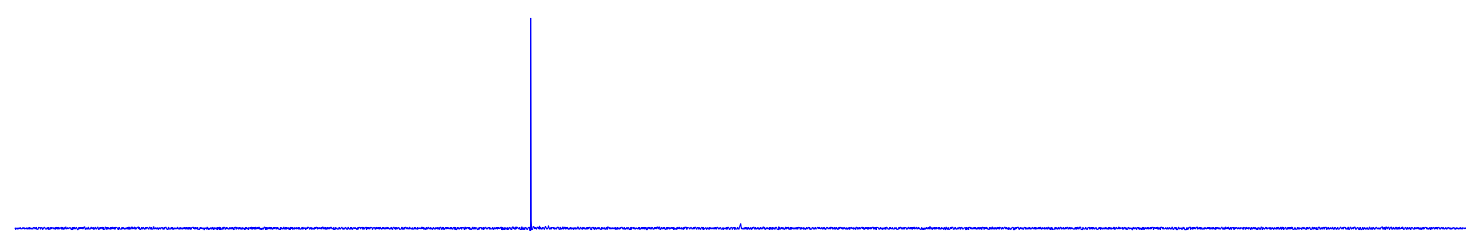

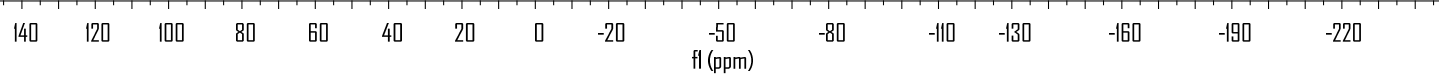




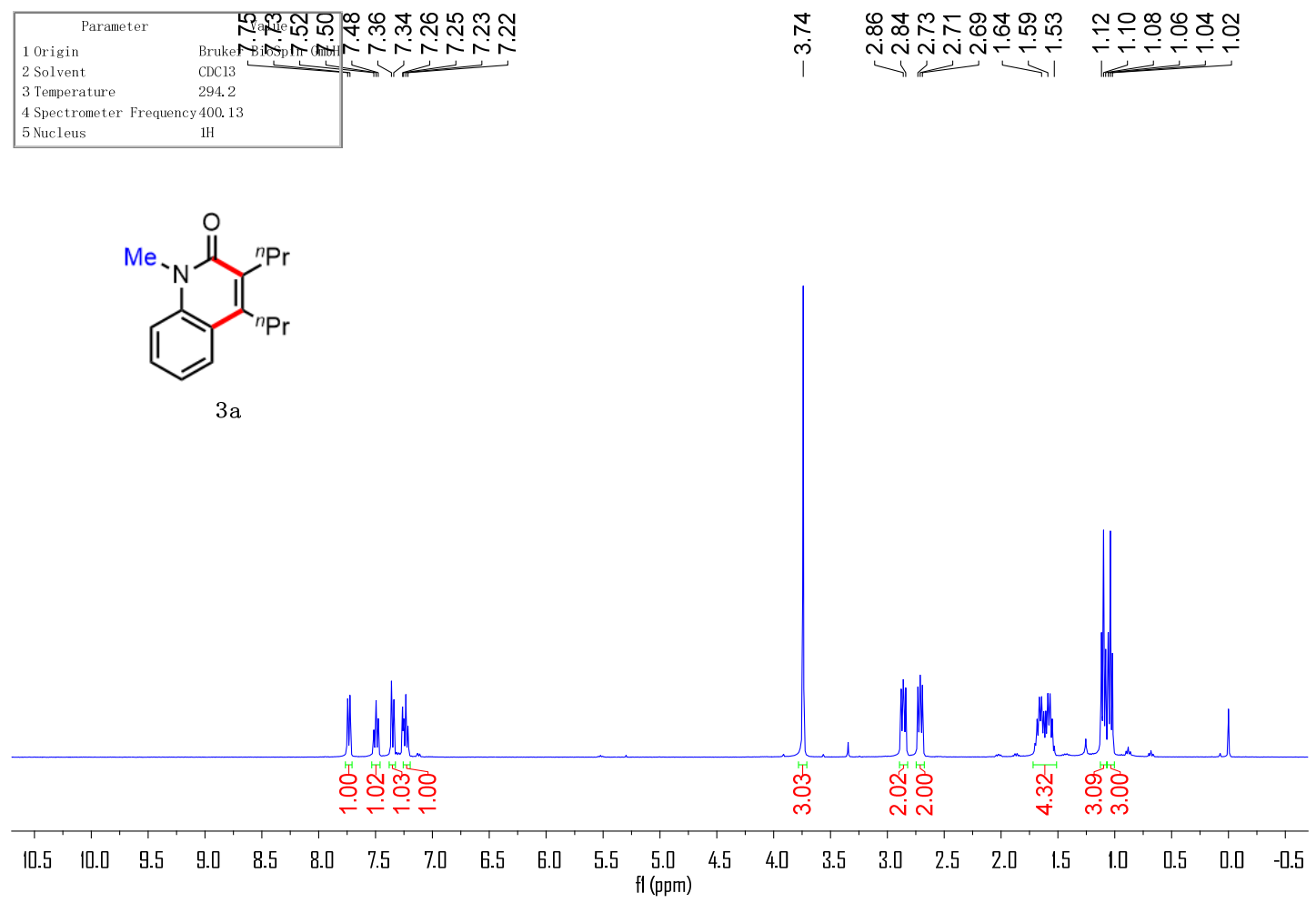

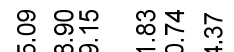
守 난

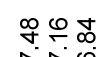

Nก๊
중ำ 요 웅

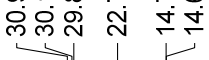

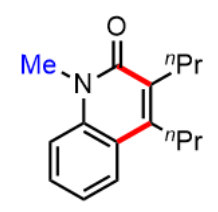

$3 a$

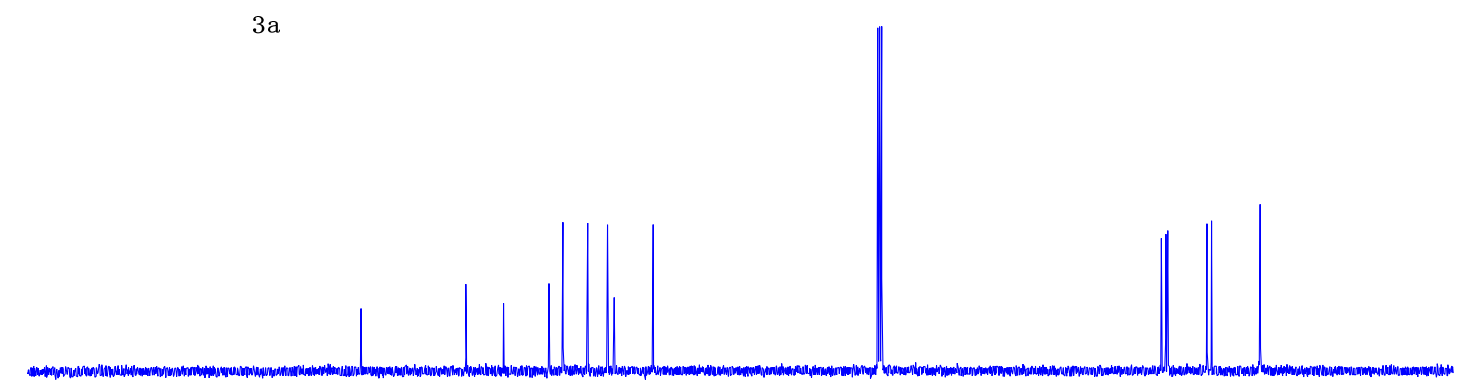

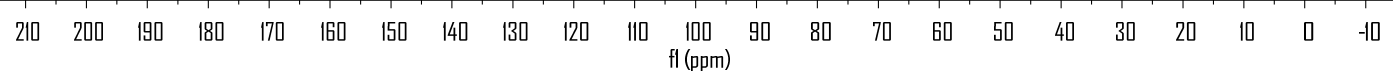




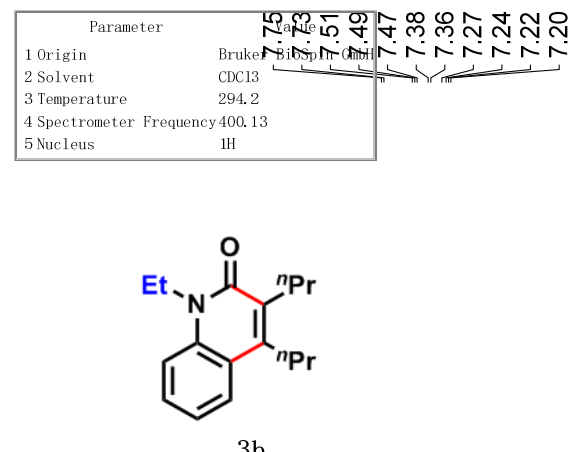

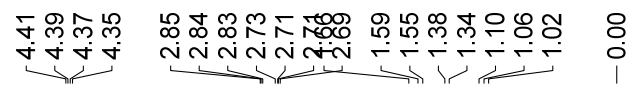

$3 \mathrm{~b}$

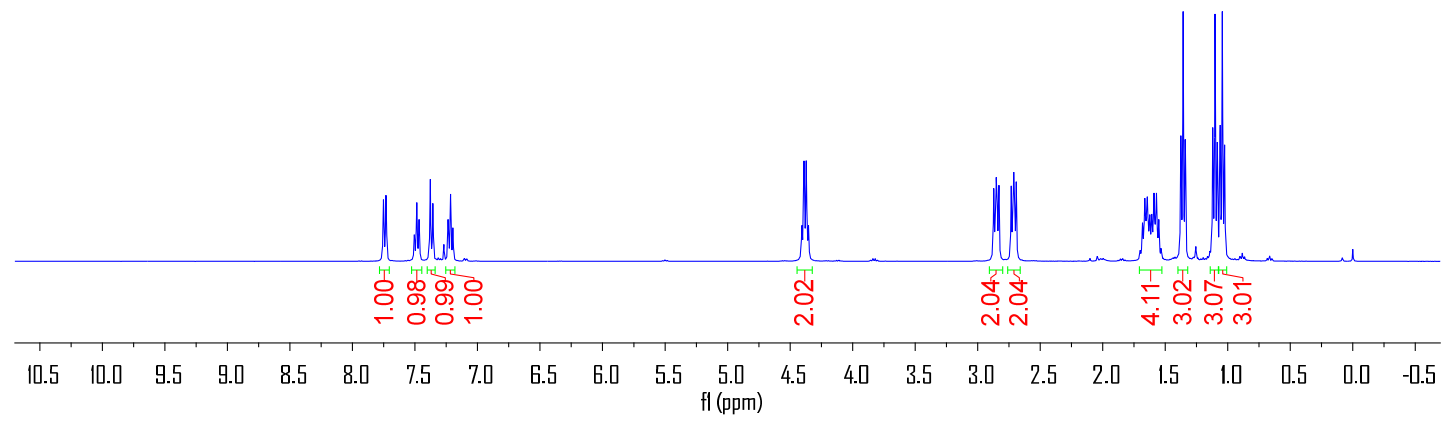

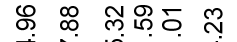

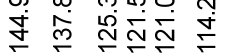

$\frac{1}{2} \sqrt{-1}$

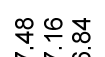

상

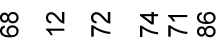

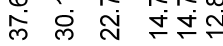

$\begin{array}{lr}2 \text { Solvent } & \text { CDC13 } \\ 3 \text { Temperature } & 296.0 \\ 4 \text { Spectrometer } & \text { Frequency } 100.61\end{array}$

5 Nucleus $13 \mathrm{C}$

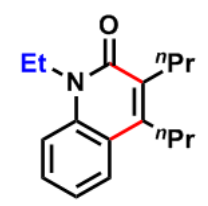

$3 b$

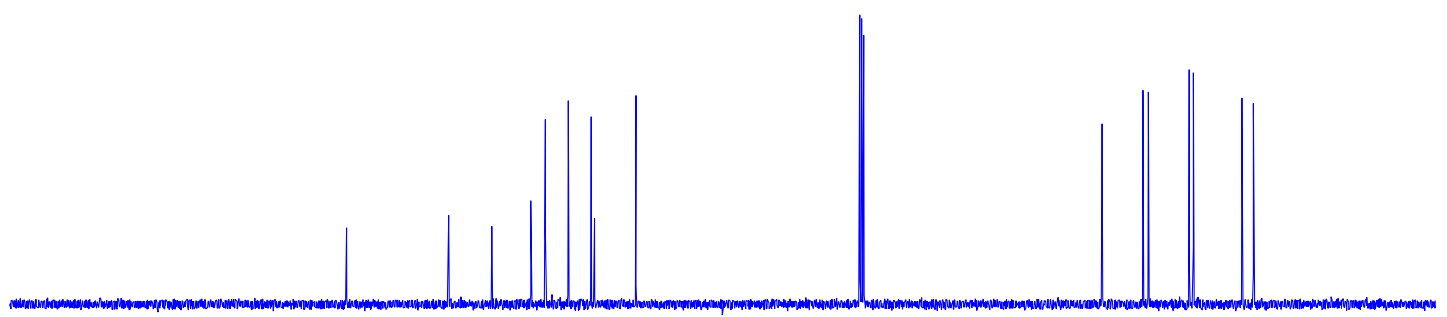

$\begin{array}{lllllllllllllllllllllllll}210 & 200 & 190 & 180 & 170 & 160 & 150 & 140 & 130 & 120 & 110 & 100 & 90 & 80 & 70 & 60 & 50 & 40 & 30 & 20 & 10 & 0 & -10\end{array}$ 

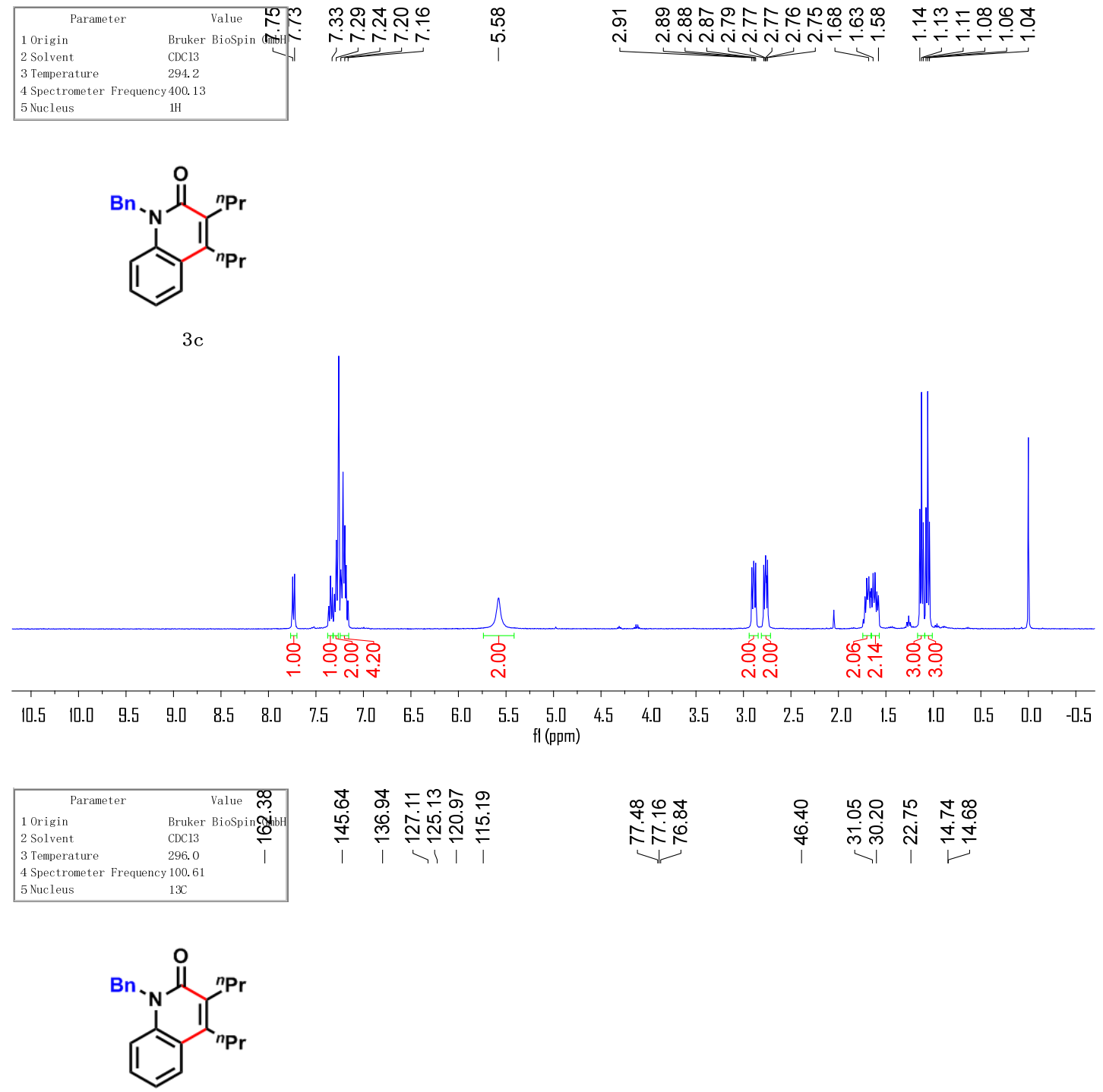

$3 \mathrm{c}$

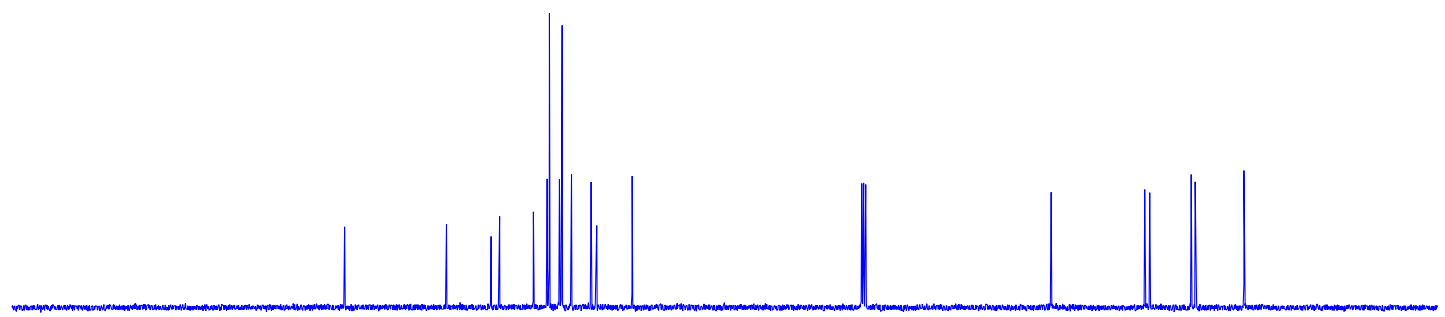

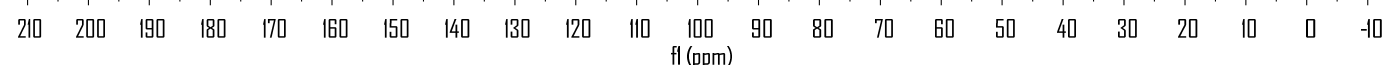




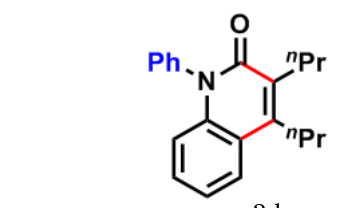

$3 d$

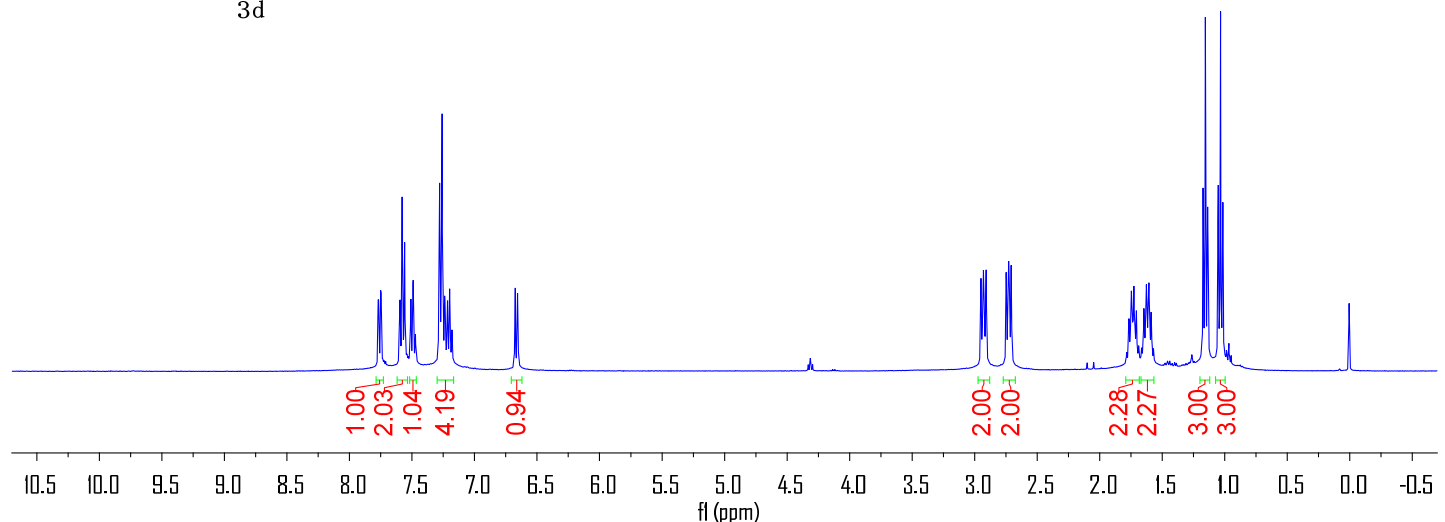

ลิ ณำ ㅇํำํำ

守

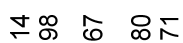

க்

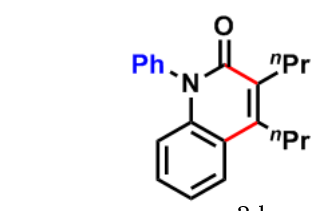

$3 d$

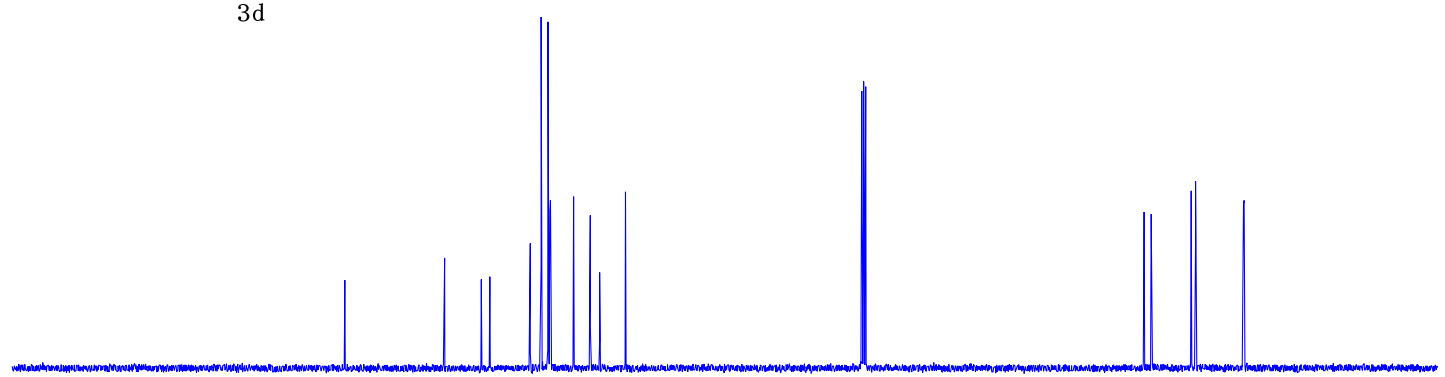

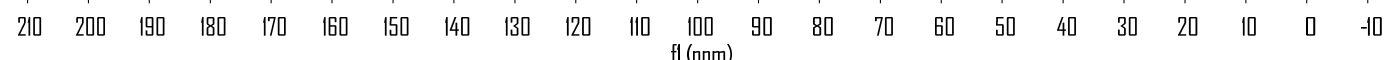




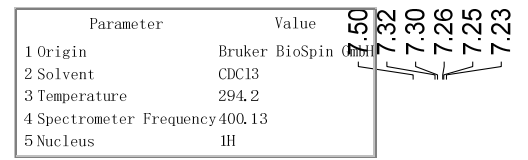

$3 \mathrm{e}$

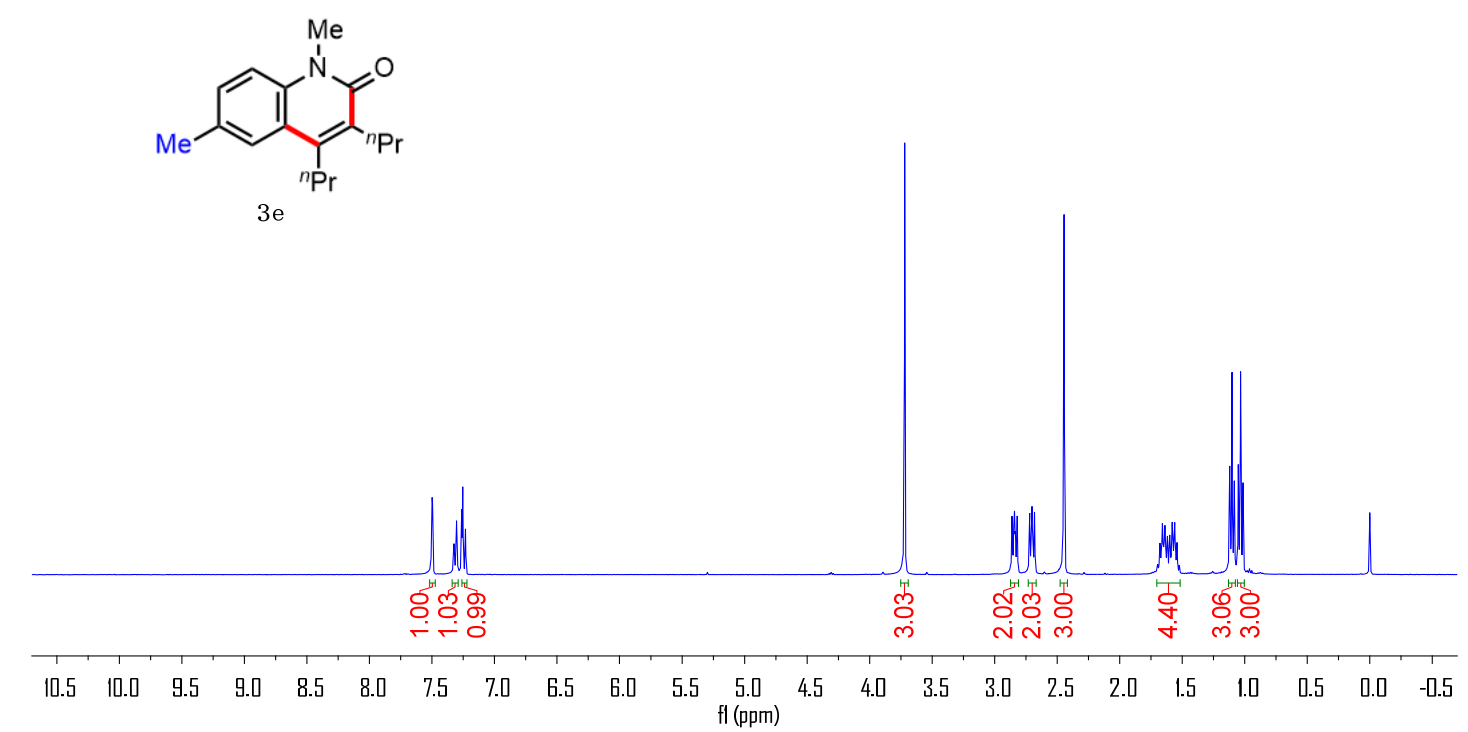

신

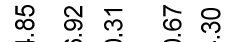

过

$\stackrel{i}{i} \stackrel{\text { I }}{\leftarrow}$

296.0

5 Nucleus $\quad 13 \mathrm{C}$
$3 e$

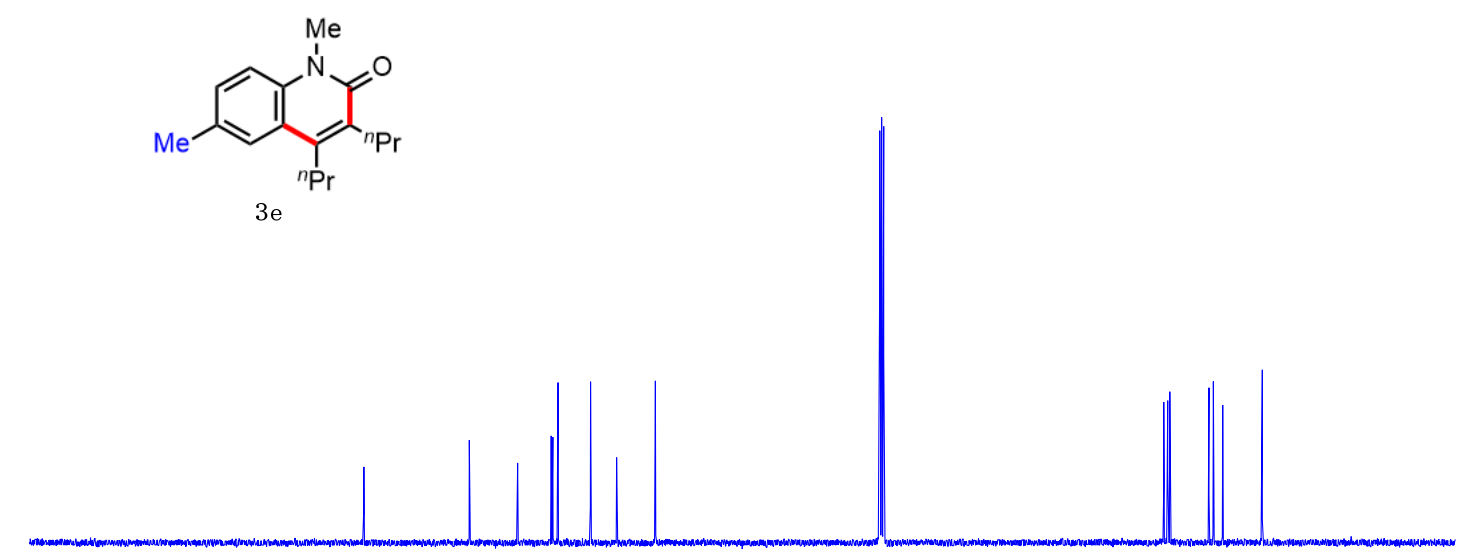

$\stackrel{\infty}{\infty} \div$

ペN゚

rin

องิ

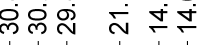

$\begin{array}{llllllllllllllllllllllll}210 & 200 & 190 & 180 & 170 & 160 & 150 & 140 & 130 & 120 & 110 & 100 & 90 & 80 & 70 & 60 & 50 & 40 & 30 & 20 & 10 & 0 & -10\end{array}$ 


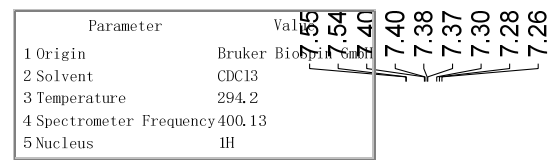

$3 f$

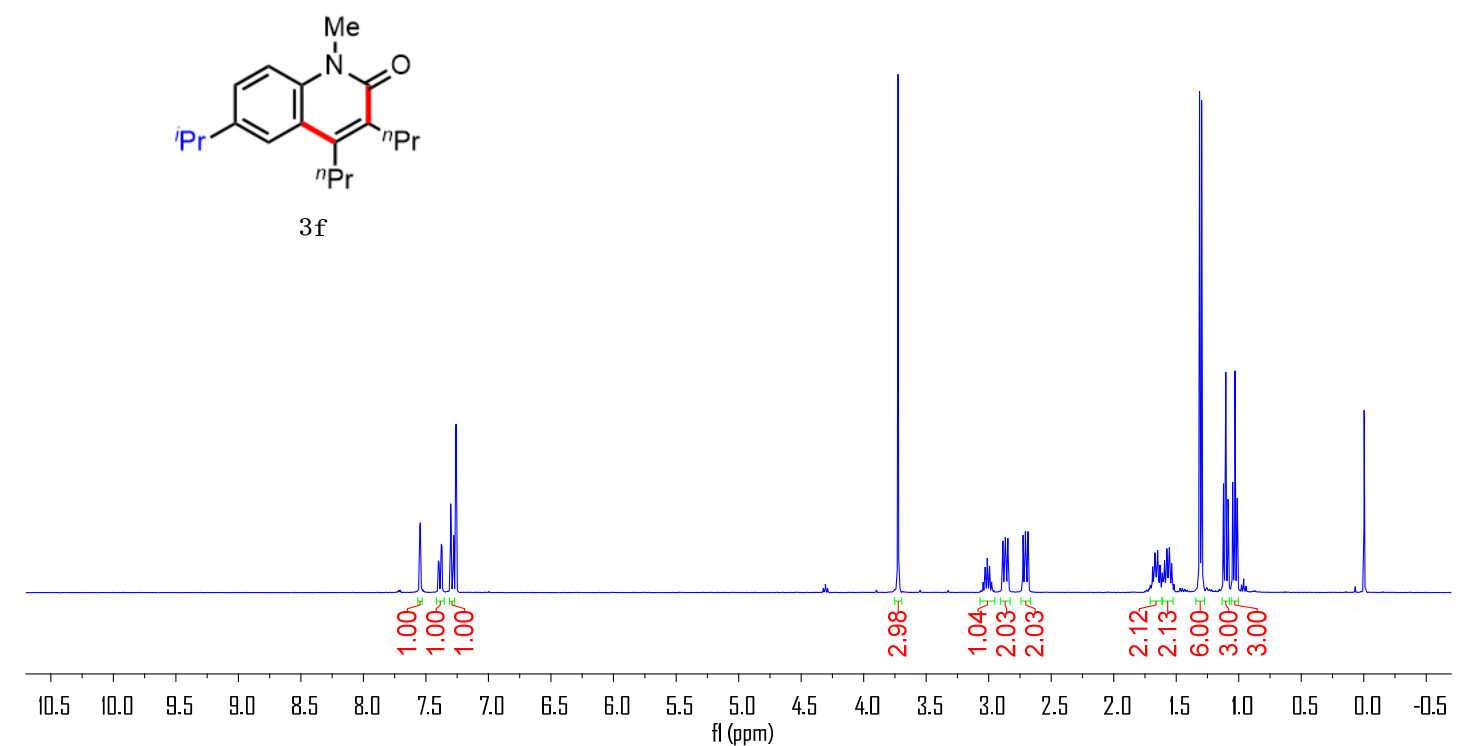

గ్

min NiNiN Ni r
ธָกับ

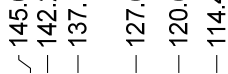

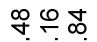
손
-

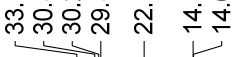

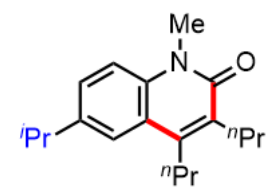

$3 f$

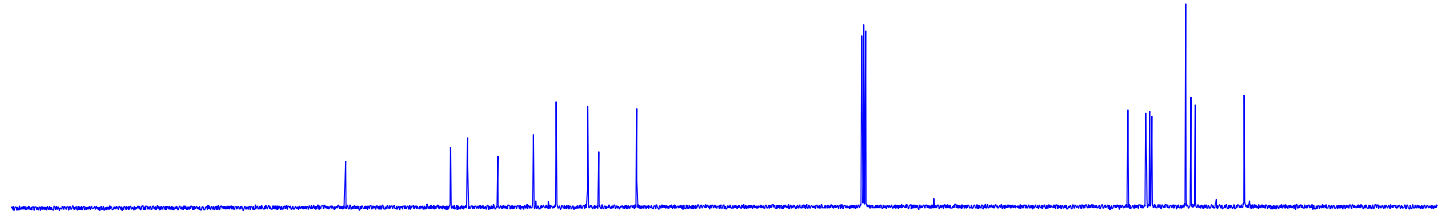

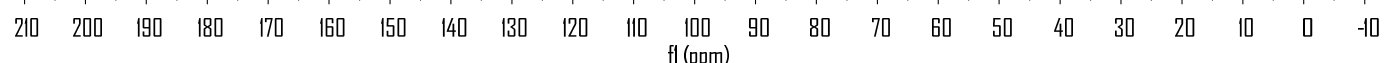




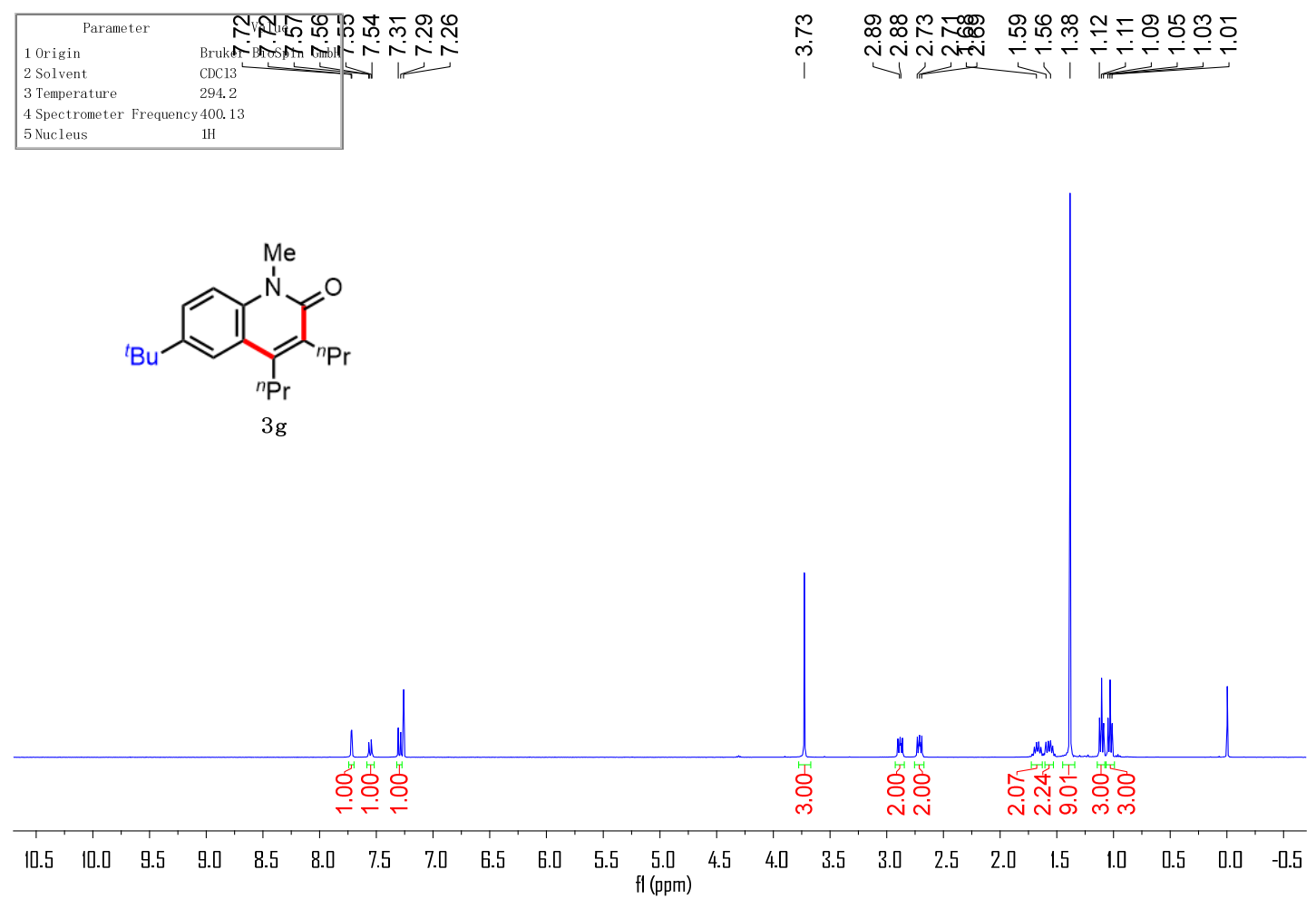

\begin{tabular}{|c|c|c|c|c|c|c|}
\hline Parameter & 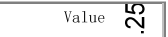 & œ워 & 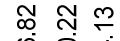 & 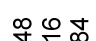 & পণ্் & ח \\
\hline $\begin{array}{l}1 \text { Origin } \\
2 \text { Solvent }\end{array}$ & $\begin{array}{l}\text { Bruker BioSpin तुं } \\
\text { CDCl3 }\end{array}$ & 守 & 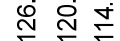 & N实禹 & 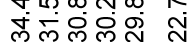 & $\underset{\leftarrow}{\leftarrow}$ \\
\hline 3 Temperature & 296.0 & i & i & 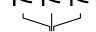 & 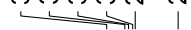 & \\
\hline 4 Spectrometer Frequenc & y 100.61 & & & & & \\
\hline 5 Nucleus & $13 \mathrm{C}$ & & & & & \\
\hline
\end{tabular}

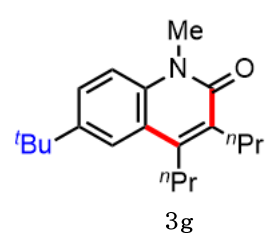

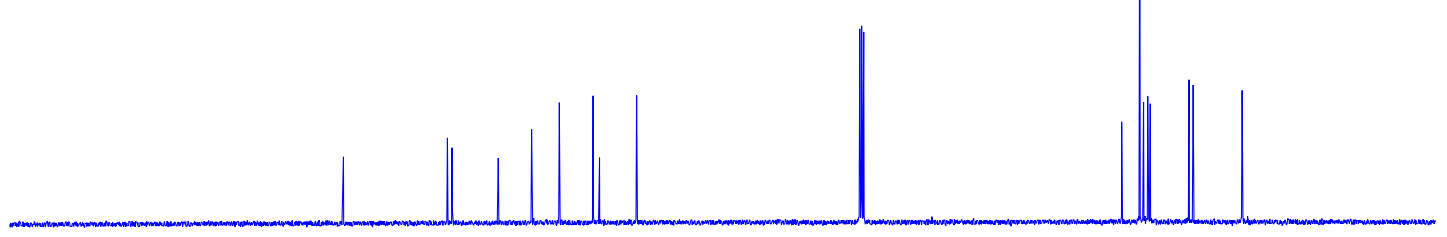

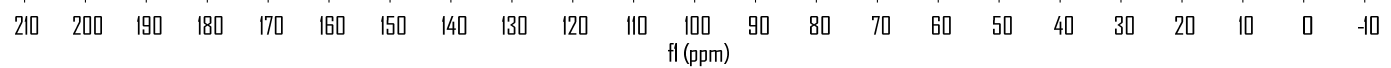




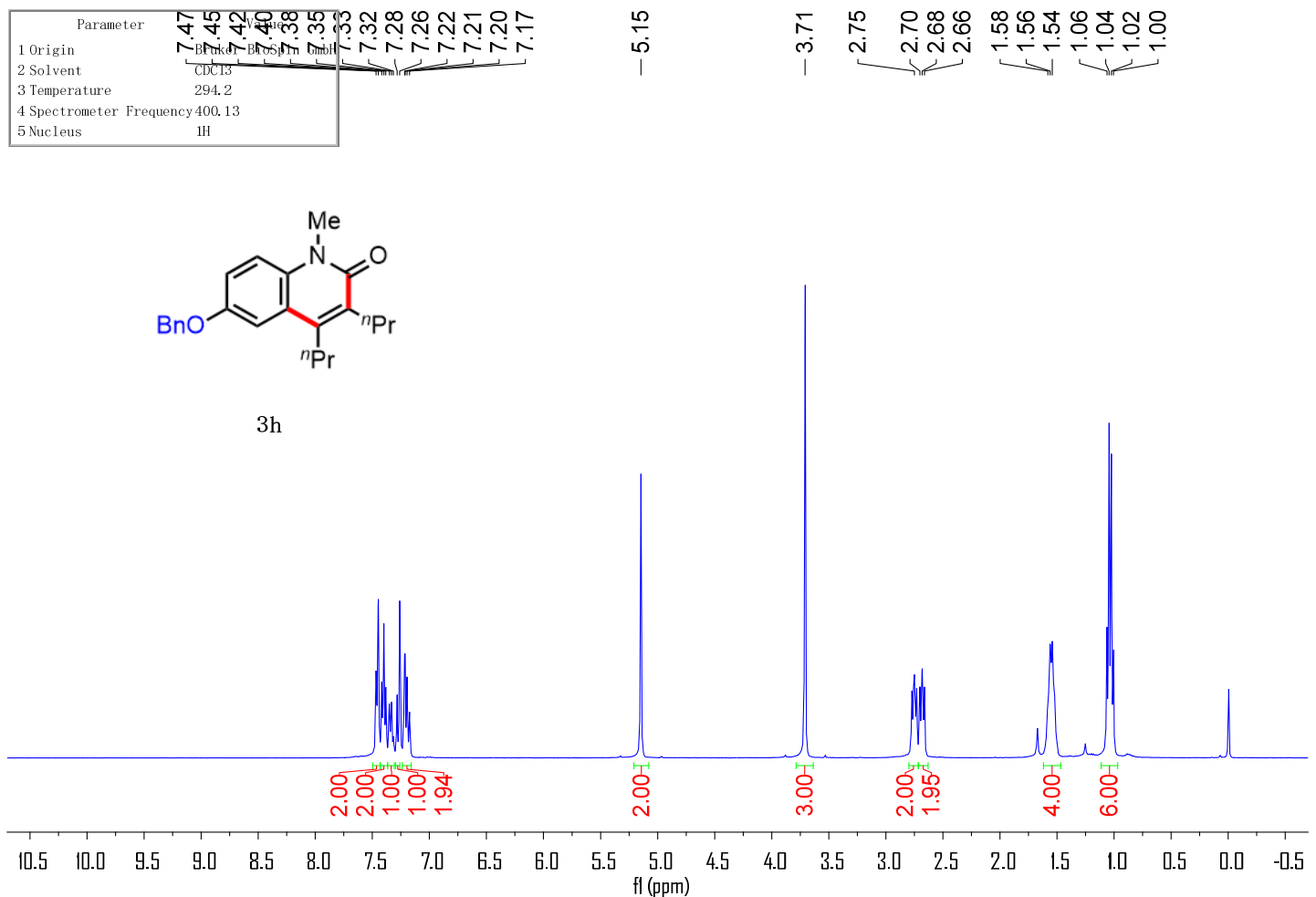

Parameter Value $\infty$ 요

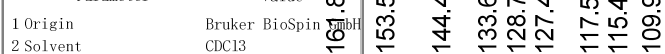

3 Temperature 296.0

4 Spectrometer Frequency 100.6
5 Nucleus
$13 \mathrm{C}$

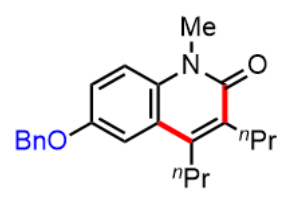

$3 \mathrm{~h}$

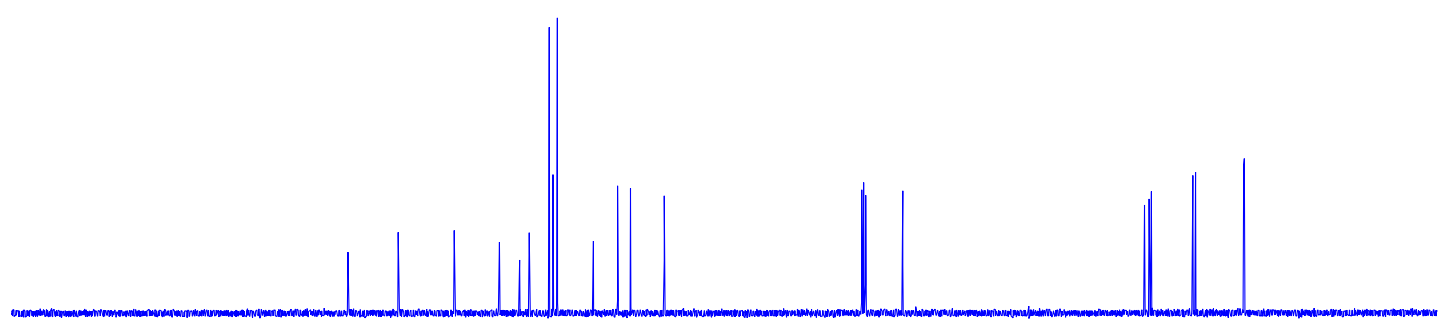

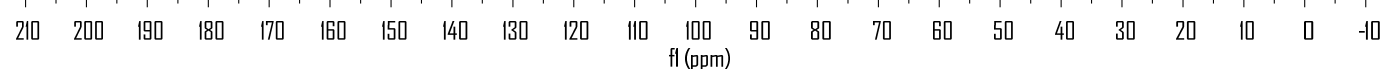




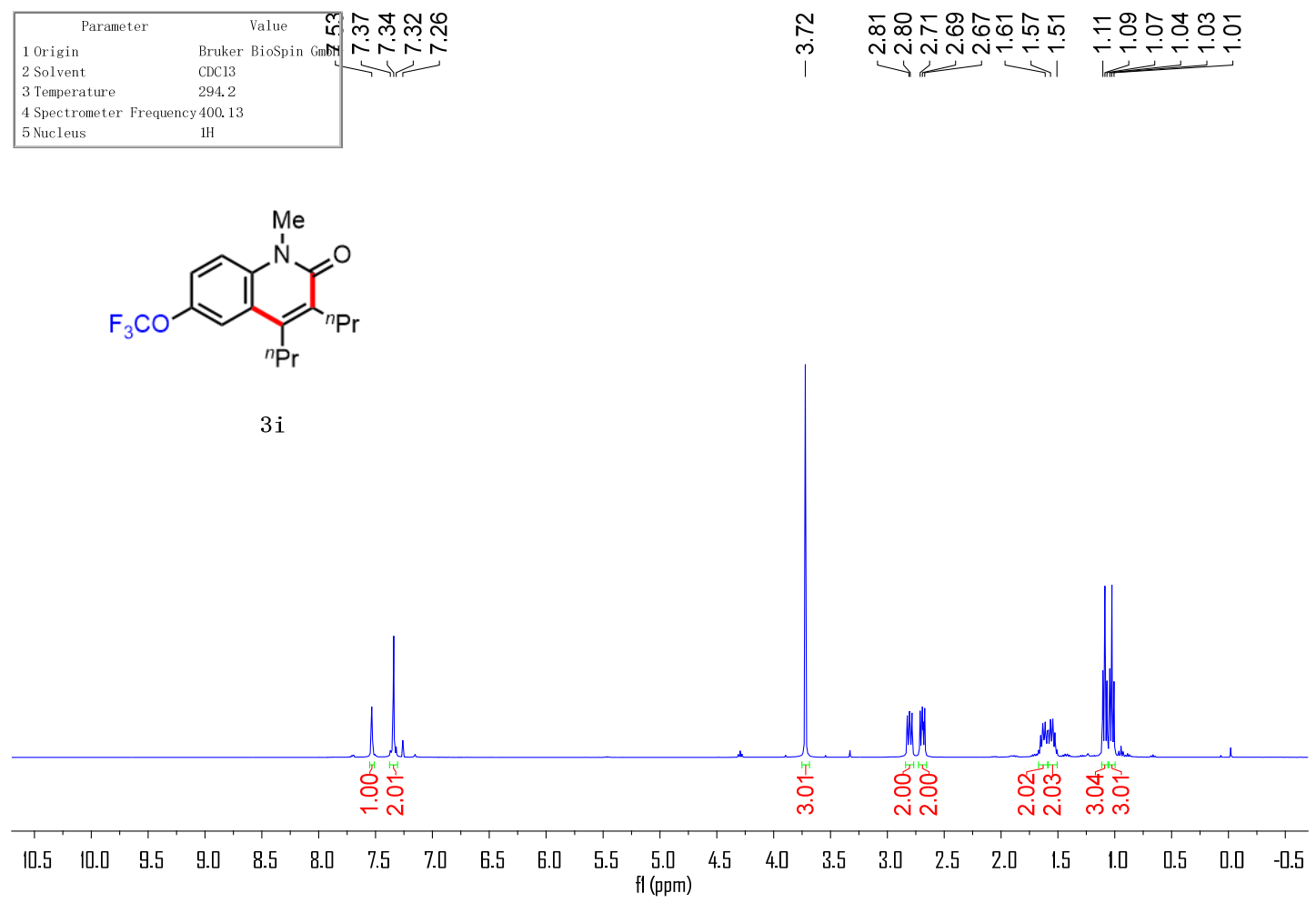

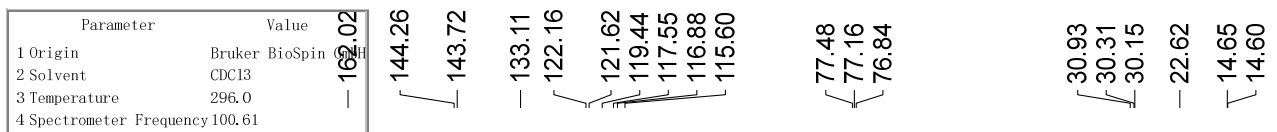

4 Spectrometer Frequency 100.6
5 Nucleus
$13 \mathrm{C}$

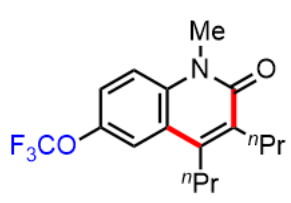

$3 i$

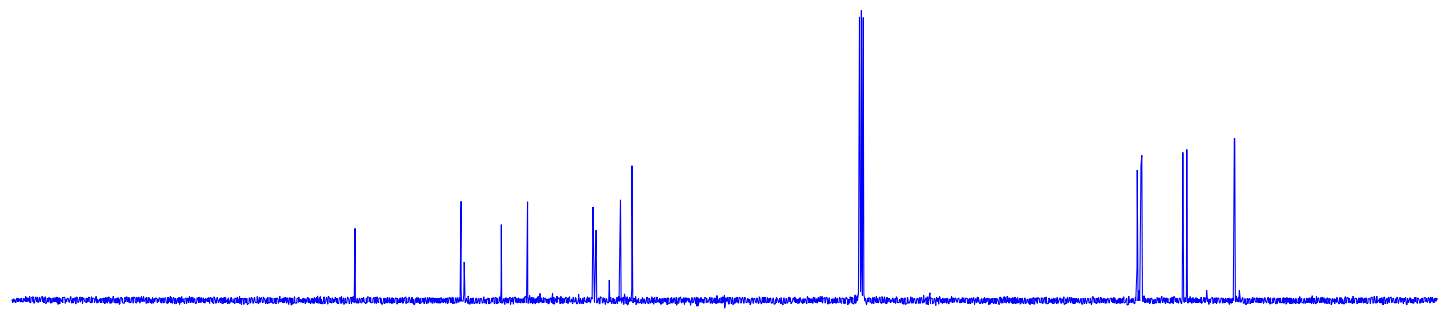

$\begin{array}{llllllllllllllllllllllllllll}210 & 200 & 190 & 180 & 170 & 160 & 150 & 140 & 130 & 120 & 110 & 100 & 50 & 80 & 70 & 60 & 50 & 40 & 30 & 20 & 10 & 0 & -10\end{array}$ 

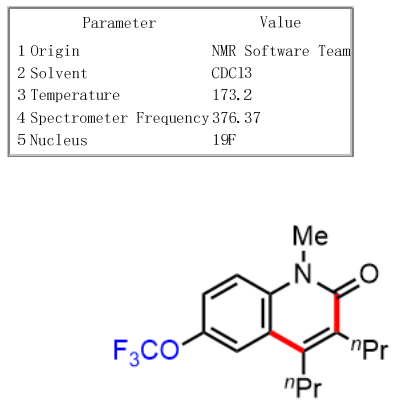

$3 i$

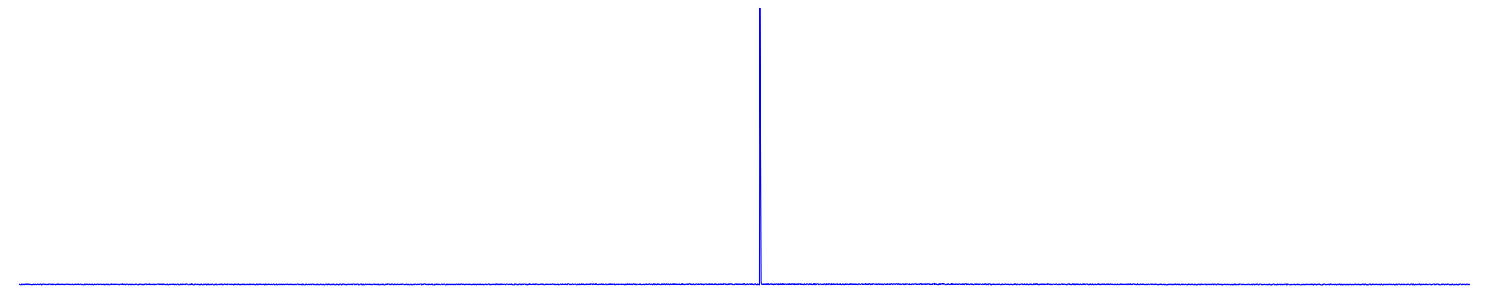

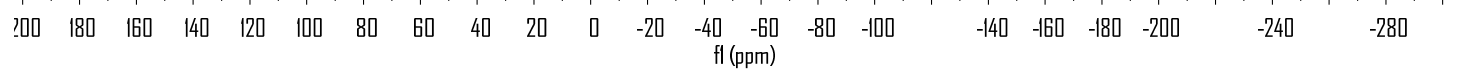

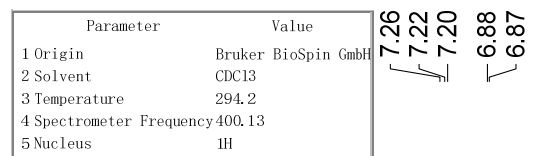

r d

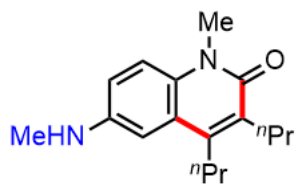

$3 \mathrm{j}$

i

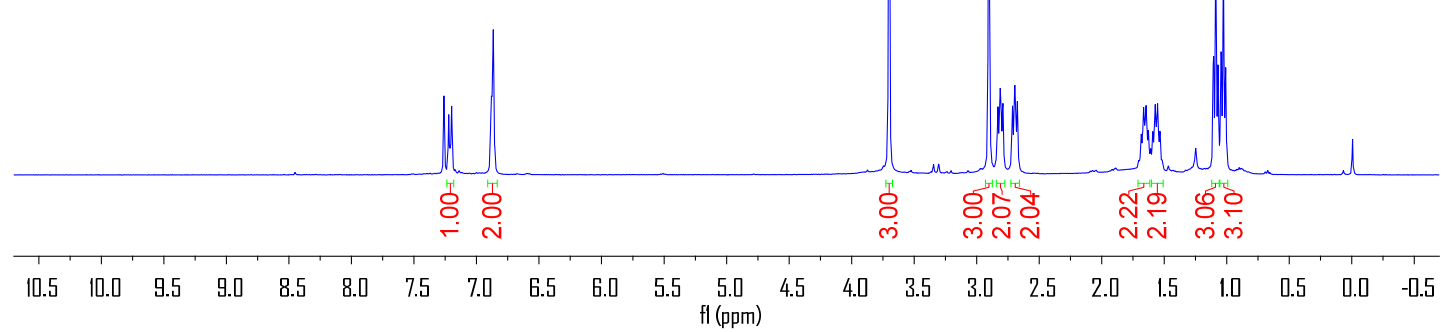




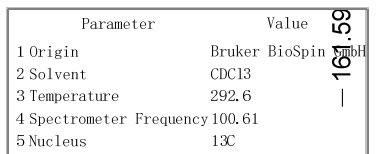

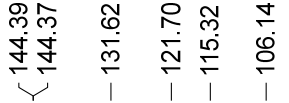

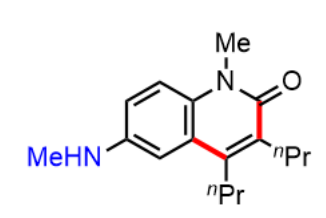

$3 \mathrm{j}$

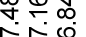

소

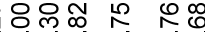

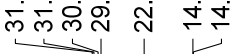

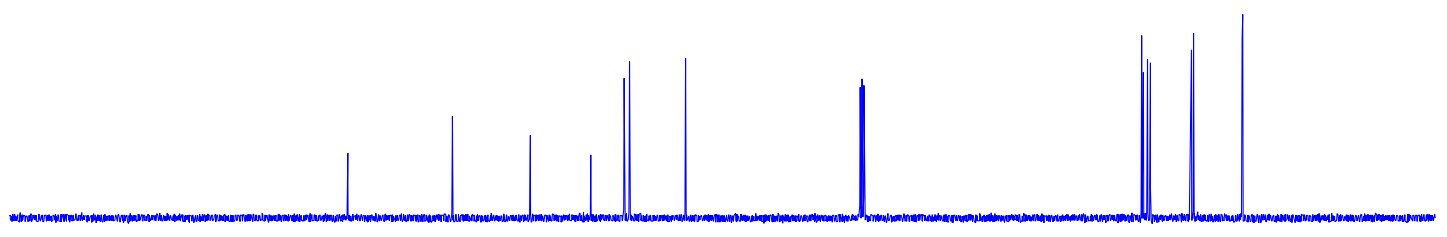

$\begin{array}{lllllllllllllllllllllllll}210 & 200 & 190 & 180 & 170 & 160 & 150 & 140 & 130 & 120 & 110 & 100 & 90 & 80 & 70 & 60 & 50 & 40 & 30 & 20 & 10 & 0 & -10\end{array}$

\begin{tabular}{|lc|}
\hline \multicolumn{1}{|c|}{ Parameter } & Value \\
1 Origin & Bruker BioSpin GmbH \\
2 Solvent & CDCl3 \\
3 Temperature & 294.2 \\
4 Spectrometer Frequency 400.13 & \\
5 Nucleus & 1H
\end{tabular}

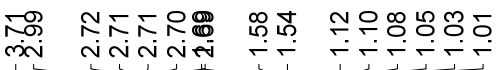

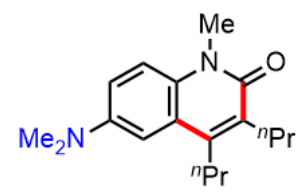

$3 \mathrm{k}$

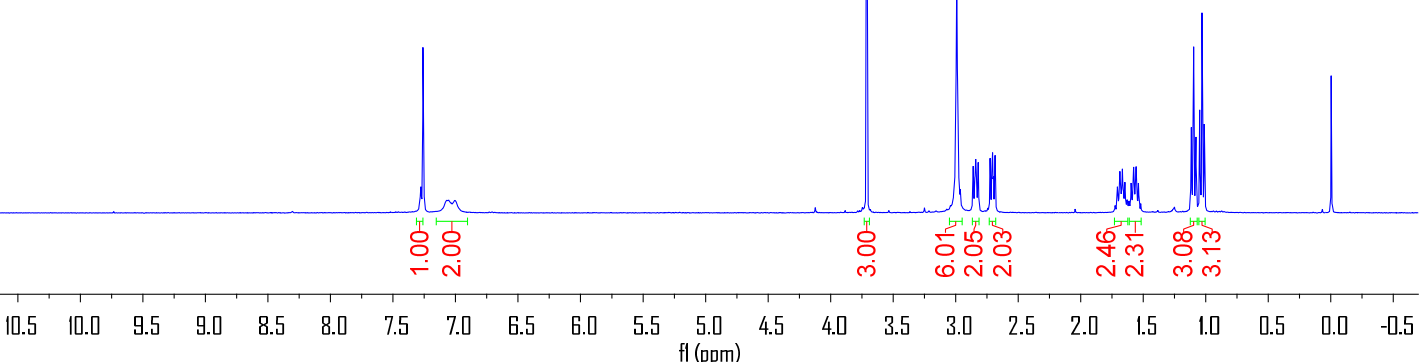




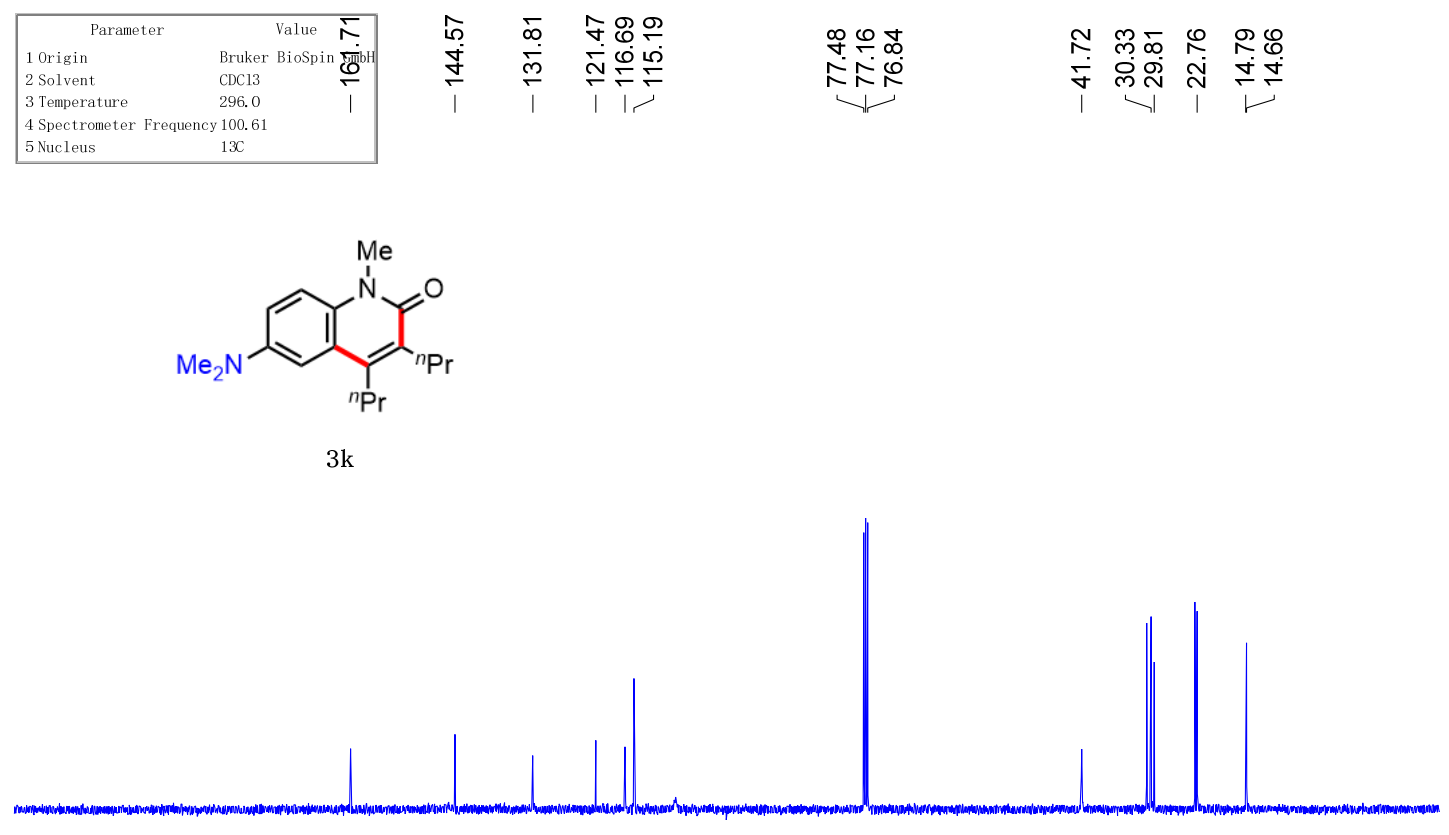

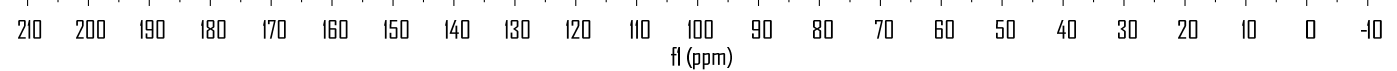

Parameter
1 Origin
2 Solvent
3 Temperature
4 Spectrometer Frequency 400.13
5 Nucleus
1H

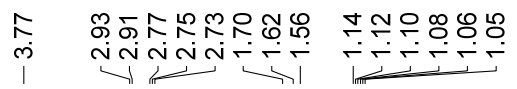

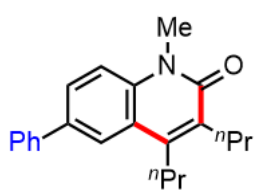

31

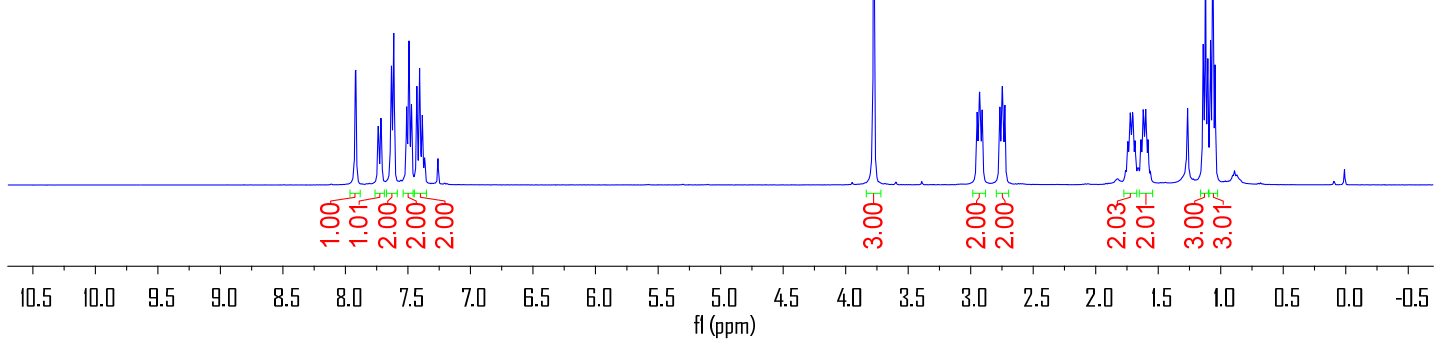




\begin{tabular}{|ll|}
\hline \multicolumn{1}{|c|}{ Parameter } & Value \\
1 Origin & Bruker BioSpin \\
2 Solvent & CDC13 \\
3 Temperature & 296.0 \\
4 Spectrometer Frequency 100.61 \\
5 Nucleus & $13 \mathrm{C}$ \\
\hline
\end{tabular}

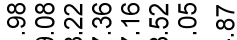

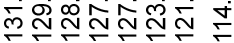

5 - 130<smiles>CCCc1c(C(C)C)c2cc(-c3ccccc3)ccc2n(C)c1=O</smiles>

31

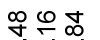

Кト゚

II

m요용

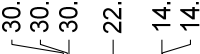

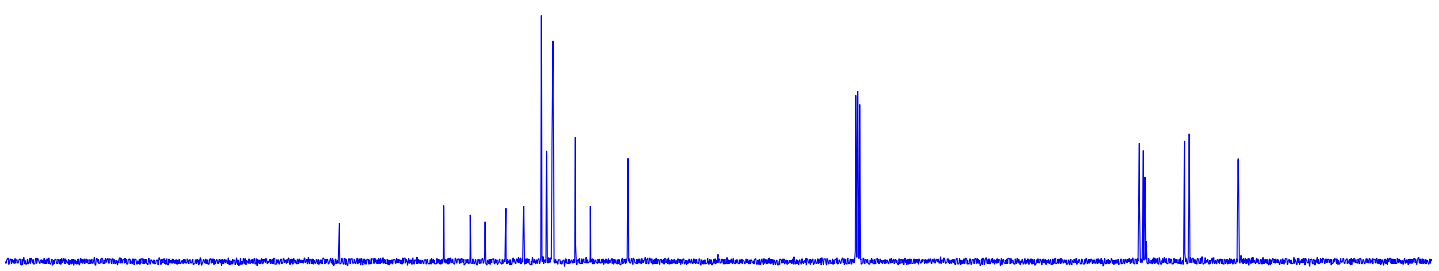

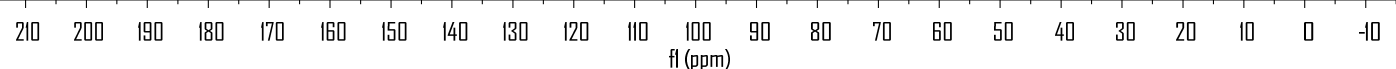

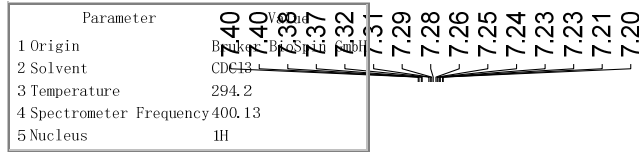

皇<smiles>CCCc1c(C(C)C)c2cc(F)ccc2n(C)c1=O</smiles>

$3 \mathrm{~m}$

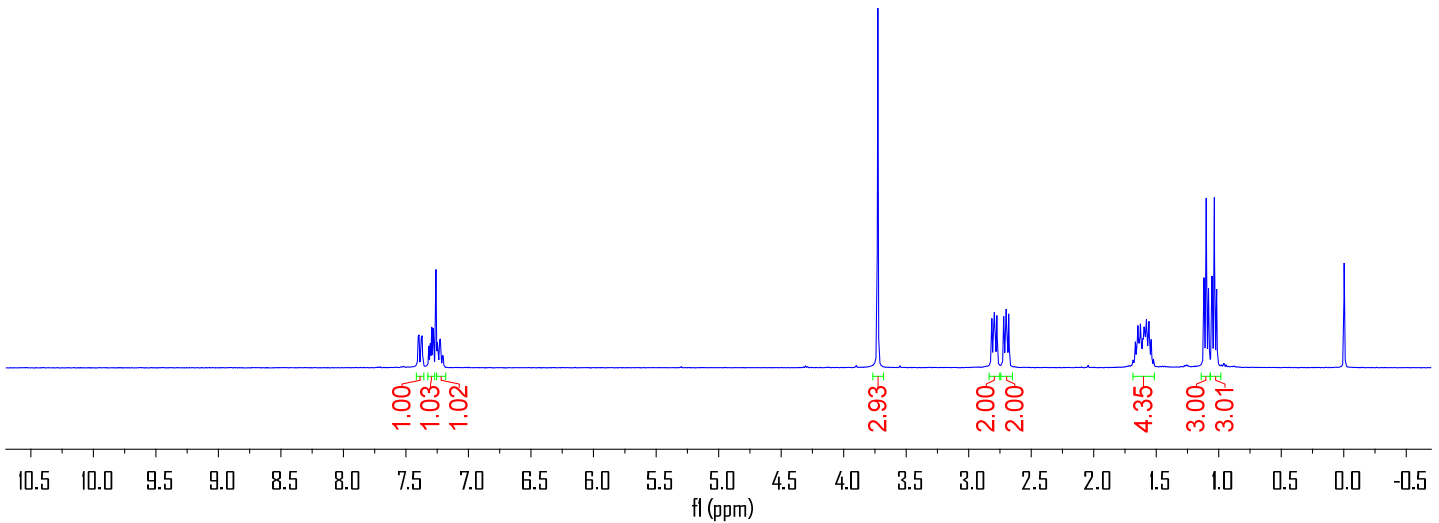




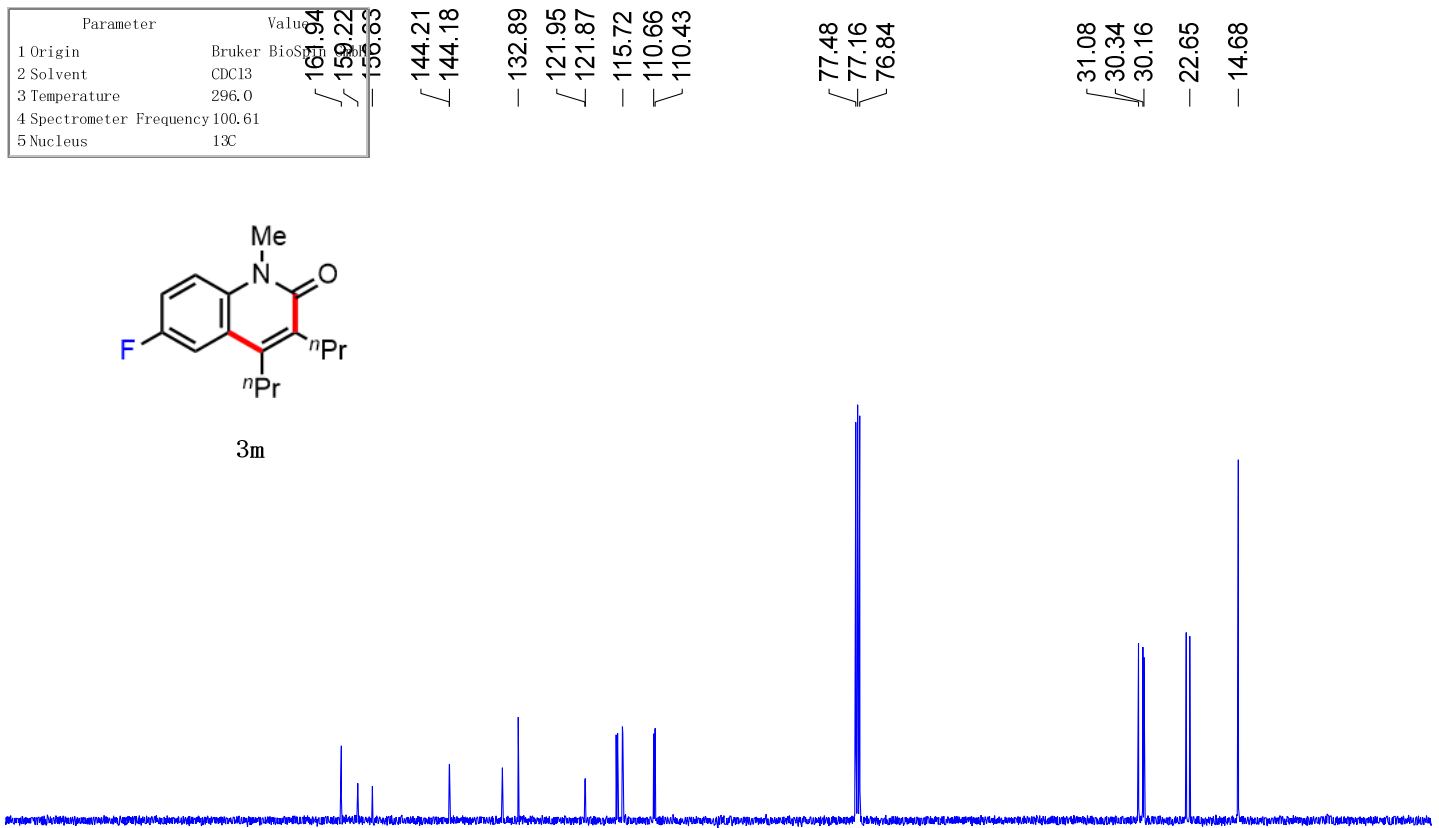

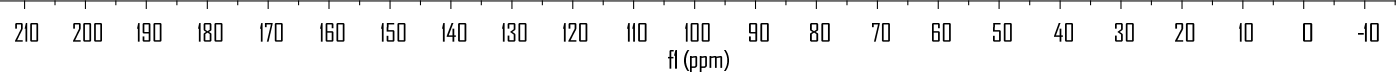

\begin{tabular}{|lc|}
\hline \multicolumn{1}{|c|}{ Parameter } & \multicolumn{1}{c|}{ Value } \\
1 Origin & NMR Software Team \\
2 Solvent & CDC13 \\
3 Temperature & 173.2 \\
4 Spectrometer & Frequency 376.37 \\
5 Nucleus & 19F \\
\hline
\end{tabular}

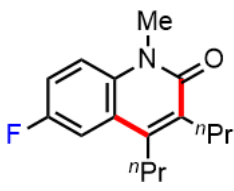

$3 \mathrm{~m}$

$\begin{array}{lllllllllllllllllllllllllllllll}70 & 60 & 50 & 40 & 30 & 20 & 10 & 0 & -10 & -20 & -30 & -40 & -50 & -60 & -70 & -80 & -90 & -100 & -110 & & -130 & -140 & -150 & -160 & -170 & -180\end{array}$ 


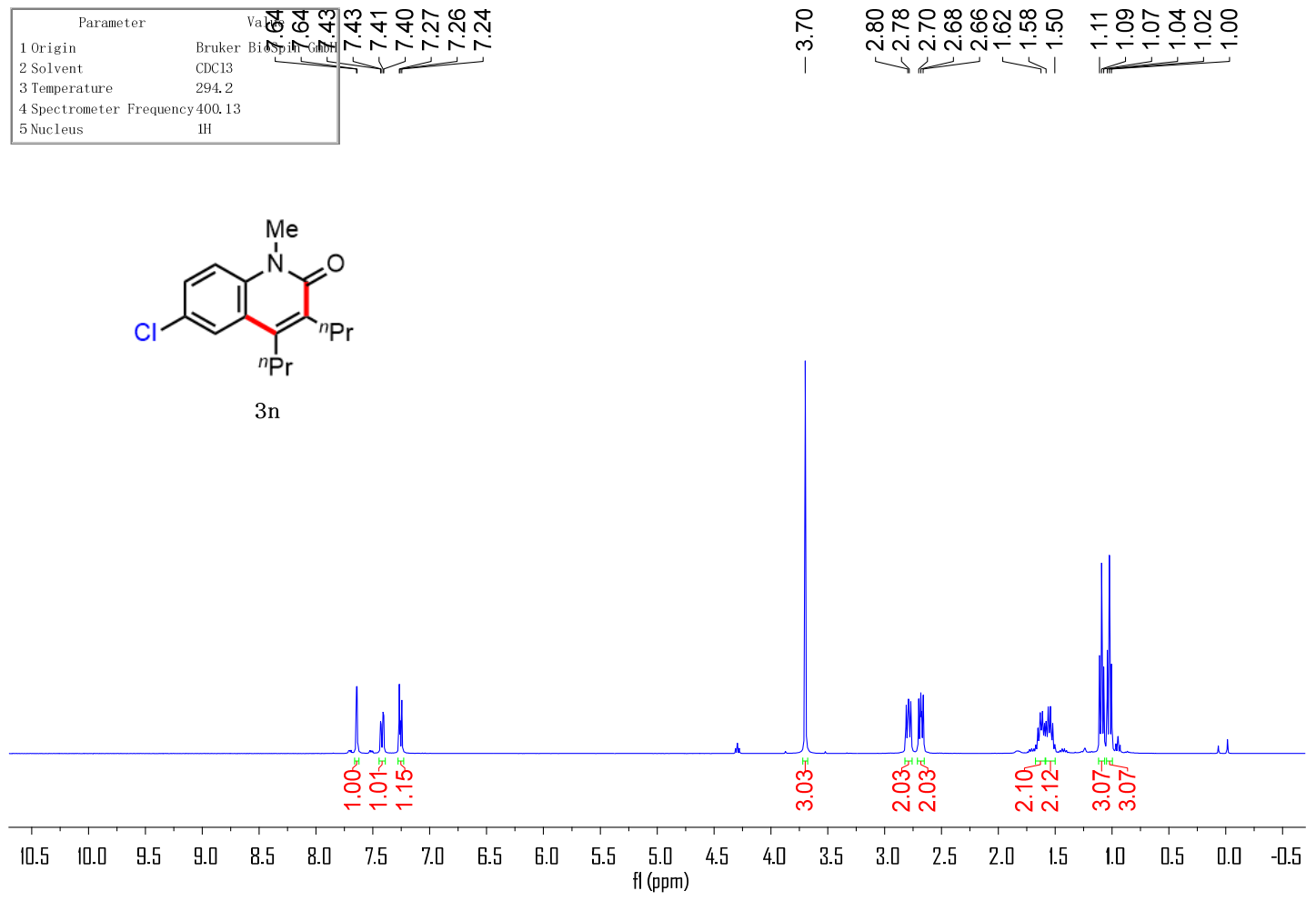

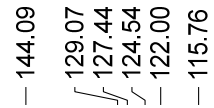

$\begin{array}{ll}\text { 2 Solvent } & \text { Bruker BioSpin } \\ 3 \text { Temperature } & 296.0\end{array}$

4 Spectrometer Frequency 100.61

$13 \mathrm{C}$

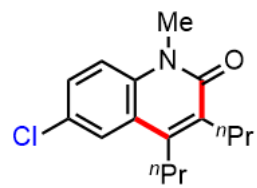

$3 n$

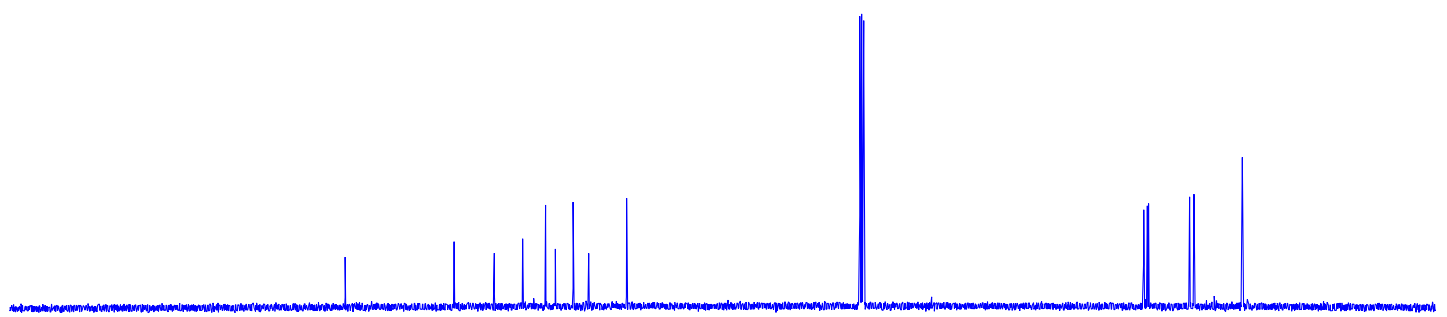

$\begin{array}{llllllllllllllllllllllll}210 & 200 & 190 & 180 & 170 & 160 & 150 & 140 & 130 & 120 & 110 & 100 & 90 & 80 & 70 & 60 & 50 & 40 & 30 & 20 & 10 & 0 & -10\end{array}$ 

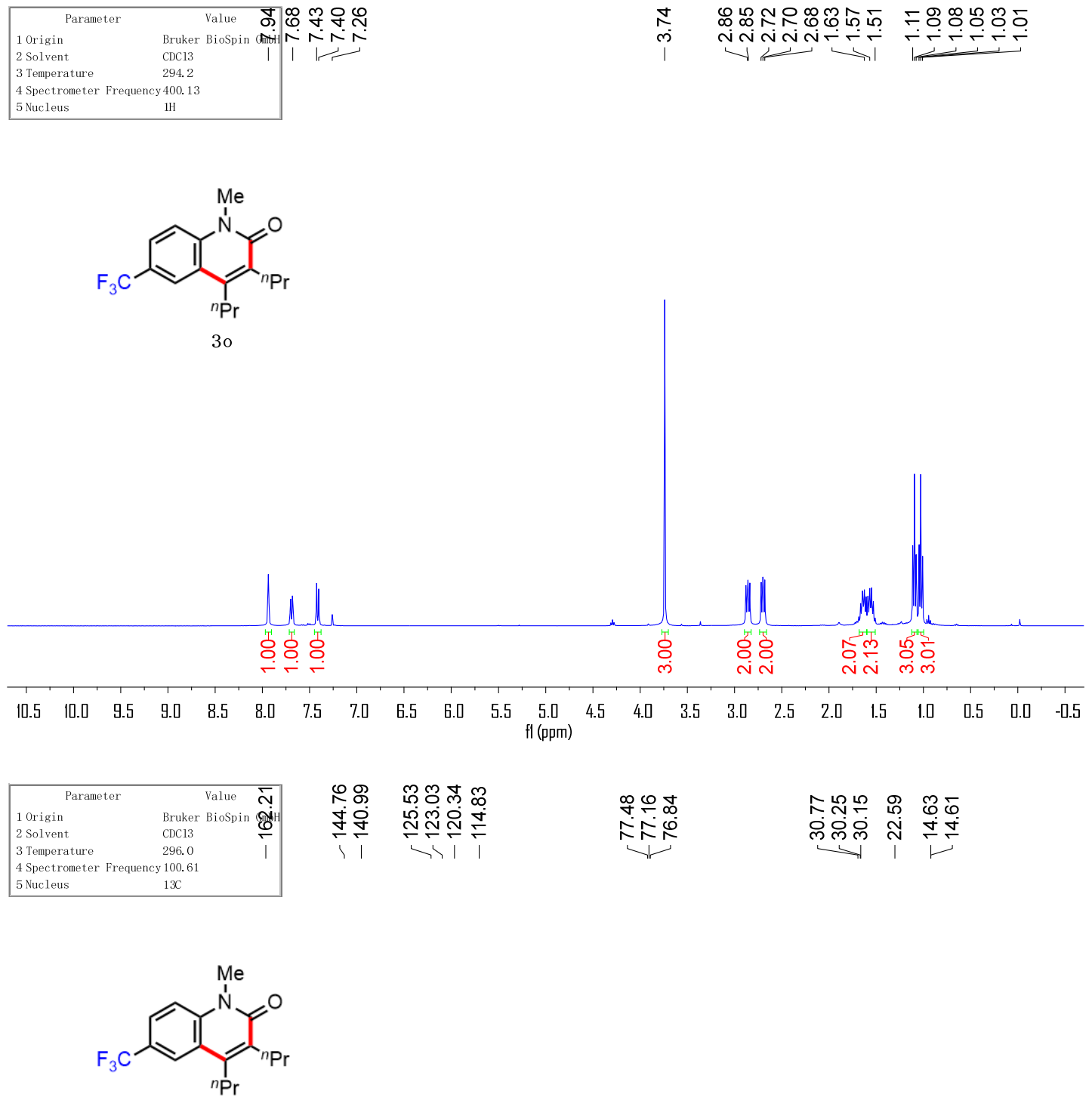

용 ஜூయ

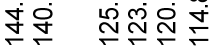

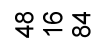

사뉴

슈ํำ 용

I। पi।

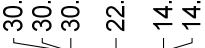

3o

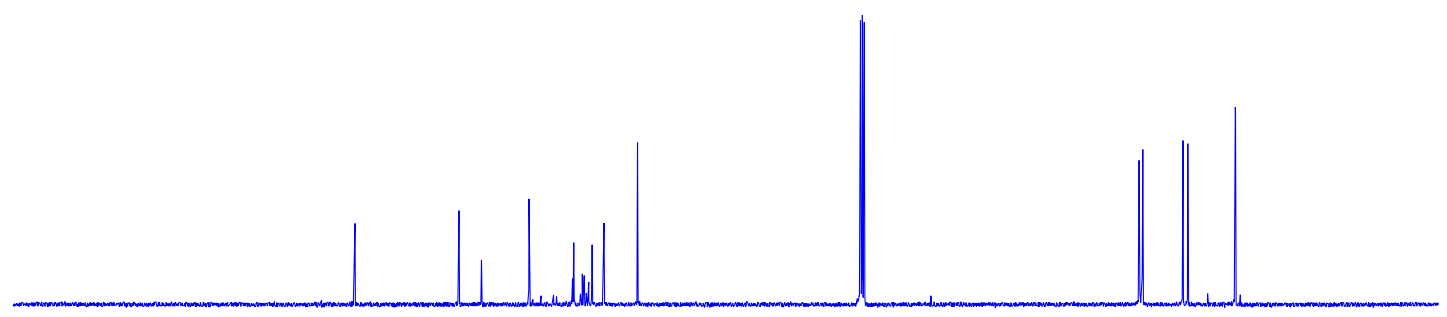

$\begin{array}{lllllllllllllllllllllllllllllllllll}210 & 200 & 190 & 180 & 170 & 160 & 150 & 140 & 130 & 120 & 110 & 100 & 50 & 80 & 70 & 60 & 50 & 40 & 30 & 20 & 10 & 0 & -10\end{array}$ 


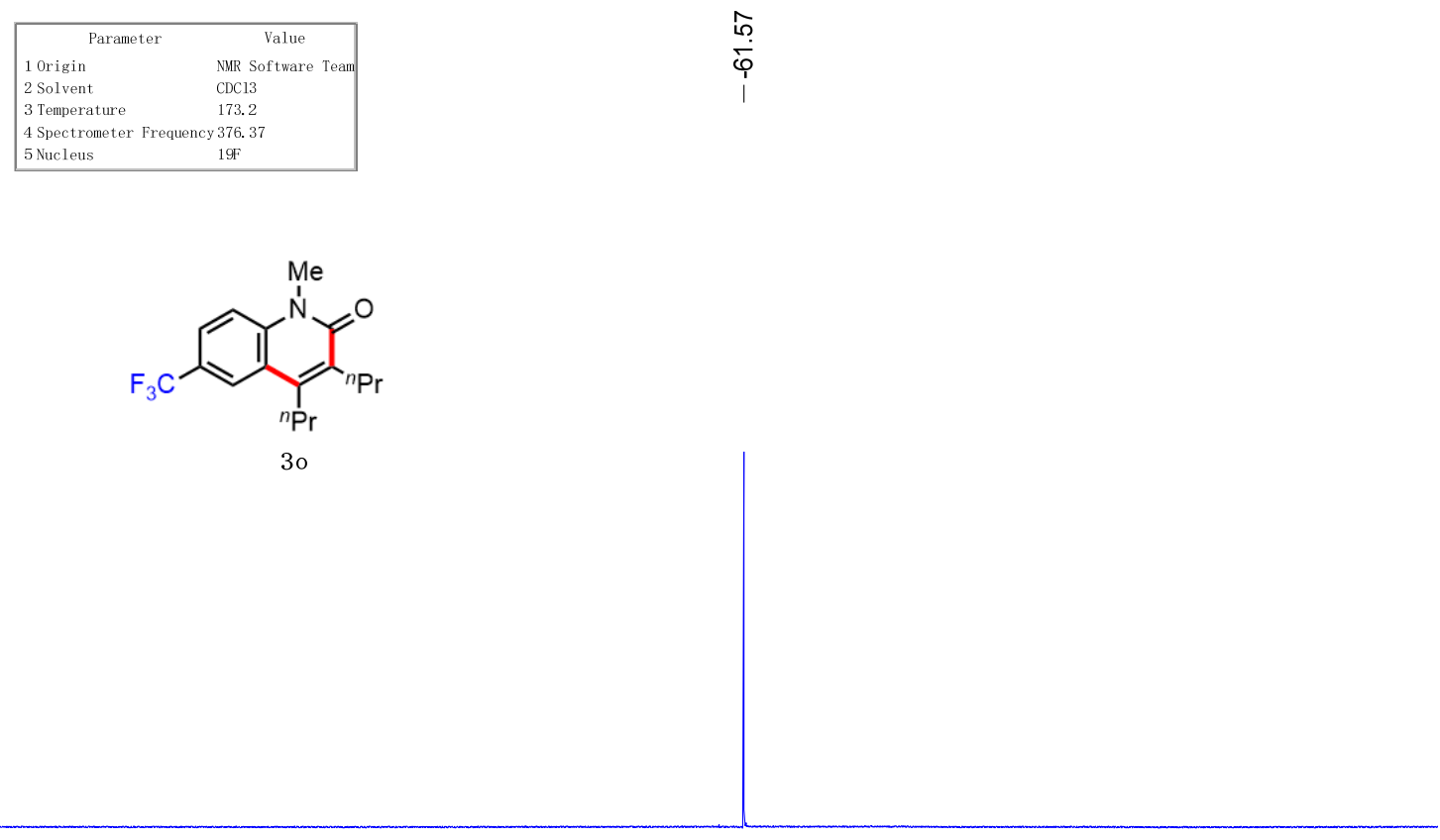

$\begin{array}{lllllllllllllllllllllllll}200 & 180 & 160 & 140 & 120 & 100 & 80 & 60 & 40 & 20 & 0 & -20 & -40 & -60 & -80 & -100 & & -140 & -160 & -180 & -200 & -240 & -280\end{array}$

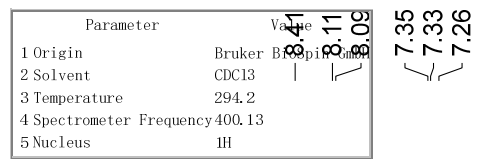

할

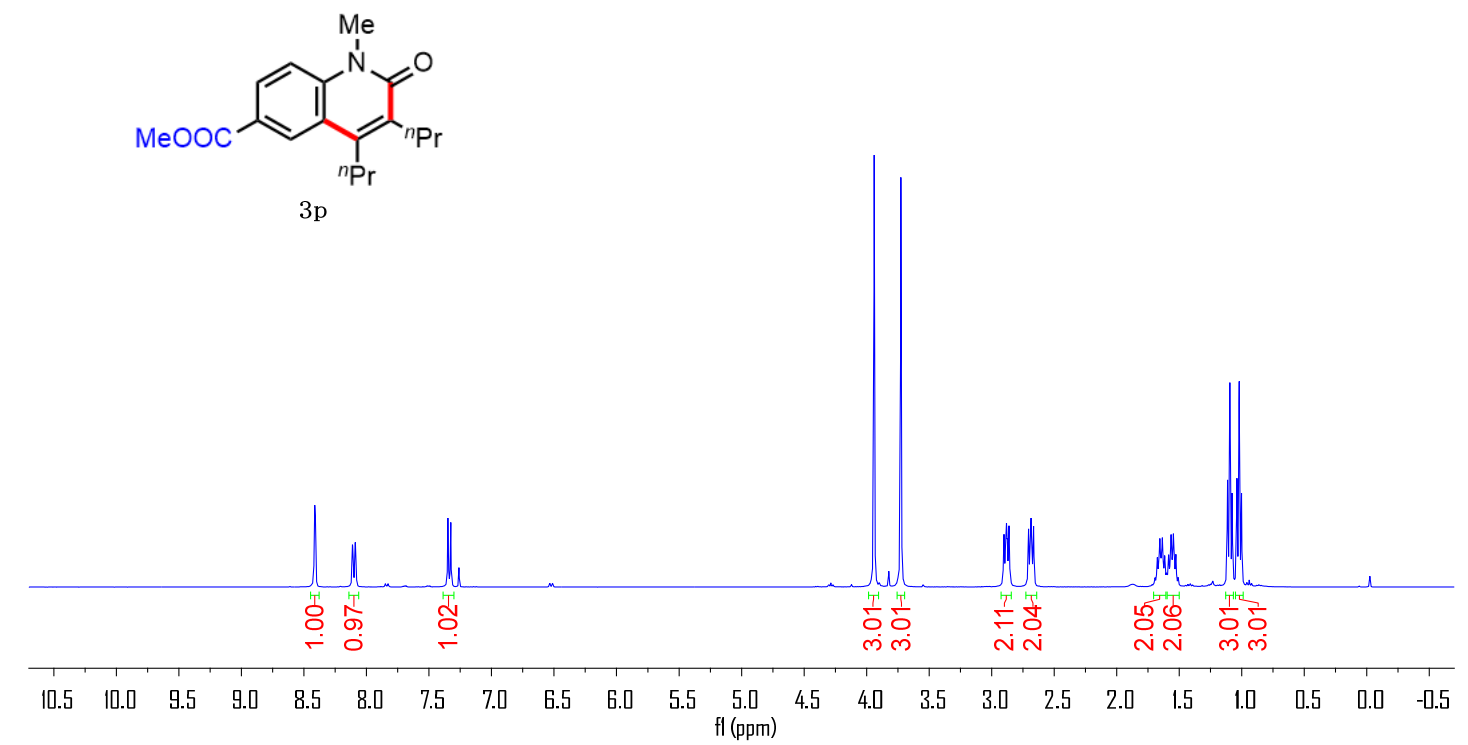




\begin{tabular}{|c|c|}
\hline \multicolumn{2}{|r|}{ Valup एீ } \\
\hline 1 Origin & Bruker Biosegi 영 \\
\hline 2 Solvent & $\mathrm{CDCl}_{2} \stackrel{0}{\leftarrow}$ \\
\hline 3 Temperature & 296.0 \\
\hline \multicolumn{2}{|c|}{4 Spectrometer Frequency 100.61} \\
\hline 5 Nucleus & $13 \mathrm{C}$ \\
\hline
\end{tabular}

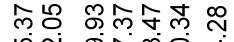

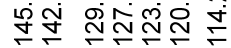

i 鸟店

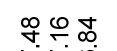

กN゚
กิ

กิ

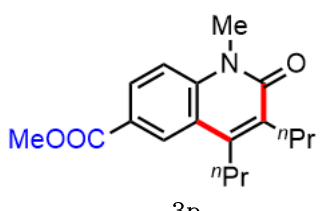

$3 p$

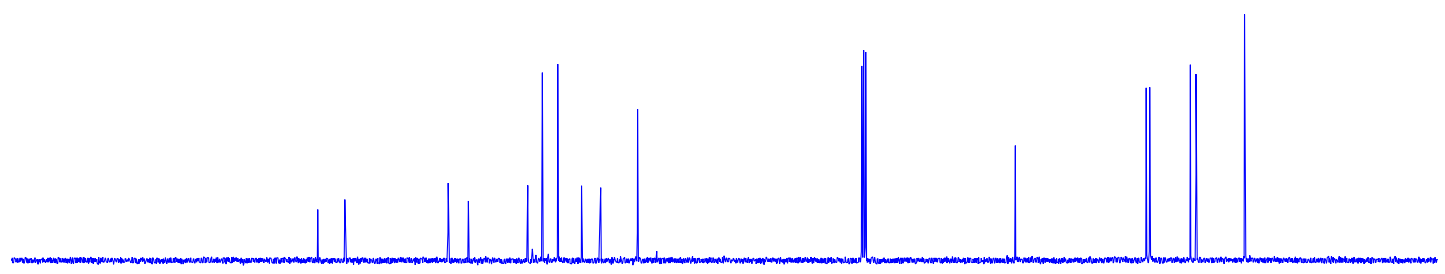

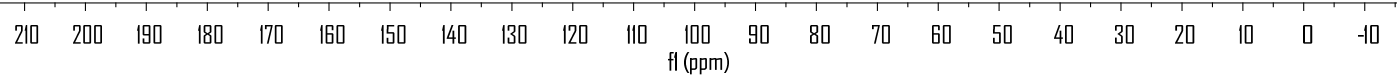

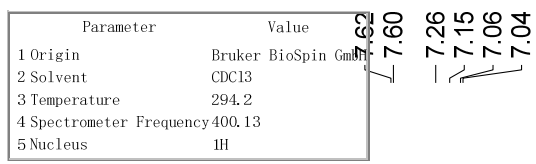

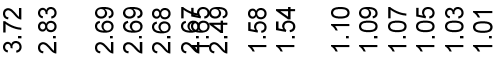

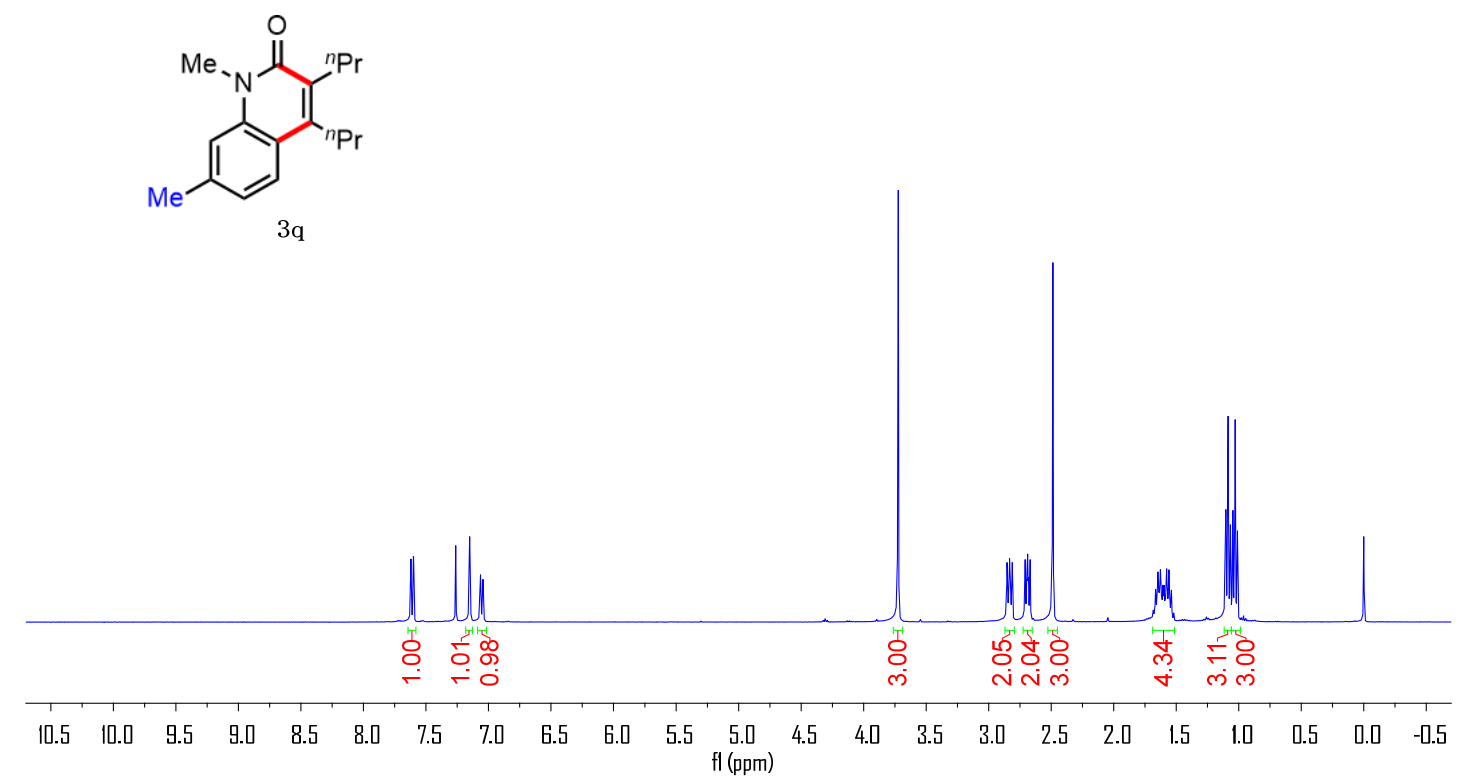

$3 q$
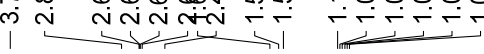

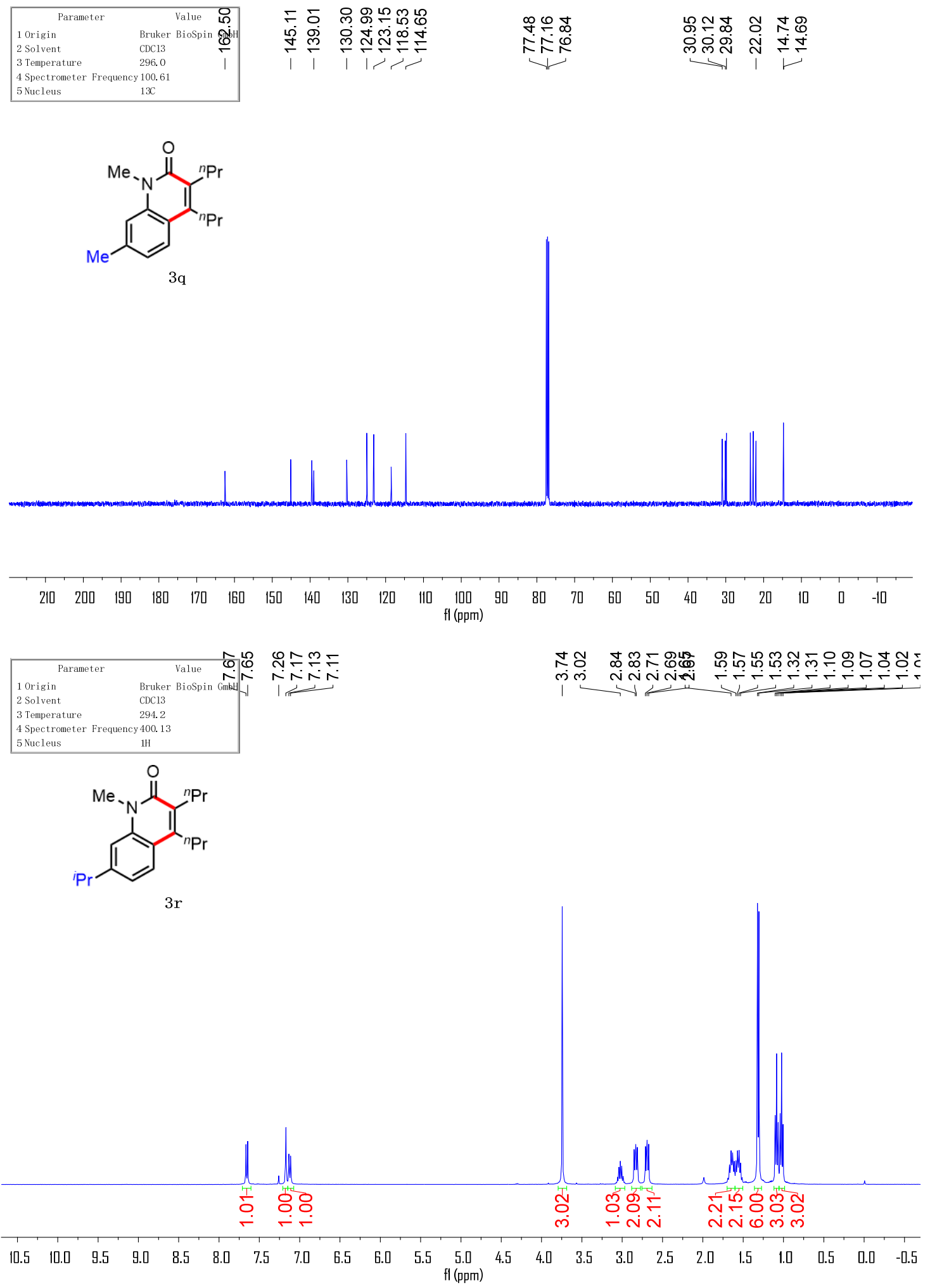

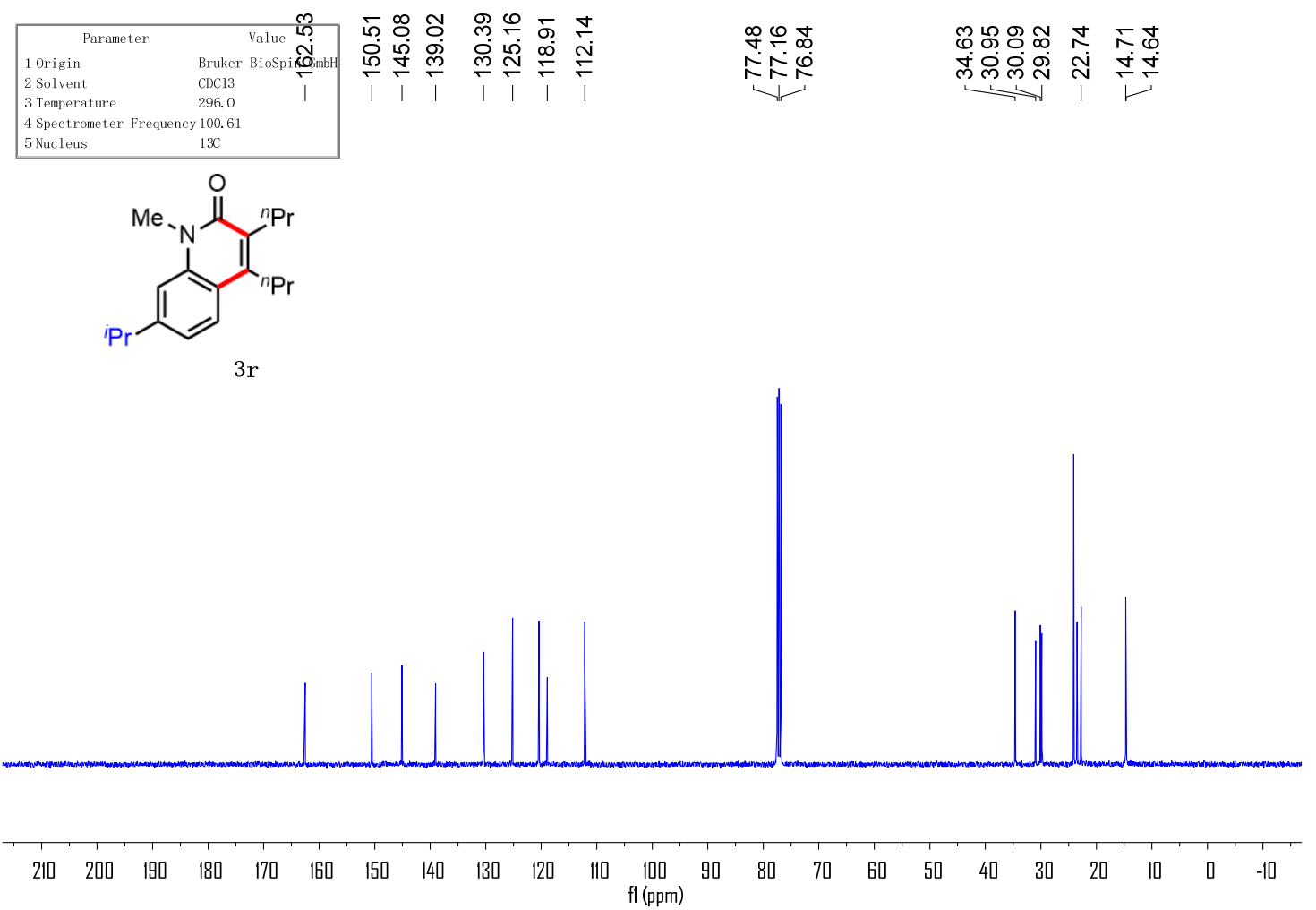

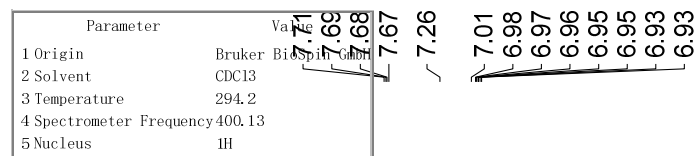

\%<smiles>CCCc1c(C(C)C)c(=O)n(C)c2cc(F)ccc12</smiles>

$3 \mathrm{~s}$

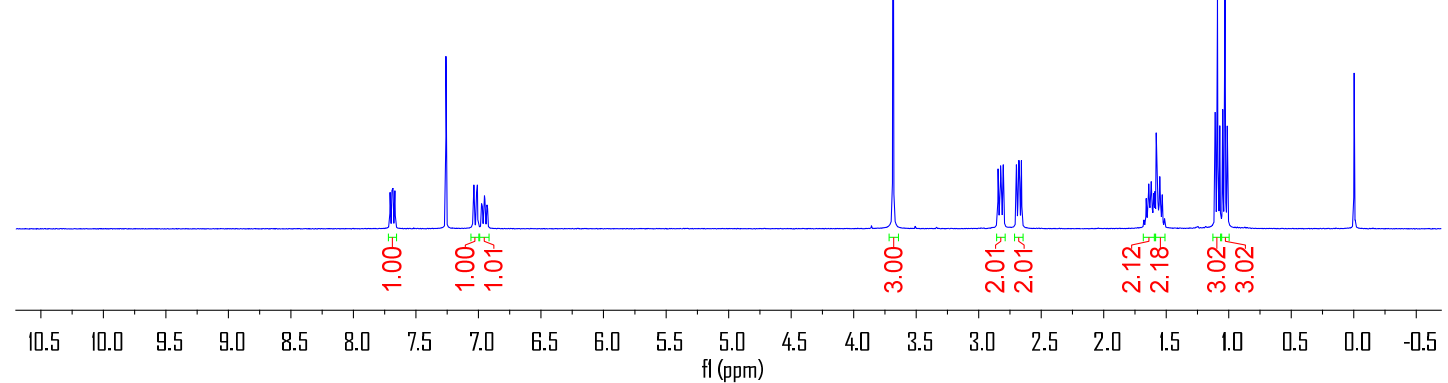




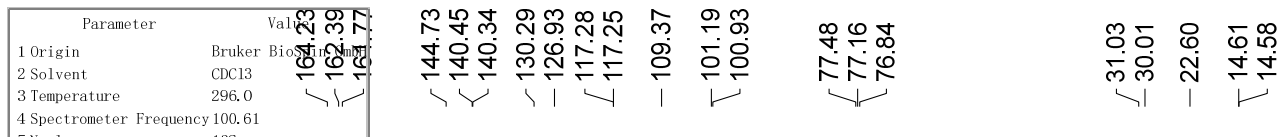

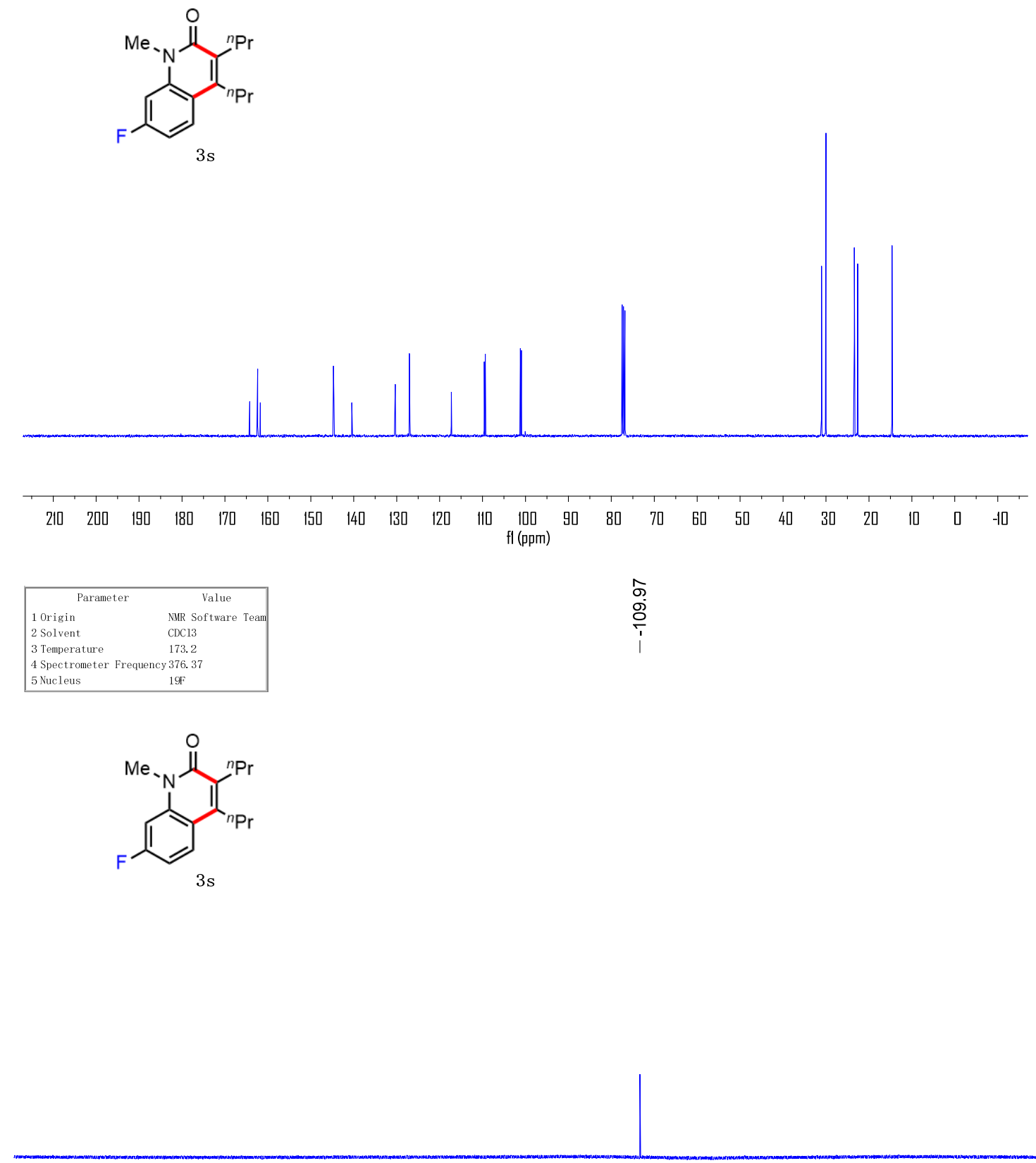

$3 \mathrm{~s}$

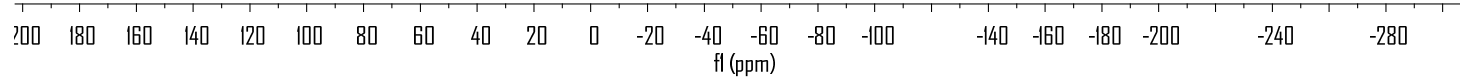




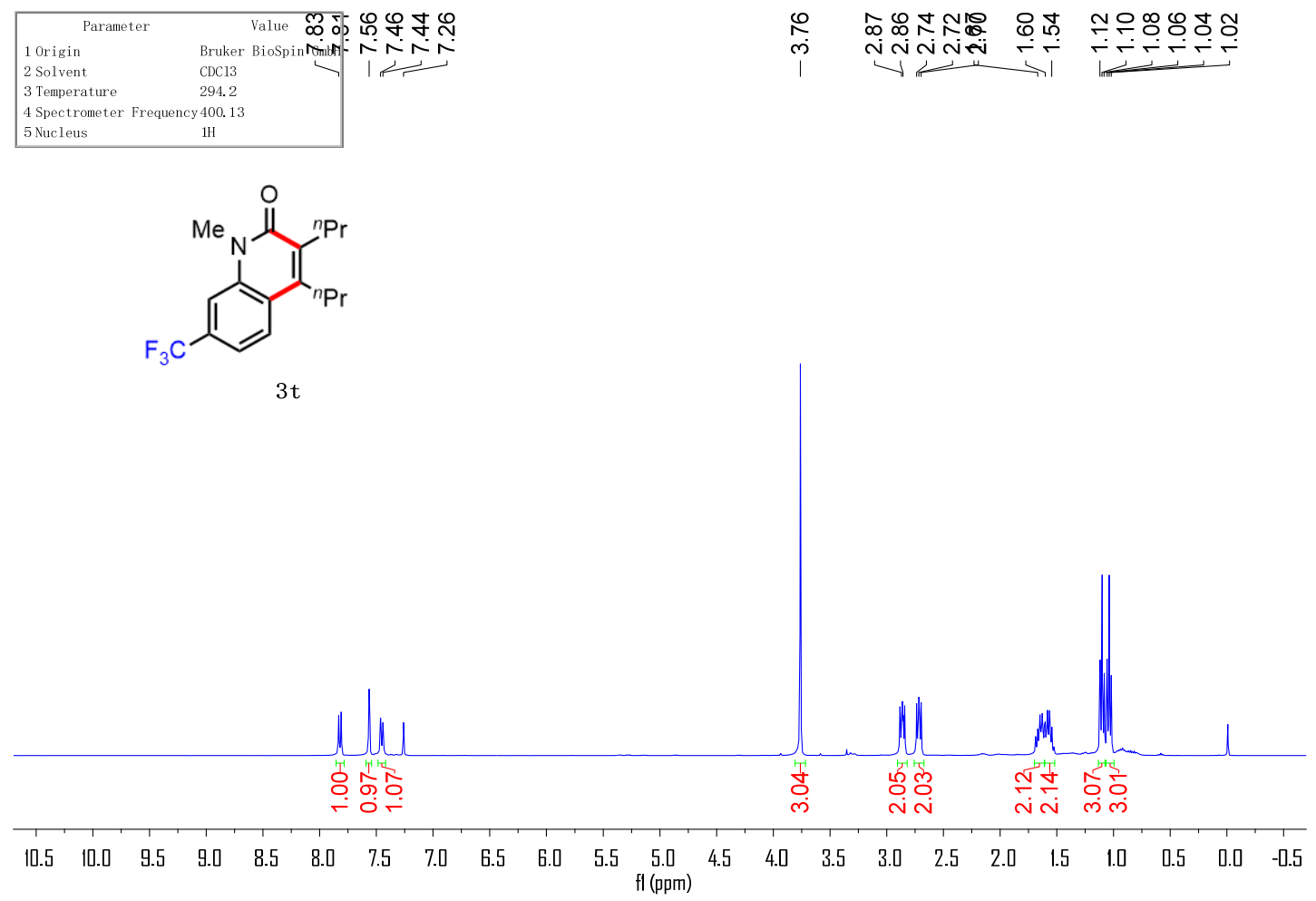

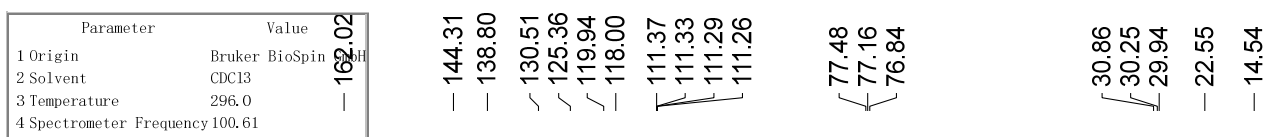

4 Spectrometer Frequency 100.6
5 Nucleus
$13 \mathrm{C}$

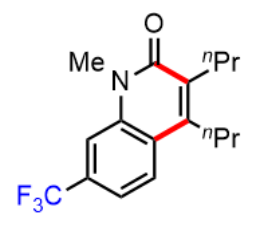

$3 \mathrm{t}$

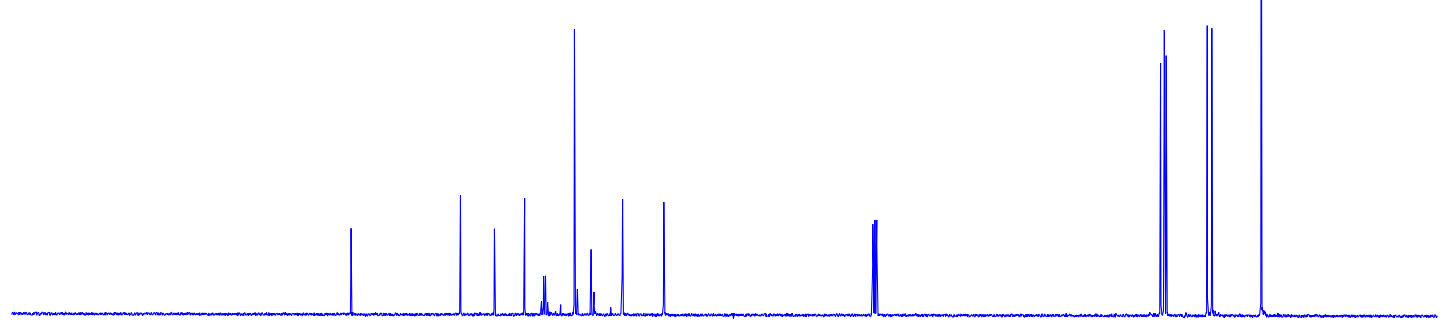

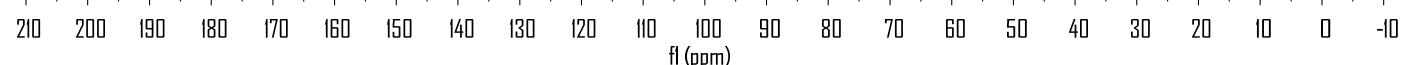




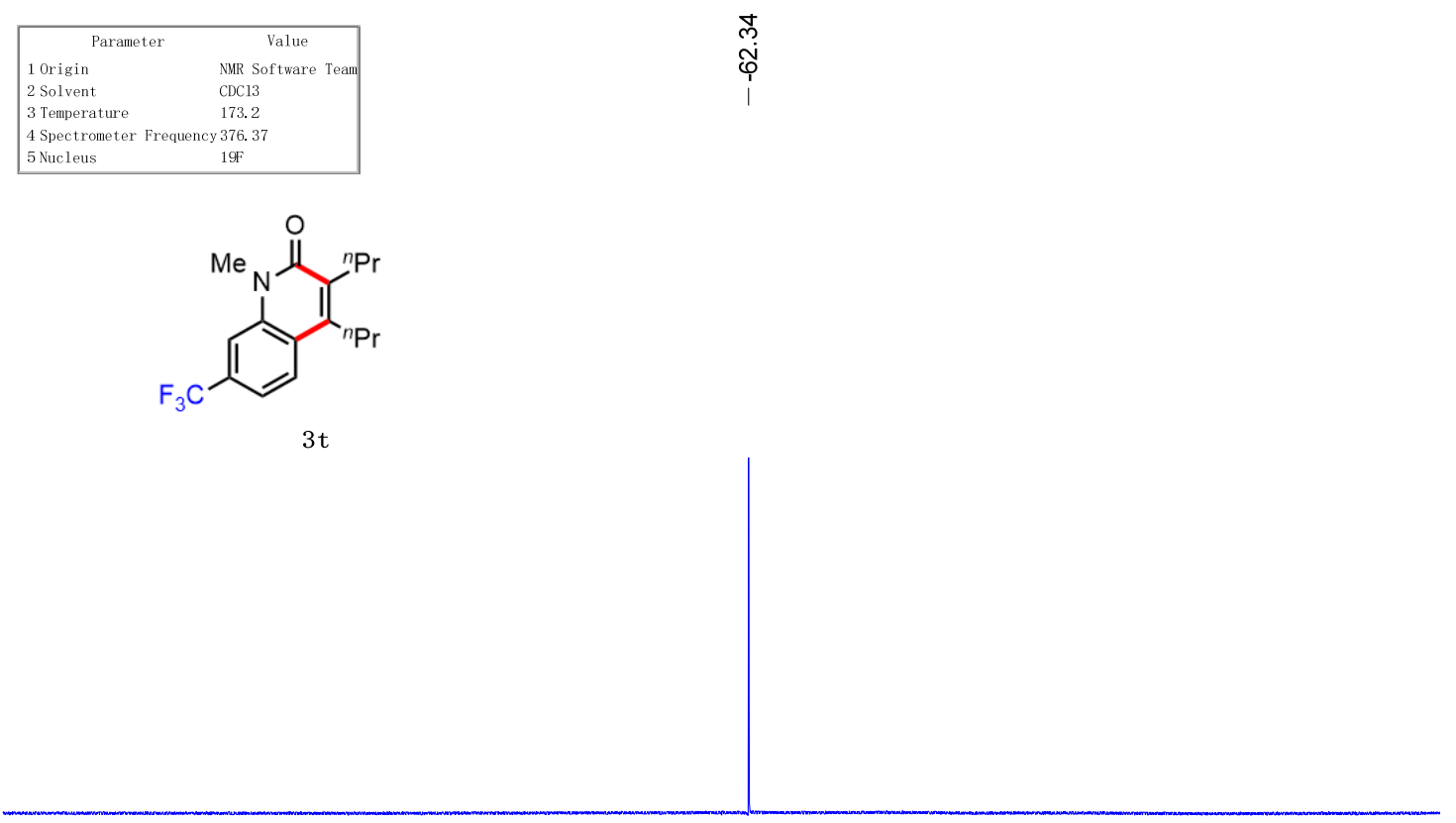

$\begin{array}{lllllllllllllllllllllllllll}200 & 180 & 160 & 140 & 120 & 100 & 80 & 60 & 40 & 20 & 0 & -20 & -40 & -60 & -80 & -100 & & -140 & -160 & -180 & -200 & -240 & -280\end{array}$

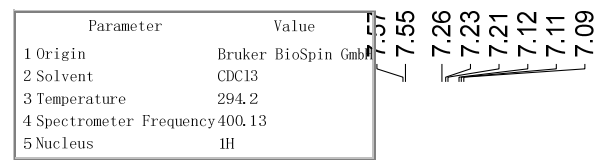

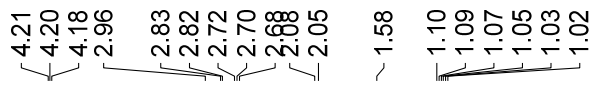

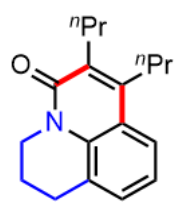

$3 u$

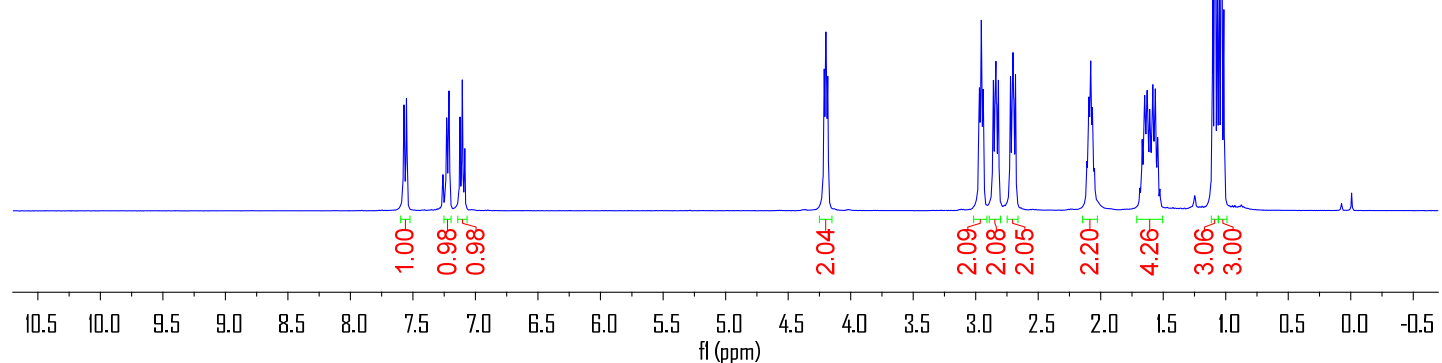



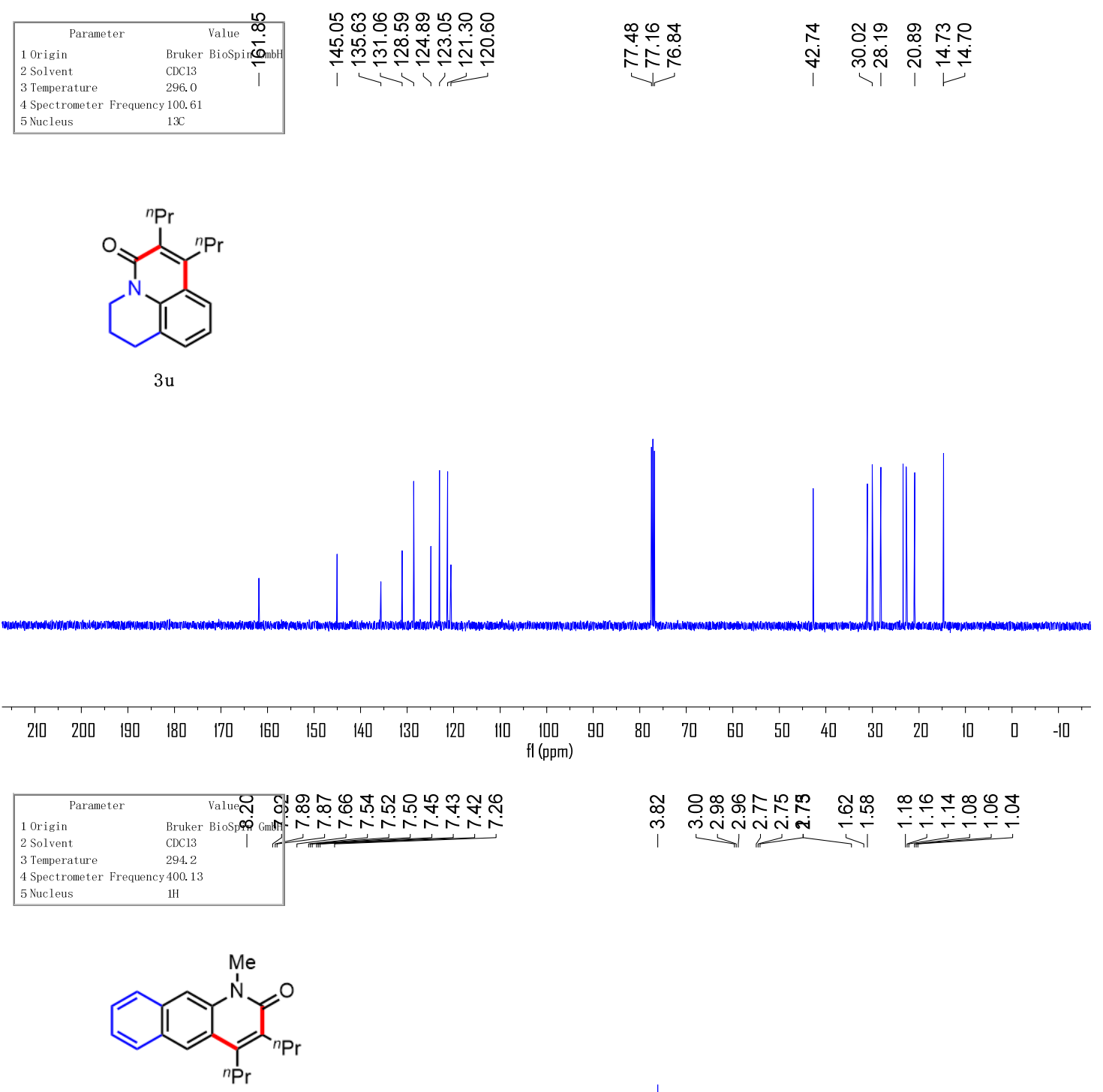

$3 v$

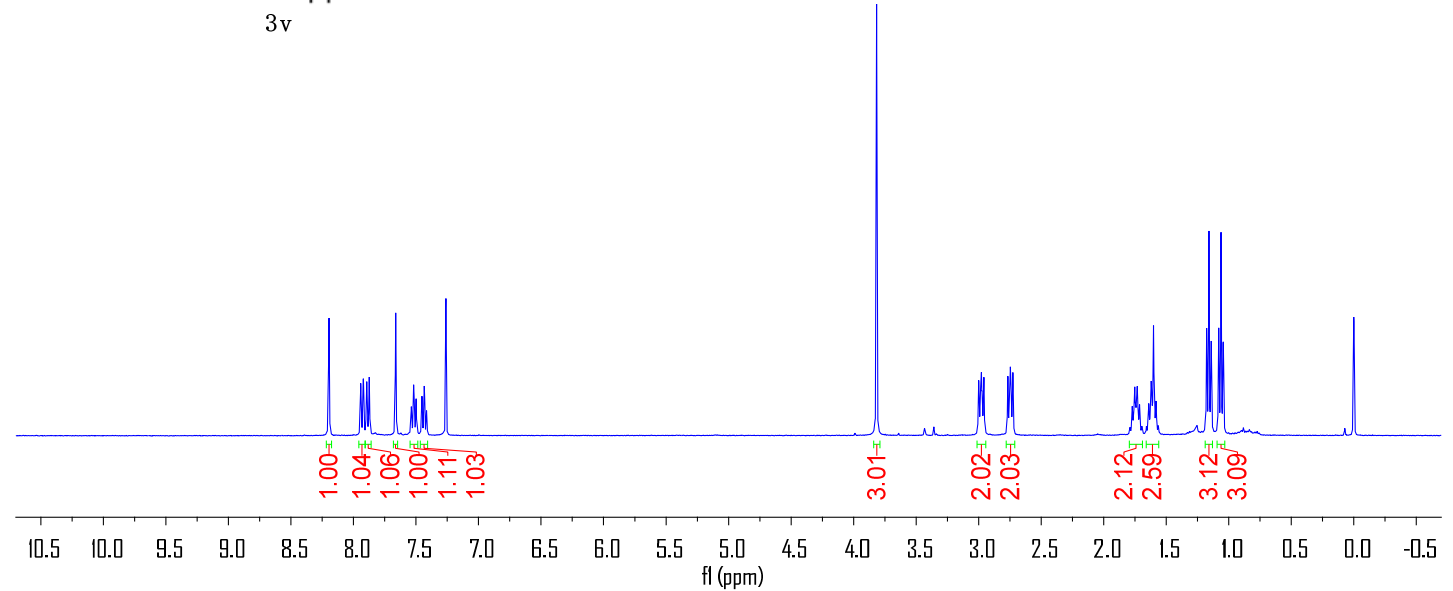



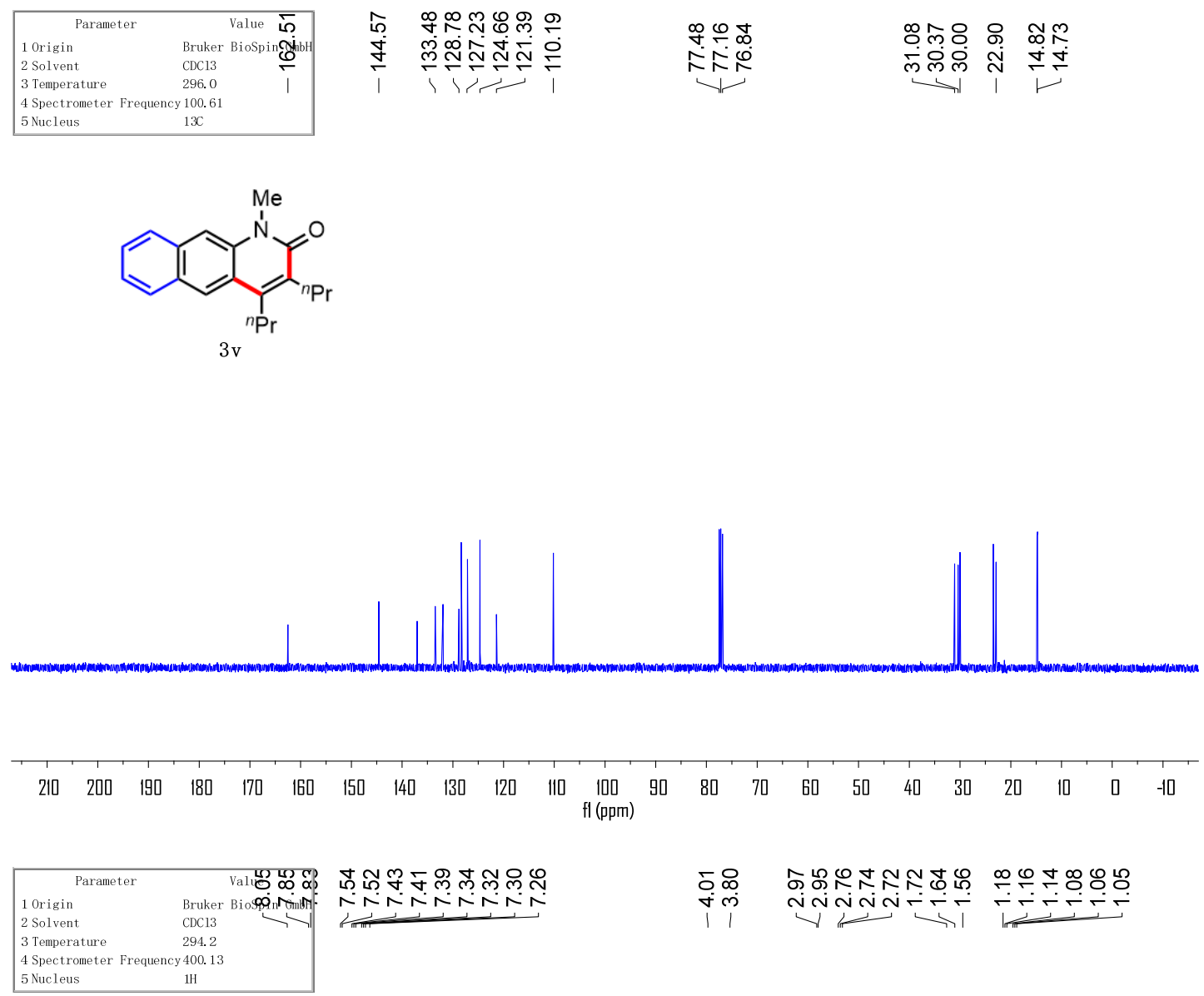

il

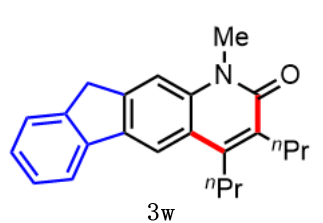

$3 w$

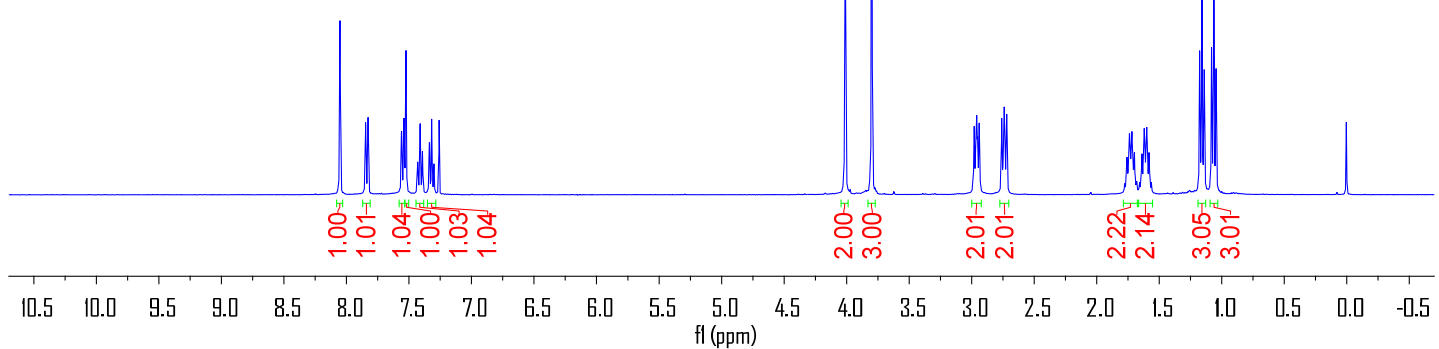




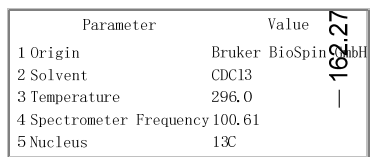

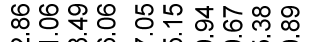

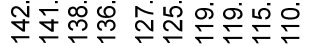
i।

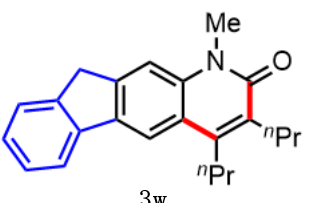

$3 w$

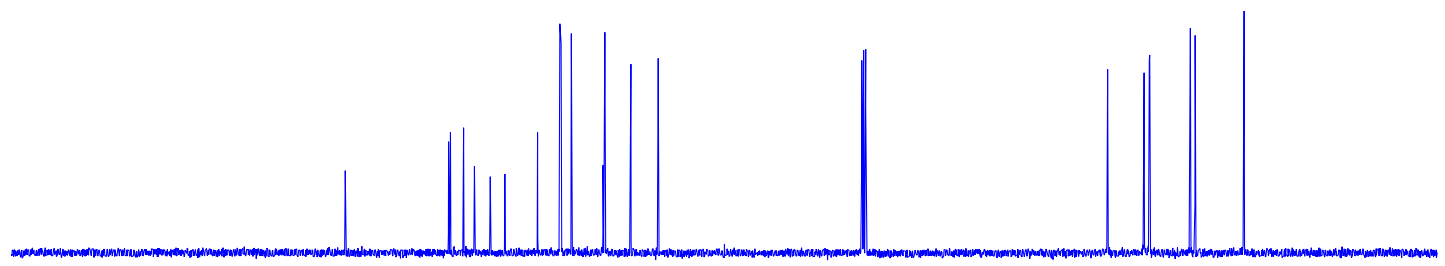

$\begin{array}{llllllllllllllllllllllll}210 & 200 & 190 & 180 & 170 & 160 & 150 & 140 & 130 & 120 & 110 & 100 & 90 & 80 & 70 & 60 & 50 & 40 & 30 & 20 & 10 & 0 & -10\end{array}$

\begin{tabular}{|ll}
\hline \multicolumn{1}{|c|}{ Parameter } \\
1 Origin \\
2 Solvent & Balue \\
3 Temperature & CDC13 Bospin GmbH \\
4 Spectrometer Frequency 400.13 \\
5 Nucleus & 1H
\end{tabular}

i<smiles>CCCc1c(C(C)C)c(=O)n(C)c2ccsc12</smiles>

$3 \mathrm{x}$

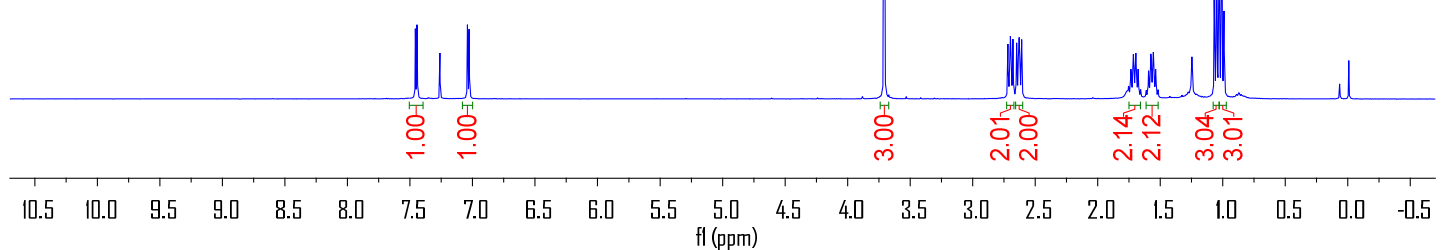




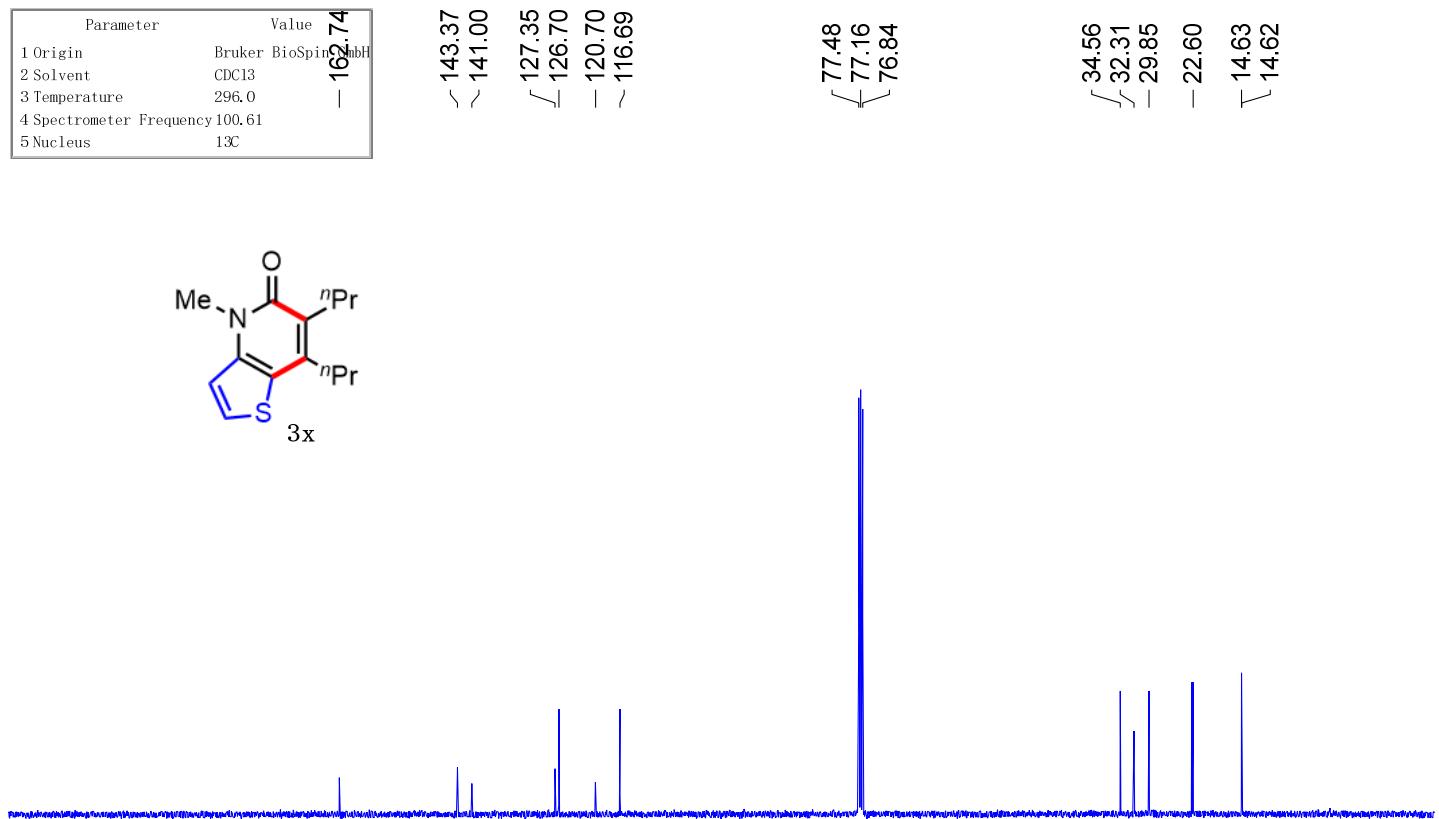

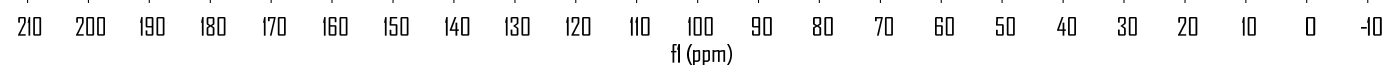

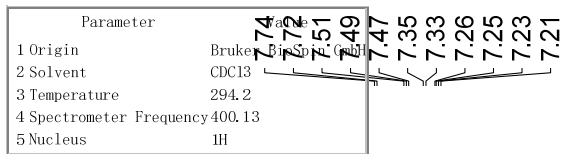

草<smiles>Cn1c(=O)c(C(C)(C)C)c(C(C)(C)C)c2ccccc21</smiles>

$4 a$

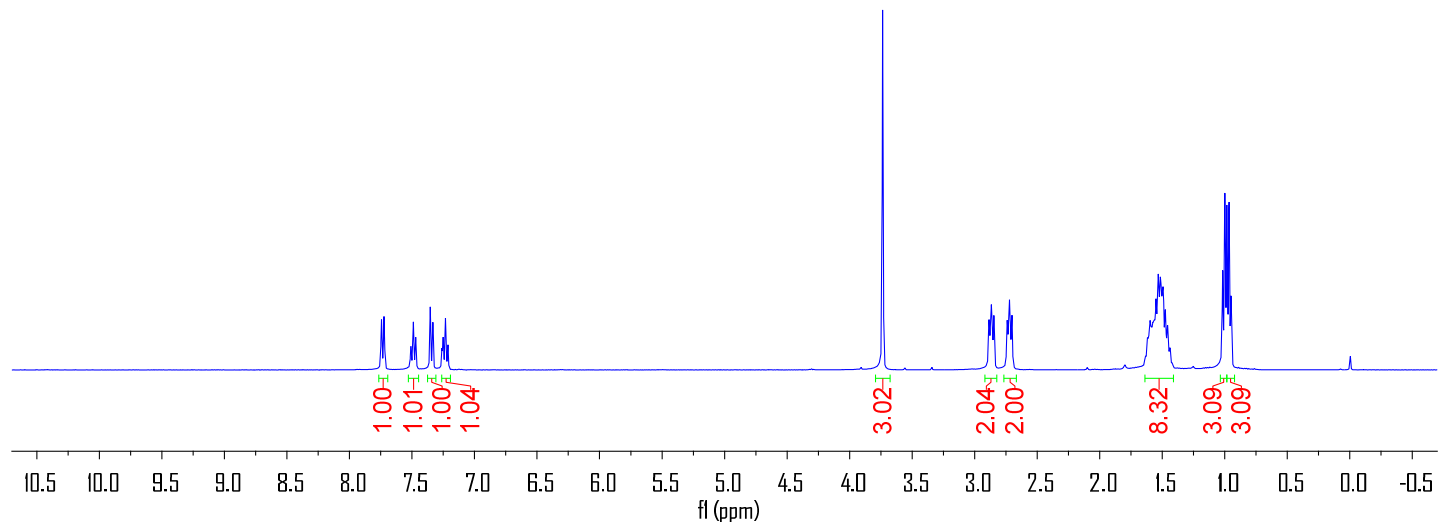




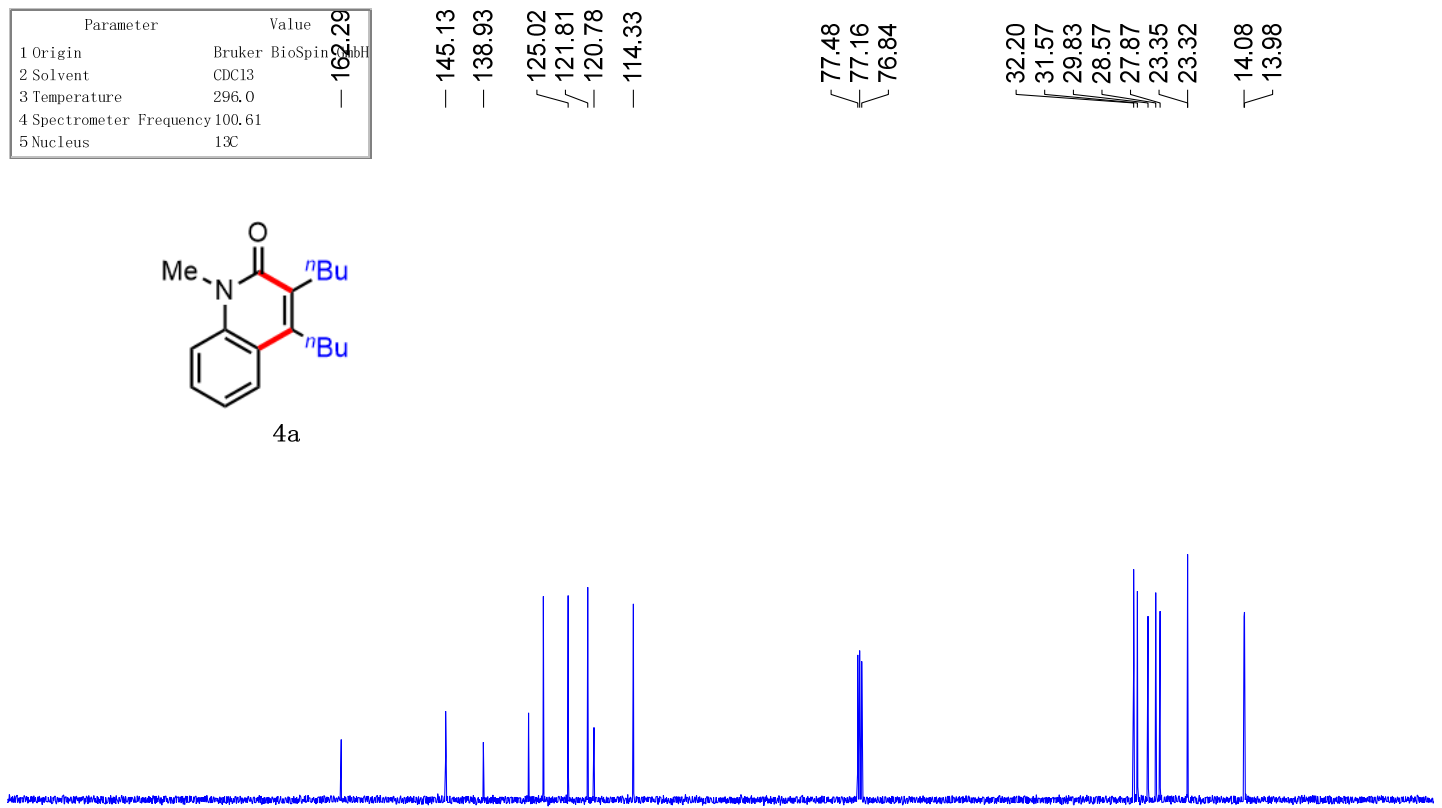

$\begin{array}{lllllllllllllllllllllllll}210 & 200 & 190 & 180 & 170 & 160 & 150 & 140 & 130 & 120 & 110 & 100 & 90 & 80 & 70 & 60 & 50 & 40 & 30 & 20 & 10 & 0 & -10\end{array}$

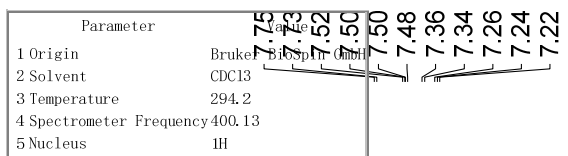

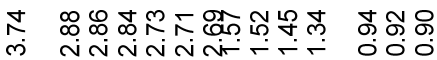<smiles>Cn1c(=O)c(C=[W])c(C=[W])c2ccccc21</smiles>

$4 \mathrm{~b}$

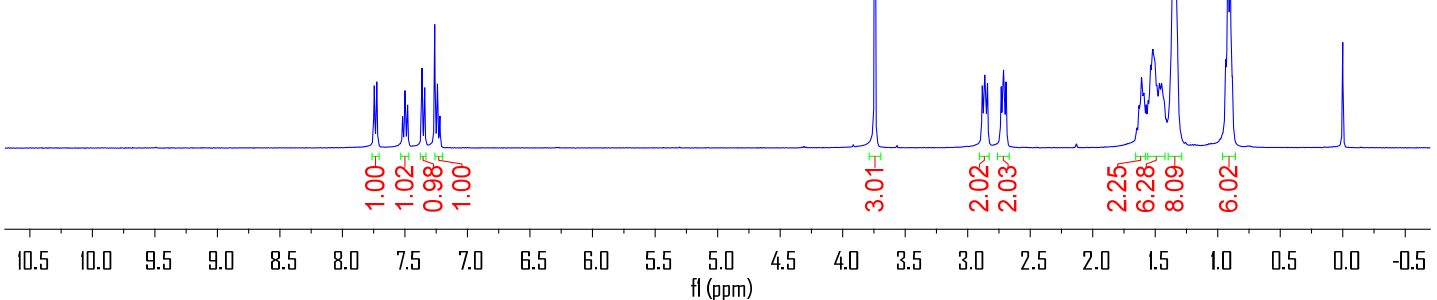




\begin{tabular}{|ll|}
\hline \multicolumn{1}{|c}{ Parameter } & Value \\
1 Origin & Bruker BioSpin \\
2 Solvent & CDC13 \\
3 Temperature & 296.0 \\
4 Spectrometer & Frequency 100.61 \\
5 Nucleus & $13 \mathrm{C}$ \\
\hline
\end{tabular}

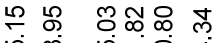

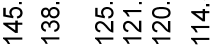

1
迎亲

소

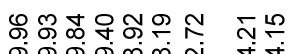

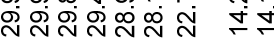<smiles>Cn1c(=O)c(C=[W])c(C=[W])c2ccccc21</smiles>

$4 \mathrm{~b}$

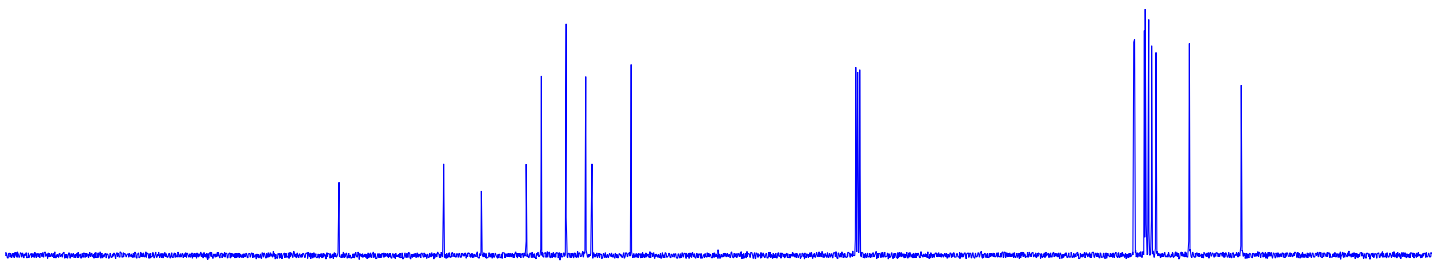

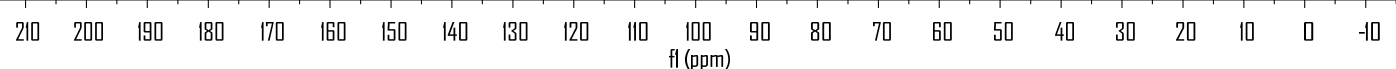

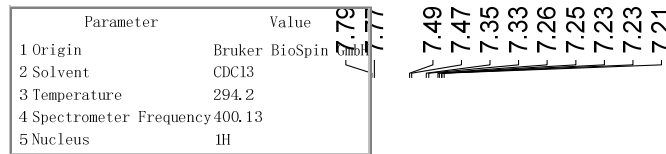

음 ๘

m n n v v v
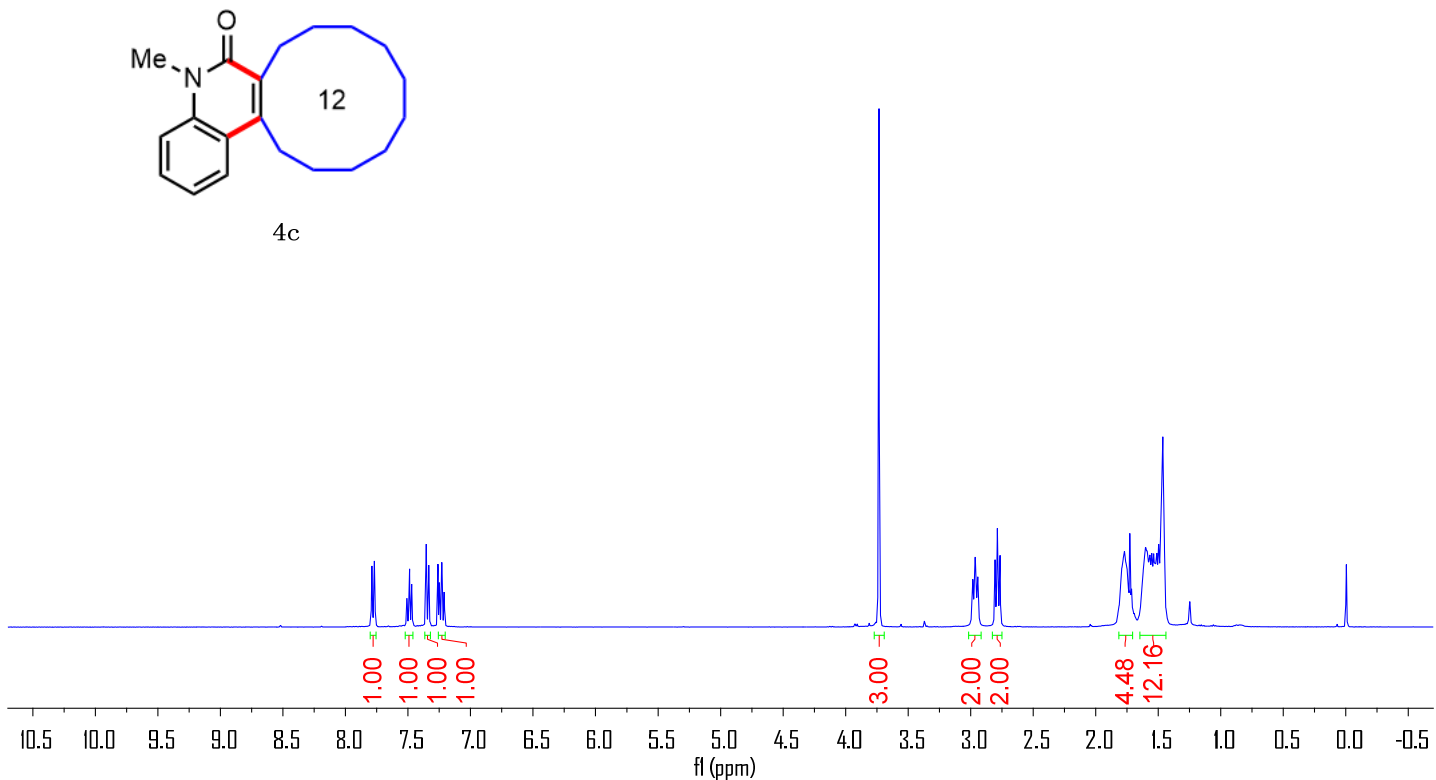


\begin{tabular}{|ll|}
\hline \multicolumn{1}{|c|}{ Parameter } & Value \\
1 Origin & Bruker BioSpin \\
2 Solvent & CDC13 \\
3 Temperature & 296.0 \\
4 Spectrometer Frequency 100.61 \\
5 Nucleus & $13 \mathrm{C}$ \\
\hline
\end{tabular}

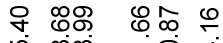

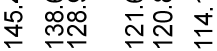

i $\stackrel{\infty}{\sim} \stackrel{\infty}{N}$

走是

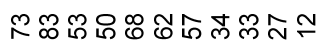

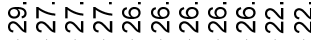<smiles>Cn1c(=O)c2c(c3ccccc31)CCCCCCCCCC2</smiles>

$4 \mathrm{c}$

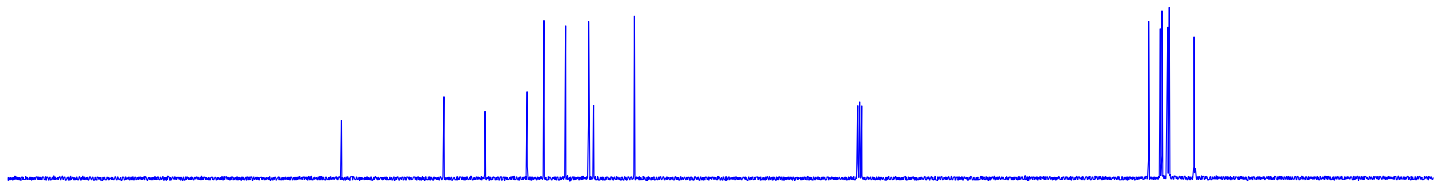

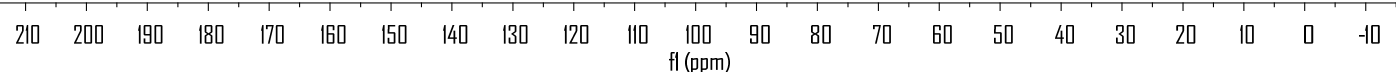

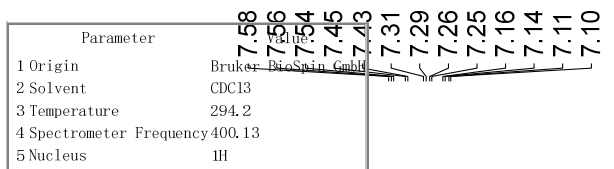<smiles>Cn1c(=O)c(-c2ccccc2)c(-c2ccccc2)c2ccccc21</smiles>

$4 \mathrm{~d}$

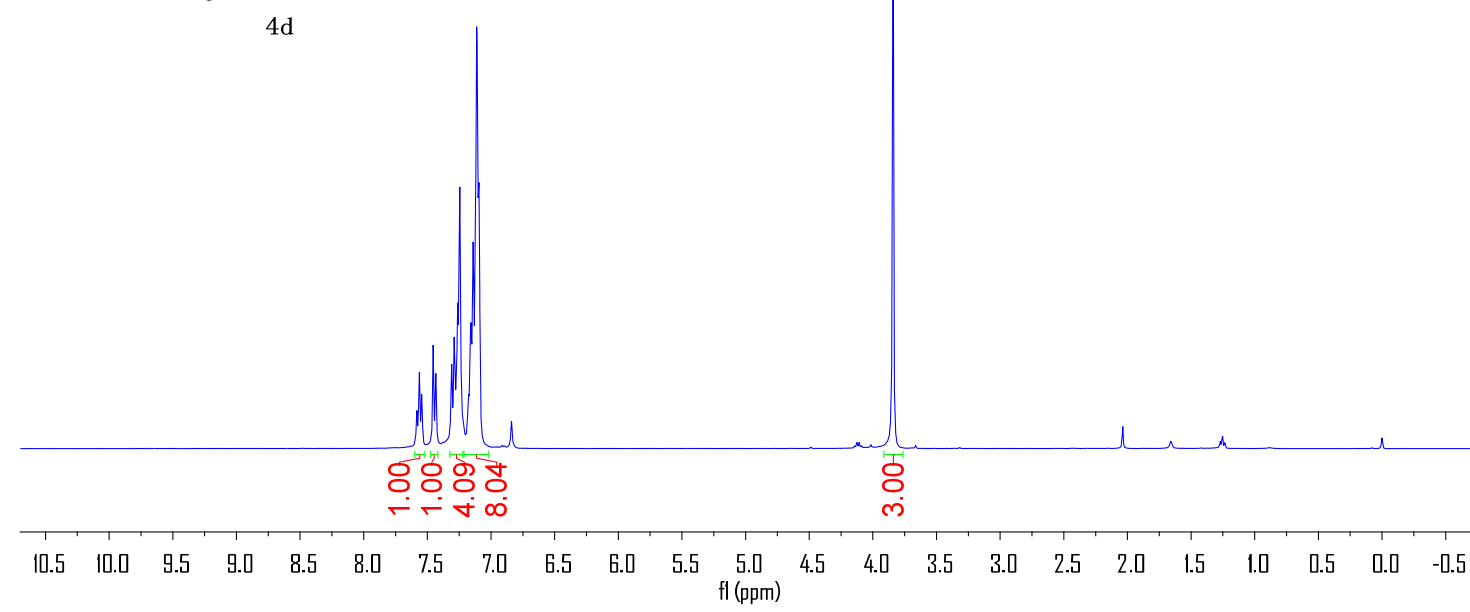



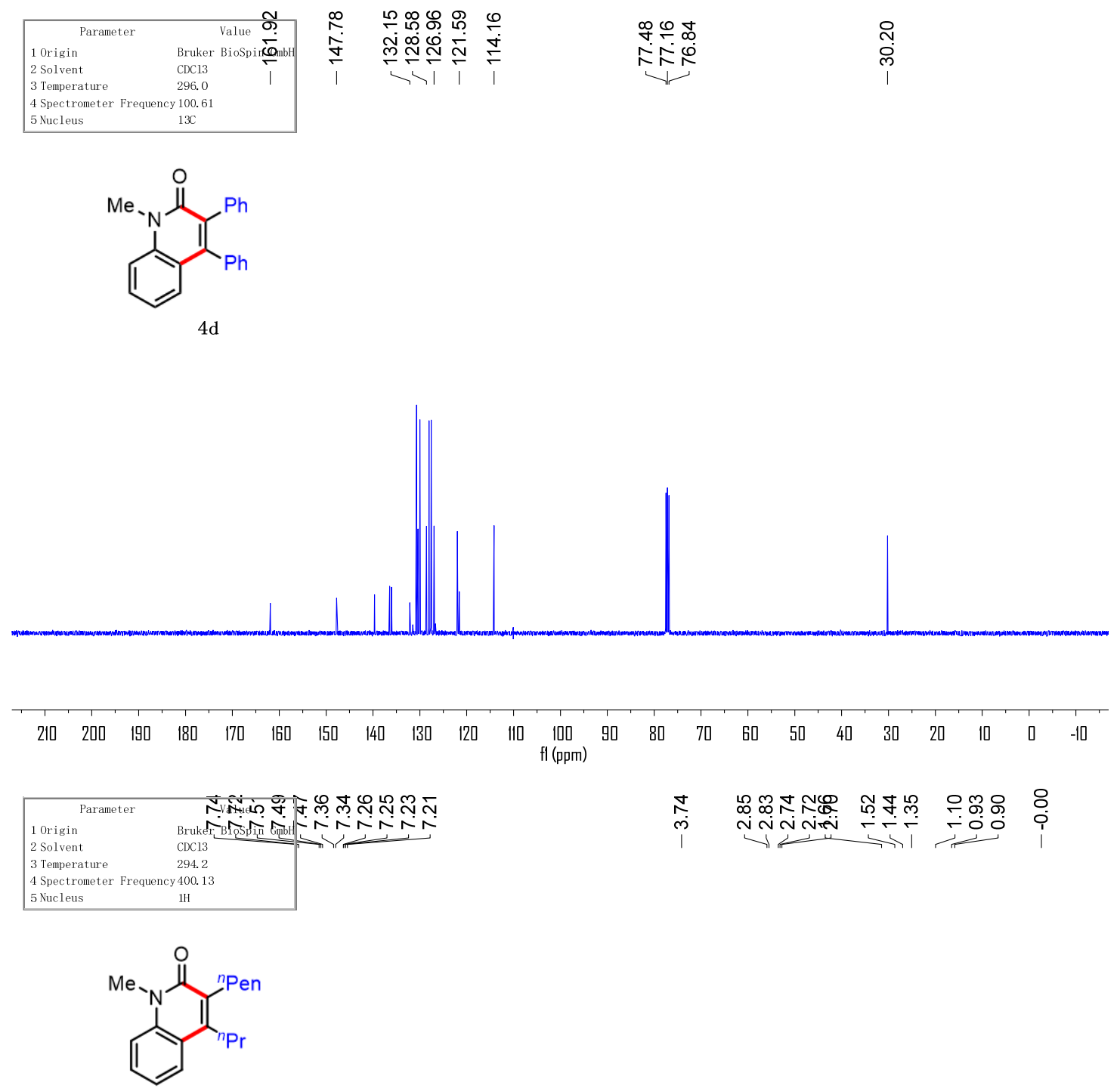

4 e

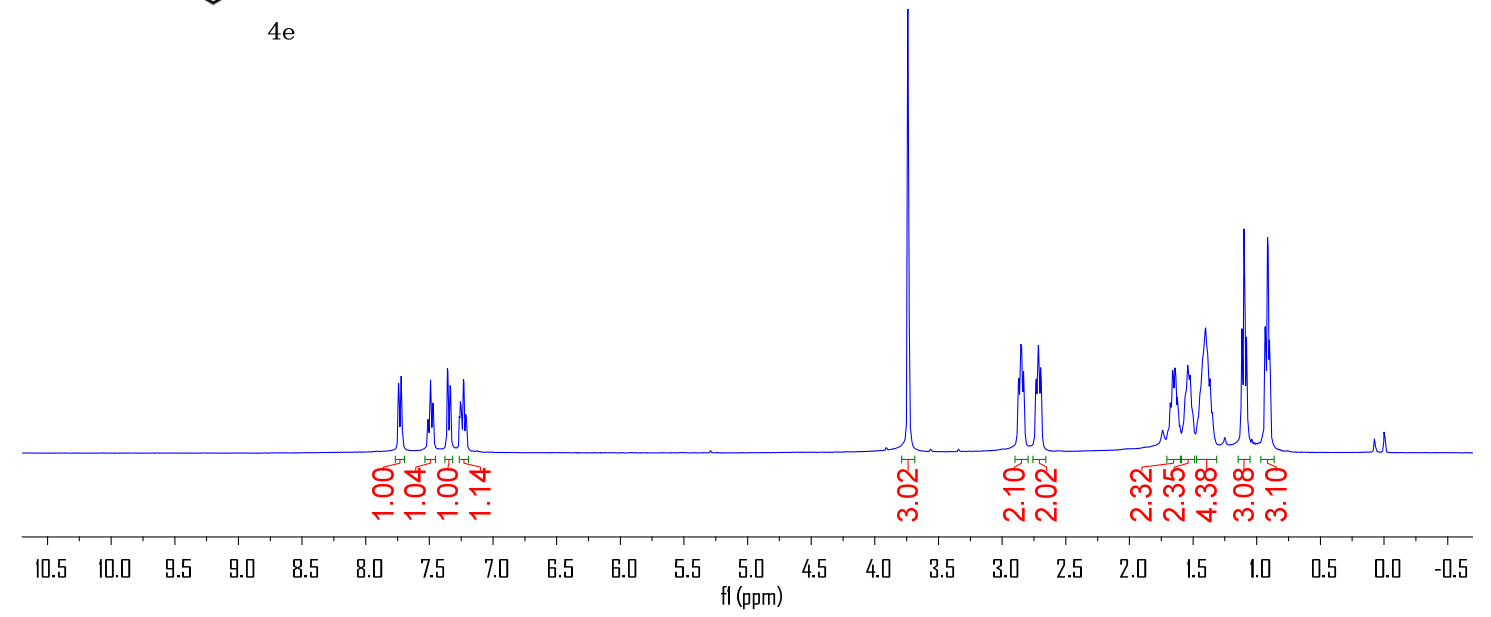



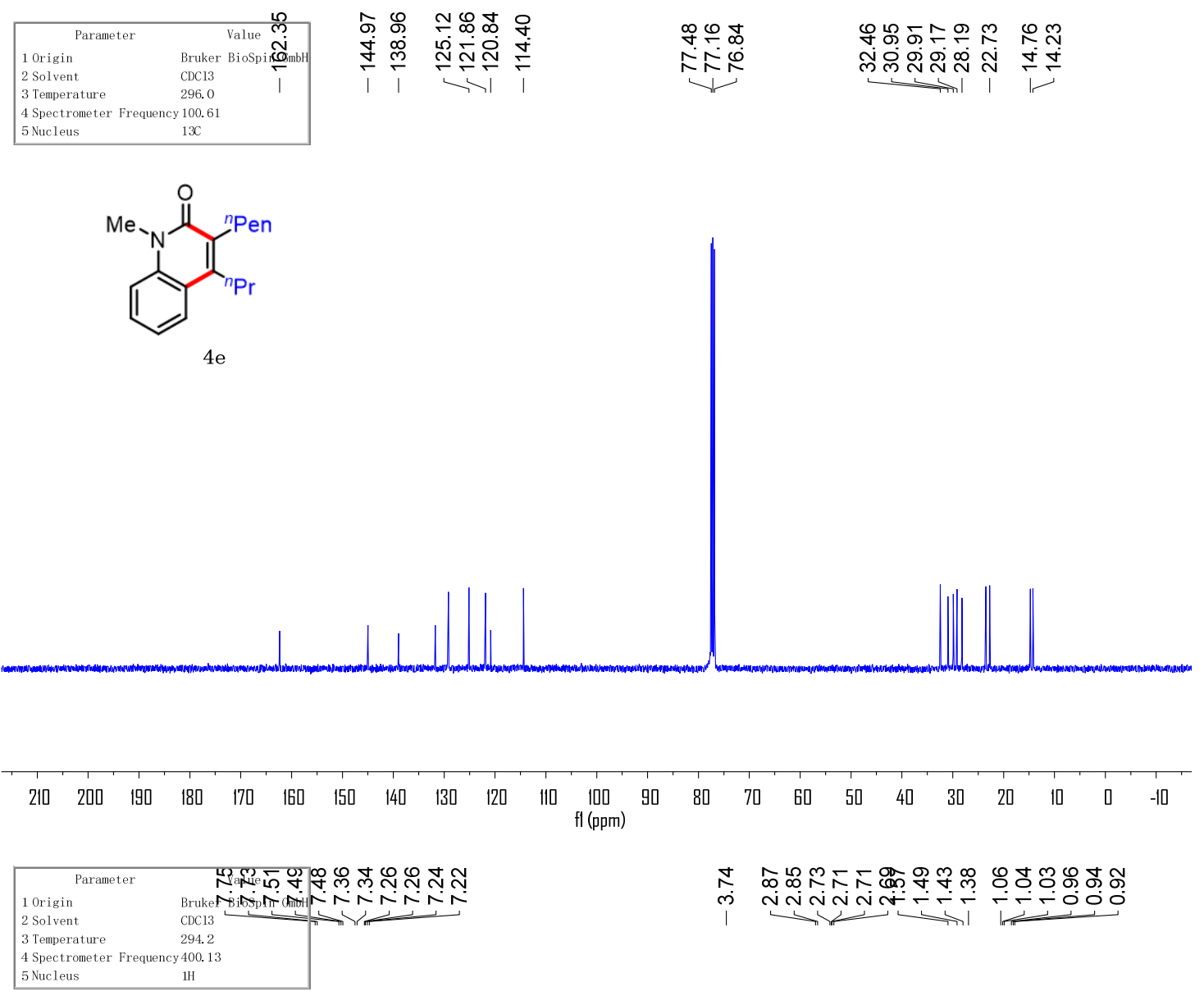

每<smiles>CCCc1c(C(=O)c2ccccc2)c2ccccc2n(C)c1=O</smiles>

$4 \mathrm{e}^{\prime}$

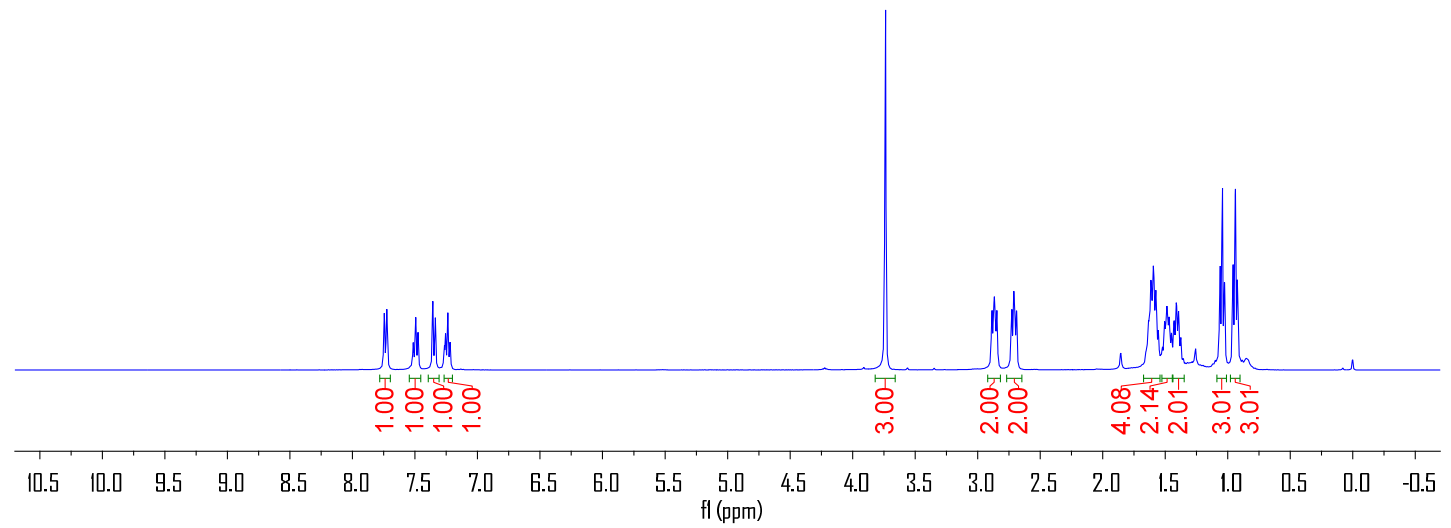




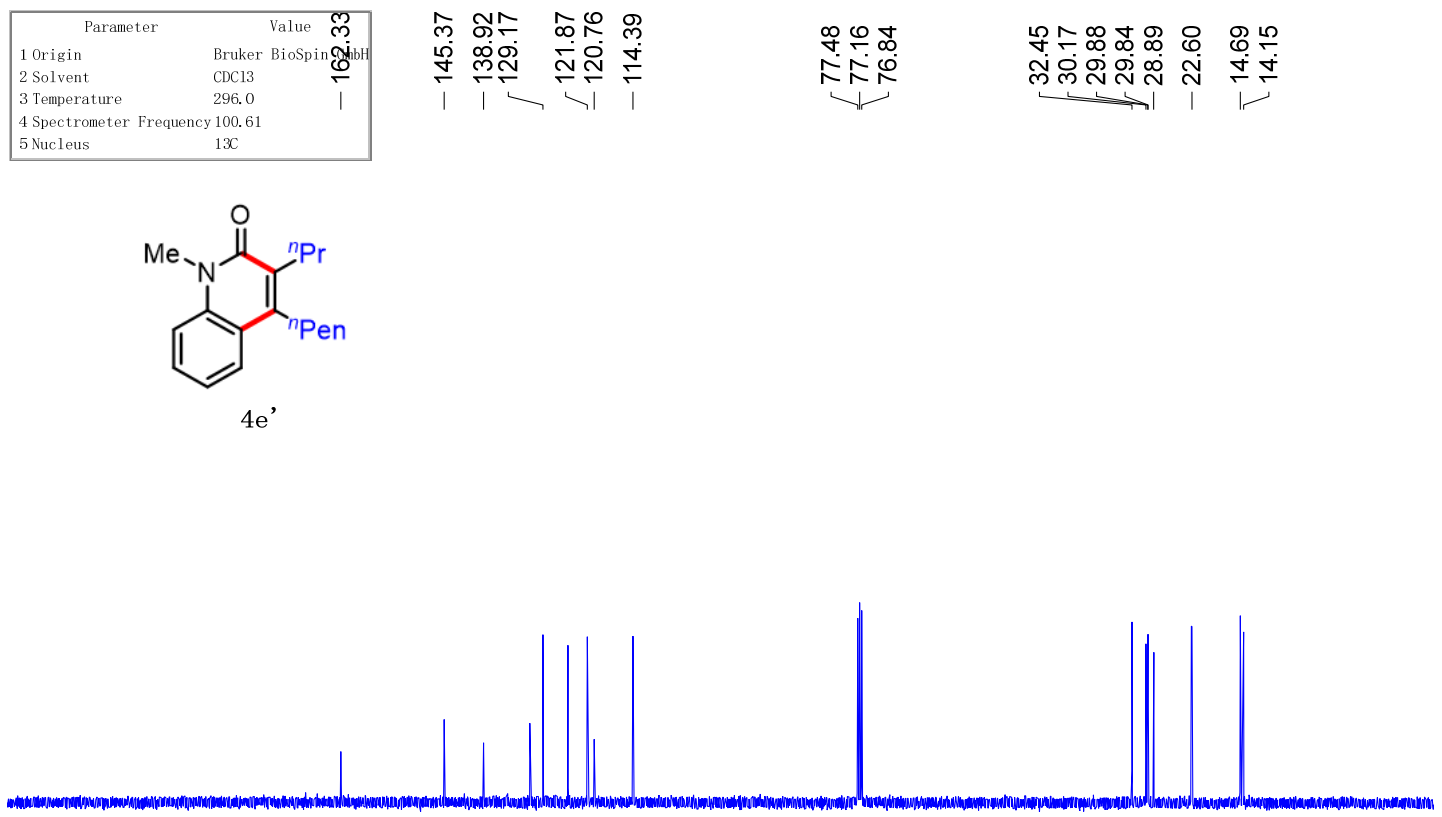

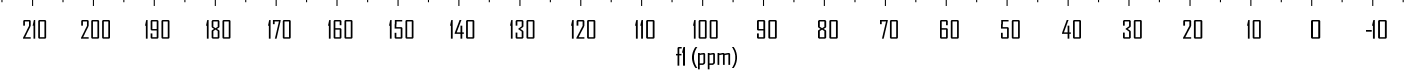

\begin{tabular}{|c|c|c|c|c|}
\hline Parameter & Value $\hat{~}$ & 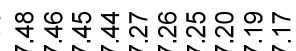 & $\hat{\emptyset}$ & $\overline{6}$ \\
\hline 1 Origin & Bruker BioSpin & HNTNNNTNNA & & i \\
\hline 2 Solvent & $\mathrm{CDC13}$ & & & \\
\hline $\begin{array}{l}3 \text { Temperature } \\
4 \text { Spectrometer Frequency. }\end{array}$ & $\begin{array}{l}294.2 \\
4400.13\end{array}$ & & & \\
\hline 5 Nucleus & $1 \mathrm{H}$ & & & \\
\hline
\end{tabular}<smiles>CCC(C)c1c(C)c2ccccc2n(C)c1=O</smiles>

$4 \mathrm{f}$

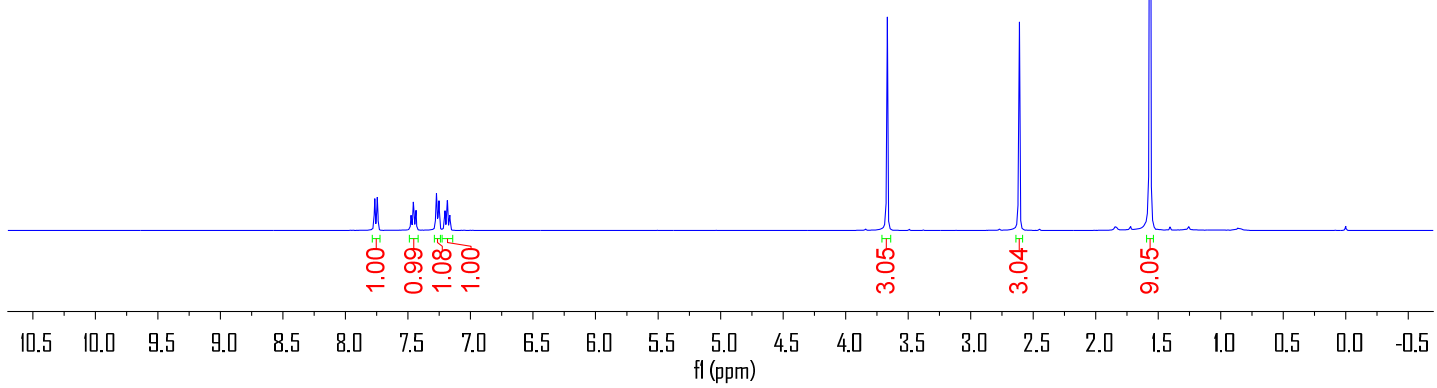




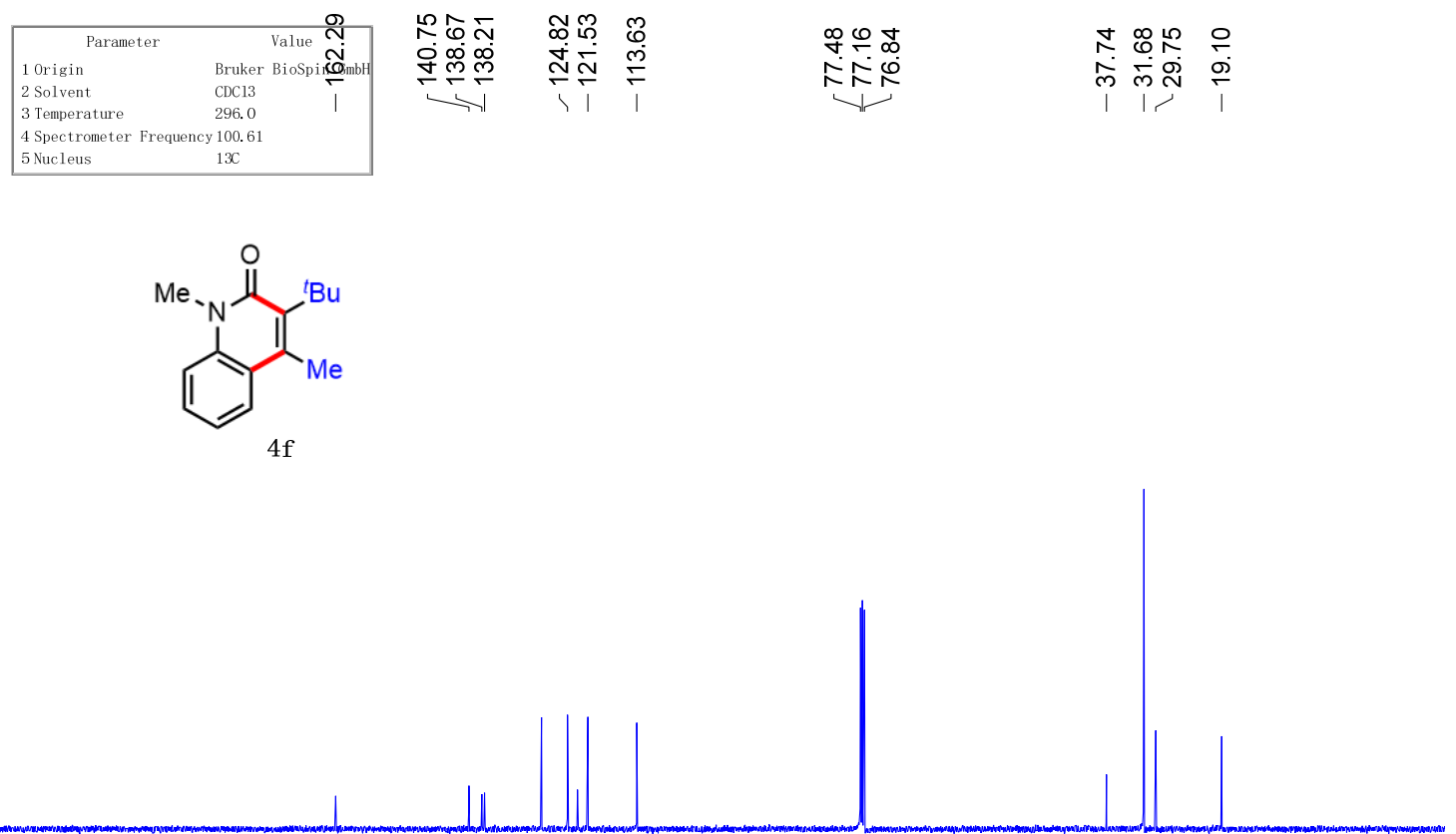

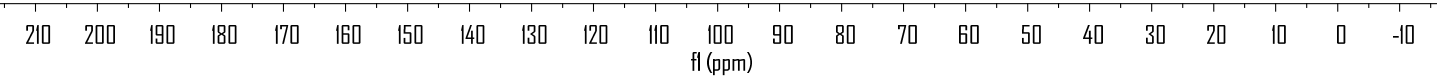
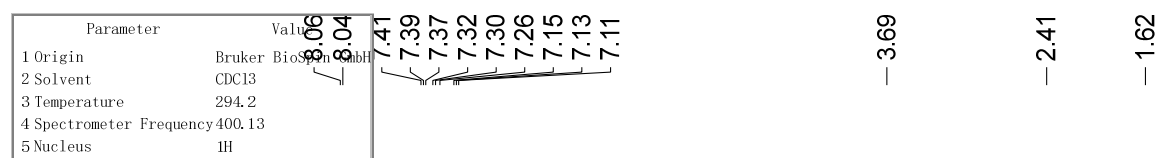

4 Spectrometer Frequency 400.

5 Nucleus $\quad 1 \mathrm{H}$<smiles>Cc1c(Br)c2ccccc2n(C)c1=O</smiles>

$4 f^{\prime}$

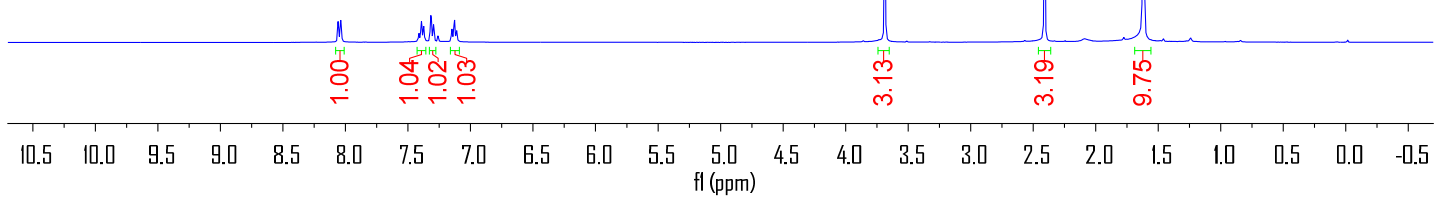



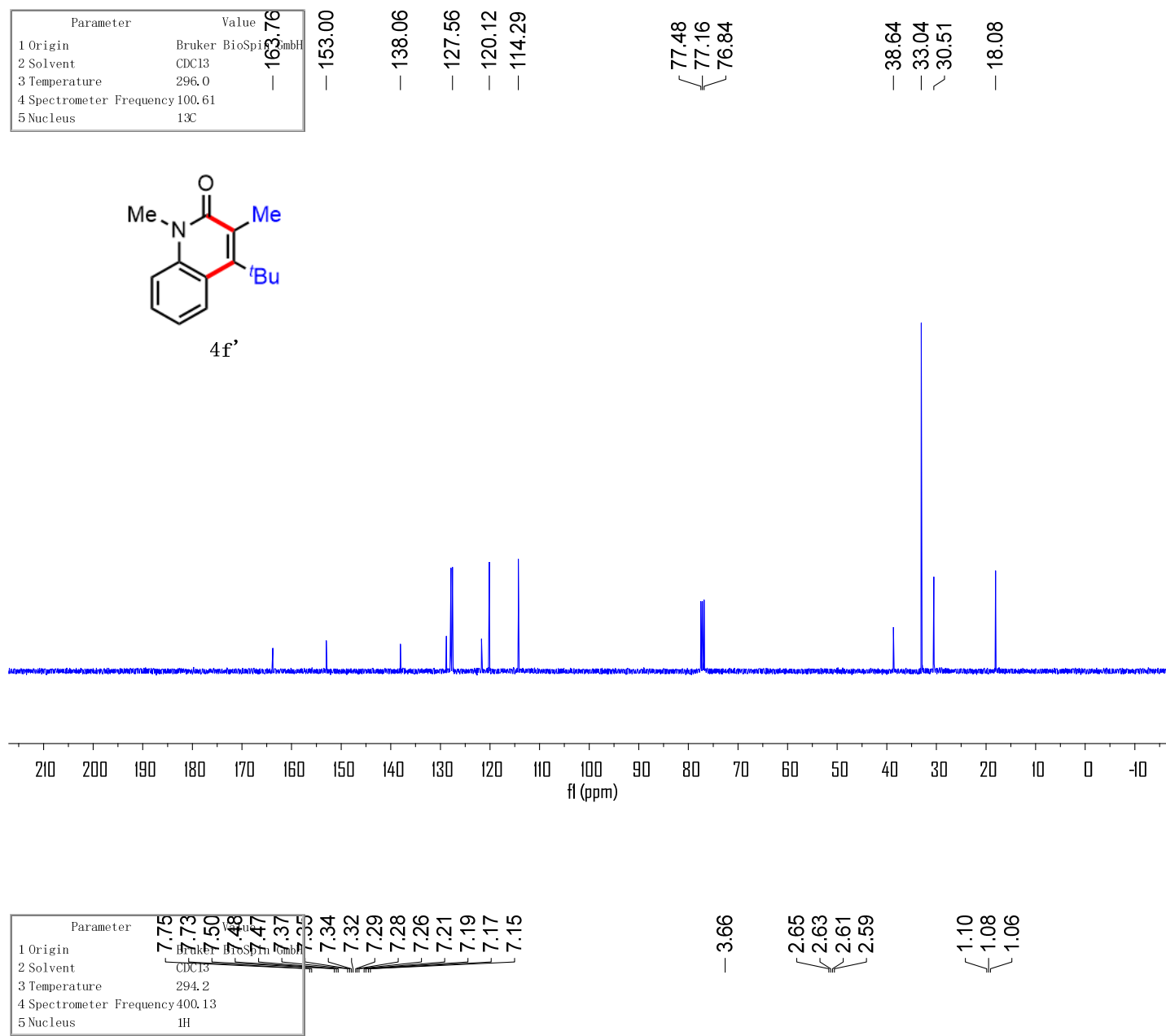

4 Spectrometer Frequency 400.13

5 Nucleus $\quad 1 \mathrm{H}$

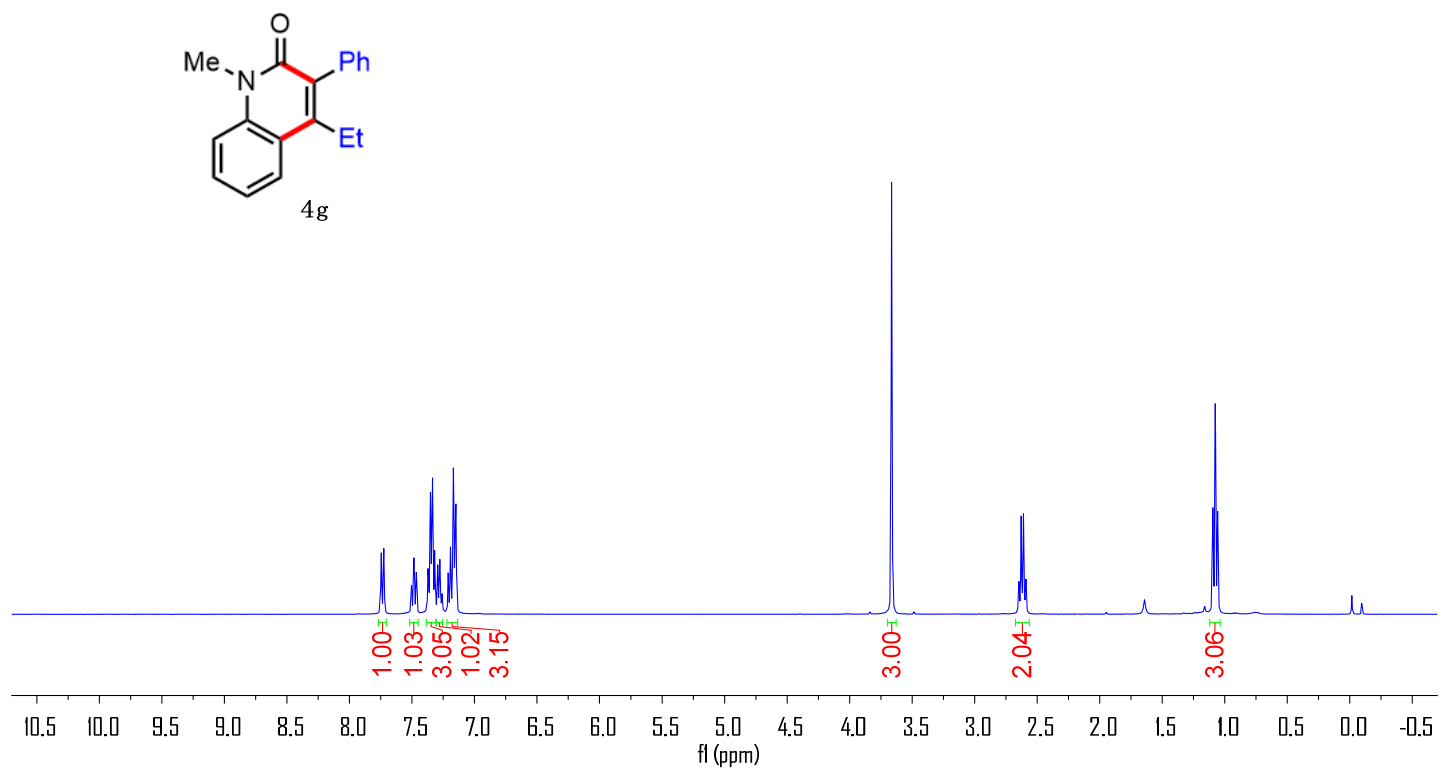




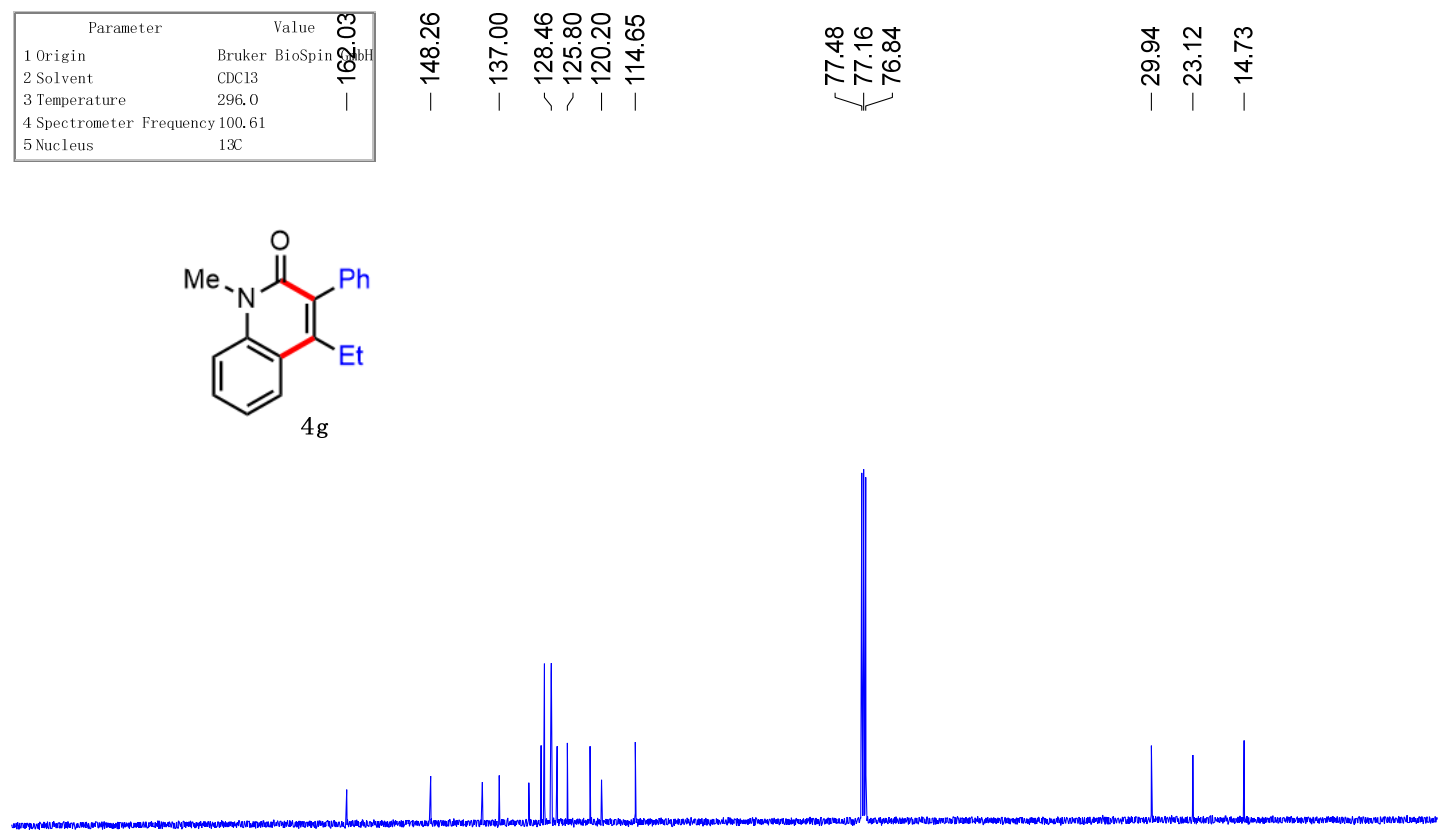

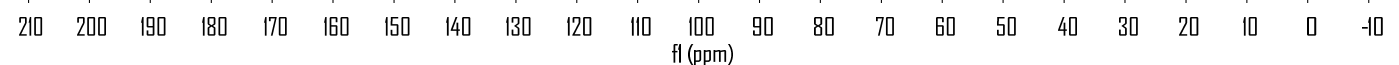

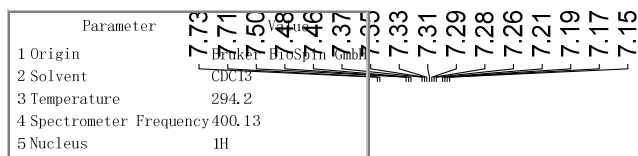

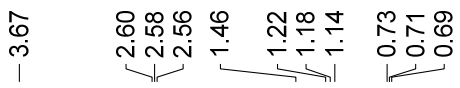<smiles>CCC(C)(C)c1c(-c2ccccc2)c(=O)n(C)c2ccccc12</smiles>

$4 \mathrm{~h}$

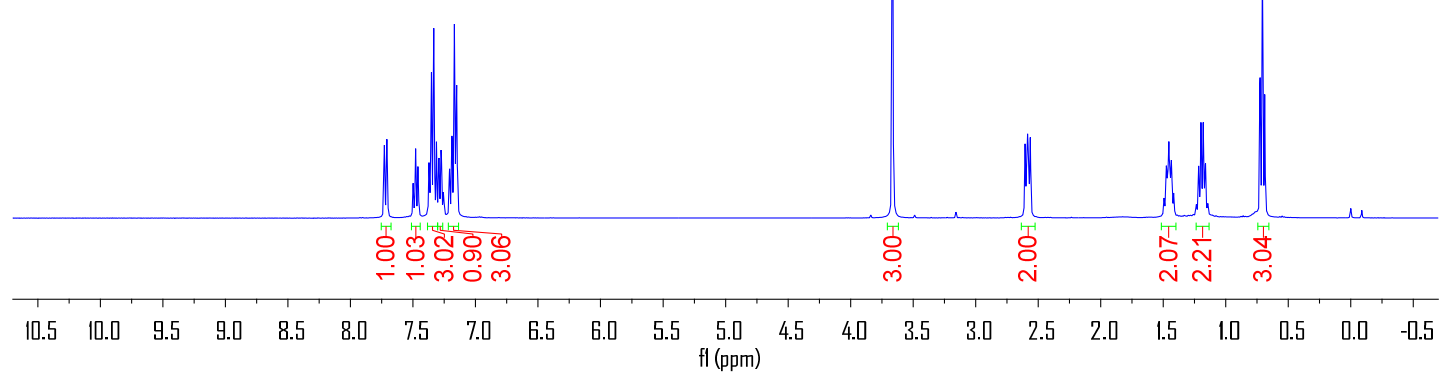




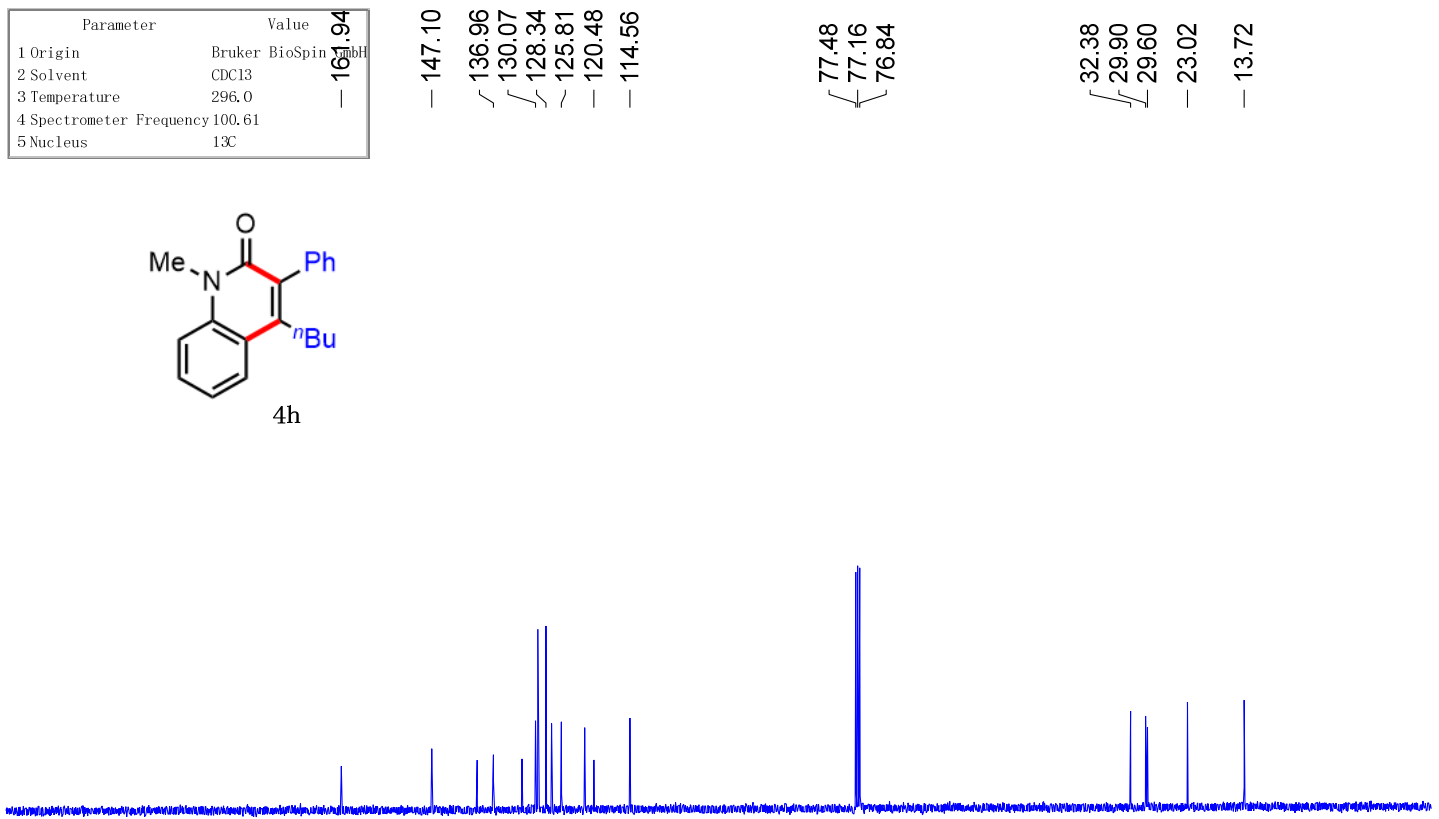

$\begin{array}{llllllllllllllllllllllllllll}210 & 200 & 190 & 180 & 170 & 160 & 150 & 140 & 130 & 120 & 110 & 100 & 50 & 80 & 70 & 60 & 50 & 40 & 30 & 20 & 10 & 0 & -10\end{array}$ 